Traditional Leafy Vegetables in Zimbabwe:

Agronomic and Market Studies

\title{
Praxedis Dube
}




\section{Thesis committee}

\section{Promotors}

Prof. Dr Wim J.M. Heijman

Special professor Regional Economics

Wageningen University \& Research

Prof. Dr Paul C. Struik

Professor of Crop Physiology

Wageningen University \& Research

\section{Co-promotor}

Dr Rico Ihle

Assistant Professor, Agricultural Economics and Rural Policy Group

Wageningen University \& Research

\section{Other members}

Dr P.G.M. Hebinck, Wageningen University \& Research

Dr P.T.M. Ingenbleek, Wageningen University \& Research

Prof. Dr U. Mazarura, University of Zimbabwe, Harare, Zimbabwe

Prof. Dr E.C. van lerland, Wageningen University \& Research

This research was conducted under the auspices of the Wageningen School of Social Sciences (WASS). 
Traditional Leafy Vegetables in Zimbabwe: Agronomic and Market Studies

\section{Praxedis Dube}

\section{Thesis}

submitted in fulfilment of the requirements for the degree of doctor

at Wageningen University

by the authority of the Rector Magnificus,

Prof. Dr A.P.J. Mol,

in the presence of the

Thesis Committee appointed by the Academic Board

to be defended in public

on Monday 17 September 2018

at 1:30 p.m. in the Aula. 
Praxedis Dube

Traditional Leafy Vegetables in Zimbabwe: Agronomic and Market Studies, 228 pages.

PhD thesis, Wageningen University, Wageningen, the Netherlands (2018)

With references, with summary in English

ISBN: 978-94-6343-500-0

DOI: $10.18174 / 457873$ 
Dedicated to my father Yarurayi Ernest (1928-1998), my mother Elivia (1932-2002) and to my sons: Zvikomborero, Tanaka \& Tendai 



\section{Contents}

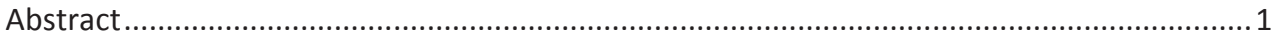

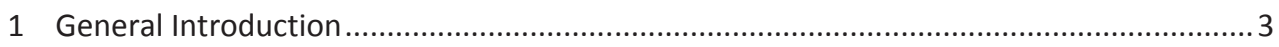

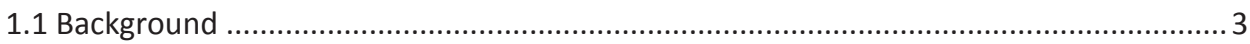

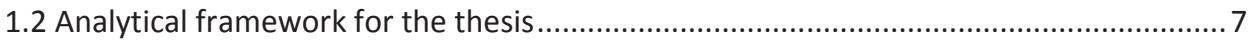

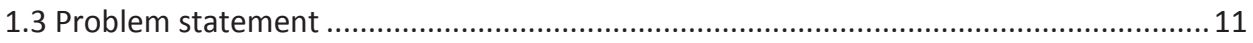

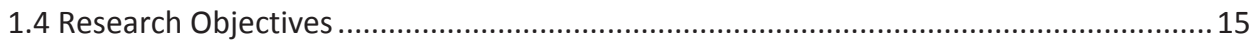

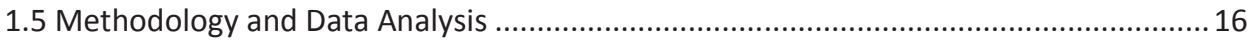

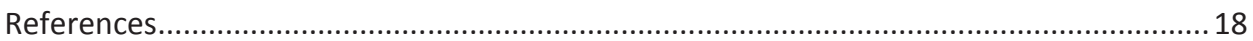

2 Seed health tests of traditional leafy vegetables and pathogenicity in plants.................... 25

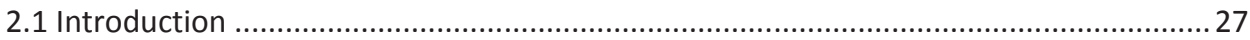

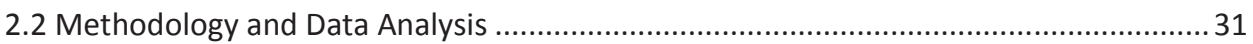

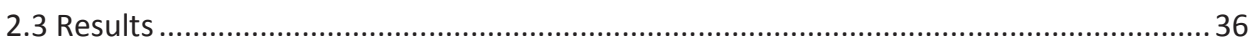

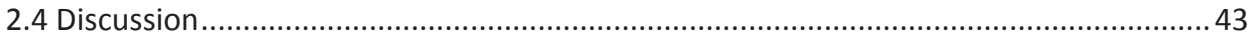

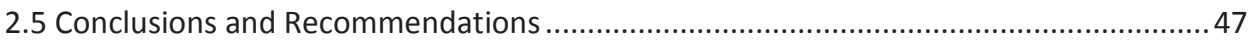

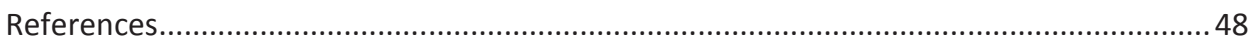

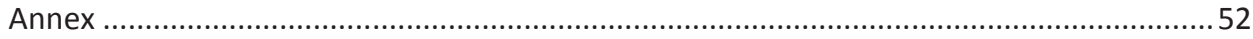

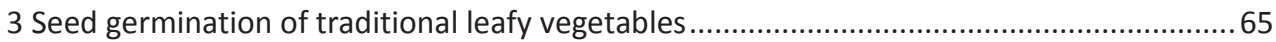

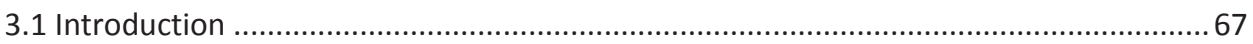

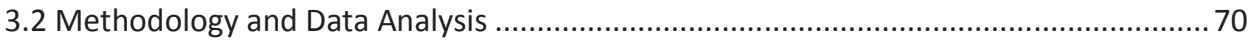

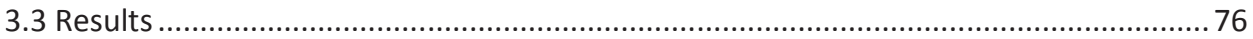

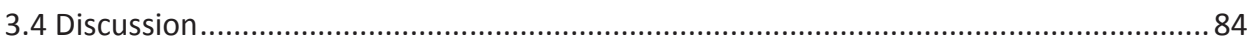

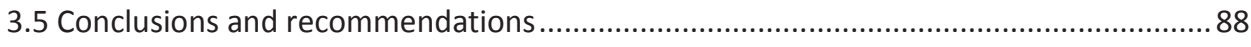

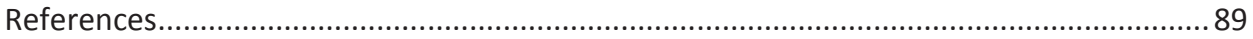

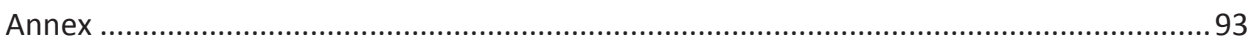

4 Determinants of household demand for traditional leafy vegetables in Zimbabwe ...........99

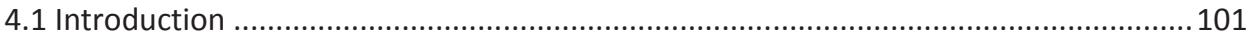

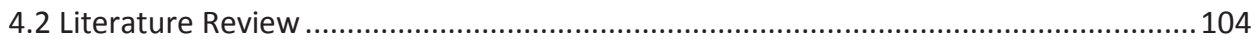

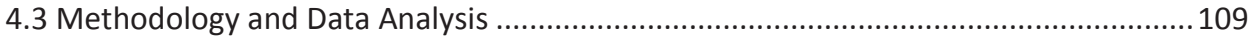

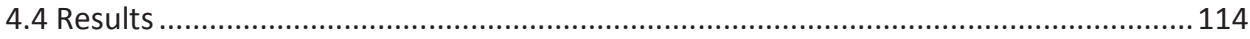

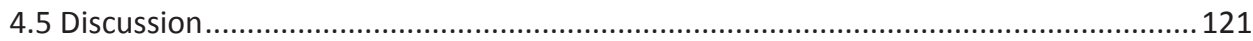


4.6 Conclusions and Recommendations..... .125

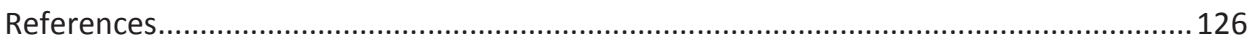

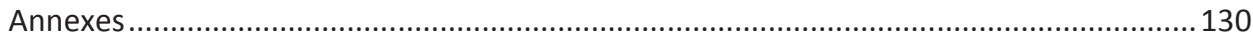

5 The potential of traditional leafy vegetables for improving food security in Africa ..........135

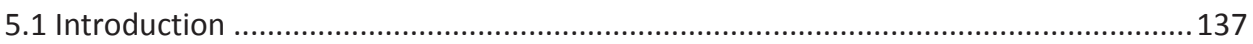

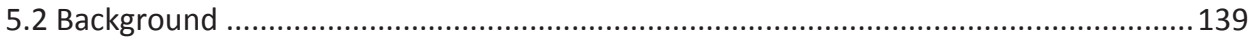

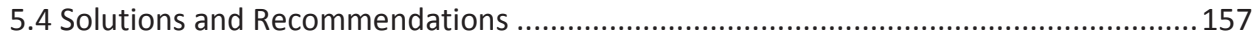

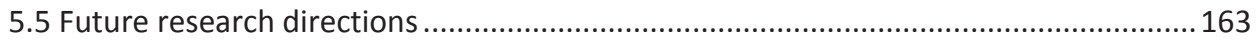

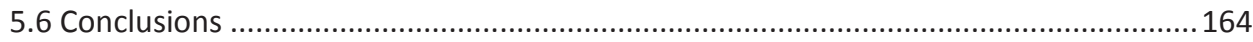

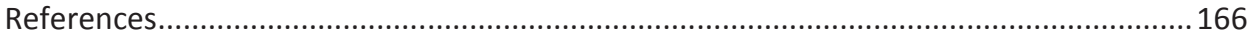

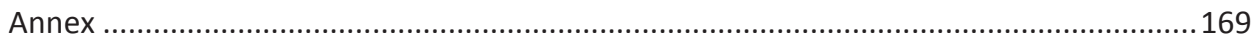

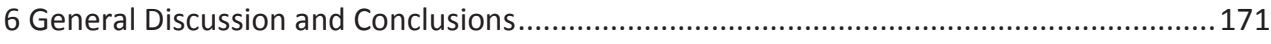

6.1 Research Objectives and Summary of Major Findings ............................................. 171

6.2 Conclusions and implications in traditional leafy vegetable supply chains.................177

6.3 Limitations and recommendations for future research ........................................... 184

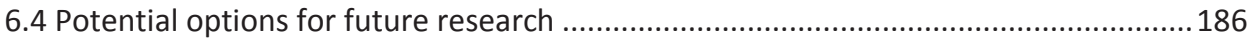

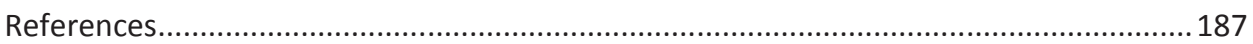

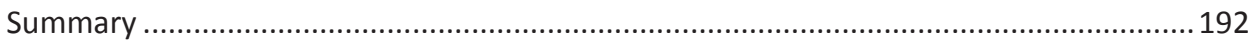

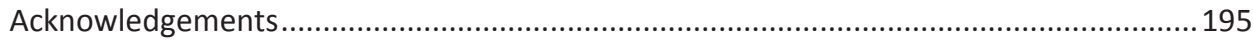

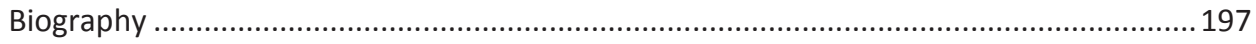

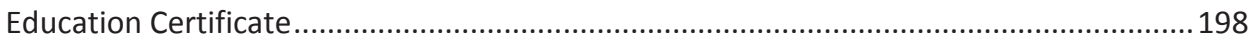

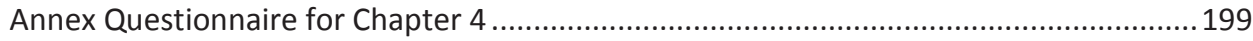




\begin{abstract}
Praxedis Dube (2018). Traditional Leafy Vegetables in Zimbabwe: Agronomic and Market Studies. PhD thesis, Wageningen University, The Netherlands, with English summary, 228 pages.
\end{abstract}

Feeding the quickly growing population in Africa remains a global challenge. As the demand for food increases, climate change, on the other hand, poses more challenges to agricultural productivity, implying a threat to the provision of sufficient quantity and quality of food. Traditional leafy vegetables in Africa are resilient to adverse weather conditions and are naturally rich in nutrients, including vitamins $A$ and $C$, iron, protein and other macro- and micronutrients. The growing importance of traditional leafy vegetables as affordable sources of micronutrients is predicated on the rising food and nutrient insecurity in many developing countries, e.g. Zimbabwe. It is in this context that the supply chain of traditional leafy vegetables was investigated. Increasing the production of traditional leafy vegetables will increase their consumption, and may also benefit the health of many people, and increase the incomes of smallholder farmers and the poor people involved in the supply chain. The study developed an analytical framework that can be used to evaluate and help researchers to come up with a general assessment of the production, markets and consumption of traditional leafy vegetables in Zimbabwe or across Sub-Saharan Africa. The framework was constructed based on the existing literature, and modified based on information gathered after assessing the traditional leafy vegetable supply chain in Zimbabwe. In addition, the study brought together seed science and economics in an interdisciplinary approach to address the problems of traditional leafy vegetables. A seed health test was conducted in order to determine the seed-borne pathogens associated with traditional leafy vegetable seeds. Seeds were collected from five different farmers in three provinces of Zimbabwe and tested for the presence of pathogens in the plant pathology laboratory. Another study was carried out with seeds from three traditional leafy vegetables sourced from five different farmers, in order to evaluate the germination of seeds and the morphological characteristics of the seedlings. The seeds were collected during the growing season of 2015-2016, stored 
at room temperature and tested for germination in the laboratory at regular monthly intervals during storage. Field germination tests were also conducted. The results from the two chapters (Chapters 2 and 3) show that the quality of the seeds depended on the seed sources. Finally, instead of only focusing on the seed science aspects, the study also took into consideration the need to assess the economic aspects of traditional leafy vegetable production and consumption. In this regard, the study examined the influence of various socio-economic characteristics of households on the quantity they consume and amount of money they spend on traditional leafy vegetables, as well as the potential of tradition leafy vegetables to improve food security. A survey was therefore conducted in four districts of Zimbabwe. The findings were subjected to two econometrics models and linear regression, and showed that consumption of traditional leafy vegetables is very low and depends on various socioeconomic characteristics of the households. The latter study involved a survey and a gross margin analysis to evaluate the potential benefits of traditional leafy vegetables across different supply chain components. Improving the productivity of traditional leafy vegetables through research will go a long way in improving their contribution to the reduction of poverty and food and nutrition insecurity. In light of the findings from each chapter, the presented supply chain framework could be organised differently. The seed regulatory services and breeding research could be embedded horizontally instead of vertically.

Keywords: seed quality, germination, seed health, determinants, socio-economic characteristics, households, econometrics models, linear regression, food and nutrition insecurity, supply chain 


\section{General Introduction}

\subsection{Background}

Malnutrition and obesity have become the greatest problems worldwide, with global estimates indicating that up to one-quarter of the world's population suffers from malnutrition (FAO, 2015) and close to 1.5 billion people are obese (WHO, 2012). The projections show that a significant rise of malnourishment in Africa will be experienced by 2050-from 223 million to 355 million-according to the Alliance for a Green Revolution in Africa. Stunting and obesity in children are increasing to 64 million and 60 million by 2020 in Africa and worldwide, respectively (de Onis et al., 2011). Micronutrient deficiencies are at alarming levels: globally, over 3 billion people suffer from micronutrient deficiencies (Gardner \& Halweil, 2000). In addition to already existing global challenges, climate change is threatening food security and the provision of adequately balanced food on a global scale, causing food gaps for certain crops and subsequently reducing food diversification. In the words of Mnzava (1997), Maundu et al. (1999), and Khoury et al. (2014), decreasing global dietary choices, decreasing diversity of traditional diets and inadequate prioritisation of balanced nutrition lie at the heart of the these global challenges.

Micronutrient deficiencies are often referred to as hidden hunger, as they can occur within the context of adequate energy intake (FAO et al., 2012) and various nutritional innovations, including the bio-fortification of staple foods. Allen et al. (2006) argue that the micronutrient deficiencies faced by poor communities in most developing countries (e.g. in Sub-Saharan Africa) can be addressed by adapting and domesticating traditional and wild micronutrientrich foods as a simple and affordable means of satisfying micronutrient needs. One of the feasible options is traditional leafy vegetables (TLVs). TLVs are cooked and consumed to accompany staple foods (e.g. maize or cassava), which are generally low in micronutrient content. TLV intake, accompanying the staples, is important for both food and nutrition security as they can help to increase the intake of staples, improve their flavour and palatability, add variety to meals and make them more healthy.

TLVs are plants which originate in Africa and have a long history of cultivation and domestication to African conditions and use (Ambrose-Oji, 2009). Natarajan (2002) listed 
about 30,000 edible plants throughout the world, of which 7,000 are grown or collected as food. Commonly consumed TLVs across Africa include amaranth (Amaranthus spp.), okra (Abelmoschus esculentus), spider flower (Cleome gynandra), pumpkin leaves (Cucurbita spp.), cowpea leaves (Vigna unguiculata), black jack (Bidens pilosa), sweet potato leaves (Ipomoea batatas), nightshade (Solanum spp.), moringa (Moringa oleifira), african eggplant (Solanum spp.), baobab leaves (Adonsonia digitata), jute (Corchorus olitorius), and cassava leaves (Manihot esculentum).

TLVs are becoming more and more important crops in many countries in Sub-Saharan Africa, due to their multiple uses; e.g. the leaves of cassava and sweet potato provide micronutrientrich vegetables, whilst their tuberous parts provide much-needed energy. TLVs combine both social and socio-economic importance across Africa as multi-purpose vegetables with both environmental adaptability and nutritional and medicinal properties (Weinberger \& Msuya, 2004; Abukutsa-Onyango, 2007; Maroyi, 2013); they also provide a source of income to poor people along the supply chain (Schippers, 2000). Traditional leafy vegetables account for around 90\% of all leafy vegetables consumed in Tanzania (Amaza, 2010), over three-quarters of the Zimbabwean population use them when available (DFID project 2309, 2003), and almost two-thirds of the rural population households in Limpopo, South Africa, consume them at least twice a day (Faber et al., 2010), underlining their importance for food and nutrition security.

Rapid urbanization, rural transformation and changes in food consumption patterns has spurred demand for agricultural produce from the smallholder farm sector, creating urbanrural linkages and expanding the markets for various crops products (Livelihoods and Food Security Programme (LFSP), 2017). Dhewa (2017) reported a shift from the consumption of modernised foods (e.g. use of maize flour in staples) to traditional foods (e.g. use of traditional crops, including TLVs) in urban areas as shown by the mushrooming of restaurants serving traditional foods. The food basket is broadening from traditional leafy vegetables, beef and sadza, bread and tea, other vegetables and a wide range of fruits (Dhewa, 2017). People are innovating around food and, as they do that, consumption is increasing, making the TLV supply chain a pertinent aspect of localised patterns of food consumption. Many Zimbabweans are now beginning to understand the fact that food is not just about staples, 
but eating from a wide range of options including small grains, TLVs and wild fruits. The rise of diseases like diabetes, cancer and HIV/AIDS have triggered increased consumption of small grains and indigenous vegetables (i.e. TLVs) (Dhewa, 2017), implying that the health of many may benefit from increased production of TLVs.

Domestication of TLVs can increase their production, and thus their consumption, which may benefit the nutritional health of many people. The production and marketing of TLVs is most associated with women; therein lies a high potential to empower women, alleviate gender vulnerability and dependence syndrome amongst women. Weinberger and Pichop (2009) estimated that the TLV market is worth billions of USD across Sub-Saharan Africa. Traditionally, TLVs are gathered in the wild, in fields or fallow lands, with few cases of cultivation across Africa: only two provinces were identified in reports of TLV cultivation in South Africa (Bvenura \& Afolayan, 2015) and only 25 species of TLVs make up the majority cultivated across Africa (Schippers, 2000). Cultivation of TLVs in home gardens would go a long way in increasing their consumption, thereby significantly reducing food insecurity and malnutrition problems (Bvenura \& Afolayan, 2015). However, some TLV species, e.g. Cleome spp., show substantial variation in reproductive characteristics relevant to domestication and crop improvement (Sogbohossou et al., 2018). Literature indicates that these TLVs are actually somewhere in the process of domestication, therefore they have a very special seed system, as some farmers grow seed, whereas most farmers collect them from wild plants or plants in fallow fields. Farmers collect and save seeds, and exchange them with or sell them to other farms. Mostly healthy plants are protected in the field to produce seeds (Tata et al., 2016), whilst breeders identify traits, i.e. leaf size, taste, resistance to disease, etc. and incorporate these traits using classical breeding and molecular techniques (Tata et al., 2016).

TLV seeds are hardly considered in Zimbabwe's seed policies as they are not covered in many seed regulatory platforms (Dube \& Mujaju, 2013), giving them less economic value in local, regional and international markets. Because of the omission of TLV seeds in local seed policies, both the public and private sectors do not give enough attention or invest much effort in the production, distribution or development of quality standards for these seeds, leaving farmers with no option but to use their saved seeds of unknown quality. Poor seeds give low germination, thereby thus farmers fail to meet their desired plant population per 
area, and end up using more seeds than required, thereby increasing their production costs. Stringent phytosanitary rules and seed regulations across countries impede the movement of TLV seeds, thereby limiting farmers' access to new varieties of seeds which might be better yielders, directly affecting their incomes. One way of addressing the problems of TLVs in Zimbabwe is by bringing the two disciplinary domains of seed science and economics together in an interdisciplinary way, as depicted by the analytical framework discussed below.

The analytical framework presented in this study was developed after an extensive literature review and the surveys that constitute Chapters 4 and 5 . The supply chain consists of quite a number of components forming an economic entity, which distributes benefits and apportion risks among participants. 


\subsection{Analytical framework for the thesis}

Figure 1 shows the analytical framework for TLVs: complete supply chain of TLVs from production to consumption in Zimbabwe and the aspects focused on in this thesis. It also shows aspects of the chain that are the focus of this thesis. The framework creates a new data structure which is helpful for summarizing the data in a way which supports answering the main research question: How is the TLV supply chain organized in Zimbabwe?

The components of the TLV supply chain are shown in Figure 1. The numbers refer to the chapters $(2,3,4$ and 5$)$ that deal with the component indicated.

Component 'seed farmers' $(2,3$, and 5 ): Seed quality aspects, a mandate carried by the Department of Regulatory Services (DARS) and survey research for Chapter 5 . The benefit of seed testing include: farmers come up with desired plant population; farmers get more income; spread of diseases is reduced; yields increase; quality of the vegetables on the markets increases. For example, TLVs have the potential to contribute to food security and economic development in many developing countries (Schippers, 2000), including Zimbabwe.

Component 'local consumers' (4, 5 ): Household consumption of and expenditure for TLVs, and insights from Chapter 4 are used in Chapter 5 . The fourth chapter focuses on consumers, examining the determinants of household consumption and expenditure for TLVs.

Chapter 5 deals with a number of components (See Figure 1). It is based on results obtained from the surveys conducted among seed and vegetable growers, traders, processors and marketers as depicted in Figure 1, and insights from the literature review and data analysis in Chapter 4. The gross margin calculations have been based on farmers' perception of the costs required for production, which can be a realistic way of convincing them to use high quality seeds, or improved varieties they can achieve better income returns from their produce. 
Figure 1 Analytical framework of the thesis: TLV supply chain in Zimbabwe and the studied areas
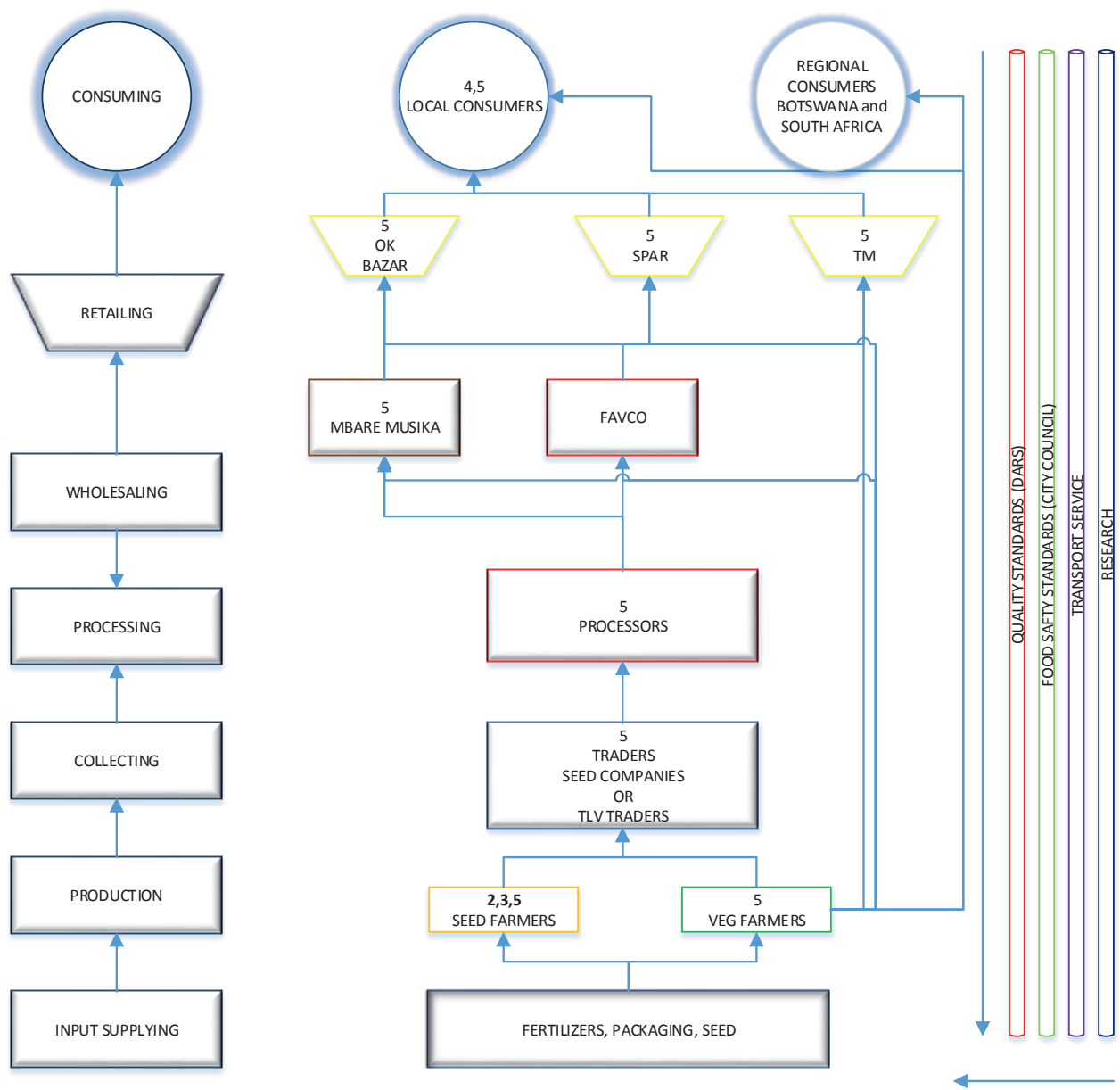

Source: Author based on Benson et al. (2013) and Djalalou-Dine Ademonla (2013).

TLV seeds are traditionally traded through informal seed systems (Croft et al., 2017) where seed quality is not known, and is typically poor (Abukutsa-Onyango, 2007). Informal seed systems have been described by Almekinders (2000) as the total seed production activities of farmers, mostly small-scale farmers. The advantages of the informal seed systems include: farmers have control over seeds; timely availability of seeds; relatively cheaper seeds (Almekinders, 2000)- a key component of supporting domestic marketing structures in 
developing countries. The disadvantages of the informal seed systems include: low germination, farmers are forced to use large quantities of seed to compensate for poor germination rates, resulting in increased production costs and low yields.

Although the vertical components of the analytical framework shows the quality control component, the literature shows that TLV seeds rarely undergo any quality control before being shared, exchanged or marketed amongst the farmers. There are no formal quality standards for seeds, not even formal procedures to test seed quality. There has been no breeding, and therefore there is little resistance against pests and diseases, as illustrated by the fact that the seeds are infected by all kinds of cosmopolitan and opportunistic diseases (Ma et al., 2013). Due to lack of genetic improvement, TLV crops produce inferior yield in terms of both quality and quantity (Tadele, 2009). The yields are very low, resulting in TLV farmers failing to meet the demand and deliver quality produce to consumers (Afari-Sefa et al., 2013). The objective of this study, using the components numbered 2 and 3 (referring to the respective chapters of the thesis), is to determine the quality of farmers' saved seeds through conducting seed health and germination tests.

TLVs are commonly eaten and regarded as valuable crops in rural areas of Africa as important cheap sources of food and nutrition (Schippers, 2000; Bvenura \& Afolayan, 2015) and income generation for resource-poor farmers (Tadele, 2009). Both poverty and food insecurity are major challenges for people in rural Africa. TLVs have been historically regarded as food for the poor, because their prices are relatively affordable compared to other food (Schippers, 2000). TLV production is affordable and can be realized by poor people, requiring only small capital investment (Schippers, 2000). This study assesses the role of TLVs in smallholder farmers' diets and livelihoods using the components marked 5 (Chapter 5 of the thesis).

With emphasis on knowledge sharing, on the importance and benefits associated with TLV consumption, availability of TLVs in modern markets, i.e. supermarkets, TLVs are no longer typically regarded only as food for the poor, and literature reports that their consumption has been realised in urban areas (e.g. Schippers, 2000). However, in Sub-Saharan Africa, per capita consumption of vegetables is still below recommended levels (Ruel et al., 2005). 
Several challenges impede the consumption of vegetables across Sub-Saharan Africa, including household income levels, preferences by households, market prices and availability, and the gender of the decision maker in the household (Ruel et al., 2005). Elsewhere in the world, socio-demographics (including education, age, ethnicity, income and location) were identified as predictors of vegetable consumption in Malaysia (Yen et al., 2011).

Weinberger \& Msuya (2004) reported an inverse relationship between household income and wealth and TLV consumption. Traditionally, poor people regularly consume TLVs, implying that they are probably regarded as food of inferior quality (Jansen van Rensberg et al., 2007). In addition, the elite urban dwellers and middle-income earners consume less TLVs, for fear of risking their health (World Vegetable Center, 2006). Preferences may vary per region. For instance, spider flower is the most cultivated and preferred TLV species in both Zimbabwe and Southern Africa (Bvenura \& Afolayan, 2015). Consumers in western Kenya value TLVs for their taste, nutritional and medicinal benefits (Abukutsa-Onyango, 2007). The objective of Chapter 4 of the thesis is to examine the determinants of TLV consumption in Zimbabwe and uses the local consumer component of the framework for this research. 


\subsection{Problem statement}

Chweya \& Eyzaguirre (1999) and Pasquini et al. (2014) reported that there is very little published information on the supply chain of TLVs. Formal market chains and quality standards for this supply chain barely exist (Smith \& Eyzaguirre, 2007; Pasquini et al., 2014). The TLV markets have been relatively ignored; very little literature has been published on the supply chain of TLVs, with a few exceptions: e.g. Chagomoka et al. (2014), who analyzed the value chain of TLVs in Malawi and Mozambique. They found that linkages between value chain actors were weak. Abukutsa-Onyango (2007) pointed out the importance of farmers having access to high quality seed from the start, as a way to improve TLV productivity and supply. Similarly, Smith \& Eyzaguirre (2007) emphasised the urgent need for research that addresses agronomic constraints, including seed quality and diseases. The present thesis argues that if consumption increases with limited supply, the potential of TLVs for food and nutrition can hardly be realized.

Although TLVs have been an integral part of agricultural systems in Africa, most countries have not given them priority in crop development. It has been reported that over 350,000 plant species are available in the world, 80,000 plant species are edible to humans and only 150 are actively cultivated for consumption (Füleky, 2002). TLVs, on the other hand, do not feature significantly on the research agenda, for example, in the fields of breeding and agronomy, including the use of irrigation systems to improve productivity and year-round production to eliminate seasonality. Studies have shown that retailers and consumers regard taste, appearance, nutritional value, long shelf life, freshness and affordability as important attributes that could be targeted by breeders to increase TLV consumption (Afari-Sefa et al., 2012). Typically, smallholder farmers seek TLV varieties with rapid germination and no period of dormancy, multiple leafy harvests, high yields and resistance to diseases and pests, among other important characteristics. In view of the global food and nutrition demands, and poverty and gender inequality enshrined in the Sustainable Development Goals, TLVs already under cultivation paint a gloomy picture of improving world food and nutritional needs and meeting ever increasing food and nutritional demands, poverty reduction and addressing gender inequality. Studies have shown that people consume large quantities of fresh TLVs 
compared to dried TLVs (Banwat et al., 2012). The regular supply of fresh TLVs can be enhanced through irrigation systems.

TLV production in many Sub-Saharan African countries faces many challenges, including availability of quality seeds and poorly skilled farmers, which contribute to low productivity and seasonality of supply, in turn affecting their consumption. Because of the limited agronomic knowledge of smallholder farmers, particularly regarding the agronomic value of high-quality seeds (Biemond et al., 2012), lack of information on seed storage and difficulties with seed germination (Cernansky, 2015), these farmers hardly get one-tenth of the potential yield from seeds (Ojiewo et al., 2010). Nevertheless, the seeds used are rarely tested for germination and pathogens before planting, risking undesirable plant populations or using more seed than required. The risks are high also for seed degeneration across generations. Taken together, all these challenges contribute to sub-optimal and decreased yields and profits which may subsequently lead to the less successful commercialization of TLVs. As a result, farmers fail to meet the growing demand (Afari-Sefa et al., 2013) and availability is also limited to certain periods of the year. Farmers may also risk the quality of their vegetables (Ayana et al., 2014) due to diseases, as some plant diseases may affect the plants during the growing phase as well as in storage (Ngadze, 2014). Also, the lack of documentation on preferred species (Maundu et al., 1999), lack of seed selection for uniformity of desired traits (Smith \& Eyzaguirre, 2007), and poorly developed seed supply and distribution (Ayana et al., 2014) hinder TLV production and consumption.

Despite their potential to address current global challenges including food and nutrition insecurity caused by climate change and malnutrition, daily TLV consumption varies across Sub-Saharan Africa, ranging from $24 \mathrm{~g}$ (in Senegal) to $350 \mathrm{~g}$ (in Tanzania) per capita, as depicted by Weinberger \& Pichop's ( $20 \emptyset$ f factors, such as the seasonality of supply and prices of TLVs and the socio-economic characteristics of households. The seasonality of TLVs affects their pricing; prices fall in the rainy season because they grow well during that period (Schippers, 2000). Moreover, most rural households hardly buy TLVs from the markets, as they collect them from fields and forests (Weinberger \& Msuya, 2004). Inadequate supply due to production constraints (e.g. use of poor-quality seeds which contributes to poor crop stands, reduced yields, and 
subsequently, low supply of vegetables), differences in socio-demographic, socio-economic and other consumer characteristics (Weinberger \& Msuya, 2004; Abukutsa-Onyango, 2007; Odhav et al., 2007; Karanja et al., 2010; Banwat et al., 2012) also affect TLV consumption.

This thesis is motivated by the fact that, on one hand, quite a volume of research has been done on consumption of TLVs, while, on the other hand, little research has been done on production, particularly on seed quality and seed markets, to help supply meet demand. It is against this background that the present thesis, which combines technical and socioeconomic methods, assesses the supply chain of traditional leafy vegetables. On one side, the thesis evaluates the potential of traditional leafy vegetables for improving food security and nutrition. It also explores the determinants of household consumption and expenditure for traditional leafy vegetables using surveys. Several determinants impede the consumption of TLVs, which may play a significant role in overcoming the dietary deficiencies experienced across Sub-Saharan Africa. If such impediments are exposed and recommendations are made for future research, subsequent developments may lead to increased consumption of TLVs, which may benefit the health of many, and improve the incomes of those involved in the supply chain. On the other side, the thesis examines the quality of TLV seed by conducting germination and seed health tests before it is sown. If the farmers sell their seeds with known germination capacity or quality, this may substantially increase their incomes, as their seeds will more likely fetch higher prices. Research on and documentation of seed quality is of equally great importance, so that production can be increased to meet urgent demands, and seeds can be conserved and used sustainably to reduce the extinction of certain species through overharvesting. This thesis shifts attention from studying one segment of the supply chain and looks into operations within different segments. No such literature has been reported. Such a synthesis is expected to benefit almost the entire supply chain (the supply side, i.e. producers and marketers, and the demand side, i.e. the consumer). This makes this thesis novel.

This thesis focuses on the supply chain of traditional leafy vegetables using an interdisciplinary approach and uses Zimbabwe as an example. Zimbabwe was selected for several reasons: Zimbabwe imported goods valued at 2 billion USD in the first quarter of 2016, with vegetables and citrus fruits accounting for over 15 million USD (Kachembere, 2016), despite the fact that 
Zimbabwe is a low-income country. However, the consumption of vegetables is still very low. Earlier reports have shown a daily intake of $30 \mathrm{~g}$ per capita (FAO, 2015), which has resulted in obesity and malnutrition in the population. Over one-third of 15 - to 49 -year-old women, $12 \%$ of men and $6 \%$ of children under age five are overweight or obese. On the other hand, onethird of children under age five are stunted. Currently, in Zimbabwe anemia affects about 37\% children under five, about one quarter of women and 15\% of men between 15 and 49 years of age (Zimbabwe National Statistics Agency and ICF International 2015, 2016). Anemia is a disease related to low intake of iron, which can be found in great abundance in some TLVs, e.g. Amaranthus spp. and cowpea. In recognition of these national health challenges, the Zimbabwean government introduced the Statutory Instrument 120 of 2017, Mandatory Food Fortification Programme, aimed at compelling all food processing companies to add nutrients to everyday foods such as bread, mealie meal, cooking oil and sugar to enrich consumers' diets (Chikwati, 2017). But the type of products purchased may depend on the household income, with low income households (constituting over 70\% of Zimbabwean population) tending to consume bread, sugar, cooking oil and pre-processed mealie meal less frequently, posing a considerable challenge to addressing domestic health problems in this manner. Considering the coexistence of malnutrition and obesity in Zimbabwe, increasing the intake of vegetables, particularly TLVs, is therefore becoming an important initiative. As these challenges are widespread across other Sub-Saharan countries as well, I believe that this thesis contains an insightful analysis relevant for Sub-Saharan Africa in general, and findings can be used to tackle similar problems in the region. 


\subsection{Research Objectives}

I am interested in the following specific research objectives:

0 1: Detecting and identifying seed-borne pathogens on TLV seed and establishing the relationship between seed infection and pathogens which develop on the plants in the field (Chapter 2);

O 2: Evaluating the germination of traditional leafy vegetable seed and determining the morphological characteristics of seed from five sources (Chapter 3);

$O$ 3: Examining the determinants of household consumption and expenditure for TLVs (Chapter 4);

O 4: Assessing the potential of traditional leafy vegetables for improving food security in Africa (Chapter 5). 


\subsection{Methodology and Data Analysis}

To address the research objectives listed above, four researches were conducted: two researches on the quality of TLV seed-germination tests and seed health tests-and two surveys assessing the role played by TLVs in smallholder farmers' diets and incomes, and determinants of household consumption of and expenditure for TLVs. Three species, namely Cleome gynandra, Bidens pilosa and Amaranthus hybridus, were evaluated for quality and used as examples. In our study, the term quality refers to physiological characteristics, including the percentage purity and germination, the uniformity of the plants and seed health (i.e. free of seed-borne pathogens). Each chapter is written as a stand-alone publication for a specific scientific journal. The methodology used for each chapter is presented as follows.

Table 1 shows a summary of the research methods used for each chapter, where each objective is addressed in a separate chapter.

Table 1. Summarised Research Methodology

\begin{tabular}{|c|c|c|c|c|}
\hline $\begin{array}{l}\text { Chapter/ } \\
\text { Objective }\end{array}$ & Focus & Data collection & Level of analysis & Data analysis \\
\hline $\begin{array}{l}\text { Chapter } 3 \\
\text { O } 2\end{array}$ & Seed germination & Laboratory test & $\begin{array}{l}\text { Analysis of variance (ANOVA) in } \\
\text { GenStat }\end{array}$ & GenStat \\
\hline $\begin{array}{l}\text { Chapter } 4 \\
\text { O } 3\end{array}$ & $\begin{array}{l}\text { Household consumption } \\
\text { and expenditure }\end{array}$ & Survey & $\begin{array}{l}\text { Econometric equations and } \\
\text { multivariate linear regression } \\
\text { models }\end{array}$ & SPSS \\
\hline
\end{tabular}

Source: Author

Chapter 2 is about the detection and identification of seed-borne pathogens on TLV seeds and establishes the relationship between seed infections and pathogens which develop on plants in the field; it addresses research objective 1. The presence of pathogens on seeds and plant parts was detected in the plant pathology laboratory of the University of Zimbabwe. The pathogenicity tests were carried out to confirm the presence of the pathogens on the plants 
but were not scored or scaled to show the severity of infection. Both the type of pathogens and pathogenicity test results were reported.

Chapter 3 addresses research objective 2 and evaluates the germination of traditional leafy vegetable seeds and determining the morphological characteristics of seeds from five sources. Laboratory and field germination tests were conducted and reported here. Qualitative and quantitative morphological analyses were conducted and reported various characteristics for both seedlings and 1000 seeds weight.

Chapter 4 addresses the third research objective and evaluates the determinants of household consumption and expenditure for TLVs in four districts of Zimbabwe. This chapter assesses the relationship between the quantity of TLVs consumed and amount of money spent on TLVs, as affected by various socio-economic, socio-demographic of households. Therefore, various socio-economic, socio-demographic of households affecting quantity and expenditure were evaluated.

Chapter 5 evaluates the potential of traditional leafy vegetables for improving food security in Africa, answering the last research objective. The role of TLVs in the supply chain (from production to consumption) of Zimbabwe were assessed. The content of this chapter is partly based on the results obtained from this survey, insights from the literature and insights from the data analysis of chapter 4.

Chapter 6 provides a synthesis of the main results found in all the other chapters. 


\section{References}

Abukutsa-Onyango, M. O. (2007). Seed production and support systems for African leaf vegetables in three communities in western Kenya. African Journal of Food Agriculture and Nutrition Development. 7(3): 1-16.

Afari-Sefa, V., Chagomoka, T., Karanja, D. K., Njeru, E., Samali, S., Katunzi, A., Mtwaenzi, H., \& Kimenye, L. (2013). Private contracting versus community seed production systems: Experiences from farmer-led seed enterprise development of indigenous vegetables in Tanzania. Acta Horticulturae. 1007 (1): 671-680.

Afari-Sefa, V., Tenkouano, A., Ojiewo, C., Keatinge, J. D. H., \& Hughes, J. d'A. (2 01212 ). Vegetables breeding in Africa: Constraints, complexity and contributions toward achieving food and nutritional security. Food security: The science, sociology and economics of food production and access to food. 4 (1): 115-127.

Allen, L., de Benoist, B., Dary, O., \& Hurrell, R. (Eds.) (2006). Guidelines on food fortification with micronutrients. Washington, DC: World Health Organization; Food and Agriculture Organization of the United Nations.

Almekinders, C. (2000). The importance of informal seed sector and its relation with the legislative framework. Paper presented at GTZ, Eschborn. Technology and Agrarian Development, Wageningen University, Wageningen. 1-16.

Amaza, P. S. (2010). An analysis of Traditional African vegetables and sweet potato consumer demand in Kenya and Tanzania. Technical Report submitted to AVRDC - The World Vegetable Center, Arusha, Tanzania.

Ambrose-Oji, B. (2009). Urban food systems and African indigenous vegetables: Defining the spaces and places for African indigenous vegetables in urban and peri-urban agriculture. In C. M. Shackleton, M. W. Pasquini, \& A. W. Drescher (Eds.), African Indigenous Vegetables in Urban Agriculture (pp. 1-33). London: Earthscan.

Ayana, A., Afari-Sefa, V., Emana, B., Dinssa, F. F., Balemi, T., \& Temesgen, M. (2014) Analysis of vegetable seed systems and implications for vegetable development in the humid tropics of Ethiopia. International Journal of Agriculture and Forestry. 4 (4): 325-337.

Banwat, M. E., Lar, L. A., Daber, J., Audos, S., \& Lassa, S. (2012). Knowledge and intake of fruit and vegetables consumption among adult in urban community in north central Nigeria. Nigeria Health Journal. 12 (1): 12-15. 
Benson, T., Spielman, D., \& Kasa, L. (2013). Direct seed marketing program in Ethiopia: An operational evaluation to guide seed-sector reform. Washington, DC: International Food Policy Research Institute.

Berkum, S. van, Achterbosch, T. J., \& Linderhof, V. G. M. (2017). Dynamics of food systems in Sub-Saharan Africa: Implications for consumption patterns and farmers' position in food supply chains. Report 2017-072. Wageningen: Wageningen Economic Research.

Biemond, P. C., Stomph, T. J., Kamara, A., Abdoulaye, T., Herane, S., \& Struik, P. C. (2012). Are investments in an informal seed system for cowpeas a worthwhile endeavour? International Journal of Plant Production. 6 (3): 367-386.

Bvenura, C., \& Afolayan, A. J. (2015). The role of wild vegetables in household food security in South Africa: A review. Food Research International. 76: 1001-1011.

Cernansky, R. (2015). Super vegetables long overlooked in parts of Africa, indigenous greens are now capturing attention for their nutritional and environmental benefits. Nature. 522: 146-148.

Chagomoka, T., Afari-Sefa, V., \& Pitoro, R. (2014). Value chain analysis of traditional vegetables from Malawi and Mozambique. International Food and Agribusiness Management Review. 17 (4): 59-86.

Chigumira, F. (1998). Conservation and use of Indigenous vegetables in Zimbabwe. Paper presented to the Arusha conference (unpublished).

Chweya, J. A., \& Eyzaguirre, P. B. (1999). Introduction to J. A. Chweya \& P. B. Eyzaguirre (Eds.) Biodiversity of traditional leafy vegetables. Rome: International Plant Genetic Resources Institute.

Croft, M. M., Marshall, M. I., \& Weller, S. C. ( $\left.\begin{array}{lllll}2 & 0 & 1 & 4\end{array}\right)$. Consumers' preference for quality in three African indigenous vegetables in Western Kenya. Journal of Agricultural Economics and Development. 3 (5): 67-77.

Croft, M. M., Marshall, M. I., Odendo, M., Ndiya, C., Ondego, N. N., Obura, P., \& Hallett, S. G. (2017). Formal and informal seed systems in Kenya: Supporting indigenous vegetable seed quality. Journal of Development Studies. 54 (4): 758-775.

De Groote, H., Kimenju, S. C., \& Morawetz, U. B. (2011). Estimating consumer willingness to pay for food quality with experimental auctions: The case of yellow versus fortified maize meal in Kenya. Agricultural Economics. 42: 1-16. 
de Onis, M., Blössner, M., \& Borghi, E. (2011). Prevalence and trends of stunting among preschool children, 1990-2020. Public Health Nutrition. 15 (1): 142-148.

Dhewa, C. (2017). How agric markets signal changes in consumption patterns. Herald, 24 August 2017. https://www.herald.co.zw/how-agric-markets-signal-changes-inconsumption-patterns/.

Dhewa, C. (2017). Changes in Consumption Patterns final draft August 2017. Vimeo video, posted by "Reelnet Multimedia." $122: 32$ min. Last accessed 2 August 2018 . https://vimeo.com/230421189.

DFID Research4Development Project Record (2003). Improving the livelihoods of peri-urban vegetable growers through markets promotion of fresh and processed indigenous vegetables. https://assets.publishing.service.gov.uk/media/R7487_FTR.pdf.

Djalalou-Dine Ademonla, A. A. (2013). Governance, marketing and innovations in Beninese pineapple supply chains: A survey of smallholder farmers in South Benin. PhD diss. http://edepot.wur.nl/254244.

Faber, M., Oelofse, A., Van Jaarsveld, P. J., Wenhold, F. A. M., \& Jansen van Rensburg, W. S. (2010). African leafy vegetables consumed by households in the Limpopo and KwaZulu-Natal provinces in South Africa. South Africa Journal of Clinical Nutrition. 23 (1): 30-38.

Food and Agriculture Organization of the United Nations (FAO) (2010). Special Report FAO/WFP Crop and Food Security Assessment Mission to Zimbabwe. 9 August. Rome: Food and Agriculture Organization of the United Nations; World Food Programme.

Food and Agriculture Organization of the United Nations (FAO) (2015). The state of food insecurity in the world 2015: Meeting the 2015 international hunger targets; Taking stock of uneven progress. Rome: Food and Agriculture Organization of the United Nations.

FAO, WFP \& IFAD (2012). The state of food insecurity in the world 2012: Economic growth is necessary but not sufficient to accelerate reduction of hunger and malnutrition. Rome: Food and Agriculture Organization of the United Nations.

Fuller, J. B., O'Conor, J., \& Rawlinson, R. (1993). Tailored logistics: The next advantage. Harvard Business Review. 71 (3): 87-98. 
Gido, E. O., Ayuya, O. I., Owuor, G., \& Bokelmann, W. (2017). Consumer acceptance of leafy African indigenous vegetables: Comparison between rural and urban dwellers. International Journal of Vegetable Science. 23 (4): 346-361.

Gurmu, F., Hussein, S., \& Laing, M. (2014). The potential of orange-fleshed sweet potato to prevent vitamin A deficiency in Africa. International Journal for Vitamin and Nutrition Research. 84 (1-2): 65-78.

Irungu, C., Mburu, J., Maundu, P., Grum, M., \& Hoeschle-Zeledon, I. (2011). The effect of market development on-farm conservation of diversity of African leafy vegetables around Nairobi. International Journal of Humanities and Social Science. 8 (1): 198-207.

Kachembere, J. (2016). Zim bans basic commodities imports. Daily News, 20 June 2016. https://www.dailynews.co.zw/articles/2016/06/20/zim-bans-basic-commoditiesimports.

Karanja, D., Okoko, N., Kiptarus, E., Okongo, P., Samali, S., Katunzi, A., Mtwaenzi, H., Mwakitwang, F., Afari-Sefa, V., Musebe, R., Kimani, M., \& Kimenye, L. (2010). Promoting farmer-led seed enterprises of African indigenous vegetables to boost household incomes and nutrition in Kenya and Tanzania. Paper presented at the Conference on Agricultural Biodiversity in Africa. Proceedings, pp. 1-13.

Keatinge, J. D. H., Hughes, J. d'A., Tenkouano, A., Hamilton, K., Easdown, W. J., \& Mongi, H. O. (2009). Vegetables and small private-sector interests. In A. G. Brown (Ed.), World Food Security: Can private sector $R \& D$ feed the poor? (pp. 60-68). Proceedings, Crawford Fund for International Agricultural Research, Canberra, 27-28 October.

Keding, G. B., Weinberger, K., Swai, I., \& Mndiga, H. (2007). Diversity, traits and use of traditional vegetables in Tanzania. Technical Bulletin No. 40. Shanhua: World Vegetable Center.

Khoury, C. K., Bjorkman, A. D., Dempewolf, H., Ramirez-Villegas, J., Guarino, L., Jarvis, A., Rieseberg, L. H., \& Struik, P.C. (2014). Increasing homogeneity in global food supplies and the implications for food security. Sustainability Science Agricultural Sciences. 111 (11): 4001-4006.

Livelihoods and Food Security Programme (LFSP). Changes in Consumption Patterns final draft August 2017. https://vimeo.com/230421189. 
Louwaars, N. P., \& De Boef, W. S. (2012). Integrated seed sector development in Africa: A conceptual framework for creating coherence between practices, programs, and policies. Journal of Crop Improvement. 26: 39-59.

Luckmann, J., Ihle, R., Kleinwechter, U., \& Grethe, H. (2014). World market integration of Vietnamese rice markets during the 2008 food price crisis: Food security. The Science, Sociology and Economics of Food Production and Access to Food. 6 (6): 1-17.

Ma, L., Geiser, D. M., Proctor, R. H., Rooney, A. P., Donnell, K., Trail, F., Gardiner, D. M., Manners, J. M., \& Kazan, K. (2013). Fusarium pathogenomics. Annual Review of Microbiology. 67: 399-416.

Maroyi, A. (2013). Traditional use of medicinal plants in south-central Zimbabwe: Review and perspectives. Journal of Ethnobiology and Ethnomedicine. 9 (31): 1-18.

Marufu, L. (2017). More companies fortify products. Chronicle, 12 July 2017. http://www.chronicle.co.zw/more-companies-fortify-products/.

Mathur, S. B., \& Olga, K. (2001). Common laboratory seed health testing methods of detecting fungi. Danish Government Institute of Seed Pathology for Developing Countries. Copenhagen: Kandrups Bogtrykkeri; Bassersdorf, Switzerland: International Seed Testing Association.

Maundu, P. M., Ngugi, G. W., \& Kabuye, C. H. S. (1999). Traditional food plants of Kenya. Kenya Resource Centre for Indigenous Knowledge, National Museums of Kenya.

Mnzava, N. A. (1997). Vegetable crop diversification and the place of traditional species in the tropics. In L. Guarino (Ed.), Traditional African Vegetables: Proceedings of the IPGRI International workshop on genetic resources of traditional vegetables in Africa: Conservation and use. International Plant Genetic Resources Institute.

Natarajan, B. (2002). Conservation of biological diversity and indigenous traditional knowledge as exemplified by the cases of Asia and Africa. African Journal of Indigenous Knowledge Systems. 1: 37-45.

Ngugi, I. K., Gitau, R., \& Nyoro, J. K. (2006). Access to high value markets by smallholder farmers of African Indigenous Vegetables in Kenya. Regoverning Markets Innovative Practice series. London: Sustainable Markets Group.

Ochieng, J., Afari-Sefa, V., Karanja, D. R., Srinivasulu, S., \& Samali Kessy, R. (2016). Promoting consumption of traditional African vegetables and its effect on food and nutrition security in Tanzania. Invited paper presented at the 5th International Conference of 
the African Association of Agricultural Economists, Addis Ababa, Ethiopia, 23-26 September.

Odhav, B., Beekrum, S., Akula, U., \& Baijnath, H. (2007). Preliminary assessment of nutritional value of traditional leafy vegetables in KwaZulu-Natal, South Africa. Journal of Food Composition and Analysis. 20: 430-435.

Ojiewo, C., Tenkouano, A., Oluoch, M., \& Yang, R. (2010). The role of AVRDC - The World Vegetable Centre in vegetable value chains. African Journal of Horticultural Science. 3: $1-23$.

Pasquini, M. W., Weinberger, K., Assogba-Komlan, F., Kouame, C., Akplogan, F., Djidji, H., Achigan-Dako, E. G., \& Young, E. M. (2014). Characterising urban and peri-urban production systems for African indigenous vegetables in four cities in Benin and Cote d'Ivoire. Acta Horticulturae. 1021: 137-151.

Ruel, M. T., Minot, N., \& Smith, L. (2005). Patterns and determinants of fruit and vegetable consumption in Sub-Saharan African: A multicountry comparison. Washington, DC: World Health Organization International Food Policy Research Institute.

Schaad, N. W., Jones, J. B., \& Chun, W. (Eds.) (2001). Laboratory guide for identification of plant pathogenic bacteria. $3^{\text {rd }}$ ed. St. Paul, MN: American Phytopathological Society.

Schippers, R. R. (1997). Domestication of indigenous vegetables for Sub-Saharan Africa: A strategy paper. In R. R. Schippers \& L. Budd (Eds.), African Indigenous Vegetables. Workshop proceedings, Limbe, Cameroon, 13-18 January (pp. 125-135). London: ODA.

Schippers, R. R. (2000). African indigenous vegetables: An overview of the cultivated species. Natural Resources Institute/ACP-EU Technical Centre for Agricultural and Rural Cooperation. Chatham: Natural Resources Institute, University of Greenwich.

Smith, I. F., \& Eyzaguirre, P. (2007). African leafy vegetables their roles in the World Health Organization's global fruit and vegetables initiative. African Journal of Food Agriculture Nutrition Development. 7 (3): 1-17.

Sogbohossou, E. O. D., Achigan-Dako, E. G., Maundu, P., Solberg, S., Deguenon, E. M. S., Mumm, R. H., Hale, I., Van Deynze, A., \& Schranz, M. E. (2018). A roadmap for breeding orphan leafy vegetable species: A case study of Gynandropsis gynandra (Cleomaceae). Horticulture Research. 5 (2): 1-15.

Tadele, Z. (2009). Role of orphan crops in enhancing and diversifying food production in Africa. African Technology Development Forum Journal. 6 (3/4): 9-16. 
Tata, P. I., Afari-Sefa, V., Ntsomboh-Ntsefong, G., Ngome, A. F., Okolle, N. J., \& Billa, S. F. (2016). Policy and institutional frameworks impacting on vegetable seed production and distribution systems in Cameroon. Journal of Crop Improvement. 30 (2): 196-216. Weinberger, K., \& Msuya, J. (2004). Indigenous vegetables in Tanzania-Significance and prospects. AVRDC Publication 04-600. Technical Bulletin No. 31. Shanhua: World Vegetable Center.

Weinberger, K., \& Pichop, G. N. (2009). Marketing of African indigenous vegetables along urban and peri-urban supply chains in Sub-Saharan Africa. In C. M. Shackleton, M. W. Pasquini, \& A. W. Drescher (Eds.), African indigenous vegetables in urban agriculture (pp. 225-244). London: Earthscan.

World Health Organization (WHO) (2012). Obesity and overweight. WHO Fact Sheet number 311. Geneva: World Health Organization.

Yang, R., \& Keding, G. B. (2009). Nutritional contributions of important African indigenous vegetables. In C. M. Shackleton, M. W. Pasquini, \& A. W. Drescher (Eds.), African indigenous vegetables in urban agriculture (pp. 105-143). London: Earthscan.

Zimbabwe Agenda for Sustainable Socio-Economic Transformation (ZimAsset), pdf. (2013). Government of Zimbabwe.

Zimbabwe National Statistics Agency and ICF International. (2016). Zimbabwe demographic and health survey 2015: Key indicators. Rockville, MD: Zimbabwe National Statistics Agency (ZIMSTAT); ICF International 
2 Seed health tests of traditional leafy vegetables and pathogenicity in plants ${ }^{1}$

1 This chapter is based on the article: Praxedis Dube, Paul C. Struik and Elizabeth Ngadze (2018). Seed health tests of traditional leafy vegetables and pathogenicity in plants. Published in African Journal of Agricultural Research DOI: 10.5897/AJAR2018.13001. 


\section{Abstract}

Traditional leafy vegetables play a significant role in the daily diets of many people in Zimbabwe. They are produced by smallholder farmers with limited knowledge on the agronomic value of high-quality seed, on seed storage, and on plant- and seed-borne pathogens. The quality of the seed is rarely tested for seed-borne pathogens posing risks of pathogen build-up. This study was conducted in order to determine the seed-borne pathogens associated with traditional leafy vegetable seeds. Seeds were collected from five different farmers in three provinces of Zimbabwe and tested for the presence of pathogens in the plant pathology laboratory of the University of Zimbabwe. A total of 154 fungal and 233 bacterial infections were recorded on 450 seeds for each type of pathogen. Significantly high percentages of the isolates (Xanthomonas campestris, Curvularia spp., Alternaria alternata, Fusarium oxysporum and Pseudomonas syringae) were obtained from the three different species of traditional leafy vegetables (Amaranthus hybridus, Bidens pilosa and Cleome gynandra). Fusarium oxysporum, Alternaria alternata, Xanthomonas campestris and Bacillus spp. isolates were pathogenic to traditional leafy vegetable plant species as confirmed by pathogenicity tests, hence may have significant effects on traditional leafy vegetables production. The results indicate that seed sourced from Farmers 1, 2, 3 and 4 resulted in seedborne pathogens on both the seed and plants, clearly showing that seed quality was poor. Although most pathogens observed have a broad host spectrum, pathogenicity and virulence tests confirmed that some pathogens were likely to be closely associated with these traditional leafy vegetable seed species. 


\subsection{Introduction}

Traditional leafy vegetables (TLVs) of Africa refer to plant species, which originated on the continent and have a long history of cultivation, domestication and use in African conditions (Ambrose-Oji, 2009). Amaranthus spp. combine both social and socio-economic importance in Kenya and Tanzania as a multi-purpose vegetable with environmental adaptability, nutritional, medicinal and income generation properties (Weinberger \& Msuya, 2004; Abukutsa-Onyango, 2007) and have gained popularity also in Zimbabwe. Mukwereza (2002) reported spider flower as the most preferred TLV in Zimbabwe. Together with Bidens pilosa and Cleome gynandra (spider flower) Amaranthus spp. also combine resilience to climate change, nutritional, medicinal and income generation properties.

Across Africa TLVs are produced and marketed by smallholder farmers (Ngugi et al., 2007) with limited access to advanced agro-technology including limited knowledge of the agronomic value of high-quality seed (Biemond et al., 2012), lack of information on seed storage (Cernansky, 2015), on appropriate use of chemicals like pesticides and fertilizers (Schippers, 2000), plants and seed-borne pathogens. These smallholder farmers obtain most of their seeds from their neighbours or informal seed markets (Ngwerume \& Mvere, 2003; Adebooye et al., 2005; Guei et al., 2011). This seed is most likely to be of poor quality. Seed is rarely tested for germination and seed-borne pathogens, increasing risks of seed degeneration across generations. Seed-borne pathogens can prevent germination and cause serious threats to seedling establishment (Valkonen \& Koponen, 1990; Walcott, 2003) and plant vigour (Anjorin \& Mohammed, 2014). Farmers using poor-quality seed risk poor germination and the ultimate crop stand in their fields will be poor. This may also affect crop yields and the quality of their vegetables on the market (Biemond et al., 2012; Mancini \& Romanazzi, 2013).

Most TLVs are propagated by seed (Grubben \& Denton, 2004). However, seed is the most important vehicle for transmitting plant pathogens globally (Kuan, 1988). Plant pathogens are reported to have close associations with seed, as they can survive in, on or with seed, hence can be preserved in seed lots (Copeland \& McDonald, 2001), at times in latent stage, are temperature dependent (Ngadze \& Icishahayo, 2014), and manifest themselves and become 
problematic only when environmental conditions become conducive for their growth and reproduction (Copeland \& McDonald, 2001). Seed has the potential to carry a wide range of pathogens including fungi, bacteria, viruses, many of which cause diseases in various plant developmental stages (from seedling to the product on the markets) (Copeland \& McDonald, 2001).

A seed health test is an important component for determining the quality of seed worldwide, a prerequisite to minimise losses by assessing the quality of seed before it is sown (Copeland \& McDonald, 2001). Seed health tests have been done on various seeds including tree seed, vegetable seed, and cereal seed (International Seed Testing Association - Seed Health (ISTA), 2015). Relatively very limited research on TLV seed has been reported (Sharma et al., 1980) including research on seed health tests, posing risks with seed germination (Cernansky, 2015). Plants are frequently left in the field for too long, risking seed-borne pathogens accumulation on seed (Adebooye et al., 2005).

TLV seed is affected by several seed-borne pathogens such as Rhizoctonia spp., Sclerotinia spp., Pythium spp., Choanephora cucurbitarum, Alternaria spp., Mycoplasma, Cercospora spp., Sphaerotheca fuliginea, and Oidiopsis taurica (Sharma et al., 1980; Grubben \& Denton, 2004) and some TLVs are important hosts of Xanthomonas campestris pv. campestris (Bradbury, 1986), which is also an important seed-borne pathogen of many plant species. Economically important examples of these seed-borne pathogens include Pythium spp., Pythium aphanidermatum and Rhizoctonia spp. causing a serious damping off problem in the Amaranthus hybridus seedbed, Cercospora bidentis and Cercospora uramensis affecting Bidens pilosa and Cleome gynandra, respectively (Grubben \& Denton, 2004). Cleome gynandra seed is also affected by a range of seed-borne fungi including Sphaerotheca fuliginea and Oidiopsis taurica. These pathogens were reported to cause powdery mildew on Cleome gynandra plants (Grubben \& Denton, 2004).

In Zimbabwe over 75\% growers of TLVs collect and retain seeds for sowing in the next season (Schippers, 2000) and the quality of the seed is rarely known. Moreover, farmers rely on traditional knowledge that is passed on through generations and often use traditional cropping technologies (Schippers, 2000). Considering that most of the seed-borne pathogens 
affecting TLV seed including Xanthomonas campestris, Cercospora spp, Stemphylium solani and Pythium spp. cause serious problems in vegetable production in Zimbabwe (Handiseni et al., 2008), there is, therefore, a high risk of seed-borne infections. Moreover, Rothwell (1982) listed Entyloma bidentis, Sclerotium rolfsii, Sphaceloma spp., Uromyce bidenticola, Verticillium dahliae, Pseudomonas solanacearum and Oidium spp. as pathogens that affect Bidens pilosa, Amaranthus spp. and Cleome spp. in Zimbabwe. Primary inoculum can also arise from other sources like contaminated tools and equipment (Jones et al., 1986), some TLVs are important host of Xanthomonas campestris pv. campestris (Bradbury, 1986), plant debris (Barak \& Liang, 2008). Nevertheless, seed-borne inoculum remains of paramount importance for most pathogen infections (Naseri \& Mousavi, 2015).

Generally, seed pathogens transferred to plants during growth cause considerable loss of crop yields globally (Copeland \& McDonald, 2001), but limited literature on the economic losses caused by these pathogens on TLVs have been reported. For example, Ngadze (2014) reported that some plant diseases may affect the plants during the growing phase and later stages in storage as well. The pathogens which are seed-borne affect the whole TLV food chain. Initially starting with pathogen infected seed, the pathogens may result in poor crop stand due to poor germination or poor-quality seedlings which cannot withstand relative environmental changes- dying away of plants. In storage and on the markets, the quality of the product is also affected by the pathogens including those passed through the use of infected seed-borne. This may result in food and nutrition insecurity and loss of incomes to the farmers, if little attention is paid to controlling seed-borne pathogens.

The research aims at detection and identification of seed-borne pathogens on TLV seed and establishes the relationship between seed infection and pathogen which develop on the plants in the field. In addition, the study seeks to ignite researches on the diseases and their economic impact on TLV seed. 
The specific objectives are:

- to identify seed-borne pathogens in TLV seed from different sources;

- to investigate whether pathogens found on seed are pathogenic to the plants.

Therefore the hypotheses tested include:

- Seed-borne pathogens' presence varies among seed sources;

- Seed-borne pathogens cause the diseases observed on the leaves of infected plants. 


\subsection{Methodology and Data Analysis}

The study uses three traditional leafy vegetable seed namely Cleome gynandra, Bidens pilosa and Amaranthus hybridus as examples. Cleome gynandra and Amaranthus hybridus are $\mathrm{C}_{4}$ plants (Imbamba et al. 1977; Schippers, 2000), while Bidens pilosa is a C 3 plant. Traditional leafy vegetables have a significant role in the traditional diets of many people in Zimbabwe.

\section{Origin of the seed sources}

Seed of the three species was sourced from five different farmers. These farmers were chosen using a snow ball sampling technique, with the entry point as the traditional leafy vegetable markets. Only farmers who had seed stocks from the previous season showing that they had been producing TLV seed were chosen. About 500 grams of seed for each species was sourced from the farmers and used for seed health tests. One farmer was a government research institute, i.e. Horticultural Research Centre (HRC). Lot sampling was done for seed sourced from HRC because they had bigger quantities in their stock than required and hand sampling was used to come up with approximately 500 grams of seed per each species. For the remaining other seed sources, all the seed available was sourced and most of the seed lots weighed less than $1 \mathrm{~kg}$ per species per source. The seed collection constituted of 15 samples (three samples of three species per seed source). Subsampling to approximately 400 seeds per sample was done using the hand halving method (International Seed Testing Association [ISTA], 2016). The seed was sourced from three different agro ecological regions of Zimbabwe: Mashonaland Central, Mashonaland West and Mashonaland East. These provinces (agro ecological regions) are some of the major vegetable growing ones of Zimbabwe, because of their proximity to major cities and because they are located in areas of high agricultural potential. Our research used only five seed sources and intended to ignite more research on these vegetables across Zimbabwe. The climatic conditions during crop growth and geographical coordinates of the seed origin sites are presented in Table 2. 
Table 2 Geographical coordinates and climatic data during crop growth of the origin sites of the seed

\begin{tabular}{|c|c|c|c|c|c|c|c|c|}
\hline Farmer & Origin & $\begin{array}{l}\text { Agro } \\
\text { ecological } \\
\text { region }^{\text {a }}\end{array}$ & $\begin{array}{l}\text { Mean annual } \\
\text { temperature }\end{array}$ & $\begin{array}{l}\text { Temperature } \\
\text { range during } \\
\text { crop growth }\end{array}$ & $\begin{array}{l}\text { Geographical } \\
\text { coordinates }\end{array}$ & $\begin{array}{l}\text { Altitude } \\
\text { (m) }\end{array}$ & $\begin{array}{l}\text { Annual } \\
\text { rainfall } \\
(\mathrm{mm})\end{array}$ & $\begin{array}{l}\text { Amount of } \\
\text { rainfall } \\
\text { during crop } \\
\text { growth } \\
(\mathrm{mm})\end{array}$ \\
\hline 1 & $\begin{array}{l}\text { Mashonaland } \\
\text { West }\end{array}$ & $2 B$ & $22-29^{\circ} \mathrm{C}$ & $31-22^{\circ} \mathrm{C}$ & 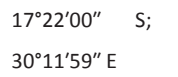 & 1153 & $700-800$ & $73-0.31$ \\
\hline 2 & $\begin{array}{l}\text { Mashonaland } \\
\text { East }\end{array}$ & 3 & $18-29^{\circ} \mathrm{C}$ & $31-21^{\circ} \mathrm{C}$ & $\begin{array}{l}17^{\circ} 38^{\prime} 35.59^{\prime \prime} \\
S ; 31^{\circ} 47^{\prime} 2.40^{\prime \prime} \\
\text { E }\end{array}$ & 1400 & $800-850$ & $100-0.8$ \\
\hline 3 & $\begin{array}{l}\text { Mashonaland } \\
\text { East }\end{array}$ & 3 & $18-29^{\circ} \mathrm{C}$ & $31-21^{\circ} \mathrm{C}$ & $\begin{array}{l}17^{\circ} 38^{\prime} 35.59^{\prime \prime} \\
\mathrm{S} ; 31^{\circ} 47^{\prime} 2.40^{\prime \prime} \\
\text { E }\end{array}$ & 1400 & $800-850$ & $100-0.8$ \\
\hline 4 & $\begin{array}{l}\text { Mashonaland } \\
\text { Central }\end{array}$ & 3 & $21-31^{\circ} \mathrm{C}$ & $31-21^{\circ} \mathrm{C}$ & $\begin{array}{l}17^{\circ} 18^{\prime} 56^{\prime \prime} \mathrm{S} ; \\
31^{\circ} 34^{\prime} 14^{\prime \prime} \mathrm{E}\end{array}$ & 953 & $800-850$ & $110-1.5$ \\
\hline 5 & $\begin{array}{l}\text { Mashonaland } \\
\text { East }\end{array}$ & $2 \mathrm{~B}$ & $18-29^{\circ} \mathrm{C}$ & $29-27^{\circ} \mathrm{C}$ & $\begin{array}{l}18^{\circ} 11^{\prime} 6.97^{\prime \prime} \mathrm{S} ; \\
\left.31^{\circ} 33^{\prime} 6.95^{\prime \prime} \mathrm{E}\right)\end{array}$ & 1688 & $850-900$ & $105.7-24.8$ \\
\hline
\end{tabular}

a. Note: Agro ecological classification also known as natural region (NR) classification of Zimbabwe, divided the country into five regions based on mean annual rainfall and was done in the 1960s (Vincent \& Thomas, 1960).

b. Source: authors, https://www.worldweatheronline.com Historical average weather 20/04/2017 (for full details see Annex 1 and 2 description)

\section{Detection of seed-borne pathogens}

Seeds of the three TLV types were randomly selected and 150 seeds of each type of pathogen were plated on potato dextrose agar (PDA) and nutrient agar (NA) for isolation of fungi and bacteria respectively. The seeds were surface sterilized in 1\% sodium hypochorite and rinsed three times with sterile distilled water. The sterile seeds were blotted with sterile filter paper (Whatman no.1) to remove excess water prior to plating. A total of 10 seeds were plated out on a $90 \mathrm{~mm}$ diameter petri dish. Seeds for fungal identification were plated on PDA while those for bacteria were placed on NA plates. Each Petri dish was replicated 6 times (3 plates bacteria and 3 for fungi). The Petri dishes with seeds on PDA were incubated at $25 \pm 2^{\circ} \mathrm{C}$ under $12 / 12$ alternating cycles of NUV and darkness in Plant Pathology incubation room at the University of Zimbabwe for seven days. The seeds on NA were placed in an incubator (Gallenkemp- Sussex, England) and maintained at $30 \pm 2^{\circ} \mathrm{C}$ for 4 days. After incubation the seeds were viewed under a stereo microscope using the $16 \times$ objective. 


\section{Identification of fungi}

After the incubation period the fungi, which grew on the seeds, were examined and identified under stereomicroscope (WILD M3B, Heerbrugg, Switzerland) based on the growth characters of the fungi. Slides were prepared using the associated fungi on seeds for identification under a compound microscope (JENA LABOVAL, Göttingen, Germany) for better identification following the keys described by Mathur and Olga (2001) and Commonwealth Mycological Institute descriptions.

\section{Identification of bacteria}

After the incubation period, further plating was done on the culture media, colonies of bacteria were purified on the solidified potato dextrose agar or nutrient agar, respectively, were then incubated at $25^{\circ} \mathrm{C}$ for 48 hours. The morphological identification of the spores for fungi and bacteria was performed according to Mathur \& Olga (2001) and Schaad et al. (2001).

Single colonies of each isolate were used for physiological and biochemical tests. The isolates were identified by standard bacterial methods based on Cother and Sivasithamparam (1983). The tests performed were Gram reaction, oxidase activity, glucose metabolism, pectate degradation in Sutton's medium, production of phosphatase, indole production from tryptophan, gelatine hydrolysis, production of reducing substances from sucrose, production of acid from $\alpha$-methylglucoside and trehalose, malonate utilisation, sensitivity to erythromycin $(15 \mu \mathrm{g})$, growth at $37^{\circ} \mathrm{C}$ which was determined after $24 \mathrm{~h}$ in nutrient broth (NB, Difco) and salt tolerance which was checked after $48 \mathrm{~h}$ growth in NB with $5 \mathrm{~g} \mathrm{l}^{-1} \mathrm{NaCl}$.

\section{Detection and identification of pathogens on infected plants}

The aim was to investigate whether seed-borne pathogens on seed corresponded to disease symptoms observed on plants. Plants were grown in the greenhouse at the University of Zimbabwe in February of 2017. A randomized complete block design with three blocks was used. The pots were labelled and randomly arranged and filled to approximately $6 \mathrm{~cm}$ under the rim. The soil was sterilised at $70^{\circ} \mathrm{C}$ for 24 hours and allowed to cool. A mixture of soil and pine bark at ratio 1:3 was done to reduce soil compaction and placed in pots. Proper levelling of the growing media was done before approximately 1000 seeds were evenly distributed per pot. The seeds were then covered with $1.5 \mathrm{~cm}$ thick layer of the growing media. Water as 
needed was added to the pots. The presence of the diseases on plants was also recorded in the greenhouse (e.g. damping off).

The infected plant parts showing typical symptoms of diseases were collected for further analysis. Plant pathogens were isolated from different parts of the crops that had advanced lesions and rots. The lesions and surrounding healthy tissues were cut into small pieces and washed in running tap water before sterilizing with $1 \%$ sodium hypochlorite for $2-3$ minutes, followed by rinsing three times in sterile distilled water, and drying on sterile blotter papers. Disinfected pieces were placed on solidified Nutrient Agar (NA) medium (Schaad et al. 2001). The Petri dishes were incubated at $28 \pm 2^{\circ} \mathrm{C}$ and observed for bacterial growth 4-6 days later. After the incubation period, the further plating was done on the culture media, colonies of fungi and bacteria were purified on the solidified potato dextrose agar or nutrient agar, respectively, and were then incubated at $25^{\circ} \mathrm{C}$ for 48 hours. Thereafter, the morphological identification of the spores for fungi and bacteria was performed according to Mathur \& Olga (2001) and Schaad et al. (2001).

\section{Hypersensitivity}

Isolated colonies of different bacteria were tested for ability to induce a hypersensitive response on foliage of the non-host plant tobacco (Nicotiana tabacum cv. Burley). Five isolates were selected for each of the three bacteria species isolated from seed and infected plants. Test isolates were freshly grown on NA at $30 \pm 2^{\circ} \mathrm{C}$ for $24 \mathrm{~h}$ and suspended in sterile water, maintain an inoculums concentration of $10^{8} \mathrm{cfu} / \mathrm{ml}$ (using Genie spectrophotometer at $550 \mathrm{~nm}$ ). Tobacco seedlings were inoculated with suspensions of the selected isolates at the 5-6 leaf stage. $1 \mathrm{ml}$ of bacterial suspension was injected using a disposable syringe into the lower surface of the tobacco leaves and the plants were sealed in plastic bags for $24 \mathrm{~h}$ to prevent desiccation. Plants were uncovered and kept in the laboratory at $28 \pm 2^{\circ} \mathrm{C}$ for $36 \mathrm{~h}$. Control plants were injected with sterile distilled water and kept under same conditions. 
Virulence test

For the assessment of pathogenicity of the different bacteria on TLV seedlings, inoculum suspensions were prepared in sterile water as previously described for the hypersensitive test. Young TLV seedlings were inoculated with $1 \mathrm{ml}$ of inoculum using a hypodermic syringe. Control leaves were inoculated with sterile distilled water.

Data Analysis

Data was analysed using GenStat $18^{\text {th }}$ edition. The data was transformed using LOG10 plus 1 and subjected to analysis of variance (ANOVA) and the means of the parameters were separated using the Duncan's multiple range least significant difference test $(P \leq 0.0)$.5The transformation was done to normalise the data sets because some data sets had many zeros. 


\subsection{Results}

A total of 154 fungal and 233 bacterial infections were recorded on 450 seeds (for each type of pathogen) selected from seeds of three types of TLV. The fungi were identified based on colony morphologies as Curvularia spp., Alternaria alternata, Fusarium oxysporum, Aspergillus spp., Phoma betae, Colletotrichum spp. and Rhizoctonia solani. Three bacteria species were isolated from the seed and they were identified as Xanthomonas campestris, Pseudomonas syringae and Bacillus spp. The seed-borne infections varied according to seed species and source. The virulence test conducted on young TLV seedlings varied by pathogen species and seed species.

\section{Hypersensitivity test}

Table 3 shows hypersensitivity responses of the different isolates tested on tobacco. Ten of the fifteen isolates tested on tobacco plants induced a hypersensitivity test within 24-48 hours after inoculation and based on these reactions they were categorized as strongly, moderately, or mildly virulent. All isolates were considered as pathogenic since they produced a reaction within 72 hours after inoculation. Xanthomonas campestris isolates 1, 3 and 5, Pseudomonas syringae isolates 3, 4 and 5 as well as Bacillus spp. isolates 3 and 4 were regarded as highly virulent. Two isolates Xanthomonas campestris 4 and Bacillus spp. 1 were moderately virulent while isolates Xanthomonas campestri 2, Pseudomonas syringae 1 and 2 as well as Bacillus spp. 1 and 5 were considered to be weakly virulent. 
Table 3 Hypersensitive responses of the different isolates tested on tobacco

\begin{tabular}{|c|c|c|c|c|}
\hline Isolate & Source & Hypersensitive response (+/-) & Duration (hrs) & Virulence \\
\hline Xanthomonas campestris 1 & seed & + & 24 & High \\
\hline Xanthomonas campestris 2 & plant & + & 36 & Weak \\
\hline Xanthomonas campestris 3 & seed & + & 36 & High \\
\hline Xanthomonas campestris 4 & seed & + & 24 & Moderate \\
\hline Xanthomonas campestris 5 & plant & + & 72 & High \\
\hline Pseudomonas syringae 1 & seed & + & 36 & Weak \\
\hline Pseudomonas syringae 2 & seed & + & 24 & Weak \\
\hline Pseudomonas syringae 3 & plant & + & 48 & High \\
\hline Pseudomonas syringae 4 & plant & + & 72 & High \\
\hline Pseudomonas syringae 5 & plant & + & 72 & High \\
\hline Bacillus spp. 1 & seed & + & 24 & Moderate \\
\hline Bacillus spp. 2 & plant & + & 72 & Weak \\
\hline Bacillus spp. 3 & seed & + & 72 & High \\
\hline Bacillus spp. 4 & seed & + & 24 & High \\
\hline Bacillus spp. 5 & plant & + & 36 & Weak \\
\hline Control & distilled water & - & $24-72$ & - \\
\hline
\end{tabular}

\section{Virulence tests}

All isolates gave a positive response on all three TLV plant species (Table 4). The isolates were ranked as highly, moderately and weakly virulent pathogens based on host responses. Among these highly virulent isolates, symptoms in plants inoculated with Xanthomonas campestris isolates 1, 3 and 4 were highly virulent on Bidens pilosa and Cleome gynandra and moderately virulent on Amaranthus hybridus and the symptoms developed earlier than with other isolates. Plants inoculated with these isolates showed disease symptoms within 6 days. Pseudomonas syringae isolates 3 and 4 were highly virulent on Bidens pilosa and Amaranthus hybridus. No symptoms developed in controls treated with sterile water. 
Table 4 Virulence of the different isolates on seedlings of traditional leafy vegetables

$\begin{array}{lccc}\text { Isolate } & \text { Amaranthus hybridus } & \text { Cleome gynandra } & \text { Bid } \\ \text { Xanthomonas campestris } 1 & ++ & ++ & ++ \\ \text { Xanthomonas campestris } 2 & ++ & + & + \\ \text { Xanthomonas campestris } 3 & ++ & + & ++ \\ \text { Xanthomonas campestris } 4 & ++ & ++ & ++ \\ \text { Xanthomonas campestris } 5 & ++ & ++ & ++ \\ \text { Pseudomonas syringae } 1 & ++ & ++ & + \\ \text { Pseudomonas syringae 2 } & ++ & + & ++ \\ \text { Pseudomonas syringae 3 } & +++ & ++ & ++ \\ \text { Pseudomonas syringae } 4 & ++ & ++ & ++ \\ \text { Pseudomonas syringae } 5 & ++ & ++ & ++ \\ \text { Bacillus spp. } 1 & ++ & ++ & ++ \\ \text { Bacillus spp. } 2 & + & ++ & ++ \\ \text { Bacillus spp. } 3 & + & ++ & + \\ \text { Bacillus spp. } 4 & ++ & + & ++ \\ \text { Bacillus spp. } 5 & ++ & ++ & ++ \\ \text { Control } & - & - & -\end{array}$

a. Note:,+++++ and + denote highly, moderately and weakly virulent pathogens, respectively

Isolation of pathogens from seed by plant species and source

The percentage occurrence of seed-borne pathogens for plant species and seed farmers were evaluated and are presented in Table 5 and Table 6, respectively. For both cases, the percentage occurrence refers to the frequency of any single isolate observed on each seed which is measured as the proportion of the seeds per species that are infected by seed-borne pathogens. The percentage occurrence was calculated by adjusting Jager et al. (2007) calculations as number of seeds having the pathogen during the analysis divided by the total number of seeds used per species and the ratio is multiplied by 100 (following formula used) and subsequently used to determine the differences by performing the analysis of variance and separation of means using Duncan's multiple range method in GenStat.

$$
\text { Percentage occurrence }=\frac{\text { No. of seeds affected by a pathogen }}{\text { Total no. of seeds }} \times 100
$$

Table 5 shows the percentage occurrence of seed-borne pathogens isolated from seeds of three species. In comparison among plant species, highest percentage occurrence of seedborne pathogens was observed on Cleome gynandra, followed by Bidens pilosa and least on Amaranthus hybridus seed. Only Xanthomonas campestris, Pseudomonas syringae and Alternaria alternata isolates were significantly different for the percentage occurrence of 
seed-borne pathogens on seed among the three plant species. For Xanthomonas campestris the percentage occurrence of seed-borne pathogens on seed for Cleome gynandra and Bidens pilosa did not differ from each other, which was significantly higher than for Amaranthus hybridus. On the other hand, Cleome gynandra showed the lowest percentage occurrence on Pseudomonas syringae. For both plant species, the percentage occurrence of Alternaria alternata on seed was highest on Cleome gynandra, intermediate on Bidens pilosa and lowest on Amaranthus hybridus.

Table 5 The percentage occurrence of seed-borne pathogens on seed for three plant species (between brackets the transformed values and the mean separation)

\begin{tabular}{|c|c|c|c|c|}
\hline \multirow[b]{2}{*}{ Name of pathogen } & \multicolumn{3}{|c|}{ Plant species } & \multirow[t]{2}{*}{$L S D_{5 \%}$} \\
\hline & Amaranthus hybridus & Cleome gynandra & Bidens pilosa & \\
\hline \multicolumn{5}{|l|}{ Bacteria } \\
\hline Xanthomonas campestris & $1.3(0.09 a)$ & $79.3(1.60 b)$ & $58.7(1.20 b)$ & 0.57 \\
\hline Pseudomonas syringae & $6.7(0.36 a b)$ & $0.0(0.00 \mathrm{a})$ & $8.7(0.53 b)$ & 0.40 \\
\hline Bacillus spp. & 0.7 (0.07a) & $0.0(0.00 \mathrm{a})$ & $0.0(0.00 a)$ & 0.11 \\
\hline \multicolumn{5}{|l|}{ Fungi } \\
\hline Alternaria alternata & $1.3(0.14 a)$ & $18.7(1.12 \mathrm{c})$ & $10.7(0.74 b)$ & 0.38 \\
\hline Aspergillus spp. & $0.0(0.00 \mathrm{a})$ & $0.0(0.00 \mathrm{a})$ & 4.7 (0.19a) & 0.22 \\
\hline Fusarium oxysporum & $12.0(0.57 a)$ & $3.3(0.18 a)$ & $1.3(0.14 a)$ & 0.41 \\
\hline Curvularia spp. & $2.7(0.23 a)$ & $25.3(0.78 a)$ & $18.7(0.69 a)$ & 0.55 \\
\hline Phoma betae & $0.0(0.00 \mathrm{a})$ & $0.0(0.00 \mathrm{a})$ & $1.3(0.09 a)$ & 0.15 \\
\hline Colletotrichum spp. & $0.0(0.00 \mathrm{a})$ & $1.3(0.14 a)$ & $0.0(0.00 \mathrm{a})$ & 0.16 \\
\hline Rhizoctonia solani & $1.3(0.09 a)$ & $0.0(0.00 \mathrm{a})$ & $0.0(0.00 a)$ & 0.15 \\
\hline
\end{tabular}

The mean separation indicates per pathogen the differences among the three species $(P \leq 0.0) .5$

Table 6 shows the percentage occurrence of seed-borne pathogens per farmer. The percentage occurrence of a single bacterial or fungal isolate differed among the farmers but only significantly for Curvularia spp. and Colletotrichum spp. The lowest percentage occurrence of Curvularia spp. was detected for Farmer 5, followed in order by Farmers 3, 2, and 4 and highest for Farmer 1. For Colletotrichum spp. the highest percentage occurrence of a single isolate was observed for Farmer 2, and the remaining farmers were not significantly different from each other. 
Table 6 The percentages occurrence of seed-borne pathogens per seed farmer (between brackets the transformed values and the mean separation)

\begin{tabular}{|c|c|c|c|c|c|c|}
\hline \multirow[t]{2}{*}{ Name of pathogen } & \multicolumn{5}{|c|}{ Seed source } & \multirow[t]{2}{*}{$L S D$} \\
\hline & Farmer 1 & Farmer 2 & Farmer 3 & Farmer 4 & Farmer 5 & \\
\hline \multicolumn{7}{|l|}{ Bacteria } \\
\hline Xanthomonas campestris & $35.7(0.82 a)$ & $64.4(1.33 a)$ & $66.7(1.34 a)$ & $33.3(0.67 a)$ & $32.2(0.66 a)$ & 0.95 \\
\hline Pseudomonas syringae & 3.3 (0.17a) & $2.2(0.15 a)$ & $7.8(0.44 a)$ & $6.7(0.33 a)$ & $5.6(0.41 a)$ & 0.56 \\
\hline Bacillus spp. & $0.0(0.00 a)$ & $0.0(0.00 a)$ & $0.0(0.00 a)$ & $0.0(0.00 a)$ & $1.11(0.12 a)$ & 0.15 \\
\hline \multicolumn{7}{|l|}{ Fungi } \\
\hline Alternaria alternata & $7.8(0.64 a)$ & 16.7 (0.93a) & $10.0(0.67 a)$ & $10.0(0.58 a)$ & $6.7(0.53 a)$ & 0.64 \\
\hline Aspergillus spp. & $0.0(0.00 a)$ & $0.0(0.00 a)$ & $0.0(0.00 a)$ & $6.7(0.20 a)$ & $1.1(0.12 a)$ & 0.29 \\
\hline \multirow[t]{2}{*}{ Fusarium oxysporum } & $11.1(0.38 a)$ & $2.2(0.23 a)$ & $0.0(0.00 a)$ & $6.7(0.41 a)$ & $7.8(0.45 a)$ & 0.55 \\
\hline & & & 30.0 & & & \\
\hline Curvularia spp. & $41.1(1.32 c)$ & $3.3(0.35 \mathrm{ab})$ & $(0.81 \mathrm{bc})$ & $3.3(0.35 a b)$ & $0.0(0.00 a)$ & 0.61 \\
\hline Phoma betae & 0.0 (0.00a) & $0.0(0.00 a)$ & $0.0(0.00 a)$ & $2.2(0.15 a)$ & $0.0(0.00 a)$ & 0.19 \\
\hline Colletotrichum spp. & $0.0(0.00 a)$ & $2.2(0.23 b)$ & $0.0(0.00 \mathrm{a})$ & $0.0(0.00 \mathrm{a})$ & $0.0(0.00 \mathrm{a})$ & 0.20 \\
\hline Rhizoctonia solani & $2.2(0.15 a)$ & $0.0(0.00 \mathrm{a})$ & $0.0(0.00 \mathrm{a})$ & $0.0(0.00 \mathrm{a})$ & $0.0(0.00 \mathrm{a})$ & 0.33 \\
\hline
\end{tabular}

The mean separation indicates per pathogen the differences among the five farmers $(P \leq 0.0) .5$

The two-way interactions between farmer and TLV species on percentage occurrence of seedborne pathogens (for full results see Annex 3 to Annex 12)

The difference between TLV species in the percentage occurrence of the Xanthomonas campestris isolate (Annex 3 ) depends on the farmer, with Farmer 1 showing occurrences very different from the other farmers. Similarly, a two-way ANOVA carried out on the Pseudomonas syringae isolate (Annex 4) showed that the difference in occurrence between TLV species depended on the farmer, with Amaranthus hybridus and Bidens pilosa showing much more variation among farmers than Cleome gynandra. For the Bacillus spp. isolate (Annex 5), the results showed that the ranking of the species for each farmer was only different for Farmer 5. The results of a two-way ANOVA on the Alternaria alternata isolate (Annex 6) depicted that the order of occurrence of the disease among the TLV species strongly depended on species and varied considerably. Similarly, for the Aspergillus spp. isolate (Annex 7) the results showed that the difference between farmers depended on TLV species with Farmer 4 showing the largest differences. For the two-way ANOVA carried out for the Fusarium oxysporum isolate (Annex 8), the findings showed that the differences among, but also the ranking of the TLV species were different for each farmer. The results for the Curvularia spp. isolate (Annex 9) also showed that both the differences among and the ranking of the TLV species differed among farmers. For the Phoma betae isolate (Annex 10), the 
ranking of the TLV species was consistent among the farmers, but the extent of the differences among the species differed among farmers. A two-way ANOVA was also carried out to assess the interaction between farmer and TLV species on the Colletotrichum spp. isolate (Annex 11), demonstrating that the variation among TLV species was larger for Farmer 2 than for the other farmers. For the Rhizoctonia solani isolate (Annex 12), Farmer 1 showed the largest difference among TLV species, especially because Amaranthus hybridus had a high value.

\section{Pathogenicity tests}

Table 7 depicts the occurrence of seed- and soil-borne pathogens on plants. The occurrence here refers to the presence of seed-borne pathogens on plant parts (showing typical symptoms of diseases) and were not scored or scaled. For pathogenicity tests of seed-borne diseases on plants, two bacterial and six fungal species were identified. The two bacterial species included Xanthomonas campestris and Bacillus spp. while the fungal species were Alternaria alternata, Fusarium oxysporum, Trichoderma spp., Penicillium spp., Erysiphe spp. and Pythium spp. Several soil pathogens including Bacillus spp., Trichoderma spp. and Penicillium spp. were also isolated from the plant parts. The farmers with an asterisk indicate that the observed pathogen were also present on seed.

Table 7 The occurrence of seed and soil-borne pathogens on plants

\begin{tabular}{|c|c|c|c|}
\hline \multirow[b]{2}{*}{ Name of pathogen } & \multicolumn{3}{|c|}{ Plant species } \\
\hline & Amaranthus hybridus & Cleome gynandra & Bidens pilosa \\
\hline \multicolumn{4}{|l|}{ Bacteria } \\
\hline Xanthomonas campestris & Absent & Present (Farmer 2)* & Absent \\
\hline Bacillus spp. & Absent & Present (Farmer 4) & Present (Farmer 2) \\
\hline \multicolumn{4}{|l|}{ Fungi } \\
\hline Alternaria alternata & Present (Farmer 1$)^{*}$ & Absent & Present (Farmer 3)* \\
\hline Fusarium oxysporum & Present (Farmer 1$)^{*}$ & Present (Farmer 4)* & Present (Farmer 5) \\
\hline Penicillium spp. & Present (Farmer 1) & Present (Farmer 2) & Present (Farmer 2) \\
\hline Trichoderma spp. & Absent & Present (Farmer 4) & Absent \\
\hline Erysiphe spp. & Absent & Absent & Present (Farmer 3) \\
\hline Pythium spp. & Present (Farmer 4) & Absent & Absent \\
\hline
\end{tabular}
a. The words in parentheses represent the farmers
b. * observed pathogens were also present on seed 
Comparison of seed-borne pathogens on seed corresponds to pathogenicity on plants The pathogenicity tests on the plants indicated that Fusarium oxysporum and Penicillium spp. had a wide range of infections as they were observed on all the three species, followed by Alternaria alternata and Bacillus spp. as these pathogens were present on two species while Xanthomonas campestris, Trichoderma spp., Erysiphe spp. and Pythium spp. had the least pathogenicity range, present only on one species. Occurrence of Xanthomonas campestris, Alternaria alternata and Fusarium oxysporum on both seed and plants was observed. Occurrence of seed-borne pathogens on both the seed and plants was observed for Farmers 1, 2, 3 and 4 . 


\subsection{Discussion}

Seed heath testing is important for four reasons: reduce seed-borne inoculum incidences and give an economic value to the seed lot, its requirements to satisfy the seed movement, elucidate seedling evaluation and causes of poor germination and give recommendation and perform seed lot treatment in order to eradicate seed-borne pathogens and reduce the risk of disease transmission (ISTA, 2015).

This study aims at identifying seed-borne pathogens in TLV seed from different farmers. This is primarily useful since the literature on seed-borne pathogens for TLV seed is scanty and farmers normally rely on literature for other crops like exotic cabbages which are relatively different in terms of production requirements or they rely on traditional production knowledge that is passed on through generations (Schippers, 2000). Unlike cabbage seed from relatively improved hybrid varieties, TLV seed predominantly is from traditional unimproved varieties. Therefore, in Zimbabwe, their production requirements are totally different from cabbage and TLV farmers should not rely on information on cabbage. Furthermore, this study investigated whether pathogens found on seed showed pathogenicity to the plants. There is limited literature on pathogenicity tests for TLVs, hence this study aims at filling that gap.

Seed samples of three TLV plant species from five different farmers were compared for the percentage occurrence of seed-borne pathogen isolate on each seed. Furthermore, the study investigated whether pathogens found on seed show pathogenicity to the plants. Pathogenicity tests were conducted and showed pathogenicity effects on seeds and plants. Major pathogens included Xanthomonas campestris, Alternaria alternata and Fusarium oxysporum. The seed-borne pathogens Xanthomonas campestris and Alternaria spp. were also reported to be pathogenic to traditional leaf vegetables in several other studies (Sharma et al., 1980; Bradbury, 1986; Grubben \& Denton, 2004), but no such reports were made on Fusarium oxysporum. The results also indicated that seed sourced from Farmers 1, 2, 3 and 4 had occurrence of seed-borne pathogens on both the seed and plants (Table 7). These results clearly show that farmers use bad seed. Further, comparison on accumulative percentage occurrence for the 10 seed-borne pathogens observed (Table 6) showed wide ranges: Farmer 3 (114.4), followed by Farmer 1 (101.2), then, third, Farmer 2 (91.0), the fourth, Farmer 4 
(68.9) and least, Farmer 5 (54.51). The superiority of Farmer 5 over other farmers was expected, because it is a research organization, which has much better knowledge on seed production compared to its counterparts. On the other hand, differences in the percentage occurrence of seed-borne pathogens among the sources may partially be explained by different crop management practices during seed development as previously reported by Bradbury (1986) and Naseri \& Emami (2013). Bradbury (1986) reported that some TLVs are important hosts of Xanthomonas campestris pv. campestris, and, therefore, likely sources of inoculum if diseased plants are left to grow and subsequently seed is harvested.

Farmers need seed of good quality to start with, for them to be able to satisfy the market requirements. The quality of the seed is comprised of many different attributes that add to the performance of the seed. Being free from seed-borne diseases, is figured out the most important one (Dube \& Mujaju, 2013). Observations indicating that higher levels for percentage occurrence of seed-borne pathogen isolate on each seed by farmer (statistically significant at $5 \%$ ) is also confirmed by the pathogenicity tests. Although, there are not yet established the quality standards of these plant species in literature, farmers require clean seed, that is free from seed-borne diseases for them to increase their yields and get better incomes from their crops. Many seed-borne pathogens become active when seeds are sown, which may result in seed decay or pre- or post- emergence damping off (Agarwal \& Sinclair,

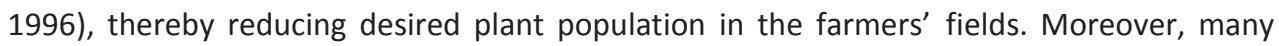
seed-borne pathogens, particularly, fungi induce qualitative changes in the physio-chemical properties of seeds such as protein content (Agarwal \& Sinclair, 1996) that are fundamental in the germination process. For instance, Mathur \& Sehgal (1964) reported that a collective of fungal pathogens contaminants, e.g. Fusarium spp., reduced seed vigour and inhibited seed germination. Notably, some isolates of Bacillus spp. decreased seed germination or seedling growth (Broadbent et al., 1977).

The observation of the pathogens on both seed and plants and moderate to high virulence on tested plant species may explain their strong association with seed and ability to give rise to progressive disease development in the field. Such developments influence the ultimate crop yield and reduce the commercial value of the crop as well (Naseri \& Mousavi, 2015). This may, therefore, give a positive indication of possible need of control measures. For example, in a 
study carried by Rude et al. (1999), infection of seed by Alternaria spp. greatly reduced seed germination of turnip. Although in this study we did not test the germination of seed, literature regarding these TLV seed has reported difficulties with seed germination (Cernansky, 2015) which cannot be overlooked the incidences of seed-borne as an important factor reducing the seed germination.

Virulence is the degree of pathogenicity exhibited by most of the pathogens and it is a measure that effectively differentiates pathogenic and non-pathogenic strains (Russell \& Herward, 2005). All bacteria isolates gave a positive response on all three TLV plant species (Table 4) and with varied magnitudes, ranking as highly, moderately and weakly virulent pathogens based on host responses. Interestingly, among the tested plant species for virulence, none were found to possess a high level of resistance to the present bacteria pathogens observed in the study. Differential virulence on these TLV plant species are further confirmed by pathogenicity test. These results suggest, although the observed pathogens have wide host range, these pathogens were sufficient to cause host-generated infections and if ignored may cause serious problems in TLV seed and vegetables production.

Erysiphe spp. were observed on Bidens pilosa during the late stage of growth, i.e. seed development and seed maturation. Erysiphe cruciferarum in wild radish has been shown to affect the crop late and is destructive at the seed development stage (Djébali et al., 2009). Schippers (2000); Grubben \& Denton (2004) also confirmed the presence of Alternaria spp. in East Africa causing damping off. Our results showed that the bacterium Xanthomonas campestris, although present in high quantities on seed of all three plant species, exhibited pathogenicity on Cleome gynandra only and did not show up on Amaranthus hybridus and Bidens pilosa plants. Xanthomonas campestris has already been reported as a serious pathogen, causing economic problems in vegetables production in Zimbabwe (Handiseni et al., 2008). Considering its close association with Cleome gynandra seed, it poses a serious threat in Cleome gynandra production as well. On the same note, Trichoderma spp. was isolated from one sample of Cleome gynandra and it was detected in all the replicates. The plants had leaf spot. Trichoderma spp. can behave as an opportunistic pathogen. Harman et al. (2004) confirmed that particular strains of Trichoderma spp. in rare cases, are pathogenic to plants and have been reported to cause diseases of the crops such as apples, maize and 
alfalfa. Some Trichoderma spp. strains also grow on leaf surfaces. That probably we detected Trichoderma spp. in one of our samples, may also pose a serious threat in Cleome gynandra production. Also, Bacillus spp. was isolated on all three plant species, Amaranthus hybridus seed and Cleome gynandra and Bidens pilosa. Some Bacillus spp. isolates can be beneficial, whereas others can be detrimental in crop production. Broadbent et al. (1977) reported that some Bacillus spp. increased plant growth by producing gibberellin or auxin or solubilizing phosphate, while others reduced germination. 


\subsection{Conclusions and Recommendations}

Although most of the pathogens observed in our results have a broad host spectrum, pathogenicity and virulence tests substantiate that some pathogens were more likely to be closely associated with these traditional leafy vegetable seed species. All bacteria isolates gave a positive response on all three TLV plant species and with varied magnitudes, ranking as highly, moderately and weakly virulent pathogens based on host responses. However, none of the plant species were found to possess a high level of resistance to the present bacteria pathogens observed in the study. Furthermore, Xanthomonas campestris, Fusarium oxysporum, Alternaria alternata and Bacillus spp. isolates were pathogenic to Amaranthus hybridus, Cleome gynandra and Bidens pilosa plants as confirmed by pathogenicity tests. The occurrence of seed-borne pathogens on both the seed and plants for Farmers 1, 2, 3 and 4 clearly showed bad seed.

One further important area for future research is virulence testing for fungal pathogens using more samples collected from various places around Zimbabwe. Such tests will lead to better understanding of pathogens affecting TLV seed and production, and to more knowledge on certain pathogens prevalent in certain areas. There is limited literature reported on the economic losses caused by these pathogens on TLVs.

\section{Acknowledgements}

This research was possible through the support of the Dutch Government through the Netherlands Fellowship Programme. Thank you to University of Zimbabwe Technicians, Mrs Matikiti, Sanyamuwera Martin and Magadhi Denver, you made my work more enjoyable. 


\section{References}

Abukutsa-Onyango, M. O. (2007). Seed production and support systems for African Leafy Vegetables in three communities in Western Kenya. African Journal of Food Agriculture Nutrition and Development. 7 (3): 1-16.

Agarwal, V. K. \& Sinclair, J. B. (1996). Principles of Seed Pathology. Second Edition. CRC Press, Lewis Publisher, Boca Raton, Florida. 1-515.

Adebooye, O. C., Ajayi, S. A., Baidu-Forson, J. J. \& Opabonde, J. T. (2005). Review. Seed constraint to cultivation and productivity of African Indigenous Leaf Vegetables. African Journal of Biotechnology. 4 (13): 1480-1484.

Ambrose-Oji, B. (2009). Urban Food Systems and Traditional Leafy Vegetables: Defining the Spaces and Places for Traditional Leafy Vegetables in Urban and Peri-Urban Agriculture. In: Traditional Leafy Vegetables in urban agriculture. (Shackleton C.M., Pasquini M.W., Drescher A.W., eds.) London, Earthscan, 1-33.

Anjorin, S. T. \& Mohammed, M. (2014). Effect of seed-borne fungi on germination and seedling vigour of watermelon (Citrullus lanatus thumb). African Journal of Plant Science. 8 (5): 232-236.

Barak, J. D. \& Liang, A. S. (2008). Role of soil, crop debris and a plant pathogen in Salmonella enterica contamination of tomato plants. PLOS ONE. 3 (2) e1657: 1-5.

Biemond, P. C., Stomph, T. J., Kamara, A., Abdoulaye, T., Herane, S. \& Struik, P. C. (2012). Are investments in an informal seed system for cowpeas a worthwhile endeavour? International Journal of Plant Production. 6 (3): 367- 386.

Bradbury, J. F. (1986). Guide to Plant Pathogenic Bacteria. CAB International Mycological Institute, Wallingford, UK. 1-332.

Broadbent, P., Baker, K. F., Franks, N. \& Holland, J. (1977). Effect of Bacillus spp. on increased growth of seedlings in steamed and in non-treated soil. Phytopathology. 67: 10271034.

Cernansky, R. (2015). Super Vegetables Long Overlooked in Parts of Africa, Indigenous Greens Are Now Capturing Attention for their Nutritional and Environmental Benefits. Nature. 522: 146-148.

Copeland, L. O. \& McDonald, M. B. (2001). Seed Pathology and Pathological Testing. Chapter 16. Book Principles of Seed Science and Technology Publisher Springer US: 354-379. 
Cother, E. J. \& Sivasithamparam, K. (1 $\left.9 \begin{array}{lll}1 & 9 & 3\end{array}\right)$ ). Erwinia: The ottcarora” group, p.8 7106. In Fahy, F.C. and G. J. Presley (eds.), Plant bacterial diseases, a diagnostic guide. Academic Press, Sydney.

Dube, P \& Mujaju, C. (2013). Determination of standards for purity and germination for African Indigenous vegetable (AIV), Blackjack (Bidens pilosa). Advanced Journal of Agricultural Research. 1 (003): 032-038.

Djébali, N., Scott, J. K., Jourdan, M. \& Souissi, T. (2009). Fungi pathogenic on wild radish (Raphanus raphanistrum L.) in northern Tunisia as potential biocontrol agents. Phytopathologia Mediterranea Journal. 48: 205-213.

Guei, R. G., Barra, A. \& Silue, D. (2011). Promoting smallholder seed enterprises: quality seed production of rice, maize, sorghum and millet in northern Cameroon. International Journal of Agricultural Sustainability. 9 (1): 91-99.

Grubben, G. J. H. \& Denton, O. A. (Editors), (2004). Plant Resources of Tropical Africa 2. Vegetables. PROTA Foundation, Wageningen, Netherlands / Backhuys Publishers, Leiden, Netherlands / CTA, Wageningen, Netherlands. 1-668.

Handiseni, M., Sibiya, J., Ogunlela, V. \& Koomen, I. (2008). Comparative study of the effect of different weed management strategies on disease severity and marketable yield of paprika [Capsicum annuum L.] in the smallholder farming sector of Zimbabwe. Journal of Plant Protection Research. 48 (1): 107-117.

Harman, G. E., Howell, C. R., Viterbo, A., Chet, I. \& Lorito, M. (2004). Trichoderma species Opportunistic, avirulent. Plant symbionts. Nature Reviews Microbiology. 2:43-56.

Imbamba, S. K., Ndawula-Senyimba, M. S. \& Papa, G. (1977). The effect of soil moisture stress on photosynthesis transpiration and leaf enlargement in some Kenyan vegetable plants. East African Agricultural and Forestry Journal. 42 (3):309-315.

International Seed Testing Association (ISTA) International Rules for Seed Testing - Seed Health (2015). Seed Science and Technology. 13: 299-355.

International Seed Testing Association (ISTA) International Rules for Seed (2016). Seed Science and Technology. 13: 299-355.

Jager, K. J., Zoccali, C., Kramar, R. \& Dekker, F. W. (2007). Measuring disease occurrence. Kidney International Journal. 72: 412-415. 
Jones, J. B., Pohronezny, K. L., Stall, R. E., \& Jones, J. P. (1986). Survival of Xanthomonas campestris pv. vesicatoria in Florida on tomato crop residue, weeds, seeds, and volunteer tomato plants. Phytopathology. 76: 430-434.

Kuan, T. L. (1988). Inoculum thresholds of seed-borne pathogens. The American Phytopathological Society. Overview. 78 (6): 867-868.

Mancini, V. \& Romanazzi, G. (2013). Seed treatments to control seedborne fungal pathogens of vegetable crops. Pest Management Science. 70: 860-868.

Mathur, S. B. \& Olga, K. (2001). Common Laboratory Seed Health Testing Methods of Detecting Fungi. Danish Government Institute of Seed Pathology for Developing countries. Kandrups Bogtrigkkeri, $1^{\text {st }}$ edition. 1-413.

Marthur, R. L. \& Sehgal, S. P. (1964). Fungal mycoflora of seeds of jowar (Sorghum vulgare), Its role in reduced emergence and vigour of seedling and control. Indian Journal of Phytopathology. 17: 227-233.

Mukwereza, L. ( $\left.\begin{array}{llll}2 & 0 & 0 & 2\end{array}\right)$. Report on the Study on Processed Indigenous Vegetables (IV's): Mutare city and its Peri-urban areas. A study undertaken as part of activities for the CPHPfunded IV's project. 1-16.

Naseri, B. \& Emami, H. (2013). Impact of bean growing region n root rot pathogens infecting root tissues, seed and field soil. In: Proceedings of the $6^{\text {th }}$ Congress of Agricultural Research Findings. Sanandaj: Sanandai University.

Naseri, B. \& Mousavi, S. S. (2015). Root rot pathogens in field soil, roots and seeds in relation to common bean (Phaseolus vulgaris), disease and seed production. International Journal of Pest Management. 61 (1): 60-67.

Ngadze, E. (2014). In vitro and greenhouse evaluation of botanical extracts for antifungal activity against Phytophthora infestans. Journal of Biopesticides. 7 (2): 198-203.

Ngadze, E. \& Icishahayo, D. (2014). Survey: assess the distribution and impact of potato blackleg and soft rot diseases in Zimbabwe. Journal of Agriculture and Veterinary Science. 7 (2): 126-132.

Ngugi, I. K., Gitau, R. \& Nyoro, J. K. (2007). Access to high value markets by smallholder farmers of African Indigenous Vegetables in Kenya. Regoverning Markets Innovative Practice series, IIED, London. Sustainable Markets Group: 1-28. 
Ngwerume, F. C. and Mvere, B. (2003). Report on the findings of a socio-economic survey on the marketing, consumption and production of traditional vegetables in the urban and peri urban areas of Harare, Zimbabwe. DR \& SS-HRC/NRI project a0892.

Rothwell, A. (1982). A revised list of Plant Diseases occurring in Zimbabwe. Journal of the Federal Herbarium, Salisbury, Rhodesia and Nyasaland. 12 (2): 233-351.

Rude, S. V., Duczek, L. J. \& Seidle, E. (1999). The effect of Alternaria brassicae, Alternaria raphani and Alternaria alternata on seed germination of Brassica rapa canola. Seed Science and Technology. 27: 795-798.

Russell, W. \& Herwald, H. (2005). Concepts in Bacterial Virulence. Contribution Microbiology. Basel. Karger. 12: 55-66.

Schaad, N. W., Jones, J. B. \& Chun, W. eds (2001). Laboratory Guide for identification of Plant Pathogenic Bacteria. Third edition. The American Phytopathological Society. 1-373.

Schippers, R. R. (2000). African Indigenous Vegetables. An overview of the cultivated species. Chatham, UK: Natural Resources Institute/ACP-EU Technical Centre of Agricultural and Rural Cooperation Book: 1-210.

Sharma, A. D., Tnakur, V. \& Munjal, R. L. (1980). Seed myeoflora of Amaranthus eaudatus, its pathology and control. Indian Phytopathology. 33: 242-244.

Valkonen, J. P. T. \& Koponen, H. (1990). Seed-borne fungi of Chinese cabbage (Brassica pekinensis), their pathogenicity and control. Plant Pathology. 39: 510-516.

Vincent, V. \& Thomas, R.G. (1960). An agro-ecological Survey of Southern Rhodesia Part 1: Agro-ecological Survey: Government Printers. Salisbury. 1-217.

Walcott, R. R. (2003). Detection of seedborne pathogens. HortTechnology. 13 (1): 40-47.

Weinberger, K. \& Msuya, J. (2004). Indigenous Vegetables in Tanzania-Significance and Prospects. Shanhua, Taiwan: AVRDC-The World Vegetable Center, Technical Bulletin No. 31, AVRDC Publication 04-600. 1-70.

https://www.worldweatheronline.com Historical average weather 20/04/2017 


\section{Annex}

\section{Annex 1 Climatic data during crop growth of the origin sites of the seed}

\begin{tabular}{|c|c|c|c|c|c|c|c|}
\hline \multirow[t]{2}{*}{ Weather } & Dec & Jan & Feb & March & April & May & June \\
\hline & \multicolumn{7}{|c|}{ Farmer 1} \\
\hline Temperature $\left({ }^{\circ} \mathrm{C}\right)$ & 31 & 29 & 30 & 28 & 26 & 23 & 22 \\
\hline Rain (mm) & 73 & 56 & 49 & 98 & 68.5 & 11.2 & 0.3 \\
\hline$\%$ Cloud cover & 24 & 36 & 27 & 33 & 25 & 17 & 5 \\
\hline$\%$ Humidity & 57 & 69 & 72 & 81 & 68 & 61 & 56 \\
\hline Bee activity & - & - & mod & $\bmod$ & high & low & - \\
\hline \multirow[t]{2}{*}{ Planting } & \multicolumn{7}{|c|}{ Sole crop } \\
\hline & \multicolumn{7}{|c|}{ Farmer 2} \\
\hline Temperature $\left({ }^{\circ} \mathrm{C}\right)$ & 31 & 29 & 30 & 28 & 26 & 22 & 21 \\
\hline Rain (mm) & 100 & 49 & 46 & 85 & 29.5 & 17 & 0.8 \\
\hline$\%$ Cloud cover & - & 39 & 33 & 38 & 22 & 20 & 13 \\
\hline$\%$ Humidity & - & 67 & 76 & 79 & 69 & 67 & 63 \\
\hline Bee activity & - & - & - & $\bmod$ & high & low & - \\
\hline \multirow[t]{2}{*}{ Planting } & \multicolumn{7}{|c|}{ Intercropped with maize } \\
\hline & \multicolumn{7}{|c|}{ Farmer 3} \\
\hline Temperature $\left({ }^{\circ} \mathrm{C}\right)$ & 31 & 29 & 30 & 28 & 26 & 22 & 21 \\
\hline Rain (mm) & 100 & 49 & 46 & 85 & 29.5 & 17 & 0.8 \\
\hline$\%$ Cloud cover & - & 39 & 33 & 38 & 22 & 20 & 13 \\
\hline$\%$ Humidity & - & 67 & 76 & 79 & 69 & 67 & 63 \\
\hline Bee activity & - & - & $\bmod$ & $\bmod$ & high & low & - \\
\hline \multirow[t]{2}{*}{ Planting } & \multicolumn{7}{|c|}{ Intercropped with maize } \\
\hline & \multicolumn{7}{|c|}{ Farmer 4} \\
\hline Temperature $\left({ }^{\circ} \mathrm{C}\right)$ & 31 & 29 & 30 & 28 & 26 & 22 & 21 \\
\hline Rain (mm) & 110 & 57 & 63 & 29 & 15 & 10 & 1.5 \\
\hline$\%$ Cloud cover & - & 39 & 33 & 38 & 22 & 20 & 13 \\
\hline$\%$ Humidity & - & 67 & 76 & 79 & 69 & 67 & 63 \\
\hline Bee activity & - & - & mod & $\bmod$ & high & - & - \\
\hline \multirow[t]{2}{*}{ Planting } & \multicolumn{7}{|c|}{ Sole crop } \\
\hline & \multicolumn{7}{|c|}{ Farmer 5} \\
\hline Temperature $\left({ }^{\circ} \mathrm{C}\right)$ & 29 & 27 & 28 & 27 & - & - & - \\
\hline Rain (mm) & 105.7 & 14.5 & 16.7 & 24.8 & - & - & - \\
\hline$\%$ Cloud cover & 24 & 33 & 30 & 36 & 29 & 21 & 17 \\
\hline
\end{tabular}




\begin{tabular}{lccccccc} 
\% Humidity & 57 & 68 & 67 & 77 & 70 & 69 & 64 \\
Bee activity & - & - & - & - & - & - & - \\
Planting & & \multicolumn{8}{c}{ Sole crop }
\end{tabular}

a. https://www.worldweatheronline.com Historical average weather 20/04/2017

b. authors

Annex 2 Geographical coordinates and climatic data during crop growth of the origin sites of the seed

The seed was sourced from three different agro ecological regions of Zimbabwe: Mashonaland Central, Mashonaland West and Mashonaland East. The five seed sources were Horticultural Research Centre [HRC] (Farmer 5), Shamva (Farmer 4), Murehwa - two farmers (Macheya- Farmer 2 and Makondora- Farmer 3) and Chinhoyi (Farmer 1). HRC is a government institute located in Mashonaland East province and is under agro ecological region $2 \mathrm{~B}\left(18^{\circ} 11^{\prime} 6.97^{\prime \prime} \mathrm{S}\right.$ : $\left.31^{\circ} 33^{\prime} 6.95^{\prime \prime} \mathrm{E}\right)$ at an elevation of $1688 \mathrm{~m}$ above sea level (Vincent \& Thomas, 1960). The mean annual rainfall for the area ranges from $850-900 \mathrm{~mm}$. The mean annual temperature ranges from $18-29^{\circ} \mathrm{C}$. During seed crop growth temperatures and rainfall ranged (December 2015- March 2016) from 28.9-26.7 ${ }^{\circ} \mathrm{C}$ and from 105.7-24.8 mm, respectively. Shamva is in Mashonaland Central province and located under agro ecological region $3\left(17^{\circ} 18^{\prime} 56^{\prime \prime} \mathrm{S}: 31^{\circ} 34^{\prime} 14^{\prime \prime} \mathrm{E}\right)$ at an elevation of $953 \mathrm{~m}$ above sea level, receiving annual rainfall of 800-850 mm (Vincent \& Thomas, 1960). The mean temperature for Shamva ranges between $21-31^{\circ} \mathrm{C}$. During seed crop growth temperatures and rainfall ranged (December 2015- June 2016) from $31-21^{\circ} \mathrm{C}$ and from $110-1.5 \mathrm{~mm}$, respectively. Murehwa is located in Mashonaland East province under agro ecological region 3 (17 $38^{\prime} 35.59 " \mathrm{~S}: 31^{\circ} 47^{\prime} 2.40^{\prime \prime} \mathrm{E}$ ) at an elevation of $1400 \mathrm{~m}$ above sea level (Vincent \& Thomas, 1960). The mean annual rainfall ranges from $800-850 \mathrm{~mm}$. The mean annual temperature ranges from $18-29^{\circ} \mathrm{C}$. During seed crop growth temperatures and rainfall were (December 2015- June 2016) $31-21^{\circ} \mathrm{C}$ and 100 $0.8 \mathrm{~mm}$, respectively. Chinhoyi is located in Mashonaland West province under agro

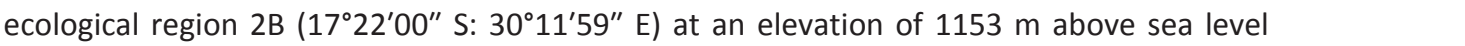
(Vincent \& Thomas, 1960). The mean annual rainfall and temperature ranges from 700-800 $\mathrm{mm}$ and $22-29^{\circ} \mathrm{C}$, respectively. During seed crop growth temperatures and rainfall ranged (December 2015- June 2016) $31-22^{\circ} \mathrm{C}$ and $73-0.31 \mathrm{~mm}$, respectively, (authors, 
https://www.worldweatheronline.com Historical average weather 20/04/2017), data presented as Annex 1. 
Annex 3 to 12, the mean separation indicates per pathogen the differences among the interactions between farmers and three species (Duncan's multiple range least significant difference test, $P \leq 0$.0).SFor all cases, all values in parentheses are transformed values and mean separation.

Annex 3 The two-way interactions between the farmers and plant species percentage occurrence of Xanthomonas campestris

$\begin{array}{rr}\text { Farmer*Species } & \text { Mean } \\ \text { Farmer 2 Amaranthus hybridus } & 0.0(0.0 \mathrm{a}) \\ \text { Farmer 3 Amaranthus hybridus } & 0.0(0.0 \mathrm{a}) \\ \text { Farmer 4 Amaranthus hybridus } & 0.0(0.0 \mathrm{a}) \\ \text { Farmer 5 Amaranthus hybridus } & 0.0(0.0 \mathrm{a}) \\ \text { Farmer 4 Bidens pilosa } & 0.0(0.0 \mathrm{a}) \\ \text { Farmer 5 Bidens pilosa } & 0.0(0.0 \mathrm{a}) \\ \text { Farmer 1 Cleome gynandra } & 0.0(0.0 \mathrm{a}) \\ \text { Farmer 1 Amaranthus hybridus } & 0.67(0.44 \mathrm{~b}) \\ \text { Farmer 2 Bidens pilosa } & 9.33(1.97 \mathrm{c}) \\ \text { Farmer 5 Cleome gynandra } & 9.67(1.99 \mathrm{c}) \\ \text { Farmer 1 Bidens pilosa } & 10.0(2.00 \mathrm{c}) \\ \text { Farmer 2 Cleome gynandra } & 10.0(2.00 \mathrm{c}) \\ \text { Farmer 3 Cleome gynandra } & 10.0(2.00 \mathrm{c}) \\ \text { Farmer 3 Bidens pilosa } & 10.0(2.00 \mathrm{c}) \\ \text { Farmer 4 Cleome gynandra } & 10.0(2.00 \mathrm{c}) \\ \text { LSD 5\% } & \text { 0.33 }\end{array}$


Annex 4 The two-way interactions between the farmers and plant species percentage occurrence of Pseudomonas syringae

Farmer*Species

Farmer 2 Amaranthus hybridus

Farmer 1 Bidens pilosa

Farmer 1 Cleome gynandra

Farmer 2 Cleome gynandra

Farmer 3 Bidens pilosa

Farmer 3 Cleome gynandra

Farmer 4 Amaranthus hybridus

Farmer 4 Cleome gynandra

Farmer 5 Amaranthus hybridus

Farmer 5 Cleome gynandra

Farmer 2 Bidens pilosa

Farmer 1 Amaranthus hybridus

Farmer 4 Bidens pilosa

Farmer 5 Bidens pilosa

Farmer 3 Amaranthus hybridus

LSD 5\%
Mean

0.0 (0.0a)

$0.0(0.0 \mathrm{a})$

0.0 (0.0a)

0.0 (0.0a)

$0.0(0.0 a)$

$0.0(0.0 \mathrm{a})$

0.0 (0.0a)

0.0 (0.0a)

0.0 (0.0a)

0.0 (0.0a)

0.67 (0.44ab)

1.0 (0.50ab)

2.0 (0.99bc)

1.67 (1.23c)

2.33 (1.33c)

0.63 
Annex 5 The two-way interactions between the farmers and plant species percentage occurrence of Bacillus spp.

$\begin{array}{rr}\text { Farmer*Species } & \text { Mean } \\ \text { Farmer 1 Bidens pilosa } & 0.0(0.0 \mathrm{a}) \\ \text { Farmer 1 Cleome gynandra } & 0.0(0.0 \mathrm{a}) \\ \text { Farmer 2 Bidens pilosa } & 0.0(0.0 \mathrm{a}) \\ \text { Farmer 2 Cleome gynandra } & 0.0(0.0 \mathrm{a}) \\ \text { Farmer 3 Bidens pilosa } & 0.0(0.0 \mathrm{a}) \\ \text { Farmer 3 Cleome gynandra } & 0.0(0.0 \mathrm{a}) \\ \text { Farmer 4 Bidens pilosa } & 0.0(0.0 \mathrm{a}) \\ \text { Farmer 4 Cleome gynandra } & 0.0(0.0 \mathrm{a}) \\ \text { Farmer 5 Bidens pilosa } & 0.0(0.0 \mathrm{a}) \\ \text { Farmer 5 Cleome gynandra } & 0.0(0.0 \mathrm{a}) \\ \text { Farmer 1 Amaranthus hybridus } & 0.0(0.0 \mathrm{a}) \\ \text { Farmer 2 Amaranthus hybridus } & 0.0(0.0 \mathrm{a}) \\ \text { Farmer 3 Amaranthus hybridus } & 0.0(0.0 \mathrm{a}) \\ \text { Farmer 4 Amaranthus hybridus } & 0.0(0.0 \mathrm{a}) \\ \text { Farmer 5 Amaranthus hybridus } & 0.33(0.35 \mathrm{~b}) \\ \text { LSD 5\% } & \mathbf{0 . 2 6}\end{array}$


Annex 6 The two-way interactions between the farmers and plant species percentage occurrence of Alternaria alternata

\begin{tabular}{rr} 
Farmer*Species & Mean \\
Farmer 1 Bidens pilosa & $0.0(0.0 \mathrm{a})$ \\
Farmer 2 Amaranthus hybridus & $0.0(0.0 \mathrm{a})$ \\
Farmer 3 Amaranthus hybridus & $0.0(0.0 \mathrm{a})$ \\
Farmer 4 Amaranthus hybridus & $0.0(0.0 \mathrm{a})$ \\
Farmer 5 Amaranthus hybridus & $0.0(0.0 \mathrm{a})$ \\
Farmer 5 Cleome gynandra & $0.33(0.35 \mathrm{ab})$ \\
Farmer 4 Bidens pilosa & $0.33(0.35 \mathrm{ab})$ \\
Farmer 1 Amaranthus hybridus & $0.67(0.69 \mathrm{bc})$ \\
Farmer 3 Bidens pilosa & $0.67(0.69 \mathrm{bc})$ \\
Farmer 1 Cleome gynandra & $1.67(1.23 \mathrm{~cd})$ \\
Farmer 5 Bidens pilosa & $1.67(1.23 \mathrm{~cd})$ \\
Farmer 3 Cleome gynandra & $2.33(1.33 \mathrm{~d})$ \\
Farmer 2 Cleome gynandra & $2.33(1.33 \mathrm{~d})$ \\
Farmer 4 Cleome gynandra & $2.67(1.38 \mathrm{~d})$ \\
Farmer 2 Bidens pilosa & $2.67(1.44 \mathrm{~d})$ \\
\hline LSD 5\% & 0.57
\end{tabular}


Annex 7 The two-way interactions between the farmers and plant species percentage occurrence of Aspergillus spp.

Farmer*Species

Farmer 5 Amaranthus hybridus

Farmer 1 Amaranthus hybridus

Farmer 1 Bidens pilosa

Farmer 1 Cleome gynandra

Farmer 2 Amaranthus hybridus

Farmer 2 Bidens pilosa

Farmer 2 Cleome gynandra

Farmer 3 Amaranthus hybridus

Farmer 3 Bidens pilosa

Farmer 3 Cleome gynandra

Farmer 4 Amaranthus hybridus

Farmer 4 Cleome gynandra

Farmer 5 Cleome gynandra

Farmer 5 Bidens pilosa

Farmer 4 Bidens pilosa

LSD 5\%
Mean

0.0 (0.0a)

0.0 (0.0a)

0.0 (0.0a)

$0.0(0.0 \mathrm{a})$

0.0 (0.0a)

$0.0(0.0 \mathrm{a})$

0.0 (0.0a)

0.0 (0.0a)

0.0 (0.0a)

0.0 (0.0a)

0.0 (0.0a)

0.0 (0.0a)

0.0 (0.0a)

0.33 (0.35ab)

2.0 (0.60b)

0.51 
Annex 8 The two-way interactions between the farmers and plant species percentage occurrence of Fusarium oxysporum

$\begin{array}{rr}\text { Farmer*Species } & \text { Mean } \\ \text { Farmer 1 Bidens pilosa } & 0.0(0.0 \mathrm{a}) \\ \text { Farmer 1 Cleome gynandra } & 0.0(0.0 \mathrm{a}) \\ \text { Farmer 2 Amaranthus hybridus } & 0.0(0.0 \mathrm{a}) \\ \text { Farmer 2 Cleome gynandra } & 0.0(0.0 \mathrm{a}) \\ \text { Farmer 3 Amaranthus hybridus } & 0.0(0.0 \mathrm{a}) \\ \text { Farmer 3 Bidens pilosa } & 0.0(0.0 \mathrm{a}) \\ \text { Farmer 3 Cleome gynandra } & 0.0(0.0 \mathrm{a}) \\ \text { Farmer 4 Bidens pilosa } & 0.0(0.0 \mathrm{a}) \\ \text { Farmer 5 Bidens pilosa } & 0.0(0.0 \mathrm{a}) \\ \text { Farmer 5 Cleome gynandra } & 0.0(0.0 \mathrm{a}) \\ \text { Farmer 4 Amaranthus hybridus } & 0.33(0.35 \mathrm{ab}) \\ \text { Farmer 2 Bidens pilosa } & 0.67(0.69 \mathrm{bc}) \\ \text { Farmer 4 Cleome gynandra } & 1.67(0.88 \mathrm{bc}) \\ \text { Farmer 1 Amaranthus hybridus } & 3.33(1.14 \mathrm{c}) \\ \text { Farmer 5 Amaranthus hybridus } & 2.33(1.34 \mathrm{c}) \\ \text { LSD 5\% } & \mathbf{0 . 6 7}\end{array}$


Annex 9 The two-way interactions between the farmers and plant species percentage occurrence of Curvularia spp.

Farmer*Species

Farmer 3 Amaranthus hybridus

Farmer 5 Amaranthus hybridus

Farmer 2 Amaranthus hybridus

Farmer 2 Cleome gynandra

Farmer 4 Bidens pilosa

Farmer 5 Bidens pilosa

Farmer 5 Cleome gynandra

Farmer 4 Cleome gynandra

Farmer 1 Amaranthus hybridus

Farmer 3 Bidens pilosa

Farmer 4 Amaranthus hybridus

Farmer 2 Bidens pilosa

Farmer 1 Cleome gynandra

Farmer 1 Bidens pilosa

Farmer 3 Cleome gynandra

LSD 5\%
Mean

0.0 (0.0a)

0.0 (0.0a)

0.0 (0.0a)

$0.0(0.0 \mathrm{a})$

0.0 (0.0a)

0.0 (0.0a)

0.0 (0.0a)

$0.33(0.35 a b)$

0.67 (0.44ab)

1.67 (0.57ab)

0.67 (0.69ab)

1.0 (1.04b)

$5.0(1.70 \mathrm{c})$

6.67 (1.83c)

7.33 (1.87c)

0.65 
Annex 10 The two-way interactions between the farmers and plant species percentage occurrence of Phoma betae

$\begin{array}{rr}\text { Farmer*Species } & \text { Mean } \\ \text { Farmer 1 Bidens pilosa } & 0.0(0.0 \mathrm{a}) \\ \text { Farmer 2 Bidens pilosa } & 0.0(0.0 \mathrm{a}) \\ \text { Farmer 3 Bidens pilosa } & 0.0(0.0 \mathrm{a}) \\ \text { Farmer 4 Amaranthus hybridus } & 0.0(0.0 \mathrm{a}) \\ \text { Farmer 4 Cleome gynandra } & 0.0(0.0 \mathrm{a}) \\ \text { Farmer 5 Bidens pilosa } & 0.0(0.0 \mathrm{a}) \\ \text { Farmer 1 Amaranthus hybridus } & 0.0(0.0 \mathrm{a}) \\ \text { Farmer 1 Cleome gynandra } & 0.0(0.0 \mathrm{a}) \\ \text { Farmer 2 Amaranthus hybridus } & 0.0(0.0 \mathrm{a}) \\ \text { Farmer 2 Cleome gynandra } & 0.0(0.0 \mathrm{a}) \\ \text { Farmer 3 Amaranthus hybridus } & 0.0(0.0 \mathrm{a}) \\ \text { Farmer 3 Cleome gynandra } & 0.0(0.0 \mathrm{a}) \\ \text { Farmer 5 Amaranthus hybridus } & 0.0(0.0 \mathrm{a}) \\ \text { Farmer 5 Cleome gynandra } & 0.0(0.0 \mathrm{a}) \\ \text { Farmer 4 Bidens pilosa } & 0.67(0.44 \mathrm{~b}) \\ \text { LSD 5\% } & \mathbf{0 . 3 3}\end{array}$


Annex 11 The two-way interactions between the farmers and plant species percentage occurrence of Colletotrichum spp.

Farmer*Species

Farmer 1 Amaranthus hybridus Farmer 1 Bidens pilosa

Farmer 1 Cleome gynandra

Farmer 2 Amaranthus hybridus

Farmer 2 Bidens pilosa

Farmer 3 Amaranthus hybridus

Farmer 3 Bidens pilosa

Farmer 3 Cleome gynandra

Farmer 4 Amaranthus hybridus

Farmer 4 Bidens pilosa

Farmer 4 Cleome gynandra

Farmer 5 Amaranthus hybridus

Farmer 5 Bidens pilosa

Farmer 5 Cleome gynandra

Farmer 2 Cleome gynandra

LSD 5\%
Mean

0.0 (0.0a)

0.0 (0.0a)

0.0 (0.0a)

$0.0(0.0 \mathrm{a})$

0.0 (0.0a)

$0.0(0.0 \mathrm{a})$

0.0 (0.0a)

0.0 (0.0a)

0.0 (0.0a)

0.0 (0.0a)

0.0 (0.0a)

0.0 (0.0a)

0.0 (0.0a)

0.0 (0.0a)

0.67 (0.69b)

0.26 
Annex 12 The two-way interactions between the farmers and plant species percentage occurrence of Rhizoctonia solani

$\begin{array}{rr}\text { Farmer*Species } & \text { Mean } \\ \text { Farmer 1 Bidens pilosa } & 0.0(0.0 \mathrm{a}) \\ \text { Farmer 1 Cleome gynandra } & 0.0(0.0 \mathrm{a}) \\ \text { Farmer 2 Amaranthus hybridus } & 0.0(0.0 \mathrm{a}) \\ \text { Farmer 2 Bidens pilosa } & 0.0(0.0 \mathrm{a}) \\ \text { Farmer 2 Cleome gynandra } & 0.0(0.0 \mathrm{a}) \\ \text { Farmer 3 Amaranthus hybridus } & 0.0(0.0 \mathrm{a}) \\ \text { Farmer 3 Bidens pilosa } & 0.0(0.0 \mathrm{a}) \\ \text { Farmer 3 Cleome gynandra } & 0.0(0.0 \mathrm{a}) \\ \text { Farmer 4 Amaranthus hybridus } & 0.0(0.0 \mathrm{a}) \\ \text { Farmer 4 Bidens pilosa } & 0.0(0.0 \mathrm{a}) \\ \text { Farmer 4 Cleome gynandra } & 0.0(0.0 \mathrm{a}) \\ \text { Farmer 5 Amaranthus hybridus } & 0.0(0.0 \mathrm{a}) \\ \text { Farmer 5 Bidens pilosa } & 0.0(0.0 \mathrm{a}) \\ \text { Farmer 5 Cleome gynandra } & 0.0(0.0 \mathrm{a}) \\ \text { Farmer 1 Amaranthus hybridus } & 0.67(0.44 \mathrm{~b}) \\ & 0.33\end{array}$


3 Seed germination of traditional leafy vegetables ${ }^{2}$

2 Paper by Praxedis Dube and Paul C. Struik, 2018. Seed germination of traditional leafy vegetables. African Journal of Agricultural Research 


\section{Abstract}

Traditional leafy vegetables provide affordable sources of micronutrients to many people in Zimbabwe. They are produced by smallholder farmers with limited knowledge on the agronomic value of high-quality seed and the seed is rarely tested to determine its quality. This study was therefore conducted to evaluate the germination of seeds of three traditional leafy vegetables sourced from five different farmers and the morphological characteristics of the seedlings. The seeds were collected in the growing season of 20152016, stored at room temperature and tested for germination in the laboratory at regular monthly intervals during storage. Field germination tests were conducted only once. The seeds tested were classified as normal seedlings, abnormal seedlings, fresh nongerminated seeds or dead seeds. Morphological analysis was conducted at seedling stage, including seven qualitative characters and one quantitative character. Mean separation based on the least significance difference at $5 \%$ indicated that percentage germination, purity and 1000-seeds weight for all three species significantly differed among sources. The results showed that Cleome gynandra had lowest germination percentages, Amaranthus hybridus was intermediate and Bidens pilosa had highest values throughout the tests. Cleome gynandra seed exhibited dormancy, showing high levels of fresh nongerminated seed percentage after each test, which is potentially disastrous for the farmers. The combination of pre-chilling, light, potassium nitrate and alternating temperatures as a way of improving germination for Cleome gynandra needs further validation. 


\subsection{Introduction}

The term traditional leafy vegetables (TLVs) of Africa refers to plant species which originate on the continent and have a long history of cultivation, domestication and use in African conditions (Ambrose-Oji, 2009). TLVs' cultivation, domestication and use are tremendous

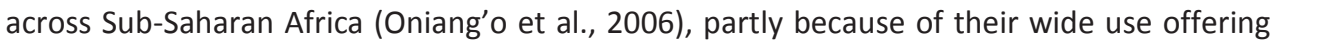
important socio-economic benefits. TLVs' consumption and marketing across Africa have increased steadily with the growing demand from consumers as well (Ojiewo et. al., 2012). For example, in Zimbabwe more than $75 \%$ of the population eat them when available (DFID project 2309, 2003); most commonly consumed are: spider flower (Cleome gynandra), pumpkin leaves (Cucurbita spp.), pigweed (Amaranthus spp.), cowpea leaves (Vigna unguiculata), black jack (Bidens pilosa), okra (Abelmoschus esculentus) and jute leaves (Corchorus olitorius).

The production of TLVs is challenged by many factors including unavailability of quality seeds (Abukutsa-Onyango, 2007), low germination rates of seed (Cernansky, 2015), the problems of purity (Karanja et al., 2012), limited knowledge on the agronomic value of high-quality seed among smallholder farmers (Biemond et al., 2012) and poor seed production skills by smallholder farmers as they rely on traditional seed production technologies to produce their seed (Schippers, 2000). The seeds are rarely tested for viability before they are planted (Schippers, 2000) giving them less economic value on national markets. The seed markets remain localised. Moreover, the farmers get very low profits as they fail to fetch premium prices on both vegetables and seed markets, because the seeds have not gone through any form of certification or quality check before being marketed, and lack quality declaration documentation such as seed testing certificates, which are required for regional and international seed trade (Cromwell et al., 1992). When planting the seeds, the farmers are at times forced to use large quantities of seed to compensate for the poor germination rates and this becomes less economically viable. Furthermore, at harvesting, because the variety admixtures emanating from the use of less pure seed, the farmers tend to selectively harvest, which is cumbersome and time consuming. All these challenges have constrained the ability of farmers to meet the demand and to deliver quality produce to consumers (Afari-Sefa et al., 2013). 
Of the total TLV seed requirements across Sub Saharan Africa, only a tenth is of good quality and available to the farmers through formal seed markets and the remainder is met by the farmers themselves as saved seed or seed obtained through exchange (Ngwerume \& Mvere, 2003; Adebooye et al., 2005). Notably, over three quarters of the farmers that venture into TLVs production use saved seed for planting their crops in Zimbabwe (Schippers, 2000) and its quality is rarely known. Farmers using poor-quality seed risk poor germination and poor crop stand causing low production and productivity, which directly affects their yields (Karanja et al., 2012) and the quality (Ayana et al., 2014) of their produce, and therefore reduces their profits. This can be addressed by regular checks on the quality of the seed, getting the required quality declaration documentation before the seeds are distributed or sold. Such evaluations are done in the laboratory under optimal conditions. In some cases, skilled farmers do regular checks on quality of their seeds before planting. The commonly used method by these farmers is testing germination by putting just a few seeds in the soil.

Seed germination and purity analysis are the most important components that can be used to differentiate the quality of the seed among farmers, and can be used to accord those that produce good-quality seed. It is also equally important to determine the germination so as best seed rates are known before the seeds are sown. Seed germination and purity tests have been done on many agricultural crops, trees, flowers, and herbs for the international, regional and local markets in Zimbabwe (Seeds Rules and Regulations, 1971) and (International Seed Testing Association [ISTA], 2016). Very few studies that determine the quality of traditional leafy vegetable seed have been done in Zimbabwe. Notably, the Seeds Rules and Regulations (1971) of Zimbabwe does not have guidelines for seed certification for these plant species, as they are only regarded as weeds (Seed Act (1971)) and they are not covered in many seed regulatory platforms (Dube \& Mujaju, 2013). This justifies the adoption of this study on farmers saved seed which aimed at 1) evaluating the germination of traditional leafy vegetable seeds and 2) determining the morphological characters of seedlings from five sources. 
Therefore the hypotheses tested include:

- Percentage germination varies among seed sources.

- Qualitative and quantitative morphological characteristics vary by seed sources. 


\subsection{Methodology and Data Analysis}

The study uses three traditional leafy vegetable species, namely Cleome gynandra, Bidens pilosa and Amaranthus hybridus as examples. These three species were selected because they are one of the commonly consumed traditional leafy vegetables in Zimbabwe. Knowing their planting value before planting can increase the farmers' incomes as they are able to predict desired crop stand, reduce labour cost for gap filling or thinning crops.

\section{Origin of the seed sources}

Seeds of three species were sourced from four farmers and a research organization. These seed sources were chosen using a snow ball sampling technique, with as entry point the traditional leafy vegetable markets. Only farmers who had seed stocks from the previous season showing that they had been producing TLV seed were chosen. The research organization was included for comparison purposes since most of the seed is produced by smallholder farmers with limited knowledge, as alluded earlier.

During the seed development time, factors that might influence seed quality including weather variation were also recorded weekly (see Annex 1 for full results). About 200 grams of seed per species was sourced from each farmer to be used for germination tests and morphological characterisation. The seed collection constituted of 15 samples (samples of three species per seed source). The seed was stored at room temperature and seed germination in the laboratory was analysed at regular time intervals during storage. The germination tests were conducted from August to November 2016 and in February, 2017. The laboratory tests were conducted at the Seed Services in the Department of Research and Specialist Services under the Ministry of Agriculture, Mechanisation and Irrigation Development. Field germination tests were conducted in December, 2016, only once.

The seed was sourced from three different agro ecological regions of Zimbabwe which entailed: Mashonaland Central, Mashonaland West and Mashonaland East. The five seed sources entailed: Chinhoyi (Farmer 1), Murehwa - two farmers (Farmer 2 and Farmer 3), Shamva (Farmer 4) and research organization (Farmer 5). The climatic conditions during crop growth and geographical coordinates of the seed origin sites are presented in Table 8. 
Table 8 Geographical coordinates and climatic data during crop growth of the origin sites of the seed

\begin{tabular}{|c|c|c|c|c|c|c|c|c|}
\hline Farmer & Origin & $\begin{array}{l}\text { Agro } \\
\text { ecological } \\
\text { region }^{a}\end{array}$ & $\begin{array}{l}\text { Mean annual } \\
\text { temperature }\end{array}$ & $\begin{array}{l}\text { Temperature } \\
\text { range during } \\
\text { crop growth }\end{array}$ & $\begin{array}{l}\text { Geographical } \\
\text { coordinates }\end{array}$ & $\begin{array}{l}\text { Altitude } \\
\text { (m) }\end{array}$ & $\begin{array}{l}\text { Annual } \\
\text { rainfall } \\
(\mathrm{mm})\end{array}$ & $\begin{array}{l}\text { Amount of } \\
\text { rainfall } \\
\text { during crop } \\
\text { growth } \\
(\mathrm{mm})\end{array}$ \\
\hline 1 & $\begin{array}{l}\text { Mashonaland } \\
\text { West }\end{array}$ & $2 B$ & $22-29^{\circ} \mathrm{C}$ & $31-22^{\circ} \mathrm{C}$ & $\begin{array}{lllllllll}1 & 7 & { }^{\circ} 2 & 2 & \prime & 0 & 0 & \prime \prime \\
3 & 0 & \circ & 1 & 1 & 5 & 9 & \prime \prime\end{array}$ & 1153 & $700-800$ & $73-0.31$ \\
\hline 2 & $\begin{array}{l}\text { Mashonaland } \\
\text { East }\end{array}$ & 3 & $18-29^{\circ} \mathrm{C}$ & $31-21^{\circ} \mathrm{C}$ & $\begin{array}{l}17^{\circ} 38^{\prime} 35.59^{\prime \prime} \\
\text { S; } 31^{\circ} 47^{\prime} 2.40^{\prime \prime} \\
\text { E }\end{array}$ & 1400 & $800-850$ & $100-0.8$ \\
\hline 3 & $\begin{array}{l}\text { Mashonaland } \\
\text { East }\end{array}$ & 3 & $18-29^{\circ} \mathrm{C}$ & $31-21^{\circ} \mathrm{C}$ & $\begin{array}{l}17^{\circ} 38^{\prime} 35.59^{\prime \prime} \\
S ; 31^{\circ} 47^{\prime} 2.40^{\prime \prime} \\
\text { E }\end{array}$ & 1400 & $800-850$ & $100-0.8$ \\
\hline 4 & $\begin{array}{l}\text { Mashonaland } \\
\text { Central }\end{array}$ & 3 & $21-31^{\circ} \mathrm{C}$ & $31-21^{\circ} \mathrm{C}$ & $\begin{array}{l}17^{\circ} 18^{\prime} 56^{\prime \prime} \mathrm{S} ; \\
31^{\circ} 34^{\prime} 14^{\prime \prime} \mathrm{E}\end{array}$ & 953 & $800-850$ & $110-1.5$ \\
\hline 5 & $\begin{array}{l}\text { Mashonaland } \\
\text { East }\end{array}$ & $2 \mathrm{~B}$ & $18-29^{\circ} \mathrm{C}$ & $29-27^{\circ} \mathrm{C}$ & $\begin{array}{l}18^{\circ} 11^{\prime} 6.97^{\prime \prime} \mathrm{S} ; \\
\left.31^{\circ} 33^{\prime} 6.95^{\prime \prime} \mathrm{E}\right)\end{array}$ & 1688 & $850-900$ & $105.7-24.8$ \\
\hline
\end{tabular}

a. Note: Agro ecological classification also known as natural region (NR) classification of Zimbabwe, divided the country into five regions based on mean annual rainfall and was done in the 1960s (Vincent \& Thomas, 1960).

b. Source: authors, https://www.worldweatheronline.com Historical average weather 20/04/2017 (for full details see Annex 1 and description)

\section{Laboratory and field experiments}

The seed was sub-sampled to working samples by using the hand-halving method as described by the International Seed Testing Association (ISTA, 2016). Thereafter, the purity analysis was conducted on each working sample. The findings were classified as pure seed, inert matter (comprising broken seed, sand, chaff, stalks) and other seeds. All the purity analyses were done in accordance with the Pure Seed Definition provided for each crop species (ISTA, 2016) and procedures applicable to Tagetes tenuifolia were used for Bidens pilosa. Other seeds were identified with the aid of reference samples (seed of other crop species collected and filed according to ISTA requirements at Seed Services). Thereafter, each component was weighed using the same balance as on determination of working sample and the percentage weights were calculated using the formula presented below. Also, for morphological quantitative analysis, 1000 seeds were randomly counted from each sample and weighed. The weights ranged from 0.24-0.38 g, 1.03-1.18 g and 1.33-1.74 g, for Amaranthus hybridus, Cleome gynandra and Bidens pilosa, respectively, depending on source.

$$
\text { Percentage pure seed }=\frac{\text { Weight of pure seed after purity analysis }}{\text { Weight of working sample }} \times 100
$$


The germination tests were carried out on a working sample of 200 seeds randomly picked from a working sample (ISTA, 2016) and only intact seeds (i.e. showing no signs of damage or fungal attack, not shrivelled) were used. Hundred seeds were evenly spread on moistened filter papers- 2 replicates, laid out in a Randomised Complete Block Design on the Jacobsen tank. Plastic funnel caps were placed on each filter paper, to maintain the humidity at high levels around each seed. For Amaranthus hybridus and Cleome gynandra seeds, filter papers were moistened using potassium nitrate $\left(\mathrm{KNO}_{3}\right), 100$ seeds were evenly spread, then placed in petri dishes and pre-chilled at $5-10^{\circ} \mathrm{C}$ for seven days (ISTA, 2016). For Bidens pilosa seeds, filter papers were moistened using water and placed directly on the Jacobsen tank without pre-chilling. Germination tests were done according to ISTA standards (temperature requirements, light intensity, alternating temperatures, pre-chilling). The experiments were conducted under light (two fluorescence lamps, 40W) at 8 hours light and 16 hours darkness, alternating temperatures of $20<=30^{\circ} \mathrm{C}$, using the same Jacobsen tank throughout. Temperatures for the Jacobsen tank was monitored with readings taken at $8 \mathrm{am}, 12 \mathrm{pm}$ and 4 pm daily except for weekends and public holidays. Thereafter, seedling evaluation was done on different days depending on the prescribed requirements of ISTA and ISTA (2009) Seedling Evaluation Handbook. Germinated seeds - seedlings were counted by destructive method, with all the seedlings and diseased seeds removed on each day of counting. This included assessing each individual germinated seedling, recording on the card and classifying all normal seedlings, abnormal seedlings, fresh non-germinated, and dead seeds. Annex 3 shows the seedling evaluation intervals. Further, to substantiate whether the combination of pre-chilling and $\mathrm{KNO}_{3}$ damaged the seed, laboratory tests were carried for Amaranthus hybridus and Cleome gynandra seed in February 2017, these included testing the Amaranthus hybridus seed without pre-chilling and the use of $\mathrm{KNO}_{3}$ and pre-soaking the Cleome gynandra seed for 24 hours and 48 hours to mimic the farmer's environment and dried at ambient temperature, then evenly spread the seed on filter papers before placing them on Jacobsen tank for germination. Thereafter, similar seedling evaluation procedure described earlier were followed.

Field germination tests were conducted in Seke rural (18 $18^{\circ} 4$ 'S; 31 $312^{\circ} 9 \mathrm{E}^{\prime}$ and $1790 \mathrm{~m}$ above sea level) in December, 2016. The experiment was laid out in a Randomised Complete Block Design. The field was prepared and each treatment was clearly labelled, 100 seeds 
replicated twice were sown in a bed of 1 metre by 0.50 metres length. Guard rows were made. The seeds were placed in rows and then thinly covered with soil. The experiment was conducted under rain-fed conditions, no additional water through irrigation was applied. Seedling evaluation for Bidens pilosa was done on day 7 and 14. Amaranthus hybridus and Cleome gynandra were evaluated on days 7, 14 and 21 . Weeds were removed by hand.

Germination results were calculated using a formula derived from ISTA (2016) with modification and the ratio is then multiplied by 100 :

$$
\text { Percentage normal seedling }=\frac{\text { Normal seeds germinated }}{\text { Total number of seeds per replicate }} \times 100
$$

\section{Morphological characters evaluation of three species}

The experiment was conducted in the greenhouse at University of Zimbabwe from 17 February to 7 March, 2017. A randomized complete block design with three blocks was used. The pots were labelled and randomly arranged and filled to approximately $6 \mathrm{~cm}$ under the rim. The soil was sterilised at $70^{\circ} \mathrm{C}$ for 24 hours and allowed to cool. A mixture of soil and pine bark at ratio 1:3 was made to reduce soil compaction and placed in pots. Proper levelling of the growing media was done before approximately 1000 seeds were evenly distributed per pot. The seeds were then covered with $1.5 \mathrm{~cm}$ thick layer of the growing media. Water as needed was added to the pots. By 20 February, seeds from most pots with Amaranthus hybridus and Bidens pilosa had emerged, but not those with Cleome gynandra seeds. On 7 March, seedling emergence was high for all pots expect for Cleome gynandra pots with seed from Farmers 2 and 3 which showed very low germination. The presence of diseases on seedlings was also recorded in the greenhouse (e.g. damping off). The seedlings were thinned randomly from the pots which had high emergence. Later observations and recordings were conducted on the plants without uprooting them.

The morphological evaluation was done on 7 March at seedling development stage. UPOVInternational Union for the Protection of New Varieties of Plants (2002) standard guides were used for categorising the essential plant parts that can be used for morphological evaluation. The essential plant parts subjected to evaluation differed at different plant developmental 
stages. For instance at seedling stage, hypocotyl, colour, and pubescence can be used for morphological evaluation; when plants are full grown, the following characteristics can be used: growth habit, crown shape, root characteristics (shape, surface, flesh colour, system, leaf characteristics (e.g. blade, petiole, stipule), inflorescence, flower (e.g. calyx, sepal, corolla, petal, stamen, pistil), fruit and seed or grain. The chronological order (order of development) included seedling stage, vegetative phase, reproductive phase, pre-harvest and postharvest. UPOV (2002) guideline shows that morphological qualitative and quantitative characters of certain crop can be measured at seedling developmental stage.

The morphological characters for each species were validated using herbarium specimens in the Zimbabwe National Herbarium and Botanic Garden Institute, descriptors of PROTA (Grubben \& Denton, 2004) and the International Plant Genetic Resources Institute and descriptors listed by Wasonga et al. (2015). Only data for the seedling stage was assessed using three replicates of each species. Since the plant species were not commercial varieties like the improved maize varieties, these plant species tended to be heterogeneous (e.g. germination and population characteristics tend to vary) we recorded the most frequently occurring characteristics observed in the population. For example in the presence of anthocyanin colouration on leaf base dominated in the population, then we would evaluate that species on that basis. Similar criteria are used by ISTA, when a client submitted a mixed seed sample without declaring the name. The observations on the most frequently occurring characteristics covered seven qualitative characters - including the leaf shape, leaf size and leaf colour, stem colour and near ground stem colour, the presence of anthocyanin colouration on leaf base, presence of diseases, and one quantitative character: 1000-seed weight measured before the seed was planted. Some of these qualitative morphological characters have also been used by Blas et al. (2008) and listed by Wasonga et al. (2015) in morphological characterisation of wild Arracacia species and Cleome gynandra, respectively.

\section{Data Analysis}

The analysis of variance (ANOVA) in GenStat $18^{\text {th }}$ edition was performed on percentage germination, abnormal seedling, fresh non-germinated seed, dead seed and 1000-seed weights and also to test the significance of the differences among the seed sources per species. All the results were transformed using LOG10 plus 1, except for 1000 -seed weights 
and purity of the seed. The transformation was done because some of the data sets had zero values. All the statistical analyses for germination results were carried out on the transformed data. Means of the treatments within each species, among seed sources by species were separated by Duncan's multiple range least significance difference at $5 \%$ (LSD.05). The independent variables included: age, source, versus percentage germination, abnormal seedling, fresh non-germinated and dead, as dependent variables. For purity analysis results were visually compared among the seed sources per species. For morphological analysis, only the quantitative morphological character (1000-seed weight) data was subjected to ANOVA and means separation. 


\subsection{Results}

The Seeds Rules and Regulations (1971) of Zimbabwe does not have guidelines for seed certification for these plant species; they are only regarded as weeds (Seeds Act (1971)). Hence, there were no prescribed standards for these plant species to compare with. Normal standards are presented on Seeds [certification Scheme] Notice (2000), e.g. maize percentage germination and purity is $90 \%$ and $99 \%$, respectively. With the case of TLV seeds not covered, but from the farmer's perspective, seed with high germination is preferable. Seed samples sourced from farmers and research organization were tested for germination in both laboratory and field. The percentage germination and purity were compared among the seed sources and the tests and results are presented in figure and table form. Generally, Cleome gynandra germination was poor; the species had difficulties in germination and was characterised by high occurrence of fresh non-germinated seed at the end of each germination test.

Table 9 shows the percentage pure seed for traditional leafy vegetable species by farmer. Highest percentage pure seed was observed on seed sourced from Farmer 4 for Amaranthus hybridus. The lowest percentage pure seed for Cleome gynandra was observed for Farmer 1, followed by Farmer 4 and highest for Farmer 3. For Bidens pilosa the highest percentage pure seed was detected for Farmer 3 and least for Farmer 1. However, the differences among farmers are very small for all three species.

Table 9 The percentage pure seed for traditional leafy vegetables from five farmers (in parentheses the transformed values)

$\begin{array}{llll}\text { Source } & \text { Amaranthus hybridus } & \text { Cleome gynandra } & \text { Bidens pilosa } \\ \text { Farmer 1 } & 95.3(1.98) & 94.2(1.97) & 97.7(1.99) \\ \text { Farmer 2 } & 89.3(1.95) & 99.8(2.00) & 99.6(2.00) \\ \text { Farmer 3 } & 85.7(1.93) & 99.9(2.00) & 99.9(2.00) \\ \text { Farmer 4 } & 99.6(2.00) & 96.4(1.98) & 99.6(2.00) \\ \text { Farmer 5 } & 90.6(1.96) & 99.8(2.00) & 98.0(1.99)\end{array}$


Table 10 depicts the percentage mean dead seed for traditional leafy vegetable species by farmer. There were no significance differences on dead seed percentage across all the seed sources by species.

Table 10 The mean percentage dead seeds for traditional leafy vegetable from five farmers (in parentheses the transformed values and the mean separation)

$\begin{array}{llll}\text { Source } & \text { Amaranthus hybridus } & \text { Cleome gynandra } & \text { Bidens pilosa } \\ \text { Farmer 1 } & 20(1.06 a) & 17(1.03 a) & 13(1.08 a) \\ \text { Farmer 2 } & 22(1.14 a) & 19(1.04 a) & 13(1.08 a) \\ \text { Farmer 3 } & 15(0.96 a) & 20(1.04 a) & 10(0.94 a) \\ \text { Farmer 4 } & 14(0.97 a) & 16(0.97 a) & 9(0.94 a) \\ \text { Farmer 5 } & 13(0.89 a) & 12(0.86 a) & 8(0.92 a) \\ \text { SSD }_{5 \%} & 0.35 & \mathbf{0 . 3 0} & \mathbf{0 . 1 8}\end{array}$

The mean separation indicates per species the differences among the five farmers

Table 11 shows the percentage mean fresh non-germinated seed for traditional leafy vegetable species by farmer. The percentage fresh non-germinated seed differed among the farmers but not for Cleome gynandra, which did not show any significant differences across all the seed sources. For Amaranthus hybridus, Farmers 2, 3, 4, and 5 showed highest percentage mean fresh non-germinated seed and did not significantly differ among themselves. The lowest percentage fresh non-germinated seed was observed for Farmer 1, but did not significantly differ from Farmer 5 . The results for the percentage fresh nongerminated seeds for Bidens pilosa also showed that Farmers 2, 3, 4, and 5 were not significantly different and least. However, Farmers 1 and 2 did not differ significantly and depicted highest percentages fresh non-germinated seed. 
Table 11 The mean percentage fresh non-germinated seeds for traditional leafy vegetables from five farmers (in parentheses the transformed values and the mean separation)

$\begin{array}{llll}\text { Source } & \text { Amaranthus hybridus } & \text { Cleome gynandra } & \text { Bidens pilosa } \\ \text { Farmer } 1 & 4(0.63 \mathrm{a}) & 74(1.68 \mathrm{a}) & 11(0.84 \mathrm{~b}) \\ \text { Farmer } 2 & 26(1.16 \mathrm{~b}) & 77(1.71 \mathrm{a}) & 6(0.60 \mathrm{ab}) \\ \text { Farmer } 3 & 50(1.31 \mathrm{~b}) & 77(1.70 \mathrm{a}) & 3(0.43 \mathrm{a}) \\ \text { Farmer } 4 & 24(1.12 \mathrm{~b}) & 70(1.66 \mathrm{a}) & 2(0.44 \mathrm{a}) \\ \text { Farmer } 5 & 23(1.09 \mathrm{ab}) & 68(1.65 \mathrm{a}) & 2(0.36 \mathrm{a}) \\ \text { LSD }_{5 \%} & \mathbf{0 . 4 6} & \mathbf{0 . 4 7} & \mathbf{0 . 3 4}\end{array}$

The mean separation indicates per species the differences among the five farmers

Percentage germination for the traditional leafy vegetable species

The germination for the traditional leafy vegetable species by farmers were evaluated and results are presented in Figure 1-3. For all species, the percentage germination refers to the count of normal seedlings classified according to ISTA (2016), Seedling Evaluation Hand Book. Seedlings are regarded as normal if they are defects free, and have the ability to grow into a healthy plant under conducive environments. The percentage germination for all the traditional leafy vegetable species increased at each time interval as storage duration increased from August to November. In the case of each species, the percentage germination varied among farmers. The details for each species are presented later. 
For Amaranthus hybridus Figure 2, seed sourced from Farmer 3 consistently showed the lowest germination percentage across all time intervals, except for February. In comparison among farmers, Farmers 2, 3, 4, and 5, had considerably very low percentage germination during the first four months of storage. The highest percentage germination was observed for Farmer 1. Interestingly, for Farmer 1, the percentage germination drastically went down, whereas Farmers 3, 4 and 5 a sharp increase was realised on the seventh month during storage. The standard deviation for each farmer are; Farmer 1 is 9 , Farmer is 12 , Farmer 3 is 35, Farmer 4 is 16 and Farmer 5 is 18.

Figure 2 The percentage germination for Amaranthus hybridus from five farmers

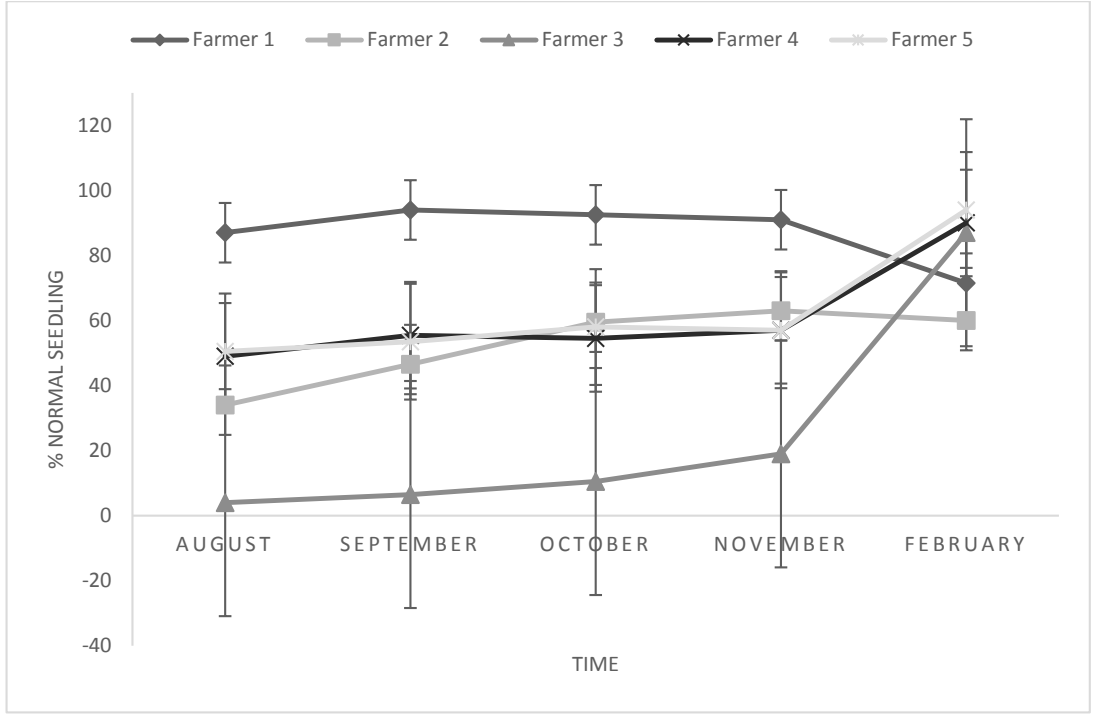

Source: Author. 
Figure 3 depicts very low but variable percentages of germination for Cleome gynandra. The highest percentage germination was consistently observed for Farmer 5, intermediate for Farmer 4 and least for Farmers 2 and 3. There was a sharp increase in percentage germination for Farmers 4 and 5 between November and February. The percentage germination for Farmer 1 showed consistent trends, maintaining a slight increase by month, throughout the tests. The standard deviations are presented by farmer with the following values; Farmer is 1, Farmers 2 and 3, is 0, Farmer 4 is 7 and Farmer 5 is 13.

Figure 3 The percentage germination for Cleome gynandra from five farmers

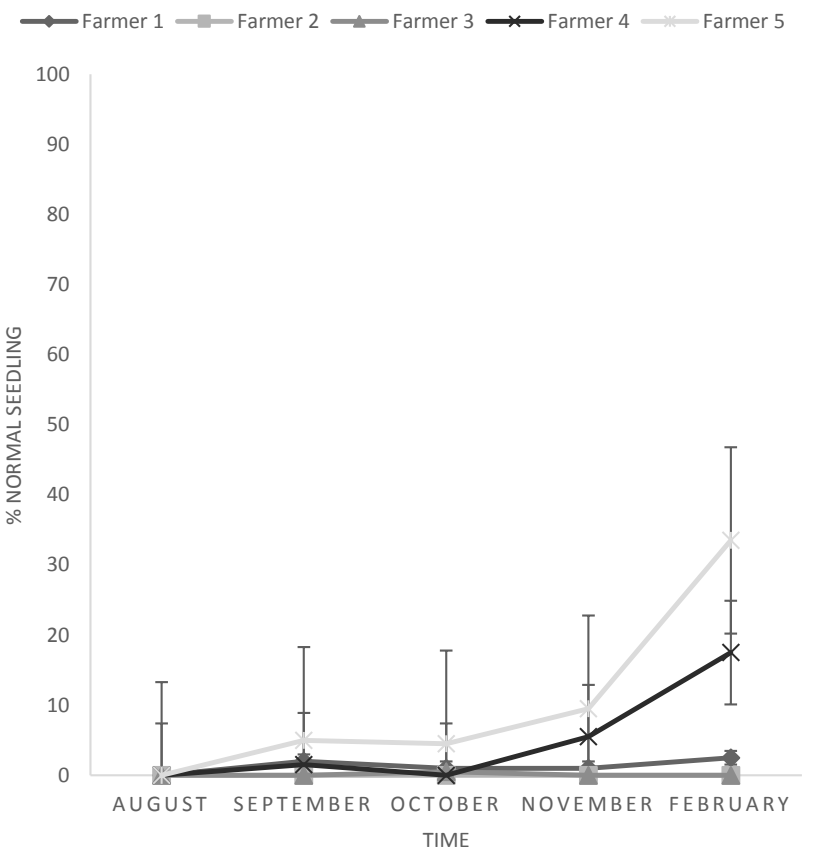

Source: Author. 
For Bidens pilosa, Figure 4, percentages germination were higher across all the seed sources compared to other species; the age effect did not show very consistent and distinctive trends, especially not for Farmers 3, 4 and 5. However, distinctive trends were observed for Farmers 1 and 2 . The standard deviation are presented in the figure by farmer and include the following values; Farmer 1 is 13, Farmer 2 is 6 , Farmer 3 has 4, Farmers 4 and 5 is 1.

Figure 4 The percentage germination for Bidens pilosa from five farmers

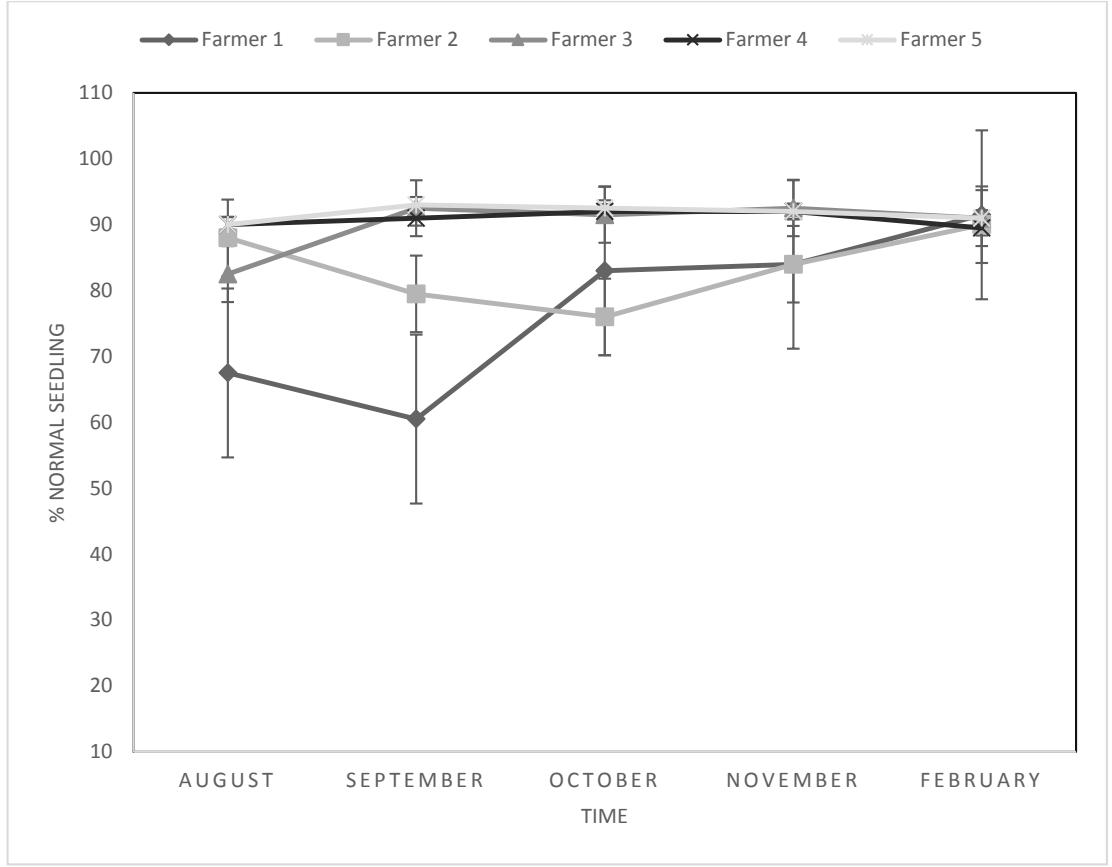

Source: Author. 
The two-way interactions between time and method on percentage germination (full results see Annex 5 to Annex 7)

The results of a two-way ANOVA for Amaranthus hybridus (Annex 5) did not show a clear trend on the percentage germination. However, pre-chilling $+\mathrm{KNO}_{3}$ and water in February gave the highest percentage germination; these values were, however, not statistically significant from those of October and November. The results obtained from the field were significantly lower than the values from laboratory tests, but were not statistically significantly different from the results obtained in August, September, October and November where prechilling and $\mathrm{KNO}_{3}$ were used. For Cleome gynandra (Annex 6), pre-soaking the seed for 24 and 48 hours before planting led to highest percentage germination in February. The ranking of percentage germination using pre-chilling and $\mathrm{KNO}_{3}$ was consistent among the planting times, but February gave highest percentage germination, least in August. The field results for Cleome gynandra gave statistically significantly higher percentages germination over prechilling and $\mathrm{KNO}_{3}$. The difference between methods and time on percentage germination for Bidens pilosa (Annex 7) was statistically significant. The field tests gave significantly lower results comparable to the results obtained in laboratory tests for August and September.

Qualitative and quantitative morphological characters evaluation of three species of traditional leafy vegetables

The qualitative morphological characters of each traditional leafy vegetable species were examined among the farmers presented (full results see Annex 4). We observed high morphological character variations in Amaranthus hybridus seed. Only Amaranthus hybridus seed for Farmer 2 had narrow, lanceolate leaves while the remaining four seed sources had broad, ovate leaves. Farmer 3 had a majority of seedlings with red purple stem. The majority of farmers had seedlings without anthocyanin colouration on leaf base except for Farmer 4 who had seed producing plants that had anthocyanin colouration on the leaf base. Damping off was observed on Amaranthus hybridus and Cleome gynandra on seed from Farmer 4 only. No morphological character differences were observed for seed from the five farmers on Cleome gynandra and Bidens pilosa. 
The weights of 1000 randomly selected seeds per plant species by farmer are presented in Table 12 and were used to determine the quantitative morphological characters by performing Analysis of Variance and separation of means using Duncan's multiple range method in GenStat $\left(L S D_{5 \%}\right)$.

There were significant differences among the mean weights of 1000 seeds of Amaranthus hybridus, Cleome gynandra and Bidens pilosa for farmers (Figure 12). For both Amaranthus hybridus and Cleome gynandra species, seeds from Farmer 1 had the lightest ones $(0.24 \mathrm{~g}$ and $1.03 \mathrm{~g}$, respectively). For Bidens pilosa Farmer 3 had the lightest weight of $1.33 \mathrm{~g}$.

Table 12 Quantitative morphological characters of three species of traditional leafy vegetables

Source

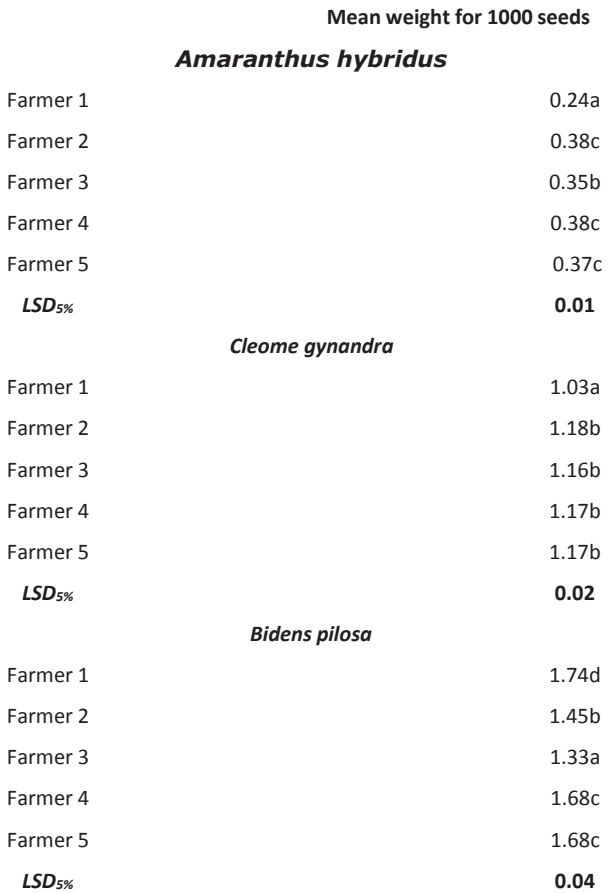

For each factor, different letters indicate significant differences according to Duncan's multiple range least significant difference test $\left(L S D_{5 \%}\right)$ for each plant species.

Source: Author 


\subsection{Discussion}

Seed is the initial link in the food production chain (Shiva, 2016); good seed perpetuates to more yield and yields better-quality products (Louwaars \& Boef, 2012). Seed also symbolizes food security attainment (Shiva, 2016). Good seed comprises of many different attributes that add to the performance of the seed ranging from being of proper age, being able to give a vigorous and uniform crop stand and producing vigorous plants that give an optimal yield, being genetically pure to avoid an uneven crop stand and difficulties with harvest if crop plants mature unevenly (Madamba, 2002; Dube \& Mujaju, 2013), enabling farmers to meet the requirements of the market (Karanja et al., 2012), e.g. regarding taste, market preference and other quality aspects. In this study, the term seed quality refers to the physiological characteristics, purity and the uniformity of the plants.

TLV seeds collected from five different farmers were compared for percentage germination and purity, and qualitative and quantitative morphological characteristics. Exposure to alternating temperatures of $20<=>30^{\circ} \mathrm{C}$, exposure to light, treatment with $\mathrm{KNO}_{3}$ and prechilling are treatments that are predominantly used to improve the germination of many seed crops. Such crops include Amaranthus hybridus and Cleome gynandra (ISTA, 2016) and we therefore used those treatments in our research approach. Pre-soaking treatment can also be used to start the process of germination (Ashraf \& Abu Shakra, 1978) and was therefore also used to improve the germination for Cleome gynandra seed. For Bidens pilosa we used water to moisten filter papers and alternating temperatures of $20<=>30^{\circ} \mathrm{C}$ to enhance germination.

The least percentage germination was observed for Cleome gynandra throughout the tests, intermediate values were observed for Amaranthus hybridus and highest values for Bidens pilosa seed. Throughout the tests, Cleome gynandra seed showed high levels of dormancy, a feature which is potentially disastrous to farmers. However, the results for Bidens pilosa described in this paper were within the range reported by Dube \& Mujaju (2013). All results showed that the germination percentage increased with age and varied with the test method used when no dormancy was present. 
On average, using $\mathrm{KNO}_{3}$ solution to moisten filter papers and pre-chilling Cleome gynandra seed for seven days at $5-10^{\circ} \mathrm{C}$ gave lower results than the field test, pre-soaking seed for 24 and 48 hours. According to Kim et al. (2008), light induces the reduction of abscisic acid (ABA) which in turn promotes germination in many plant species. For Cleome gynandra seed, it was observed that such effect did not promote germination, rather induced dormancy. Similar results were reported by Ochuodho \& Modi (2007), who observed that the germination of Cleome gynandra seed tested at $20^{\circ} \mathrm{C}$ was inhibited by light, and gave $0 \%$ germination. According to Copeland \& McDonald (1995), seeds that are sensitive to light are also sensitive to $\mathrm{KNO}_{3}$. The seed from all farmers showed very low significant percentage germination. Taken together, our results show that Cleome gynandra seed presents both $\mathrm{KNO}_{3}$ and light sensitivity throughout the tests. Furthermore, for Cleome gynandra, it was observed that prechilling seed for seven days at $5-10^{\circ} \mathrm{C}$ would not improve percentage germination. According to ISTA (2016), pre-chilling is a way of breaking the seed dormancy, thereby improve percentage germination in many crop species including Cleome gynandra. There are reports of pre-chilling sensitivity in seed of wild plant species, such as Withania somnifera (Kambizi et al., 2006) and texasweed seed (Koger et al., 2004). Koger et al. (2004) found that pre-chilling the texasweed seed for 1 day resulted in a decrease in germination to $17 \%$ compared to higher percentage germination obtained without any pre-chilling. Pre-chilling Cleome gynandra seed for seven days at $5-10^{\circ} \mathrm{C}$ and hydration for four hours significantly reduced the germination (Ochuodho \& Modi, 2005). In comparison between the tests, seed pre-soaked for 24 or 48 hours in water, though relatively low, had significantly higher percentage germination than observed in the $\mathrm{KNO}_{3}$ treatment. Although our findings for the field tests were very low compared to the results reported by Abukutsa-Onyango (2007), they were significantly higher than in the laboratory tests where a combination of $\mathrm{KNO}_{3}$, pre-chilling, light and alternating temperatures of $20<=30^{\circ} \mathrm{C}$ were used. The field results also significantly differed among the seed sources. Abukutsa-Onyango (2007) reported $90 \%$ and $30 \%$ germination of Cleome gynandra seed tested in the field and laboratory, respectively. Cleome gynandra seeds from Farmers 5, 4, and 1, in that order, for instance showed significantly higher percentages of germination for all tests, no significant variation on percentage fresh non-germinated seed, showing that this phenomena cannot be merely attributed to species but is also influenced by environmental effects. Our results can be partially explained by findings by Ochuodho \& Modi (2005) and de Barros et al. (2017): they confirmed that seed 
dormancy relates to different habitats from where the seeds were collected. Also there was germination variation among seeds sown in the greenhouse for morphological analysis (results not shown), confirming our results from the laboratory. Seed sourced from Farmers 2 and 3 had very low germination, hardly 20 plants per pot among all three replicates, although approximately 1000 seeds were used per each pot.

For Amaranthus hybridus seed, moistening filter papers with water gave comparable results with pre-chilling for seven days at $5-10^{\circ} \mathrm{C}$ with filter papers moistened by a solution of $\mathrm{KNO}_{3}$. Germination percentages were significantly higher than in the field test. The average percentage germination varied among farmers and test method used. The higher variations in Amaranthus hybridus seed can be the result of environmental effects and genetic variation as already shown for the morphological evaluations we conducted. The results for the morphological evaluations showed a high diversity among the Amaranthus hybridus species. The magnitude of morphological variations among the seed sources suggests that the population must be heterogeneous, since we recorded the most frequently occurring characteristics from the observation. This finding can partially explain the observed variation in percentage germination as well.

For Bidens pilosa, laboratory tests gave significantly higher results than the field test. Generally, Bidens pilosa seed germination is not problematic. The low percentage germination observed in the field test could have been caused by excessive rains received just after planting (over $100 \mathrm{~mm}$ down pour on that day). The average percentages germination were statistically significant among farmers. Bidens pilosa seed germination depends on seed origin (de Barros et al., 2017). The average percentage germination and purity of the seed across all seed sources were higher than earlier reported by Dube \& Mujaju (2013) for Bidens pilosa. These authors reported the minimum germination and purity standards to be $88 \%$ and $99 \%$ respectively.

There were significant differences in the weight of 1000 seeds between farmers and between species. For Cleome gynandra the weight for 1000 seeds ranged from $1.03 \mathrm{~g}$ (Farmer 1) to $1.18 \mathrm{~g}$ (Farmer 2 ) and weights statistically differed by farmer $(p=0.05 \%)$. Seed weight for 1000 seeds of Amaranthus hybridus was statistically significant $(p=0.05 \%)$ among all farmers, 
lowest weight observed was $0.24 \mathrm{~g}$ (Farmer 1 ) and heaviest was $0.38 \mathrm{~g}$ (Farmer 2). Also, 1000seed weight of Bidens pilosa was statistically significantly different $(p=0.05 \%)$ among all farmers, ranging from $1.33 \mathrm{~g}$ (Farmer 3) to $1.74 \mathrm{~g}$ (Farmer 1). The observed 1000-seed weights for Cleome gynandra are within the range to those obtained from seed sourced in communities in western Kenya (Abukutsa-Onyango, 2007). Bidens pilosa seed produced under optimum conditions had heavier weights (de Barros et al., 2017). These 1000-seed weight differences can be attributed to the different agro ecological conditions and other environmental conditions prevailing during seed formation, but could also due to different harvest dates, to genetic variability between the populations as already confirmed by the qualitative morphological analysis and also varying seed moisture content. In our study, we did not test for moisture content, though. For instance, Farmer 5, Amaranthus hybridus and Bidens pilosa were planted early December, the flowering stage could have coincided with low rainfall experienced starting from January onwards.

The classification of species according to stem colour has been reported to be very useful to the farmers and consumers. The purple pigments on the stem is very useful and has been reported to play a role in deterring pests (Schippers, 2000). The Cleome gynandra with purple stem is less attractive to pests, drought tolerant and more medicinal than the green stemmed varieties. The author further reported that the purple pigments provide quite a range of health benefits to people, including much needed antioxidants. Other people preferred a green stem because of its tenderness and less bitter taste compared to the purple stem which is bitterer and takes more time to cook. Another important attribute concerning the green stemmed type is that it regenerates better than the purple type, the latter has a problem of drying up or decaying when the major shoots have been harvested only once or twice (Schippers, 2000). 


\subsection{Conclusions and recommendations}

Cleome gynandra seeds germinated better after pre-soaking for 24 and 48 hours and in field conditions. Hence, pre-soaking Cleome gynandra seeds can be important to kick start the germination process and is one of the good standard procedures to test the quality of this species. The method is relatively inexpensive and even used by resource-poor smallholder farmers. For Bidens pilosa seeds, applying more water than required whether in the field or in the germination trays in the laboratory poses risks of poor percentage germination. However, water can be used to moisten the filter papers instead of $\mathrm{KNO}_{3}$ treatment. Amaranthus hybridus seeds had moderately high percentage germination, but better germination can be obtained after prolonged storage. Likewise, water can be used to the filter papers instead of $\mathrm{KNO}_{3}$ treatment and pre-chilling is required to improve germination. This study hypothesizes that percentage germination varies among seed sources. For Cleome gynandra and Bidens pilosa seeds, seeds sourced from Farmer 5 gave highest percentage germination, showing that the seeds were of good quality. Amaranthus hybridus seeds from Farmer 1 had highest percentage germination. The heterogeneous population observed for Amaranthus hybridus seed indicates the rich genetic pool that can be used for breeding purposes and improvement of the species through selection at farmers' level.

\section{Acknowledgements}

This research was possible through the support of the Dutch Government through the Netherlands Fellowship Programme. Thank you to Head of Seed Services, Seed Analysts and Research Officers, you made my work more achievable and enjoyable. 


\section{References}

Abukutsa-Onyango, M. (2007). Seed production and support systems for African leafy vegetables in three communities in western Kenya. African Journal of Food, Agriculture, Nutrition and Development. 7: 1-16.

Adebooye, O. C., Ajayi, S. A., Baidu-Forson, J. J. \& Opabode, J. T. (2005). Seed constraint to cultivation and productivity of African Indigenous leaf vegetables. African Journal of Biotechnology. 4 (13): 1480-1484.

Afari-Sefa, V., Chagomoka, T., Karanja, D. K., Njeru, E., Samali, S., Katunzi, A., Mtwaenzi, H. \& Kimenye, L. (2013). Private contracting versus community seed production systems: Experiences from farmer-led seed enterprise development of indigenous vegetables in Tanzania. Acta Horticulturae. 1007 (1): 671-680.

Ambrose-Oji, B. (2009). Urban Food Systems and Traditional Leafy Vegetables: Defining the Spaces and Places for Traditional Leafy Vegetables in Urban and Peri-Urban Agriculture. In: Traditional Leafy Vegetables in urban agriculture. (Shackleton C.M., Pasquini M.W., Drescher A.W., eds.) London, Earthscan, $1-33$.

Ashraf, C. M. \& Abu-Shakra, S. (1978). Wheat seed germination under low temperature and moisture stress. Agronomy Journal (Abstract). 70 (1): 135-139.

Ayana, A., Afari-Sefa, V., Emana, B., Dinssa, F. F., Balemi, T. \& Temesgen, M. (2014) Analysis of Vegetable Seed Systems and Implications for Vegetable Development in the Humid Tropics of Ethiopia. International Journal of Agriculture and Forestry. 4 (4): 325-337.

Biemond, P. C., Stomph, T. J., Kamara, A., Abdoulaye, T., Herane, S. \& Struik, P. C. (2012). Are investments in an informal seed system for cowpeas a worthwhile endeavour? International Journal of Plant Production. 6 (3): 367- 386.

Blas, R., Ghislain, M., Herrera, M. R. \& Baudon, J-P. (2008). Genetic diversity analysis of wild Arracacia species according to morphological and molecular markers. Genetic Resources and Crop Evolution. 55: 625-642.

Cernansky, R. (2015). Super Vegetables Long Overlooked in Parts of Africa, Indigenous Greens Are Now Capturing Attention for their Nutritional and Environmental Benefits. Nature. 522: 146-148. 
Chweya, J. A. \& Mnzava, N. A. (1997). Promoting the conservation and use of underutilized and neglected crops. 11 . Cat's whisker@leome gynandra L. International Plant Genetic Resources Institute, Rome, Italy. 1-54.

Copeland, L. O. \& McDonald, M. B. (1995). Principles of seed science and technology. $3^{\text {rd }}$ edition. Chapman \& Hall. 127-152.

Copeland, L. O. \& McDonald, M. B. (2001). Seed Pathology and Pathological Testing. Chapter 16. Book Principles of Seed Science and Technology Publisher Springer US: 354-379.

Cromwell, E., Esbern, F. H. \& Turner, M. (1992). The seed sector in developing countries: a Frame-work for performance analysis. Overseas Development Institute (ODI) Working Paper 65. London, UK. Available at: http://www.odi.org/sites/odi.org.uk/files/odi assets/publications-opinion-files/6969.pdf. Accessed 17 October, 2017. 1-118.

de Barros, R.T., Martins, C. C., da Silva, G. Z. \& Martins, D. (2017). Origin and temperature on the germination of beggartick seeds. Revista Brasileira de Engenharia Agrícola e Ambiental. 21 (7): 448-453.

DFID Research4Development Project Record (2003). Improving the livelihoods of peri-urban vegetable growers through markets promotion of fresh and processed indigenous vegetables R4D Project URL.

Dube, P. \& Mujaju, C. (2013). Determination of standards for purity and germination for African Indigenous Vegetable (AIV), Blackjack (Bidens pilosa). Advanced Journal of Agricultural Research. 1 (003): 32-38.

Grubben, G. J. H. \& Denton, O. A. (Editors), (2004). Plant Resources of Tropical Africa 2. Vegetables. PROTA Foundation, Wageningen, Netherlands / Backhuys Publishers, Leiden, Netherlands / CTA, Wageningen, Netherlands. 1-668.

ISTA (2009). ISTA Handbook on Seedling Evaluation Handbook, Third Edition with Amendments Bassersdorf, Switzerland.

ISTA (2016). International Rules for Seed Testing, Edition 2016. International Seed Testing Association (ISTA), Zurich, Switzerland.

Jacobsen, S. E. \& Mujica, A. (2014). The genetic resources of Andean grain amaranths (Amaranthus caudatus L., A. cruentus L. and A. hipochondriacus L.) in America. Bioversity International - FAO, Newsletter. 133: 41-44. 
Kambizi, L., Adebola, P.O. \& Afolayan, A. J. (2006). Effects of temperature, pre-chilling and light on seed germination of Withania somnifera; a high value medicinal plant. South African Journal of Botany. 72: 11-14.

Karanja, D., Okoko, N., Kiptarus, E., Okongo, P., Samali, S., Katunzi, A., Mtwaenzi, H., Mwakitwang, F., AfariSefa, V., Musebe, R., Kimani, M. \& Kimenye, L. (2012). Promoting farmer-led seed enterprises of African indigenous vegetables to boost household incomes and nutrition in Kenya and Tanzania. Conference on Agricultural Biodiversity in Africa: 1-13.

Kim, D. H., Yamaguchi, S., Soohwan, L., Oh, E., Park, J., Hanada, A., Kamiya, Y. \& Choi, G. (2008). Somnus, a $\mathrm{CCCH}$-Type zinc finger protein in Arabidopsis, negatively regulates lightdependent seed germination downstream of PIL5. The Plant Cells. 20: 1260-1277.

Koger, C. H., Reddy, K. N. \& Poston, D. H. (2004). Factors affecting seed germination, seed emergence and survival of texasweed (Caperonia palustris). Weed Science. 52: 989995.

Linhart, Y. B. \& Grant, M. C. (1996). Evolutionary significance of local genetic differentiation in plants. Annual Review of Ecology and Systematics Journal. 27: 237-277.

Louwaars, N. P. \& De Boef, W. S. (2012). Integrated Seed Sector Development in Africa: A Conceptual Framework for Creating Coherence Between Practices, Programs, and Policies. Journal of Crop Improvement. 26: 39-59.

Madamba, R. (2002). (Nyakanda, P. and Hikwa, D. eds.) Producing cowpea seed. Handbook. The Crop Breeding Institute. Department of Research and Specialist Services, Harare, Zimbabwe.

Masuka, A., Goss, M. \& Mazarura, U. (2012). Morphological characterisation of four selected spider plant (C. gynandra L.) Morphs from Zimbabwe and Kenya. Asian Economic and Social Society. Asian Journal of Agriculture and Rural Development. 2 (4): 646-657.

Ngwerume, F. C. \& Mvere, B. (2003). Report on the findings of a socio-economic survey on the marketing, consumption and production of traditional vegetables in the urban and peri urban areas of Harare, Zimbabwe. DR \& SS-HRC/NRI project a0892.

Ochuodho, J. O. (2005). Physiological basis of seed germination in Cleome gynandra L. PhD Thesis. University of KwaZulu-Natal, Pietermaritzburg, South Africa.

Ochuodho, J. O. \& Modi, A. T. (2005). Temperature and light requirements for the germination of Cleome gynandra seeds. South African Journal of Plant and soil. 22 (1): 49-54. 
Ochuodho, J. O. \& Modi, A. T. (2007). Light-induced transient dormancy in Cleome gynandra L. seeds. African Journal of Agricultural Research. 2 (11): 587-591.

Ojiewo, O. C., Mwai, G. N., Abukutsa-Onyango, M. O., Agong, S. G. \& Nono-Womdim, R. (2012) Exploiting the genetic diversity of vegetable African Nightshades. Bioremediation, Biodiversity and Bioavailability, Global Science Books. 7 (1): 6-13.

Oniang'o, R. K., Shiundu, K., Maunda, P. \& Johns, T. ( $\left.\begin{array}{llll}2 & 0 & 0 & 6\end{array}\right)$. Diversity, nutrition and food security: The case study of African Leaf Vegetables, in: Hunger and Poverty: The role of biodiversity, Bala Ravi, S. Hoeschle-Zeledon, I., Swaminathan, M. S. \& Frison, E. (eds) Rome, Italy: IPGRI: 86-100.

Schippers, R. R. (2000). Traditional Leafy Vegetables. An overview of the cultivated species. Chatham, UK: Natural Resources Institute/ACP-EU Technical Centre of Agricultural and Rural Cooperation Book: 1-210.

Seeds Act [Chapter 19:13], (1971). Print flow, Government printers, Harare, Zimbabwe. http://www.parlzim.gov.zw/attachments/article/105/SEEDS_ACT_19_13.pdf

Seeds Rules and Regulations, (1971). Print flow, Government printers, Harare, Zimbabwe.

Seeds [Certification Scheme] Notice, (2000). Print flow, Government printers, Harare, Zimbabwe.

Shiva, V. (2016). Stolen harvest. The hijacking of the global food supply. University Press of Kentucky. 1-153.

UPOV (2002). International Union for the Protection of New Varieties of Plants. General Introduction to the examination of Distinctness, Uniformity and Stability and the Development of Harmonized Descriptions of New Varieties of Plants. TG/1/3 Geneva. 1-26.

Wasonga, D. O., Ambuko, J. L., Chemining'wa, G. N., Odeny, D. A. \& Crampton, B. G. (2015). Morphological Characterization and Selection of Spider Plant (Cleome Gynandra) Accessions from Kenya and South Africa. Asian Journal of Agricultural Sciences. 7 (4): 36-44.

Zharare, G. E. (2012). Differential requirements for breaking seed dormancy in biotypes of $C$. gynandra and two Amaranthus species. African Journal of Agricultural Research. 7 (36): 5049-5059.

https://www.worldweatheronline.com Historical average weather 20/04/2017 
Annex

Annex 1 Average monthly weather data (temperature, rainfall, cloud cover, humidity and bees activity

\begin{tabular}{|c|c|c|c|c|c|c|c|}
\hline \multirow[t]{2}{*}{ Weather } & Dec & Jan & Feb & March & April & May & June \\
\hline & \multicolumn{7}{|c|}{ Farmer 1} \\
\hline Temp $\left({ }^{\circ} \mathrm{C}\right)$ & 31 & 29 & 30 & 28 & 26 & 23 & 22 \\
\hline Rain (mm) & 73 & 56 & 49 & 98 & 68.5 & 11.2 & 0.31 \\
\hline$\%$ Cloud cover & 24 & 36 & 27 & 33 & 25 & 17 & 5 \\
\hline$\%$ Humidity & 57 & 69 & 72 & 81 & 68 & 61 & 56 \\
\hline Bees activity & - & - & $\bmod$ & $\bmod$ & high & Low & - \\
\hline \multirow[t]{2}{*}{ Planting } & \multicolumn{7}{|c|}{ Sole crop } \\
\hline & \multicolumn{7}{|c|}{ Farmer 2} \\
\hline Temp $\left({ }^{\circ} \mathrm{C}\right)$ & 31 & 29 & 30 & 28 & 26 & 22 & 21 \\
\hline Rain (mm) & 100 & 49 & 46 & 85 & 29.5 & 17 & 0.8 \\
\hline$\%$ Cloud cover & - & 39 & 33 & 38 & 22 & 20 & 13 \\
\hline$\%$ Humidity & - & 67 & 76 & 79 & 69 & 67 & 63 \\
\hline Bees activity & - & - & - & $\bmod$ & high & Low & - \\
\hline \multirow[t]{2}{*}{ Planting } & \multicolumn{7}{|c|}{ Intercropped with maize } \\
\hline & \multicolumn{7}{|c|}{ Farmer 3} \\
\hline $\operatorname{Temp}\left({ }^{\circ} \mathrm{C}\right)$ & 31 & 29 & 30 & 28 & 26 & 22 & 21 \\
\hline Rain (mm) & 100 & 49 & 46 & 85 & 29.5 & 17 & 0.8 \\
\hline$\%$ Cloud cover & - & 39 & 33 & 38 & 22 & 20 & 13 \\
\hline$\%$ Humidity & - & 67 & 76 & 79 & 69 & 67 & 63 \\
\hline Bees activity & - & - & $\bmod$ & $\bmod$ & high & Low & - \\
\hline \multirow[t]{2}{*}{ Planting } & \multicolumn{7}{|c|}{ Intercropped with maize } \\
\hline & \multicolumn{7}{|c|}{ Farmer 4} \\
\hline $\operatorname{Temp}\left({ }^{\circ} \mathrm{C}\right)$ & 31 & 29 & 30 & 28 & 26 & 22 & 21 \\
\hline Rain $(\mathrm{mm})$ & 110 & 57 & 63 & 29 & 15 & 10 & 1.5 \\
\hline$\%$ Cloud cover & - & 39 & 33 & 38 & 22 & 20 & 13 \\
\hline$\%$ Humidity & - & 67 & 76 & 79 & 69 & 67 & 63 \\
\hline Bees activity & - & - & $\bmod$ & $\bmod$ & high & - & - \\
\hline \multirow[t]{2}{*}{ Planting } & \multicolumn{7}{|c|}{ Sole crop } \\
\hline & \multicolumn{7}{|c|}{ Farmer 5} \\
\hline $\operatorname{Temp}\left({ }^{\circ} \mathrm{C}\right)$ & 28.9 & 27.2 & 28.4 & 26.7 & - & - & - \\
\hline Rain (mm) & 105.7 & 14.5 & 16.7 & 24.8 & - & - & - \\
\hline$\%$ Cloud cover & 24 & 33 & 30 & 36 & 29 & 21 & 17 \\
\hline$\%$ Humidity & 57 & 68 & 67 & 77 & 70 & 69 & 64 \\
\hline Bees activity & - & - & - & - & - & - & - \\
\hline Planting & \multicolumn{7}{|c|}{ Sole crop } \\
\hline
\end{tabular}

Source: Authors \& world weather online.com 
The research organization is a government institute located in Mashonaland East province and is under agro ecological region $2 \mathrm{~B}\left(18^{\circ} 11^{\prime} 6.97^{\prime \prime} \mathrm{S}: 31^{\circ} 33^{\prime} 6.95^{\prime \prime} \mathrm{E}\right)$ at an elevation of 1688 $\mathrm{m}$ above sea level. The mean annual rainfall for the area ranges from $850-900 \mathrm{~mm}$. The mean annual temperature ranges from $18-29^{\circ} \mathrm{C}$. During seed crop growth temperatures and rainfall ranged (December 2015- March 2016) 28.9-26.7 ${ }^{\circ} \mathrm{C}$ and 105.7-24.8 mm, respectively (full detail see annex 1). Shamva is in Mashonaland Central province and located under agro ecological region $3\left(17^{\circ} 18^{\prime} 56^{\prime \prime} \mathrm{S}\right.$ : $\left.31^{\circ} 34^{\prime} 14^{\prime \prime} \mathrm{E}\right)$ at an elevation of $953 \mathrm{~m}$ above sea level, receiving the annual rainfall of the range $800-850 \mathrm{~mm}$. The mean temperature for Shamva ranges between $21-31{ }^{\circ} \mathrm{C}$. During seed crop growth temperatures and rainfall ranged (December 2015- June 2016) $31-21^{\circ} \mathrm{C}$ and $110-1.5 \mathrm{~mm}$, respectively (annex 1). Murehwa is located in Mashonaland East province under agro ecological region 3 (17 38' 35.59" S: 31 ${ }^{\circ}$ $47^{\prime} 2.40 " \mathrm{E}$ ) at an elevation of $1400 \mathrm{~m}$ above sea level. The mean annual rainfall ranges from $800-850 \mathrm{~mm}$. The mean annual temperature ranges from $18-29^{\circ} \mathrm{C}$. During seed crop growth temperatures and rainfall were: (December 2015- June 2016) $31-21{ }^{\circ} \mathrm{C}$ and $100-0.8 \mathrm{~mm}$, respectively (annex 1). Chinhoyi is located in Mashonaland West province under agro

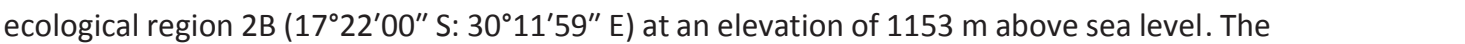
mean annual rainfall and temperature ranges from $700-800 \mathrm{~mm}$ and $22-29{ }^{\circ} \mathrm{C}$, respectively. During seed crop growth temperatures and rainfall ranged (December 2015- June 2016) from 31-22 ${ }^{\circ} \mathrm{C}$ and 73-0.31 mm, respectively (Annex 1), (authors, https://www.worldweatheronline.com Historical average weather 20/04/2017). 
Annex 2 Overview of TLV seed samples harvested and collected from farmers

\begin{tabular}{|c|c|c|c|c|c|}
\hline & Research & Farmer 4 & Farmer 1 & Farmer 2 & Farmer 3 \\
\hline $\begin{array}{l}\text { Farmer/Organizati } \\
\text { on }\end{array}$ & $\begin{array}{l}\text { organization } \\
\text { (Farmer 5) }\end{array}$ & & & & \\
\hline February, 2016 & Cleome gynandra & - & - & - & - \\
\hline March, 2016 & - & - & Cleome gynandra & - & - \\
\hline May, 2016 & - & - & $\begin{array}{l}\text { Amaranthus } \\
\text { hybridus }\end{array}$ & - & $\begin{array}{l}\text { Amaranthus } \\
\text { hybridus }\end{array}$ \\
\hline May, 2016 & - & - & Bidens pilosa & - & Bidens pilosa \\
\hline May, 2016 & - & - & - & - & Cleome gynandra \\
\hline June, 2016 & Bidens pilosa & Bidens pilosa & - & Bidens pilosa & - \\
\hline June, 2016 & $\begin{array}{l}\text { Amaranthus } \\
\text { hybridus }\end{array}$ & $\begin{array}{l}\text { Amaranthus } \\
\text { hybridus }\end{array}$ & - & $\begin{array}{l}\text { Amaranthus } \\
\text { hybridus }\end{array}$ & - \\
\hline June, 2016 & - & Cleome gynandra & - & Cleome gynandra & - \\
\hline
\end{tabular}

Note: - no harvests were carried out

Annex 3 Seedling evaluation intervals (ISTA, 2016)

TLV species

\begin{tabular}{|c|c|c|c|}
\hline $\begin{array}{l}\text { Seedling evaluation days after } \\
\text { planting }\end{array}$ & Bidens pilosa & Amaranthus hybridus & Cleome gynandra \\
\hline Three & + & - & - \\
\hline Seven & + & + & + \\
\hline Fourteen (final count) & + & - & - \\
\hline Fourteen & - & + & + \\
\hline Twenty one & - & + & + \\
\hline Twenty eight (final) & - & + & + \\
\hline
\end{tabular}

Note: + seedling evaluation day, - no seedling evaluation done 
Annex 4 Qualitative morphological characters of three species of traditional leafy vegetables

\begin{tabular}{|c|c|c|c|c|c|c|c|c|}
\hline $\begin{array}{l}\text { Seed } \\
\text { source }\end{array}$ & $\begin{array}{l}\text { Colour } \\
\text { stem }\end{array}$ & of & $\begin{array}{l}\text { Colour of } \\
\text { leaf }\end{array}$ & $\begin{array}{l}\text { Anthocyanin colouration } \\
\text { on leaf base }\end{array}$ & Leaf size & Shape of leaf & $\begin{array}{l}\text { Colour of near } \\
\text { ground stem }\end{array}$ & $\begin{array}{l}\text { Damping } \\
\text { off }\end{array}$ \\
\hline \multicolumn{9}{|c|}{ Amaranthus hybridus } \\
\hline Farmer 1 & $\begin{array}{l}\text { Green } \\
\text { purple }\end{array}$ & $\&$ & Light green & Absent & Broad & Ovate & Purple & None \\
\hline Farmer 2 & \multicolumn{2}{|c|}{ Light green } & Light green & Absent & Narrow & Lanceolate & Green & None \\
\hline Farmer 3 & \multicolumn{2}{|c|}{ Red purple } & $\begin{array}{l}\text { Green \& } \\
\text { purple }\end{array}$ & Absent & Broad & Ovate & Red purple & None \\
\hline Farmer 4 & $\begin{array}{l}\text { Green } \\
\text { purple }\end{array}$ & \& & Light green & Light purple & Broad & Ovate & Purple & Present \\
\hline Farmer 5 & $\begin{array}{l}\text { Green } \\
\text { purple }\end{array}$ & \& & Light green & Absent & Broad & Ovate & Purple & None \\
\hline \multicolumn{9}{|c|}{ Cleome gynandra } \\
\hline Farmer 1 & \multicolumn{2}{|c|}{ Light green } & Light & Absent & Broad & Ovate & Light green & None \\
\hline Farmer 2 & \multicolumn{2}{|c|}{ Light green } & Light & Absent & Broad & Ovate & Light green & None \\
\hline Farmer 3 & \multicolumn{2}{|c|}{ Light green } & Light & Absent & Broad & Ovate & Light green & None \\
\hline Farmer 4 & \multicolumn{2}{|c|}{ Light green } & Light & Absent & Broad & Ovate & Light green & Present \\
\hline Farmer 5 & \multicolumn{2}{|c|}{ Light green } & Light & Absent & Broad & Ovate & Light green & None \\
\hline \multicolumn{9}{|c|}{ Bidens pilosa } \\
\hline Farmer 1 & $\begin{array}{l}\text { Purple } \\
\text { green }\end{array}$ & $\&$ & Green & Absent & Intermediate & Oblanceolate & Red purple & None \\
\hline Farmer 2 & $\begin{array}{l}\text { Purple } \\
\text { green }\end{array}$ & $\&$ & Green & Absent & Intermediate & Oblanceolate & Red purple & None \\
\hline Farmer 3 & $\begin{array}{l}\text { Purple } \\
\text { green }\end{array}$ & $\&$ & Green & Absent & Intermediate & Oblanceolate & Red purple & None \\
\hline Farmer 4 & $\begin{array}{l}\text { Purple } \\
\text { green }\end{array}$ & $\&$ & Green & Absent & Intermediate & Oblanceolate & Red purple & None \\
\hline Farmer 5 & $\begin{array}{l}\text { Purple } \\
\text { green }\end{array}$ & $\&$ & Green & Absent & Intermediate & Oblanceolate & Red purple & None \\
\hline
\end{tabular}


Annex 5 The two-way interactions between media and time percentage germination of Amaranthus hybridus

\begin{tabular}{|c|c|}
\hline Media*Time & Mean \\
\hline Prechill+KNO ${ }_{3}$ August & $45(1.52 a)$ \\
\hline Prechill+KNO ${ }_{3}$ September & 51 (1.60a) \\
\hline Prechill+KNO ${ }_{3}$ October & 55 (1.67ab) \\
\hline Prechill+KNO ${ }_{3}$ November & 57 (1.72ab) \\
\hline Prechill+KNO ${ }_{3}$ February & 81 (1.91b) \\
\hline Field December & $36(1.60 a)$ \\
\hline Water February & 80 (1.90b) \\
\hline LSD 5\% & 0.25 \\
\hline
\end{tabular}

Annex 6 The two-way interactions between media and time percentage germination of Cleome gynandra

\begin{tabular}{|c|c|}
\hline Media*Time & Mean \\
\hline Prechill+KNO ${ }_{3}$ August & 0 (0.00a) \\
\hline Prechill+KNO ${ }_{3}$ October & $1(0.24 a b)$ \\
\hline Prechill+KNO ${ }_{3}$ September & 2 (0.31abc) \\
\hline Prechill+KNO ${ }_{3}$ November & $3(0.41 b c)$ \\
\hline Prechill+KNO${ }_{3}$ February & $11(0.66 \mathrm{~cd})$ \\
\hline Field December & 17 (0.95de) \\
\hline Pre-soak24hr February & 23 (1.33ef) \\
\hline Pre-soak48hr February & $24(1.36 f)$ \\
\hline LSD 5\% & 0.38 \\
\hline
\end{tabular}

Annex 7 The two-way interactions between media and time percentage germination of Bidens pilosa

$\begin{aligned} \text { Media*Time } & \text { Mean } \\ \text { Field December } & 76(1.89 \mathrm{a}) \\ \text { Water September } & 83 \text { (1.92ab) } \\ \text { Water August } & 84(1.93 \mathrm{ab}) \\ \text { Water October } & 87(1.94 \mathrm{~b}) \\ \text { Water November } & 89(1.95 \mathrm{~b}) \\ \text { Water February } & 91(1.96 \mathrm{~b}) \\ \text { LSD 5\% } & \mathbf{0 . 0 4}\end{aligned}$


4 Determinants of household demand for traditional leafy vegetables in Zimbabwe $^{3}$

3 This chapter is based on the article: Dube, P., Ihle, R. \& Heijman, W. J. M. (2018). Determinants of household demand for traditional leafy vegetables in Zimbabwe. To be submitted to Outlook on Agriculture. 


\section{Abstract}

Traditional leafy vegetables have a significant role in the traditional diets of many people in Zimbabwe, with over $75 \%$ of the population using them. For a targeted development of the traditional leafy vegetable sector, detailed knowledge on consumption and expenditure patterns for these vegetables is needed. We fill this gap by analysing a survey carried out in four districts of Zimbabwe. We evaluate the influence of various socio-economic household characteristics on the quantities they consume and the amounts they spent on traditional leafy vegetables. Based on a multivariate linear regression model, we find that households in poor districts consume and spend on average less on fresh traditional leafy vegetables although they consume them more being conserved as dried. Four traditional leafy vegetable species play a significant role in Zimbabwean diets but with varying regional preferences. A number of socio-economic households' characteristics such as years of schooling, healthiness and geographical location are found to determine consumption and expenditure. Seasonal availability is found to affect their prices and influences the frequency and amount of consumption. We suggest that the public sector might stimulate the demand for traditional leafy vegetables so that they are more incorporated into the daily menus of Zimbabweans. Such a promotion might be an inexpensive way to ensure nutrition security and support domestic farmers. This goal can be achieved through promotional campaigns on the healthiness of these vegetables, developing year-round production and improving the availability and quality of fresh and dried vegetables in retail markets. 


\subsection{Introduction}

Traditional leafy vegetables (TLVs) are plants which originate on the African continent and have a long history of cultivation and domestication to African conditions and use (AmbroseOji, 2009). Researchers are increasingly realising the importance of TLVs for improving food and nutrition security for households in urban and rural communities (Schippers, 2000). Notably, TLVs have been positively hypothesized to contribute significantly in the WHO global initiative to increase the consumption of vegetables in African countries (Smith \& Eyzaguirre, 2007). They are rich in minerals and micronutrients and have therefore a large potential for alleviating hunger and malnutrition in Africa. High levels of anaemia in women was observed on districts with higher calorie consumption (Benson, 2017), thereby showing limited potential for energy based food in addressing micronutrient deficiencies. In comparison, TLVs are more resilient to adverse weather conditions and unpredictable weather changes caused by climate change than staple crops and industrially bred-exotic vegetables, e.g. cabbage. As a result, TLVs can give important contributions during seasonal vegetable production gaps and towards food and nutrition insecurity reduction. They can also provide a 'safety net' during periods of shortage of other foods caused by crop failures.

Despite their potential to improve food and nutrition security (Schippers, 2000), their intake is relatively lower than the recommended dietary level in many African countries such as Zimbabwe. A minimum of $400 \mathrm{~g}$ per capita of fruits and vegetables per day is the dietary guideline for good health (Ruel et al., 2005). The per capita consumption of vegetables is $30 \mathrm{~g}$ in Zimbabwe (FAO, 2015), which is far below the recommended threshold for good health. Vegetable consumption, particularly TLVs, across Africa is influenced by many factors. These factors include product-related, socio-demographic, socio-economic and other consumerrelated characteristics (Weinberger \& Msuya, 2004; Ambrose-Oji, 2009; Odhav et al., 2007; Banwat et al., 2012). For instance, people consume large quantities of fresh TLVs (Banwat et al., 2012) compared to dried (Twine et al., 2003). Younger generations and households with a high income consume less (Odhav et al., 2007). FAO et al. (2012) also confirmed that a lack of information on the nutritional value of TLVs, the knowledge of how to prepare them and a lack of time to prepare them are some of the factors affecting their consumption. 
Most research on the determinants of food demand (e.g. Ruel et al., 2005; Solomon, 2009; Yen et al., 2011) leads to a better understanding of the reasons in favour of and against vegetable consumption. Socio-demographics (including education, age, ethnicity, income and location) were identified as predictors of vegetable consumption in Malaysia (Yen et al., 2011). Similarly, a positive relationship between income, gender or location and the consumption of vegetables was shown (Ruel et al., 2005). Attitudes towards food create variation in food consumption and at the same time influence the shopping and buying habits of consumers (Solomon, 2009). However, the determinants of household consumption of and expenditure on TLVs are not well investigated in Zimbabwe.

Considerable attention has been given to household food expenditures on vegetables in developed countries in particular and to a lesser extent on TLVs. For example, Oral \& Love (1983) investigated the determinants of household expenditure on fresh vegetables by USAmericans, Blisard et al. (2004) analysed the low-income households' expenditures on vegetables in the United States of America and Smallwood \& Blaylock (1984) explored the household expenditures on vegetables by US-Americans. In a previous analysis, household income, age and gender were found to have the highest influence on vegetable expenditure by US-Americans (Oral \& Love, 1983). Higher-income households spent more on healthy food compared to their lower-income household counterparts (Oral \& Love, 1983). Furthermore, low-income households purchase lower quality vegetables (Blisard et al., 2004). Other factors, like knowledge of nutrition, played a crucial role in determining household expenditure on vegetables (Smallwood \& Blaylock, 1984). This study is motivated by the need to gain a better understanding of how location, socio-demographic characteristics and socio-economic characteristics of the households and product-related characteristics affect household monthly expenditure on TLVs. This study is valuable since the literature on household monthly expenditure on TLVs is scanty.

This study relies on expenditure literature of other crops like exotic lettuce, spinach, cabbage, broccoli and asparagus, which are relatively different in terms of consumption and expenditure. Exotic crops already listed are relatively modernised, as they are regarded as Western crops and are more expensive than TLVs. Therefore, depending on such literature may give misleading impressions regarding household expenditure on TLVs to policy makers 
by overstating or understating the expected influence of these factors on expenditure. However, the determinants of household demand for TLVs are barely studied for Southern Africa. This study fills this gap by examining the determinants of household demand ${ }^{4}$ for TLVs using primary data from four districts of varying food security status in Zimbabwe. More specifically, the study addresses the following three objectives:

a. Examine the influence of socio-demographic and socio-economic characteristics of the households on the monthly household consumption of and expenditure on TLVs;

b. Explore the influence of product-related characteristics and location on the monthly household consumption of and expenditure on TLVs. The product-related characteristics are factors which include: healthiness, food supply constraints, convenience supply, cultural heritage, local loyalty and dried or fresh;

c. Understanding the consumers' preferences for TLVs.

The remaining sections of this paper is structured as follows: Section 2 presents the literature review. In section 3, we describe the methodology of the study. Section 4 reports the results. The final section discusses the findings and draws conclusions on the determinants of household consumption of and expenditure on TLVs in Zimbabwe.

4 We follow Smallwood \& Blaylock (1984) by measuring demand in terms of physical food consumption in quantity term (in kilograms) as well as monetary value terms (as expenditures in US\$). 


\subsection{Literature Review}

The focus of this analysis lies on the determinants of household consumption of and expenditure on TLVs. Food consumption is often measured in terms of quantity (by weight in kilograms) and money value (expenditure) in household surveys (Smallwood \& Blaylock, 1984). A very relatively weak relationship (0.09) was observed between household monthly consumption and expenditure; therefore, they were both included as dependent variables in the two models.

\section{Determinants of traditional leafy vegetable consumption}

Weinberger \& Msuya (2004) reported an inverse relationship between household income and wealth and TLV consumption. Poor people regularly consume TLVs, implying that they are probably regarded as food of inferior quality (Jansen Van Rensberg et al., 2007). In addition, the elite urban dwellers and middle-income earners consume little of it in fear of risking their health (World Vegetable Center, 2006). Preferences may vary per region. Mukwereza (2002), for example, reported spider flower, pumpkin and cowpea leaves as the most preferred TLVs in Zimbabwe.

In Zimbabwe, the production of TLVs is mainly during the rainy season. Consumption of dried TLVs mitigates food insecurity and vegetable seasonal gaps, particularly during the dry seasons. The processing of TLVs may slightly vary among the different consumer cultures (Jansen van Rensburg et al., 2004). The processing itself reduces the nutrient composition of TLVs (Mosha et al., 1997), which may in turn create the variations in consumption. Jansen van Rensburg et al. (2004) and Croft et al. (2014) reported that consumption of TLV types is influenced by location and cultural backgrounds.

This study hypothesizes that seasonality, product-related characteristics, socio-demographic household characteristics and socio-economic household characteristics influence TLV consumption. The socio-demographic and socio-economic household characteristics include the following: female decision-maker, age, household size, household income, household asset value, years of schooling, time to the preferred market and geographical location (Harare, Makonde, Mbire and Binga). The considered product-related characteristics are 
healthiness, food supply constraints, convenience of TLV supply, intrinsic preference, cultural heritage and local loyalty. Households with higher incomes regard TLVs as inferior food and a poor man's food (Jansen Van Rensberg et al., 2007) and tend to substitute the TLVs with exotic vegetables like cauliflower, broccoli and cabbage (Weinberger \& Msuya, 2004). A negative income elasticity of demand is associated with TLVs since it is most likely the food of lowerincome groups. Availability of TLVs in supermarkets and greengroceries and promotional campaigns on the health benefits associated with consuming TLVs (Irungu et al., 2008) are expected to change the negative perceptions among high-income, highly educated and elite households (Gido et al., 2017). Dunn et al. (2012) argued that more time to a preferred market constrains access to vegetables, leading to a less frequent and thereby smaller consumption of vegetables.

A female decision-maker is expected to lead to an increase in consumption of TLVs as females have a high interest in vegetable consumption. Females have good nutritional knowledge (Gido et al., 2017). Younger generations consume less TLVs because their consumption is associated with traditional lifestyles and out of fashion (Abukutsa, 2010). Bigger households need a larger quantity of food; as a result they consume cheap food like TLVs. A study by Gido et al. (2017) reported that TLV acceptance is constrained by location. Households in Harare have little interest in TLVs because they are modernized and urbanized and associate TLV consumption with poverty. Makonde has both urban and rural areas. There, consumption takes two sides. In rural areas, the consumption of TLVs can regarded as positive because people are poor and regard TLVs as food. For urban dwellers, the view on their consumption is opposite, i.e. negative. Mbire and Binga are typical rural and remote parts of the country; households are mostly poor and consider TLVs as an important staple. A study by Smallwood \& Blaylock (1984) showed that households in predominantly rural areas that are poor tend to regard vegetables as food.

Healthiness, food supply constraints, convenience of TLV supply, intrinsic preference, cultural heritage and local loyalty are product-related variables and are not hypothesized in literature (for full details see Annex Table A1). Healthiness is hypothesized to positively influence TLV consumption. If the consumers appreciate the nutritional, health and medicinal benefits associated with TLV consumption, they are expected to consume more. Food supply 
constraints is hypothesized to positively influence TLV consumption. People are expected to consume more if TLVs are the only vegetables growing in the area and the only vegetables at markets due to limited alternatives. With respect to convenience, if TLVs become readily available and are sold everywhere, consumers are expected to consume more TLVs. Baker \& Wardle (2003) reported that convenience is a determinant that typically influences vegetable consumption. Intrinsic preference is hypothesized to positively influence TLV consumption. If TLVs become increasingly important for people, particularly for their taste, it is more likely that more TLVs will be consumed. Cultural heritage positively influences TLV consumption. If a consumer appreciates their cultural heritage, they are expected to consume more TLVs. Local loyalty is hypothesized to positively influence TLV consumption. If TLVs are cheap and locally produced, a higher consumption of TLVs is expected.

\section{Determinants of traditional leafy vegetable expenditure}

Food consumption can be quantified in monetary value terms (expenditure) in household surveys (Smallwood \& Blaylock, 1984). In this paper, we use both consumption and expenditure. Literature on household expenditure on TLVs was difficult to come across; therefore we used literature for other vegetables (cabbages, lettuce, carrots). Moreover, we had challenges in getting literature on household expenditure on vegetables specifically for Zimbabwe and even other African countries. Instead we relied on American literature. Considering the fact that the two countries are totally different in terms of socio-demographic and socio-economic characteristics of households, this decision gave rise to problems of interpretation with regard to the literature's hypotheses and results. A study by Oral \& Love (1983) showed that household income, age and gender influence vegetable expenditure in the United States of America. We therefore hypothesize that household income, age and gender positively influence expenditure on TLVs.

A female decision-maker is expected to spend more on TLVs as they are responsible for shopping. Studies by Oral \& Love (1983) as well as Smallwood \& Blaylock (1984) showed that expenditure levels generally increase with age. Since these authors used exotic crops in the United States of America, these results can be interpreted differently with regard to TLVs. TLVs are regarded as food for poor people (Jansen Van Rensberg et al., 2007). Smallwood \& Blaylock (1984) showed that bigger households buy cheap food. Blisard et al. (2004) on 
income elasticity of demand reported that an increase in vegetable expenditures are rapid as income rises, but saturation levels are approached at relatively low levels of income. Persons with more years of schooling regard TLVs as inferior food and will spend less on these products. However, with an increased awareness of the health and nutritional benefits associated with TLVs, people are more likely to change their negative perceptions towards TLVs and thereby more likely to spend more. A study in Tanzania shows that during the rainy season the lower-income households hardly buy TLVs since they collect them from the forest or produce them in their own gardens (Weinberger \& Msuya, 2004). Whereas, the higherincome groups may prefer to buy their TLVs from supermarkets, which is more expensive. Households taking more time and money to travel to vegetable markets constrains expenditure. In other words, they would spend less on TLVs.

Differences in locations may lead to differences in purchasing power. Harare is a reference category. It is the national capital, so people living in Harare are expected to spend more on TLVs. This can be partly attributed to an increased awareness of the health and nutritional benefits associated with TLVs and higher prices of TLVs in supermarkets resulting in greater expenditure. Makonde is a nonmetropolitan area, such that the people in Makonde have a lower purchasing power than those in Harare. Whereas Mbire and Binga are nonmetropolitan areas and typical rural and remote parts of the country. Hence people in these areas also have a lower purchasing power than those living in Harare. With regard to healthiness, if people embrace nutrition and thereby the health benefits of TLVs, they are expected to spend more on these products. If other food supplies are constrained, people are more likely to spend more on TLVs. If TLVs are available locally, at supermarkets and sold everywhere, consumers would spend more. If people would appreciate TLVs taste, easy to prepare and increasingly recognize them important as food, are more likely to spend more. If people would embrace traditionalism attached to TLV consumption, they are expected to spend more. If people would appreciate that TLVs are fairly cheap compared to other vegetables and increasingly important for their health, they are more likely to spend more. Smallwood \& Blaylock (1984) showed that there are lower expenditures on dried vegetables.

A deeper understanding of factors that influence their consumption and household expenditure can enable the development of tools to increase their production and 
consumption as well as consequently improve food security for and the diet quality of the Zimbabwean people. Also understanding locational difference, seasonality influence and consumers' preferences and attitudes helps policy makers and people involved in the TLV supply chain be better positioned to timely target specific niches in the market. For example, a TLV farmer can be better positioned for investing in a greenhouse in order to be able to supply the fresh vegetables to markets throughout the year. Furthermore, such information is useful to breeders in case they want to improve the quality of seed to be more homogenous. They are also equipped with knowledge on which TLV species are suitable for a certain area. Researchers can use this knowledge to improve the germination of these TLV seeds in order to break the seasonality. Also, gathering detailed empirical information is a pre-condition for the development of appropriate policy measures for stimulating production and consumption of TLVs in Zimbabwe and across Sub-Saharan Africa in general. It is worth noting that the consumption of TLVs is crucial for the Zimbabweans as these vegetables may grow naturally and have high nutritional value. Furthermore, TLVs can serve as a means to increase food and nutrition security for many households that experience financial difficulties, which is a common situation in Zimbabwe.

Consumption of TLVs across age groups has a good potential to improve nutrition, thereby addressing malnutrition problems faced by the country (UN, 2010). TLVs are mostly cooked and consumed accompanying staples ${ }^{5}$. Maize is the staple food in Zimbabwe; however, it is generally low in micronutrients. Micronutrient deficiencies are often referred to as hidden hunger because they can occur within the context of adequate energy intake and pose a key developmental challenge (FAO et al., 2012). Nevertheless, Zimbabwe has a high HIV/AIDS rate, with the prevailing rate at $14.7 \%$ (UN, 2010). Consumption of TLVs can be therefore important to alleviate malnutrition, improve nutrition and help alleviate HIV/AIDS (Yang \& Keding, 2009) as a medicinal supplement therapy for fighting HIV/AIDS (Friis, 2006). TLVs are excellent sources of vitamins A and C, iron, protein, minerals as well as fibre (Oluoch et al., 2009) and can therefore be important for people without access to meat or other sources of protein (Schippers, 2000).

\footnotetext{
${ }^{5}$ Staples is a thick porridge made by mixing boiling water with maize or sorghum or millet flour.
} 


\subsection{Methodology and Data Analysis}

\section{Study area and sampling strategy}

Figure 5 shows the spatial distribution of food insecure rural populations among the districts of Zimbabwe as well as the geographical location of the study areas. Most of the districts show high levels of food insecurity; that is, 10 to 50 percent of the rural population is food insecure. In the north of the country and around Harare the food situation is best. The largest problems exist in Binga, Mbire, Chivi and Beitbridge. The south of the Makonde district is food insecure, whereas its north, where we conducted the survey, is moderately food secure.

The survey was conducted in the districts of Binga, Mbire, Makonde and Harare. In the first stage, the districts were purposively selected to represent areas that are either severely food insecure or highly food secure (Figure 5). The Binga district is highly and perennially food insecure, followed by the Mbire district, whereas Harare and the selected part of Makonde are highly food secure. Binga is characterised by highly erratic and low rainfall (less than 450 $\mathrm{mm}$ /year) and poor soil. Mbire receives $450-650 \mathrm{~mm}$ of rainfall per year with frequent seasonal droughts and severe dry spells during the rainy season. In Harare and Makonde, rainfall ranges from 750 to $1000 \mathrm{~mm} /$ year and is very reliable. Soil in both districts are of good quality and therefore suitable for intensive cropping and livestock production (Vincent and Thomas, 1961). These districts are diverse in terms of the socio-demographic and socioeconomic characteristics of households and therefore offer interesting contrasting cases to analyse and compare (for full details of the socio-demographic data of the districts surveyed see Annex 3). 
Figure 5 Rural food insecurity in Zimbabwe and the surveyed areas

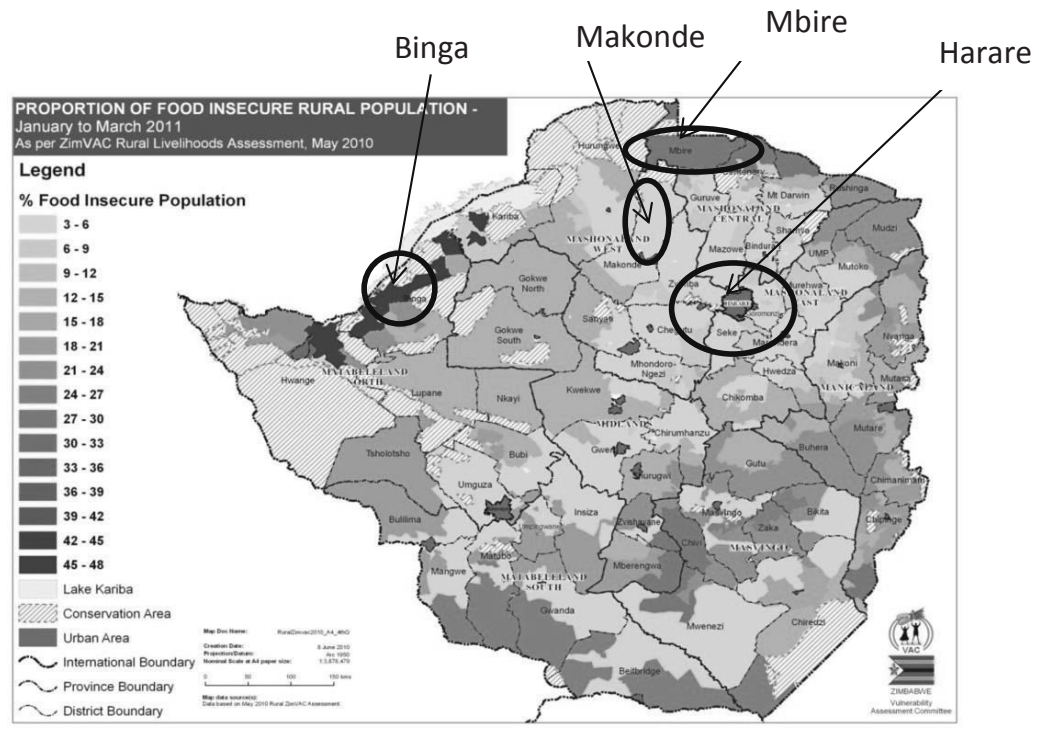

Source: FAO (2010).

The study was conducted in two phases. The first phase was conducted in August and

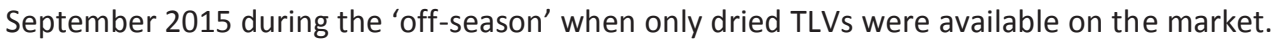
The second phase was conducted in October 2015 to January 2016 duringetmsoton when fresh TLVs were available on the market. The seasonality differences also offered interesting contrasting cases to analyse and compare. In the second stage, the sample was drawn using a multistage sampling procedure, stratified into rural and urbanized regions, which later contain the national capital, Chinhoyi-Makonde and growth points and sectors in the districts (for full details see Annex 5). Respondents were interviewed to obtain information regarding the gender of the head of their household, the relationship of the head of their household to the respondent, marital status, education level, occupation, income, asset value, household size, purchasing habits, attitude, purchasing preferences, gender of the decision-maker on the type of vegetables the household eats, preferred markets, household monthly expenditure on TLVs, frequency of consumption and quantity per each consumption. Solomon (2009) refers to an attitude in marketing terms; it is defined as a general evaluation of a product or service formed over time. An attitude satisfies a personal motive and at the same time affects the shopping and buying habits of the consumer. In this study, the term attitude is used to capture factors that constrain the shopping and buying 
habits of respondents in relation to TLVs, for example "I buy TLVs if they are the only vegetables on the market". Other attitudes towards TLVs that were rated by respondents included: healthiness, food supply constraints, convenience of supply, cultural heritage and local loyalty (for complete details see Table Annex 1).

In total the survey had 1000 adult respondents. In each district 250 respondents were interviewed, specifically 125 respondents on the first round ('off-season') and 125 respondents on the second round ('on-season'). We conducted the surveys in AugustSeptember 2015 and October 2015 to January 2016 for the first and second round, respectively. Data was collected using a structured questionnaire pre-tested on both rural and urban areas in March-June 2015. The questionnaire contained a section with 33 socioeconomic questions (ethnicity, household income, wealth, etc.) and another section asking 27 questions on TLV purchasing habits (for complete details see Annex Questionnaire for Chapter 4). Most of the questions were closed, while some responses were coded using a Likert scale from 1 to 5 with 1 for strongly decrease/very unimportant/completely disagree/never and 5 strongly for increase/very important/completely agree/very frequently.

\section{Empirical framework}

This study incorporates socio-demographic, socio-economic and product-related aspects, such that the consumption of TLVs can be explained by:

$$
C=\sum_{i=1}^{6} \beta_{i} X_{r i}+\sum_{i=1}^{6} \propto_{i} X_{p i}+\sum_{i=1}^{5} \partial_{i} D_{i}+D_{2}\left[\sum_{i=1}^{6} \alpha_{i} X_{r i}+\sum_{i=1}^{6} \gamma_{i} X_{p i}+\sum_{i=1}^{4} \rho_{i} D_{i}\right]+\varepsilon_{i}
$$

The variable $C$ denotes consumption (in $\mathrm{kg} /$ household per month). $X_{r i}$ is an independent variable that describes the respondents' demographic and socio-economic characteristics, which includes the gender of the decision-maker regarding the type of vegetables the household consumes, age, household size, household monthly income, household asset value, approximate years of schooling and approximate time to the preferred market. $X_{p i}$ denotes the explanatory variable relating to the product characteristics, including healthiness, food supply constraints, convenience supply, intrinsic preference, cultural 
heritage and local loyalty. $D_{i}$ is a dummy variable that describes the female decision-maker on the type of vegetable the household eats, location or season. Therefore, $D_{1}=1$ if the female is decision-maker variable and equals 0 otherwise. $D_{2}=1$ if the season is off and equals 0 otherwise. $D_{3}=1$ if the location of the respondent is Makonde and equals 0 otherwise. $D_{4}=1$ if the location of the respondent is Mbire and equals 0 otherwise. $D_{5}=1$ if the location of the respondent is Binga and equals 0 otherwise. $\varepsilon_{i}$ is the error term. Likewise, the consumption of TLVs is influenced by the already stated variables expressing seasonality, i.e. off or onseason.

Furthermore, this study measures and explains household expenditure. The amount spent per household per month on TLVs based on the variables introduced in section 2 can be expressed as:

$E=\sum_{i=1}^{6} \beta_{i} X_{r i}+\sum_{i=1}^{6} \propto_{i} X_{p i}+\sum_{i=1}^{5} \partial_{i} D_{i}+D_{2}\left[\sum_{i=1}^{6} \alpha_{i} X_{r i}+\sum_{i=1}^{6} \gamma_{i} X_{p i}+\sum_{i=1}^{4} \rho_{i} D_{i}\right]+\varepsilon_{i}$

The variable $E$ denotes expenditure (in US\$/household per month). The following independent qualitative and quantitative variables were included in the model: female decision-maker on the type of vegetables the household consumes, age, household size, household monthly income, household asset value, years of schooling, time to the preferred market, healthiness, food supply constraints, convenience of TLV supply, intrinsic preference, cultural heritage and local loyalty. Similar to the expression of consumption, the interactions between each variable and the dummy denoting 'off-season' is included. The reasons of using each of the explanatory variables in both models are presented in Table Annex 4.

To further explore the effect of consumer attitudes on TLV consumption and expenditure, 16 items, which were rated by consumers based on a Likert scale ranging from completely disagree (1) to completely agree (5), were used. Thereafter the factor analysis of the 16 items were resolved to six factors with Eigenvalues greater than 0.4. The items classified under healthiness include medicinal properties, beneficial properties, nutritious, high quality food and improve family health. The variable food supply constraints was defined by two items, namely only vegetables growing in my area and only vegetables on the market. Also, the items 
under convenience of TLV supply include sold everywhere and readily available, whereas intrinsic preference includes like their taste, not exotic and easy to prepare. For cultural heritage and local loyalty, these items were traditional and reminds me of rural areas as well as cheap and locally produced, respectively (for full details see Table Annex 1).

One dummy variable was also included to indicate gender of the decision-maker on the type of vegetable the household eats. Harare, Makonde, Mbire and Binga are the geographical location. Three dummy variables were included to indicate the location, specifically, Makonde, Mbire and Binga. We had one dummy variable to represent the season (off-season or dried TLVs). Monthly household income was defined by nine categories: 100 USD, 199 USD, 350 USD, 450 USD, 495 USD, 800 USD, 1000 USD, and 1001 USD for above 1000 USD. We used the upper limit of each category as the category's title. The total household asset value was calculated after getting the average price for each asset per ten Zimbabwean provinces. We did not include prices within affluent suburbs in cities like Harare's Borrowdale or the price of expensive cars like Benz. As such our household asset price was calculated basing minimal prices obtained from the ten provinces. 


\subsection{Results}

\section{Sample characteristics}

First, we carried a frequency analysis for the variables that we intended to include in the analysis. The results are presented in table form (Table 13-Table 15). After deleting incomplete questionnaires, 933 respondents were included in the analysis. They were predominantly household heads (57.4\%) and spouses (31.6\%), with a mean age of 40.9 years and a mean of 11.24 years of schooling, which is equivalent to a secondary school level. The household heads and spouses were responsible for the decision making on shopping, the amount spent on vegetables, the household diet and the type of vegetables the household ate. For the gender of the decision-maker on the type of vegetable the household eats, $14.7 \%$ were males and $85.3 \%$ were females.

Table 13 shows descriptive statistics of selected key variables depending on the season. Averages for on- and off-season differ slightly. The average household monthly expenditure on TLVs is 8.81 USD on-season and 10.51 USD off-season, and each household consumes on average $4.14 \mathrm{~kg}$ of fresh TLVs and $1.15 \mathrm{~kg}$ of dried TLVs per month. Using an analysis of variance to determine if the mean monthly consumption and expenditure differ between the two seasons, we found a statistical significant difference in the mean monthly consumption and expenditure between the two seasons, $p=0.000$ and $p=0.006(p<0.05)$ respectively. Mean household monthly income is 388 USD and 405 USD for on-season and off-season, respectively. Whereas, the overall mean household income is 397 USD, relatively lower than the poverty line of 495 USD (July, 2015). The poverty line (PDL for July at 495 USD) represents the cost of a given standard of living that must be attained if a person is deemed not to be poor. The overall mean household size is 5 persons.

\begin{tabular}{|c|c|c|c|}
\hline Variable & On-season $(N=469)$ & Off-season $(N=464)$ & Overall ( $N=933)$ \\
\hline Mean monthly consumption (kg/hh) & 4.14 & 1.15 & 2.65 \\
\hline Mean monthly expenditure on TLVs (USD) & 8.81 & 10.51 & 9.66 \\
\hline Household size (number) & 5.31 & 5.53 & 5.32 \\
\hline Household assets value (USD) & 14324 & 15845 & 15084.5 \\
\hline Household income (USD) & 388 & 405 & 396.5 \\
\hline Age (in years) & 40.95 & 40.86 & 40.91 \\
\hline Number of years of schooling & 11.18 & 11.30 & 11.24 \\
\hline
\end{tabular}


Source: Authors.

Table 14 shows the mean monthly consumption and expenditure of TLVs per household by district and season. The mean monthly consumption of TLVs per household is larger for fresh than dried TLVs across all the districts. Regarding the consumption trends of dried TLVs in $\mathrm{kg} /$ household by district, a strong difference between Harare and Makonde at the one side and Mbire and Binga on the other becomes apparent. In the two poorest districts, Mbire and Binga, people consumed more dried TLVs. Harare, by far, exhibits a higher amount spent on TLVs for all seasons compared to the remaining three districts. Households in Makonde and Mbire spent the least on fresh TLVs per month compared to Harare and Binga. Across all the districts, people consumed and spent more than the median for both seasons.

Table 14 Mean monthly consumption of and expenditure on TLVs per household by district and season

\begin{tabular}{|c|c|c|c|c|c|c|c|c|}
\hline \multirow[t]{2}{*}{ Variable } & \multicolumn{2}{|l|}{ Harare } & \multicolumn{2}{|c|}{ Makonde } & \multicolumn{2}{|l|}{ Mbire } & \multicolumn{2}{|l|}{ Binga } \\
\hline & On & Off & On & Off & On & Off & On & Off \\
\hline$N$ & 119 & 115 & 112 & 109 & 116 & 116 & 122 & 124 \\
\hline Mean monthly consumption (kg/hh) & 4.81 & 0.78 & 4.26 & 0.79 & 3.58 & 1.50 & 3.89 & 1.46 \\
\hline Median & 3.0 & 0.24 & 3.0 & 0.48 & 1.5 & 0.48 & 1.5 & 0.48 \\
\hline Mean monthly expenditure on TLVs (USD) & 14.05 & 13.66 & 5.95 & 10.48 & 6.12 & 10.47 & 8.89 & 7.65 \\
\hline Median & 12.00 & 12.00 & 4.00 & 8.00 & 4.00 & 8.00 & 8.00 & 6.00 \\
\hline CV & 64.33 & 74.75 & 81.45 & 112.87 & 89.39 & 81.60 & 125.41 & 106.12 \\
\hline
\end{tabular}

Source: Authors.

Table 15 shows the descriptive analysis of socio-economic characteristics of the sample by district. Mean monthly income per household and household asset value is largest in Harare and lowest in Binga. Household respondents in Harare are younger with higher levels of education. Binga is the poorest district with the least household size. The result also exhibits the least number of years of schooling in Mbire and Binga.

Table 15 Descriptive analysis of socio-economic characteristics of the sample by district

$\begin{array}{lllll}\text { Variable } & \text { Harare } & \text { Makonde } & \text { Mbire } & \text { Binga } \\ N & 234 & 221 & 232 & 246 \\ \text { Household size (number) } & 5.27 & 6.14 & 5.20 & 5.12 \\ \text { Household assets value (USD) } & 31083.28 & 16257.46 & 7831.41 & 5639.62 \\ \text { Household income (USD) } & 670.61 & 410.67 & 279.51 & 236.05 \\ \text { Age } & 39.02 & 42.44 & 43.25 & 39.11 \\ \text { Number of years of schooling } & 14.33 & 11.83 & 9.63 & 9.28\end{array}$


Source: Authors.

Determinants of purchasing and consuming TLVS

To provide policy recommendations that promote the consumption of TLVs and to help address micronutrient deficiency problems in Zimbabwe, it is imperative to have a clear understanding of the determinants that have the most influence on or rather are the most important factors regarding TLV purchasing and consumption by households.

Hence, consumers were asked to rate the TLVs characteristics based on their preferences for both the TLV type and market to obtain TLVs. The rating was based on a Likert scale ranging from completely disagree (1 ) to completely agree (5 ). The definition of the "most important factor" was defined by the number of consumers responding to the top scale levels, i.e. agree and strongly agree (4-5).

Table 16 shows the frequency of TLV consumption per household by district and season. It also shows the preferences and preferred markets when purchasing TLVs. The top panel of the table depicts the number of households sampled. Below, frequencies of TLV consumption are shown. An analysis of variance shows that the frequency of consumption differs between the two seasons and districts $(p<0.001)$. Higher frequencies are observed on fresh TLVs across all the districts. Only a few respondents did not consume TLVs.

The consumer preferences regarding the top four TLV species are presented on the third panel of Table 16. Spider flower was the most preferred TLV in both seasons, followed by pumpkin leaves. The table emphasizes the pronounced differences between TLV consumption preferences across the regions of Zimbabwe as consumers in all surveyed regions prefer different TLVs. A similar variability is observed concerning the marketing channels most preferred by consumers (last panel of Table 16). In the capital, the Mbare Musika farmers' market is the most important source of TLVs, followed by supermarkets. In Makonde, supermarkets and farmer markets are still important, although subsistence production accounts for one third to a half of the respondents. In the remote rural regions, three quarters of the TLVs consumed are produced by the households themselves. Mbare Musika is one of the biggest markets in Harare. 
Similarly, to determine the most important preferred market channel to obtain TLVs, consumers were given a list of existing market channels, including markets in their respective areas, and asked to choose their most preferred market channel. The results are shown in the last panel of Table 16. Growing TLVs in their fields is much more important during all seasons for respondents living in the districts of Makonde, Mbire and Binga, where approximately half of all households grow them. The households in Mbire and Binga hardly purchase TLVs from the supermarkets. As could be expected, in Harare most respondents purchased the TLVs from Mbare Musika and supermarkets.

Table 16 Frequency of consumption and consumer attitudes, preferences and preferred markets when purchasing TLVs per household by district and season

Variable
Season
At most twice per month
Less than once every day
At least once every day
Other (not consuming/less than once per
month)

On Off On Off On Off On Off Overall Overall

On Off

$\begin{array}{llllllllll}119 & 115 & 112 & 109 & 116 & 116 & 122 & 124 & 469 & 464\end{array}$

Frequency of consumption (\% respondents)

$\begin{array}{llllllllll}10 & 33 & 6 & 22 & 1 & 29 & 3 & 16 & 5 & 25 \\ 68 & 55 & 80 & 59 & 57 & 45 & 24 & 50 & 57 & 52 \\ 21 & 7 & 13 & 9 & 39 & 20 & 73 & 23 & 36 & 15 \\ 1 & 5 & 1 & 10 & 3 & 6 & 1 & 11 & 2 & 8\end{array}$

TLV preferences (\% respondents)

$\begin{array}{lllllllllll}\text { Spider flower } & 31 & 40 & 36 & 29 & 42 & 34 & 51 & 52 & 40 & 39 \\ \text { Pumpkin leaves } & 33 & 30 & 29 & 28 & 25 & 37 & 24 & 20 & 28 & 29 \\ \text { Cowpea leaves } & 14 & 10 & 19 & 18 & 16 & 19 & 13 & 8 & 16 & 14 \\ \text { Okra } & 15 & 11 & 11 & 11 & 16 & 9 & 12 & 8 & 14 & 10 \\ \text { Other (25 species) } & 7 & 9 & 5 & 14 & 1 & 1 & 0 & 12 & 2 & 8 \\ & \text { Market preferences (\% respondents) } & & & & & \\ \text { Mbare Musika farmer market and retail } & 47 & 54 & 1 & 2 & 0 & 0 & 0 & 1 & 10 & 12 \\ \text { Supermarket } & 26 & 26 & 10 & 7 & 10 & 0 & 1 & 1 & 9 & 9 \\ \text { Farmers' market } & 6 & 7 & 33 & 25 & 6 & 3 & 1 & 2 & 11 & 9 \\ \text { Produced in own garden } & 8 & 4 & 31 & 49 & 72 & 77 & 71 & 77 & 28 & 52 \\ \text { Other (6 others) } & 13 & 9 & 28 & 17 & 12 & 20 & 28 & 19 & 42 & 18\end{array}$

Source: Authors. 


\section{Determinants of TLVs household consumption}

The study reports findings from 933 observations with $R^{2}=0.209$ and an overall $p$-value $<0.05$ of the model. Econometric results (for coefficients and full details see Table Annex 2) show that only household size significantly and positively influenced the average consumption of TLVs per month. As expected, an increase in household size by one resulted in an increased consumption of TLVs by $0.17 \mathrm{~kg}$ per month. However, female decision-maker and age have no effect on TLV consumption. Years of schooling has a negative influence on TLV consumption. As expected, the mean monthly consumption of TLVs per household is lower by $0.21 \mathrm{~kg}$ for every additional year of schooling of the respondent. However, the remaining socio-economic variables (household monthly income, household asset value and approximate time to the preferred market) have no influence on TLV consumption.

Against our expectations, all geographical locations, namely Makonde, Mbire and Binga, have negative coefficients, but the coefficient is only significant for Mbire. For the respondents who live in Mbire, the average monthly household TLV consumption is $1.44 \mathrm{~kg}$ and significantly lower than those in Harare. All product-related variables are hypothesized to positively influence TLV consumption. However, healthiness, convenience supply and intrinsic preference have positive coefficients; the remainder have negative coefficients. If healthiness increases by 1 unit, the mean TLV consumption per household increases by $0.40 \mathrm{~kg}$. If the food supply constraints increase by 1 unit, the mean consumption of TLVs per household decreases by $0.50 \mathrm{~kg}$, against expectations. The mean monthly household consumption of TLVs decreases by $0.48 \mathrm{~kg}$ for every unit change in local loyalty. The coefficient shown in Table Annex 2 is an estimate of the mean dried (off-season) TLV consumption in $\mathrm{kg} / \mathrm{hh}$, which is 5.68 as expected. The mean monthly consumption per household for the reference category is $6.33 \mathrm{~kg}$, compared to off-season value of $5.68 \mathrm{~kg}$. On average, each household consumes smaller quantities of dried TLVs than fresh.

Interactions between off-season and all the variables are not hypothesized. However, the interactions between age, years of schooling, Mbire, and food supply constraints and the offseason dummy have positive and significant coefficients. The remaining interactions are not significant, but they carry different coefficient signs. Age exhibits a positive interaction with off-season, specifically the consumption of TLVs increases by $0.04 \mathrm{~kg}$ per month per 
household. Mean household monthly consumption of TLVs is $0.18 \mathrm{~kg}$ for years of schooling interaction. During off-season, on average households in Mbire consume $1.81 \mathrm{~kg}$ TLVs per month, relatively larger than Harare. The mean monthly consumption of TLVs per household is increased by $0.68 \mathrm{~kg}$ if the TLVs are the only vegetables growing in the area and the only ones available on the market.

\section{Determinants of TLV household expenditure}

For household monthly expenditure on TLVs, our study used 933 observations with statistical calculations $\mathrm{R}^{2}=0.200$ and an overall $p$-value $<0.05$ of the model. The coefficients of the mean household expenditure on TLVs can be seen in Table Annex 2 in full detail.

All socio-demographic variables have no significant influence on household monthly expenditure on TLVs. Although, the female decision-maker is responsible for choosing the type of vegetable consumed by the household and buying the vegetables; however, with a coefficient of 0.36 USD the results show that the female decision-maker does not significantly influence household expenditure on TLVs. The coefficient of the age of respondent is negative and, against expectations, not significant. Bigger households are hypothesized to spend less on TLVs.

Interestingly, all the socio-economic variables included in the analysis have positive and significant influence on TLV expenditure. If the household monthly income increases by 100 USD, the mean monthly household expenditure increases by 0.40 USD. However, the effect of household asset, though significant, is very minimal, <0.01 USD for every 1 USD increase. For every additional year of schooling, the amount spent on TLVs increases by 0.22 USD. Against expectations, time taken to the preferred market positively and significantly influences the mean monthly expenditure. With a 60-minute change in time taken to the preferred market, the mean monthly expenditure increases by 0.04 USD.

The coefficients for all locational variables are negative, as expected. However, only the coefficients for Makonde and Mbire are significant. As a nonmetropolitan area, Makonde has a lower purchasing power than Harare, from which it is approximately $150 \mathrm{~km}$. Households in Makonde spent US\$6.82 lower on average per month than do their counterparts in Harare. 
Mbire is also a nonmetropolitan area and has, as is typical to the rural and remote parts of the country, a lower purchasing power than Harare. Households in Mbire spent 4.60 USD lower on average per month than do their counterparts in Harare. Although Binga spends lower than Harare and the coefficient is 2.43 USD, the poor district is not significantly different from the capital.

Smallwood \& Blaylock (1984) have observed significantly lower expenditures on dried vegetables. In this study, the results show a negative, although not significant, coefficient (4.23) on expenditure during off-season, as expected. For all product-related variables, only convenience supply and intrinsic preference have positively and significantly influence expenditure on TLVs. If the TLV convenience supply increases by 1 unit, the average monthly household expenditure increases by 1.24 USD. Similarly, if intrinsic preference increases by 1 unit, the average household monthly expenditure on TLVs increases by 0.89 USD.

Similarly, the interactions between off-season and all the variables are not hypothesized. Of all the interactions included in the model, only Makonde*off-season, Mbire*off-season and healthiness*off-season are positive and significant. The interactions improve the purchasing power on TLVs by, 5.70 USD, 5.88 USD and 1.30 USD, respectively. The interaction with female decision-maker*off-season, age*off-season, years of schooling*off-season and Binga*offseason is positive although not significant, and the remaining interactions have negative coefficients, but are not significant. 


\subsection{Discussion}

The study was conducted in four districts that differ in terms of food security, sociodemographic and socio-economic characteristics of households. Generally, we found that households in districts with low household monthly income consume and spend less on TLVs than those in districts with high household monthly income, e.g. Harare. We found that in two poor districts (Mbire and Binga) food insecure households predominantly consume more dried TLVs than their counterparts. The results also show that seasonal availability affects their prices and influences the frequency and amount of consumption. TLV preferences depend on location.

\section{Determinants of TLV household consumption explanations}

We predicted that female decision-makers on the type of vegetable the household consumes have a positive influence on the average monthly household consumption of TLVs. Females have a high interest in consuming vegetables because they have better nutritional knowledge. Following this logic, it is surprising that consumption of TLVs is not influenced by the gender of the household decision-maker, although the coefficient is positive. Previous studies by Baker \& Wardle (2003) clearly show that more women than men consume more vegetables because women are informed about the health benefits attached to vegetables. Related findings by Benson (2017) observed on average higher levels of malnutrition on districts where women were often excluded from household decisions. The reason for this anomaly can be related to promotional campaigns on the healthiness associated with consuming TLVs. Due to these campaigns, male decision-makers gain knowledge on the importance of TLVs in their household diets. Against expectation, the coefficient of age of the respondent is negative $(0.02 \mathrm{~kg})$. The older the respondent, the more consumption of TLVs. Since older people are custodians of knowledge, knowing recipes for TLVs results in a higher consumption of TLVs. All socio-economic variables were hypothesized to negatively influence TLV consumption, but only years of schooling is significant. Household monthly income has negligible, negative impact on TLV consumption.

Average monthly consumption of TLVs for Mbire differs significantly from that in Harare, the capital. Against expectations, TLV consumption is lower. Mbire is a typical rural and remote 
part of the country. There, households are poor and were predicted to consider TLVs as food. The results are possibly due to differences in the availability of TLVs on markets. A higher consumption of TLVs in Harare may be due to the availability of TLVs on urban markets, particularly in affluent supermarkets, and campaign programs explaining widely known nutritional benefits associated with TLV consumption. Irungu et al. (2008) found similar results. They reported that the availability of TLVs in affluent markets such as supermarkets increased their consumption in urban areas.

The consumers in western Kenya valued TLVs for their taste, nutritional and medicinal benefits (Abukutsa-Onyango, 2007). In this study, we hypothesized that all product-related characteristics would positively influence TLV consumption. Our results show that the coefficient for healthiness is positive and statistically significant as predicted; however, the coefficients for food supply constraints and local loyalty are negative and statistically significant, against our expectations. This finding indicates that people have a great interest in nutritious and healthy foods. This preference is also shown by the larger coefficients for all significant product-related variables, namely healthiness, food supply constraints, local loyalty and season, compared to socio-economic variables age and years of schooling. Our finding regarding the negative effect of food supply constraints suggests that being the only vegetables on the market as well as grown in the area does not mean people will consume more; they may consume other foods such as meat or cabbages.

\section{Determinants of TLV household expenditure explanations}

Most noteworthy is that all the coefficients for the socio-economic variables included in the model are positive and statistically significant in explaining the variations in household expenditure on TLVs. Household monthly income had a significant, positive influence on monthly expenditure on TLVs. Households with a higher-income spend more on TLVs than lower-income households do. Probably, higher-income households obtain their TLVs from supermarkets, greengroceries or organized markets. It is, therefore, not surprising that monthly expenditures on TLVs are higher for higher-income households. Weinberger \& Msuya (2004) reported that the consumers purchasing TLVs in supermarkets are willing to pay more as compared to consumers who purchase TLVs in local markets. We had hypothesized that all the socio-economic variables would negatively affect household 
monthly expenditure on TLVs. Persons with more years of schooling spend more on TLVs than those with less years of schooling do. Persons that have an increased awareness of the health and nutritional benefits associated with TLVs spend more on TLVs.

Households in Makonde and Mbire spent less on TLVs than in Harare. These findings are consistent with our hypotheses since these two districts are non-metropolitan areas. In other words, they have a lower purchasing power than Harare, the national capital. Hence, households in these two districts preferred to grow TLVs in their gardens, as shown in the last panel of Table 16. On the other side, Binga is a non-metropolitan area and a typical rural and remote part of the country, which has a lower purchasing power than Harare and has been hypothesized to spend less than Harare. The degree of urbanization may affect expenditure as the households located in rural areas may have opportunities for home gardens. On the other hand, regional differences may show differences in the general availability of TLVs. Household monthly expenditure on TLVs for people in Binga is not statistically significantly different from that in Harare. This may be a function of higher prices in Binga. Hassan et al. (2012) reported higher retail prices of vegetables in rural markets than in urban markets. Binga is predominantly rural, characterised by highly erratic and low rainfalls (less than 450 $\mathrm{mm}$ /year) and poor soil. For that reason, people may not have enough irrigation water to produce their own vegetables even though they wish to do so. Moreover, although we did not ask the price of TLVs in Binga, we were surprised to see a head of cabbage cost 2 USD when the same commodity is sold in Harare for 0.50 USD. Probably the price is higher for TLVs in Binga than in other districts.

Convenience supply and intrinsic preference also positively influenced household monthly expenditure on TLVs. Convenience supply in this study is explained by two items: availability and being sold everywhere. They positively, significantly influenced the average monthly household expenditure on TLVs by 1.24 USD. This finding is similar to the findings of Baker \& Wardle (2003). They reported convenience as an important determinant and typical barrier of consumption and thereby expenditure on vegetables. The intrinsic preference, which is explained by three items (taste, not exotic and easy to prepare), significantly and positively influenced the average household monthly expenditure on TLVs. This confirms the findings that people value TLVs for their taste (Abukutsa-Onyango, 2007). On the other hand, this 
study recognises differences in the preferences among the districts and seasons. For both seasons, households in Binga, Harare and Makonde preferred spider flower, pumpkin leaves and cowpea leaves, respectively. Overall, results show that the most preferred TLVs in descending order are spider flower, pumpkin leaves, cowpea leaves and okra. Mukwereza (2000) reported that spider flower, pumpkin leaves and cowpea leaves are the most preferred TLVs in Zimbabwe, but did not consider locational variations.

\section{Statistically significant interactions}

The interactions between off-season and the variables age of respondent, approximate years of schooling, Mbire and food supply constraints are positive and statistically significant on TLV consumption. On the other hand, Makonde, Mbire and healthiness interactions with offseason showed positive and statistically significant on household expenditure on TLVs. The positive interactions with off-season suggest its influence in both household monthly consumption of and expenditure on TLVs. Such interactions probably can improve household monthly consumption of and expenditure on TLVs, which will be nutritionally beneficial for the persons within those households.

\section{Frequency, market preference and TLV type preference}

Over $50 \%$ of the households in the three districts Makonde, Mbire and Binga collected the TLVs from their gardens/fields, whilst $44 \%$ of the households in Harare bought TLVs from Mbare Musika farmers' market and $26 \%$ from supermarkets. Similar results have been reported by Faber et al. (2010). They reported that most TLVs are not bought but collected from the wild. According to Faber et al. (2010), urban people have limited space to produce TLVs in their gardens 


\subsection{Conclusions and Recommendations}

Households in poor districts generally consume and spend less than in Harare. Consumption of dried TLVs is relatively higher in households in poor dry areas, relate to how the TLVs can be important for improvement of food security in these marginalized areas of Zimbabwe. Also, it is worth noting that product-related characteristics like convenience supply and intrinsic preferences exerted a significant impact on the expenditure on TLVs. For household monthly expenditure on TLVs, all socio-economic variables included in this study gave a positive influence. These findings suggest that TLVs are no longer regarded as food for the poor. TLV preferences depend on location. People consume TLVs because of their healthiness. The study suggests that further nutrition education programs may increase their consumption, otherwise the production of TLVs may not improve and continue to support the people who depend on them. As a general comment, the findings show very low consumption of vegetables per capita in Zimbabwe.

\section{Acknowledgements}

Praxedis Dube would like to thank the Netherlands Organisation for Scientific Research (NWO) for its support through the NFP scholarship. Technical support from her fellow PhD students and Agritex Principal Director Mr. Gondo giving permission to work with his staff are highly appreciated. Lastly, all authors would like to thank the extension officers from Agritex for their substantial support in data gathering. 


\section{References}

Abukutsa-Onyango, M. O. (2007). Seed production and support systems for African Leaf Vegetables in three communities in Western Kenya. African Journal of Food Agriculture and Nutrition Development. 7 (3): 1-16.

Abukutsa-Onyango, M. O. (2010). African Indigenous Vegetables in Kenya: Strategic Repositioning in the Horticultural Sector. Jomo Kenyatta University of Agriculture and Technology. 1-63.

Ambrose-Oji, B. (2009). Urban Food Systems and Traditional Leafy Vegetables: Defining the Spaces and Places for Traditional Leafy Vegetables in Urban and Peri-Urban Agriculture. In: Traditional Leafy Vegetables in urban agriculture. (Shackleton C.M., Pasquini M.W., Drescher A.W., eds.) London, Earthscan. 1 - 33.

Baker, A. H. \& Wardle, J. (2003). Sex differences in fruit and vegetable intake in older adults. Appetite. 40: 269-275.

Banwat, M. E., Lar, L. A., Daber, J., Audos, S. \& Lassa, S. (2012). Knowledge and intake of fruit and vegetables consumption among adult in urban community in north central Nigeria. Nigeria Health Journal. 12 (1): 12-15.

Benson, T. (2017). Agricultural correlates of aggregate nutritional outcomes in Malawi: District-level rank analysis. Outlook on Agriculture. 46 (4): 279-288.

Blisard, N., Stewart, H. \& Jolliffe, D. (2004). Low-Income Households' Expenditures on fruits and vegetables. Economic Research Service/USDA. Agricultural Economic Report Number 833. 1-31.

Croft, M.M., Marshall, M.I. \& Weller, S.C. ( $\left.\begin{array}{lllll}2 & 0 & 1 & 4\end{array}\right)$. Consumers' preference for quality in three African indigenous vegetables in Western Kenya. Journal of Agricultural Economics and Development. 3 (5): 67-77.

Dunn, R. A., Dean, W. R., Johnson, C. M., Leidner, A. \& Sharkey, J. R. (2012). The Effect of Distance and Cost on Fruit and Vegetable Consumption in Rural Texas. Journal of Agricultural and Applied Economics. 44 (4):491-500.

Food \& Agriculture Organization of the United Nations Rome. (2015). Promotion of Fruit and Vegetables for Health Report of the Pacific Regional Workshop.

FAO, WFP \& IFAD. (2012). The State of Food Insecurity in the World 2012. Economic growth is necessary but not sufficient to accelerate reduction of hunger and malnutrition. Rome, FAO. 
Friis, H. (2006). Micronutrient interventions and HIV infection: a review of current evidence. A European Journal of Tropical Medicine and International Health. 11 (12): 18491857.

Gido, E. O., Ayuya, O. I., Owuor, G. \& Bokelmann, W. (2017). Consumer acceptance of Leafy African Indigenous Vegetables: Comparison between rural and urban dwellers. International Journal of Vegetable Science. 23 (4): 346-361.

Hassan, S, Hussain, A., Khan, M. A. \& Mahmood, I. (2012). Rural-urban retail prices and marketing margins of Fresh fruits and vegetables in Pakistan. Pakistan Journal Agricultural Research. 25 (3): 206-217.

Irungu, C., Mburu, J., Maundu, P., Grum, M., \& Hoeschle-Zeledon, I. (2008). The Effect of Market Development On-farm Conservation of Diversity of African Leafy Vegetables around Nairobi. Paper prepared for presentation at the 12 th EAAE Congress 'People, Food and Environments: Global Trends and European Strategies', Gent (Belgium), 2629 August 2008: 1-12.

Jansen van Rensburg, W. S., Venter, S. L. Netshiluvhi. T. R., Heever, E. van den, Vorster, H. J. \& d Ronde, J. A. de (2004). Role of Indigenous leafy vegetables in combating hunger and malnutrition. South African Journal of Botany. 70 (1): 52-29.

Kimiywe, J., Waudo, J., Mbithe, D., Maundu, P. (2007). Utilization and medicinal value of indigenous leafy vegetables consumed in urban and peri-urban Nairobi. African Journal of Food Agriculture and Nutrition Development. 7 (4):1-15.

Mosha, T. C., Pace, R. D., Adeyeye, S., Laswai, H. S. \& Mtebe, K. (1997). Effect of traditional processing practices on the content of total carotenoid, beta-carotene, alphacarotene and vitamin A activity of selected Tanzanian vegetables. Plant Food Human Nutrition Journal. 50 (3): 189-201.

Mukwereza, L. ( $\left.\begin{array}{llll}2 & 0 & 0 & 2\end{array}\right)$. Report on the Study on Processed Indigenous Vegetables (IV's): Mutare city and its Peri-urban areas. A study undertaken as part of activities for the CPHPfunded IV's project. 1-16.

Odhav, B., Beekrum, S., Akula, U. \& Baijnath, H. (2007). Preliminary assessment of nutritional value of traditional leafy vegetables in KwaZulu-Natal, South Africa Short Communication. Food Composition and Analysis. 20: 430-435.

Oral, C. J. \& Love, J. M. (1983). Determinants of household expenditure on fresh vegetables. Southern Journal of Agricultural Economics. 127-132. 
Price, D. W., Price, D. Z. \& West, D. A. (1980). Traditional and nontraditional determinants of household expenditures on selected fruits and vegetables. Western Journal of Agricultural Economics. 21-35.

Ruel, M. T., Minot, N. \& Smith, L. (2005). Patterns and determinants of fruit and vegetable consumption in sub-Saharan African: a multicountry comparison. International Food Policy Research Institute Washington DC, USA, 1-45.

Schippers, R. R. (2000). Traditional Leafy Vegetables. An overview of the cultivated species. Chatham, UK: Natural Resources Institute/ACP-EU Technical Centre of Agricultural and Rural Cooperation Book: 1-210.

Smallwood, D. M. \& Blaylock, J. R. (1984). Household Expenditures for Fruits, Vegetables, and Potatoes. Technical Bulletin No. 1690. 1.40.

Smith, I. F. \& Eyzaguirre, P. (2007). African leafy vegetables their roles in the World Health Organization's global fruit and vegetables initiative. African Journal of Food Agriculture Nutrition Development. 7 (3): 1-17.

Solomon, M. R. (2009). Consumer behavior: buying, having, and being. 8th ed. Book. Upper Saddle River, N. J: Pearson/Prentice Hall: 1-694.

Twine, W., Moshe, D., Netshiluvhi, T. \& Siphugu, V. (2003). Consumption and direct use values of savanna bio-resources used by rural households in Mametja, a semi-arid area of Limpopo Province, South Africa. South African Journal of Science. 99 (9-10): 467-473.

United Nations, Zimbabwe. (2010). Zimbabwe-Country-Analysis-2010-Report

Vincent, V. \& Thomas, R. G. (1961). An agro-ecological survey of Southern Rhodesia: Part I agro-ecological survey. Salisbury, GovernPrinters.

Vorster, I.H.J., van Rensburg, W.J., Van Zij, J. \& Venter, S.L. (2007). The importance of traditional leafy vegetables in South Africa. African Journal of Food, Agriculture, Nutrition and Development. 7 (4):1-13.

Weinberger, K. \& Msuya, J. (2004). Indigenous Vegetables in Tanzania-Significance and Prospects. Shanhua, Taiwan: AVRDC-the World Vegetable Center, Technical Bulletin No. 31, AVRDC Publication 04-600. 1-70.

Weinberger, K., \& Pichop, G.N. (2009). Marketing of African Indigenous Vegetables along Urban and Peri-Urban Supply Chains in Sub-Saharan Africa. In C.M. Shackleton, Pasquini, M.W. \& Drescher, A.W. (Eds.). African Indigenous Vegetables in Urban Agriculture. London. Earthscan: 225-244. 
World Vegetable Center. (2006). Empowering Small Scale and Women Farmers through Sustainable Production, Seed Supply and Marketing of African Indigenous Vegetables in Eastern Africa. Bangkok: AVRDC - The World Vegetable Center and Family Concern. Yang R.Y. \& Keding G.B. (2009). Nutritional contributions of important Traditional Leafy Vegetables. In: Traditional Leafy Vegetables in urban agriculture. (Shackleton C.M., Pasquini M.W., Drescher A.W., eds.) African Indigenous Vegetables in Urban Agriculture. London. Earthscan: 105-143.

Yen, S. T., Andrew, K. G., Rodolfo, M. \& Nayga Jr. (2011). Determinants of fruit and vegetable consumption in Malaysia: an ordinal system approach. AJARE. 55: 239-256.

Zimbabwe National Statistics Agency (ZIMSTAT) and ICF International. (2012). Zimbabwe Demographic and Health Survey 2010-11. Calverton, Maryland: ZIMSTAT and ICF International Inc. 1-470. 


\section{Annexes}

\section{Annex 1}

Attitude is defined as an evaluation of the food items by consumers whether to purchase it or not, can affect the purchasing, which ultimately influences consumption and expenditure. In our study, the term attitude is used to capture factors that supports or constrains the purchasing of TLVs by respondents e.g. 'My household usually purchases TLVs because they are regarded as healthy food'. Likert scale 1 to 5 denoting in the same order; Completely disagree, Somewhat disagree, Indifferent, Somewhat agree and Completely agree was used to capture respondents' attitude when purchasing TLVs and were comprised of 16 items. Factor analysis of the 16 items, that contribute the variable attitude were resolved to six factors with Eigenvalues greater than 0.4 and are presented in Table Annex 1. Healthiness is defined by five items which all relate to the importance of TLVs in improving the people's health.

\section{Table Annex 1 Factor analysis of the 16 items}

$\begin{array}{ll}\text { Factor Name (Variable) } & \text { Items } \\ \text { Healthiness } & \text { Medicinal properties } \\ & \text { Beneficial properties } \\ & \text { Nutritious } \\ & \text { High quality food } \\ \text { Food supply constraints } & \text { Improve family health } \\ & \text { Only vegetables growing in my area } \\ \text { Convenience of TLV supply } & \text { Only vegetables on the market } \\ & \text { Sold everywhere } \\ \text { Readily available } \\ \text { Intrinsic preference } & \text { Like their taste } \\ & \text { Not exotic } \\ \text { Easy to prepare } & \text { Traditional } \\ \text { Source: Authors. } & \text { Reminds me of rural areas } \\ \text { Cocal loyalty } & \text { Locally produced } \\ & \end{array}$

Influence of socio-demographic and socio-economic characteristics, location and productrelated characteristics are presented in Table Annex 2. Results are reported at $p$-value<0.05. 
Table Annex 2 Coefficient of the mean household consumption and expenditure using OLS estimates

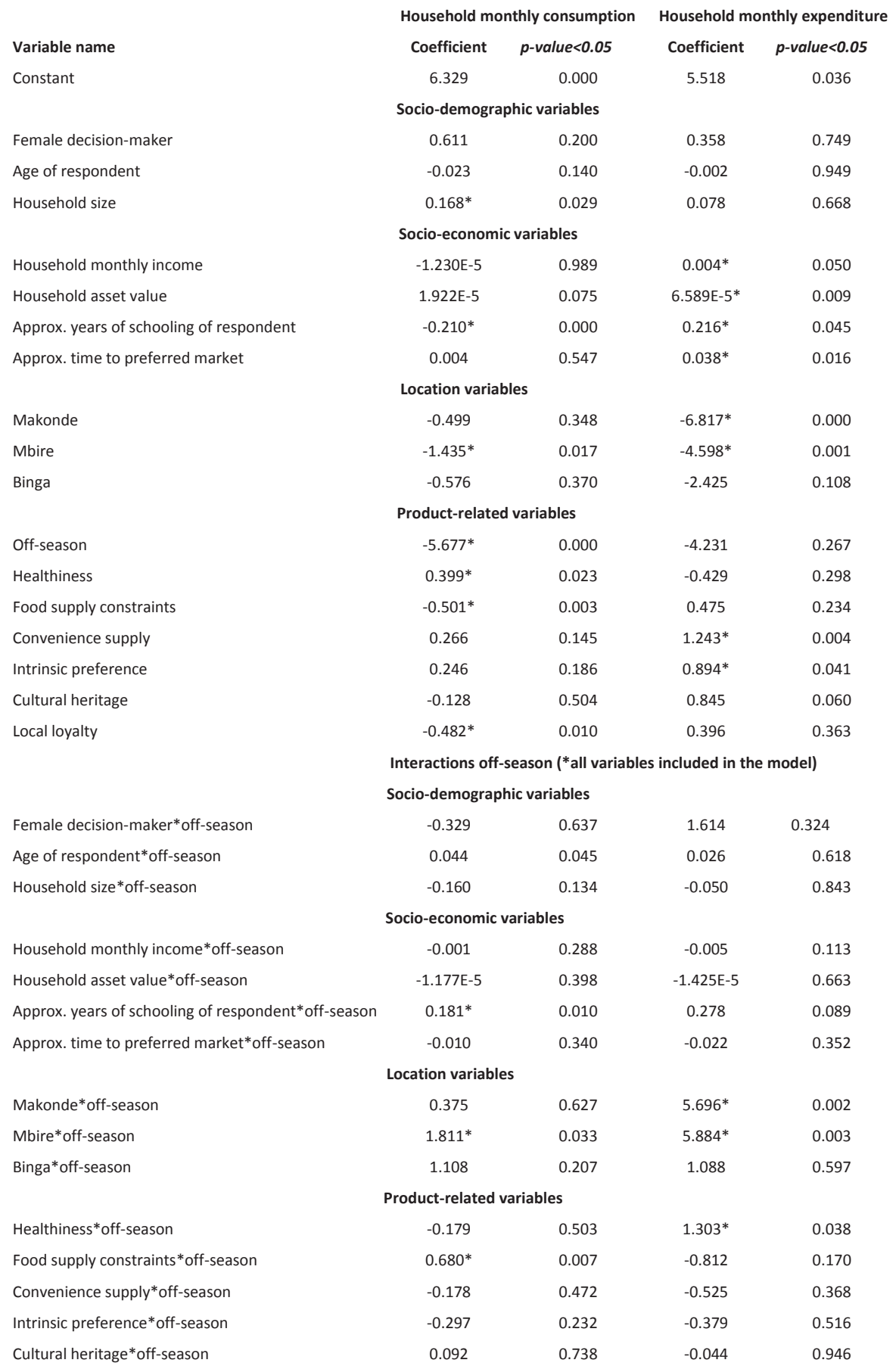


a. Note: * denotes significant at $5 \%$.

b. Dependent variable: Consumption/hh/month/kilograms, age/years, household size/number of persons, household monthly income/USD, household asset value/USD and approximate years schooling/years.

c. Dependent variable: Expenditure/hh/month/USD, age/years, household size/number of persons, household monthly income/USD, household asset value/USD and approximate years schooling/years.

Source: Authors.

Table Annex 3 shows the socio-demographic data of the districts surveyed. Harare is by far the most densely populated while population density is much lower in the other three districts. Since Harare is the capital city of Zimbabwe, people tend to migrate in search of employment. Outside Harare the lowest incidence of household poverty is found in Makonde. The geographical pattern of poverty and therefore food insecurity is partly explained by the degree of rurality, proximity to major urban cities and influence of rainfall patterns. Table Annex 3 suggests that poverty is far worse in sparsely populated districts which are mostly rural. Binga has the highest poverty rate, followed by Mbire. The capital has the lowest prevalence of poverty among the four districts of interest. Table Annex 3 also shows the distribution of household sizes by district. Districts with larger household sizes tend to have a higher prevalence of poverty.

\section{Table Annex 3 Socio-economic data of the districts surveyed}

$\begin{array}{lllll}\text { Variable } & \text { Harare } & \text { Makonde } & \text { Mbire } & \text { Binga } \\ \text { Inhabitants } & 1468767 & 148819 & 81908 & 138074 \\ \text { Average household size } & 4.0 & 4.6 & 4.5 & 4.4 \\ \text { Population density } & 2406 / \mathrm{km}^{2} & 25 / \mathrm{km}^{2} & 40 / \mathrm{km}^{2} & 10 / \mathrm{km}^{2} \\ \text { Area } & 960.9 \mathrm{~km}^{2} & 8564.17 \mathrm{~km}^{2} & 4695.87 \mathrm{~km}^{2} & 15000 \mathrm{~km}^{2} \\ \text { Distance to Harare (tarred) } & - & 157 \mathrm{~km} & 283 \mathrm{~km} & 852 \mathrm{~km} \text { (via Bulawayo) } \\ \text { Average monthly income } & 475.25 \text { USD } & 308.41 \text { USD } & 271.56 \text { USD } & 304.01 \text { USD } \\ \text { Poverty rate } & 31.6 \% & 73.5 \% & 81.0 \% & 88.3 \% \\ \text { Major ethnic group } & \text { Zezuru } & \text { Korekore } & \text { Korekore } & \text { Tonga } \\ \text { Annual precipitation (per year) } & 750-1000 \mathrm{~mm} & 750-1000 \mathrm{~mm} & 450-600 \mathrm{~mm} & >450 \mathrm{~mm} \\ \text { HIV/AIDS by province } & 13 \% & 15 \% & 14 \% & 18 \%\end{array}$

Source: Zimbabwe National Statistics Agency (ZIMSTAT) and ICF International (2012); Poverty and Poverty Datum Line Analysis (2011/12) 
Table Annex 4 Reason for usage as a variable for explaining consumption/expenditure

Explanatory variable

Dummy female decision-maker

Age

Household size

Household income

Household asset value

Years of schooling

Time to preferred market

Dummy Off-season

Dummy Makonde

Dummy Mbire

Dummy Binga

Healthiness (Factor based on Likert scale 1 to 5 )

Food supply constraints (Factor based on Likert scale 1 to 5)

Convenience of TLV supply (Factor based on Likert scale 1

to 5)

Intrinsic preference (Factor based on Likert scale 1 to 5)

Cultural heritage (Factor based on Likert scale 1 to 5 )

Local loyalty (Factor based on Likert scale 1 to 5 )
Reason for usage as a variable for explaining

consumption/expenditure

Capture the influence of female decision-makers on household diet Account for the effect of age variations

Account for the effect of household size heterogeneity

Account for the effect of income variation

Capture the influence of wealth accumulation

Account for the effect of more years of schooling

Account for the effect of travelling time to the preferred market

Account for seasonal influences

Account for the effect of geographical location

Capture the influence of location and food insecurity

Capture the influence of location and food insecurity

Capture the influence of healthiness factors

Account for the effect of production constraints

Capture the influence of convenience

Capture the influence product-related factors

Capture the influence of cultural heritage

Capture the influence of product-related factors

Note:

a. Factor is the variable attitude based on Eigenvalues greater than 0.4 .

b. Likert scale 1 to 5 denotes with respect to the numbers in increasing order Completely disagree, Somewhat disagree, Indifferent, Somewhat agree and Completely agree.

Source: Authors.

\section{Annex 5}

Chinhoyi-Makonde is a district city of Makonde. Growth points are settlements meant to provide employment in rural areas and improve the local economy to curb rural-urban migration. The sectors are a combination of resettlement areas which are more like rural communities except for the fact that they own more assets than the people in rural areas and commercial farming areas. In the third stage, purposive sampling was used to come up with six wards for Mbire and Binga, seven places in Makonde and eight places in Harare. The wards for each district (Mbire and Binga) are political demarcations and were selected based on the following criteria: one of the six wards was a growth point; then out of the remaining five, three were perennially food insecure and the remaining two were moderately food secure in comparison within each district. The fourth stage for Makonde, Mbire and Binga involved 
simple random sampling of villages and households from the list obtained at Agritex offices. Three villages were used for each ward across all districts except for Binga and ChinhoyiMakonde. Within those districts, two wards that had only one village and district city, respectively, were used. The fifth stage involved the stratification of the two urban places Harare and Chinhoyi-Makonde into farmers markets, affluent and middle-income supermarkets and major places like bus terminuses. In Harare, respondents were interviewed at supermarkets, farmers market and bus terminuses, whereas in Chinhoyi-Makonde information was obtained by approaching people at supermarkets and one residential area. In the remaining three districts, respondents were interviewed at randomly selected households, supermarkets and growth points. 
5 The potential of traditional leafy vegetables for improving food security in Africa $^{6}$

\footnotetext{
${ }^{6}$ This chapter has been published as: Dube, P., Heijman, W., Ihle, R. \& Ochieng, J. (2017). The potential of traditional leafy vegetables for improving food security in Africa. Book chapter in: Establishing Food Security and
} Alternatives to International Trade in Emerging Economies 220-243. DOI: 10.4018/978-1-5225-2733-6.ch011. 


\begin{abstract}
Feeding the quickly growing population in Africa remains a global challenge. As the demand for food increases, climate change, on the other hand, poses more challenges to agricultural productivity, implying that the provision of sufficient quantities and qualities of food is threatened. Traditional leafy vegetables (TLVs) in Africa are resilient to adverse weather conditions and are naturally rich in nutrients including vitamins A \& C, iron, protein and other micronutrients. The objective of this chapter is to assess the potential of TLVs improving food security in Africa. TLVs represent a robust local source of food, their consumption is crucial in complementing Africa's diet with micronutrients. TLVs have therefore the potential to play a major role in improving food security and facilitating food sovereignty. Research on seeds, developing seed systems, coupled by preparing and processing methods to TLV products like cakes or flour is needed to increase their consumption, particularly among the young, elite and urban dwellers.
\end{abstract}




\subsection{Introduction}

Feeding the quickly growing population in Africa remains a worldwide challenge. Globally, close to a billion people are hunger stricken $(F A O, 2015)$ and approximately one third of them live in Africa (Sasson, 2012). As the demand for food increases, climate change on the other hand poses challenges to domestic food production in Africa, implying that the provision of sufficient quantities of food of the necessary quality is threatened. A lack of sufficient quantities of sufficiently diversified food compromises human health, resulting in an increase in the risk of contracting diseases related to malnutrition, which affect one in every four people (FAO, 2015). Figure 6 illustrates the prevalence of hunger and the distribution of the undernourished population across Africa. The map reveals substantial differences between African regions. Sub-Saharan Africa is the most severely affected by the prevalence of hunger and undernourishment.

Figure 6 Hunger and undernourishment persistence in Africa in 2015

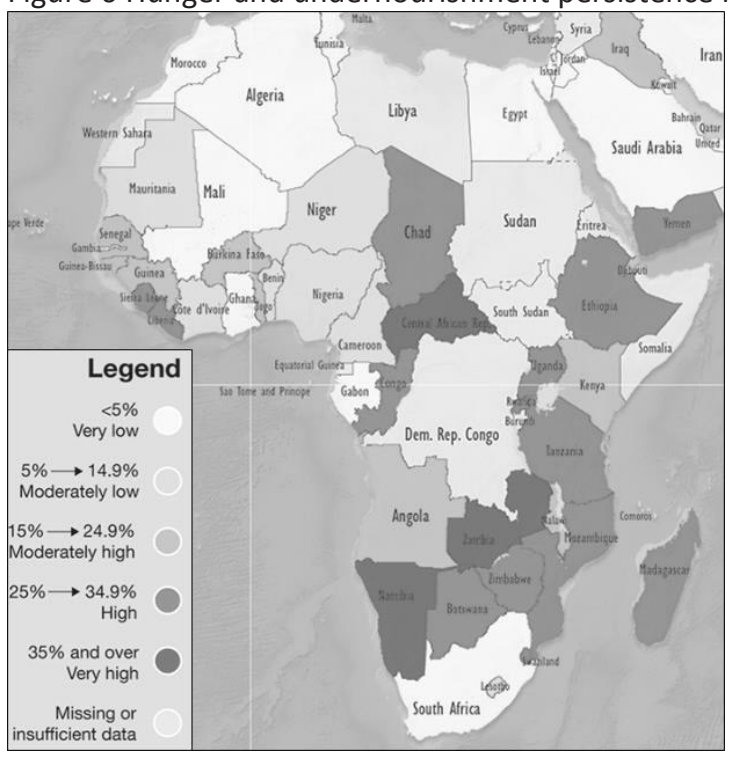

Source: FAO (2015). 
In contrast, Northern Africa accounts for $1 \%$ of the undernourished population, as depicted in Figure 7.

Figure 7 Prevalence percentage of undernourishment in Africa in 2014-2016

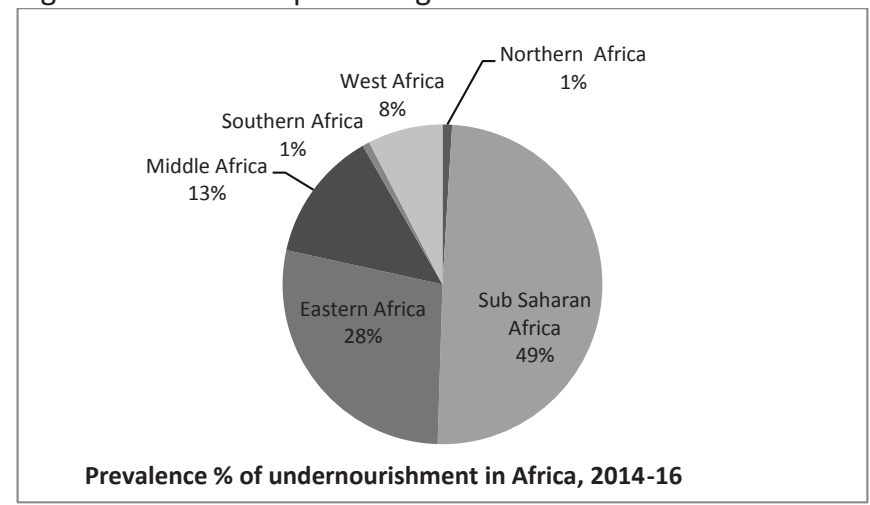

Source: Authors based on FAO (2015).

The differences in undernourishment in Africa are attributed to price volatility, an exploding population, droughts (FAO, 2015), poor governance (Ihle et al., 2009) and political instability (Veninga \& Ihle, in press). For instance, highly volatile food prices affect mainly the poor people in urban and rural areas, since they spend a higher proportion of their limited incomes on their food needs (Luckmann et al., 2014). They tend to consume smaller quantities less frequently as well as cheaper and less nutritious foods that lack vitamins, minerals, and other essential micronutrients, e.g. zinc or iron, both of which are required by the human body for healthy growth. This leads to widespread undernourishment. Vegetables, particularly traditional leafy vegetables (TLVs) might provide dietary elements often missing in other staple foods, e.g. cereals and root foods, which are affordable also for the poor population. They might be used as primary sources of minerals because they are naturally rich in nutrients such as iron and other micronutrients that are important for a human's well-being. They have also the potential to balance diets and make them healthier as they also include vitamins $A$ and $\mathrm{C}$, and proteins. The objective of this chapter is therefore to assess the potential of TLVs for improving food security in Africa. 


\subsection{Background}

The current status of agriculture in Africa

Agriculture contributes significantly to Africa's economy. It is responsible for a relatively large share of the economy in terms of labour force. It accounts for $70 \%$ of jobs, contributes $33 \%$ of the gross domestic product, and generates $40 \%$ of the total export earnings in Africa (Otsuka et al., 2013). Africa faces the challenge of low food productivity due to a number of factors, including poor uptake of modern technologies, and high incidences of poverty culminating in the prevalence of an undernourished population among others. At the United Nations Sustainable Development Summit on September 25, 2015, world leaders adopted the Sustainable Development Goals (SDGs) to end poverty, fight inequality and injustice, and tackle climate change by 2030 (United Nations Development Programme [UNDP], 2016).

The modern technologies have resulted in the replacement of TLVs with the conventional vegetable varieties such as rape, cabbages etc. However, these exotic vegetables usually grow in high-altitude areas with a cool climate and require modernized technologies, like irrigation which is far beyond the reach of many smallholder farmers in Africa. In the humid lowland areas of West Africa, the arid areas of Namibia and Botswana, in the blistering heart of Kalahari, in hot dry regions in other parts of the continent, the exotic vegetables cannot grow well, but TLVs can grow (Schippers, 2000) allowing for the effective use of non-productive areas.

\section{Traditional leafy vegetables in Africa}

Traditional leafy vegetables are plants which originate from the African continent and have a century-long history of cultivation and domestication subject to regional agro-climatic conditions (Ambrose-Oji, 2009). They are often more resilient to adverse weather conditions and withstand better unpredictable weather changes than varieties of staple crops and vegetables bred in industrialized countries, fitting very well to bioregional foods.

Traditional leafy vegetables are naturally important to many people in Africa due to their inherent nutrient content and vast range of phytochemicals (Yang \& Keding, 2009). Generally, 
TLVs are more nutritious than commonly known exotic vegetables. Commonly consumed vegetables across Africa include Amaranth (Amaranthus spp.), Okra (Abelmoschus esculentus), Spider flower (Cleome gynandra), Pumpkin leaves (Cucurbita spp.), Cowpea leaves (Vigna unguiculata), Black jack (Bidens pilosa), Sweet potato leaves (Ipomoea batatas), Nightshade (Solanum spp.), Moringa (Moringa oleifira), African eggplant (Solanum spp.), Baobab leaves (Adonsonia digitata), Jute (Corchorus olitorius), and Cassava leaves (Manihot esculentum).

Table 17 shows laboratory results on the nutritional contents of TLVs found by the World Vegetable Center, TLVs have substantial amounts of micronutrients, vitamins and minerals which are essential for a balanced and healthy diet. These vegetables often have higher amounts of nutrients than vegetables which are traditionally grown on other continents such as tomatoes and cabbage. For example, Moringa provides the highest quantity of micronutrients among the most used TLVs. TLVs are generally rich in vitamins $A, C$ and $E$, zinc, calcium, iron, and antioxidants (Yang \& Keding, 2009). In comparison, TLVs have higher nutrient amounts than other vegetables such as tomatoes and cabbages, Table 17.

Table 17 Micronutrient content of common TLVs

\begin{tabular}{|c|c|c|c|c|c|c|}
\hline & & & Vegetable typ & & & \\
\hline Micronutrient & Range & Tomato & Cabbage & Moringa & Amaranth & $\begin{array}{c}\text { Sweet potato } \\
\text { leaf }\end{array}$ \\
\hline$\beta$-Carotene, mg & $0.0-22$ & 0.40 & 0.00 & 15.28 & 9.23 & 6.82 \\
\hline Vit C, mg & $1.1-353$ & 19 & 22 & 459 & 113 & 81 \\
\hline Vit E, mg & $0.0-71$ & 1.16 & 0.05 & 25.25 & 3.44 & 4.69 \\
\hline Iron, mg & $0.2-26$ & 0.54 & 0.30 & 10.09 & 5.54 & 1.88 \\
\hline Folates, $\mathrm{mg}$ & $2.8-175$ & 5 & ND & 93 & 78 & 39 \\
\hline $\begin{array}{l}\text { Antioxidant } \\
\text { activity, TE }\end{array}$ & $0.6-82,000$ & 323 & 496 & 2,858 & 394 & 870 \\
\hline
\end{tabular}

Some TLVs can be produced or collected from forests or fallow lands. In Eastern and Western Africa, TLVs production is commercial in both urban and peri-urban areas (Weinberger \& Pichop, 2009). Most farmers producing TLVs sell about $50 \%$ of the produce through different market channels, and the remainder is consumed within households. Table 18 shows that the 
total volume of TLVs produced by seven countries was 9,300 tons/year (Weinberger \& Pichop, 2009). The quantity is relatively small given that it is produced by seven countries. The total volume was largest in Eastern Africa, followed by Western Africa. The production of TLVs in these three parts of Africa is driven by generating extra income and home consumption, among others (Weinberger \& Pichop, 2009). Given their nutritional potential, promotion campaigns such as road and cook shows, nutritional awareness and educational programs in hospitals, schools and markets have been used, for example in Tanzania to increase consumption of traditional vegetables by rural and urban consumers (Ochieng et al., 2016).

Table 18 Total TLVs production by African regions in 2016

\begin{tabular}{lc}
\multicolumn{1}{c}{ Region (countries) } & Total volume (tons) \\
Eastern Africa (Kenya, Tanzania, and Uganda) & 6900 \\
Western Africa (Senegal, Benin, and Côte d'Ivoire) & 2400 \\
Southern Africa (South Africa) & 27 \\
Total volume & 9327
\end{tabular}

Source: Authors based on IndigenoVeg survey data Weinberger \& Pichop (2009).

The quantities consumed per day vary across the African regions. For instance, in Southern Africa, $29-41 \mathrm{~kg} /$ year are consumed by South Africans/households depending on the location (rural or urban dwellers, with the latter consuming less) (Yang \& Keding, 2009). A study of the TLVs consumption in seven countries of Africa showed that Figure 8 Tanzania consumed largest quantities of TLVs/capita/day, while Senegal consumed the least, as shown in Figure 8. On average, Eastern African countries consumed larger quantities, indicating the importance of nutrition awareness.

Figure 8 TLVs per capita per day (grams) by country

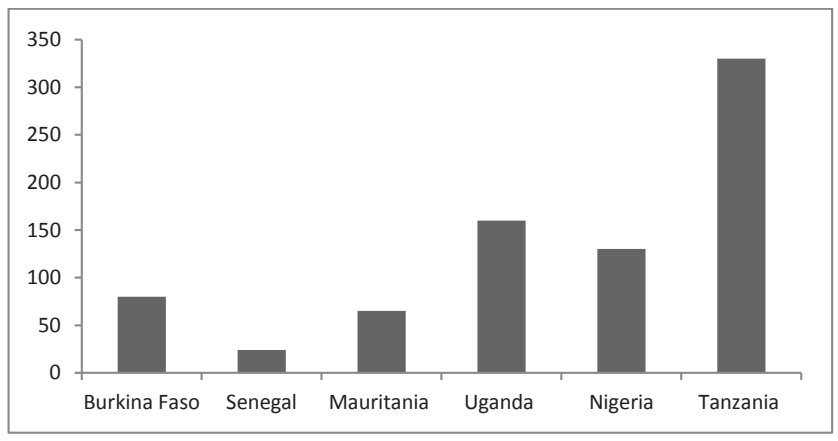

Source: Authors based on IndigenoVeg consortium data Weinberger \& Pichop (2009). 
Over the last two decades in the context of the realization of the limitations of the Green Revolution, the national food programmes and strategies, and the effects of climate change, researchers realized a need for a paradigm shift to look at underutilized plants such as TLVs. These neglected species have begun to attract considerable interest due to their multiple underexploited benefits in terms of nutritional value, food security, income generation, medicinal value, and suitability for low input systems and for marginal environments (Shackleton et al., 2009). The interest in the underutilized species for research and development came during the late 1980s. For example, there was the establishment of the International Centre for Underutilized Crops in 1988, the Plant Resources of Tropical Africa in 2000, the Global Facilitation Unit of Underutilized Species in 2002, Consultative Group on International Agricultural Research in 2005 and the inclusion of indigenous vegetables for biodiversity, healthy diet and marketing opportunities as one of the World Vegetables Centre's strategic programme directions in 2002 (Shackleton et al., 2009). All these strategies aimed at increasing the use of the underutilized crop species such as TLVs to retard biodiversity losses, increase community driven rural development, exploit the nutritional value, food security, income generation and medicinal value and make marginal lands more productive.

Recent milestones in TLVs production and consumption include a significant growth of interest in re-activating traditional food and a reversal of an earlier decline in farming of indigenous crops that this food is based upon. Scientists in Africa and elsewhere have been engaged in research to develop TLVs in order to tap into their health benefits (Cernansky, 2015). These researches have created new opportunities for TLVs in many countries. A study on TLVs production has shown that Kenyan farmers have increased the TLVs production area by $25 \%$ between 2011 and 2013 (Cernansky, 2015). Also, the number of bunches sold in Kenya have been increasing rapidly, estimated at $164 \%$, as depicted in Figure 9 . The TLVs have gained a commercial status in large cities like Nairobi (Irungu et al., 2011). Some TLVs, e.g. African nightshade, have gained $32 \%$ in the total Nairobi vegetable markets share (Irungu et al., 2011) and 11\% in Tanzania. The World Vegetable Center is currently budgeting annually around \$20 million on TLVs research (Cernansky, 2015). 
Figure 9 Supply trends of TLVs in Nairobi markets

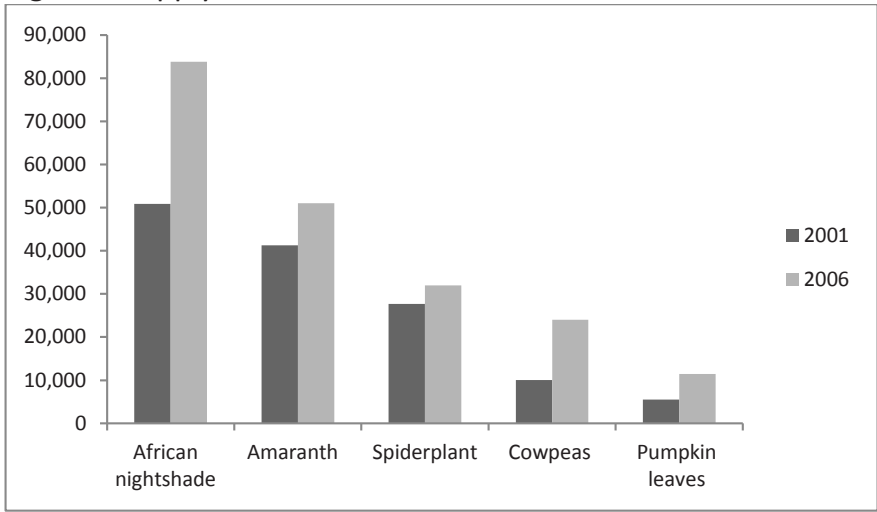

Source: Authors based on Irungu et al. (2011).

\subsection{Main focus of the Chapter}

\section{Current status of traditional leafy vegetables in Africa}

TLVs play significant roles in the livelihoods of people along the supply chain in Africa. They contribute approximately 13\%, 35\%, and $30 \%$ of the smallholders' income in Tanzania, Malawi, and Mozambique, respectively (Weinberger \& Msuya, 2004; Chagomoka et al., 2014), $29 \%$ to $76 \%$ in Uganda, Côte d'Ivoire, Kenya, Tanzania, Senegal, South Africa, and Benin (Weinberger \& Pichop, 2009). Figure 10 depicts the share TLVs farmer receive of the consumer selling price. The data shows substantial differences between African countries. Higher shares are realized in South Africa and Benin. The differences in shares can be partially explained by prices. For example, a farmer in South Africa realizes better shares due to the price difference per kilogram of cowpea leaves selling at 3.20 USD for South Africa compared to 0.22 USD for Tanzania (Weinberger \& Pichop, 2009). 
Figure 10 TLVs farmers' percentage share in consumer selling price

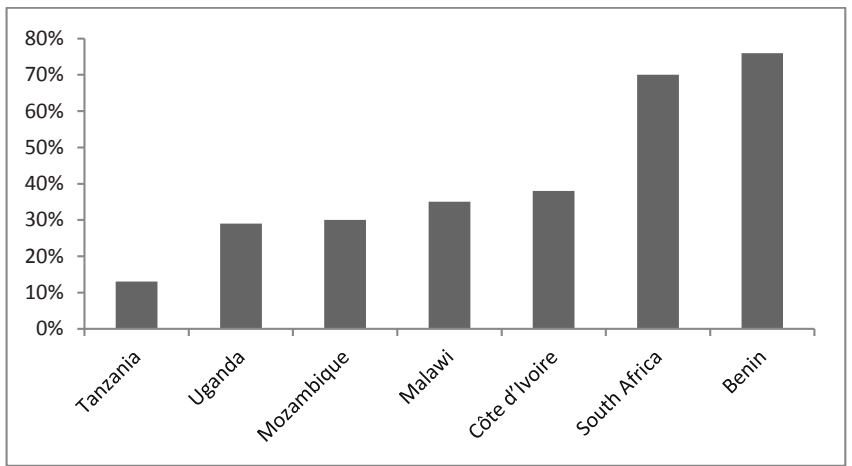

Source: Authors based on Weinberger \& Msuya (2004); Weinberger \& Pichop (2009); Chagomoka et al. (2014).

A market study conducted in seven countries within Africa in 2009 shows the total volume and total turnover for the three most important TLVs traded at retail levels, as depicted in Figure 11. This study established that TLVs are relatively high value crops generating a total turnover of 5.5 million USD (Weinberger \& Pichop, 2009). Assuming the same turnover being produced by 53 African countries, calculated, would yield approximately 50 million USD generated by TLV supply chain markets.

Kenya shows the highest volume traded for the three most important TLVs, almost thrice the volume traded in Senegal yet with the total turnover lower than that of Senegal. South Africa exhibits the least total volume traded, but its total turnover is higher than that of Côte $d^{\prime}$ Ivoire. For the rest of the countries included in this data, only Senegal and South Africa have a total turnover higher than the total volume traded. These differences can be partially explained by the difference in selling prices (USD/kg) at retailers between these countries. For example, cowpea leaves selling per kilogram at 3.64 USD and 3.20 USD in Senegal and South Africa, respectively, compared to 0.30 USD, 0.45 USD, 0.30 USD, 0.22 USD for Benin, Kenya, Uganda, and Tanzania, respectively (Weinberger \& Pichop, 2009). 
Figure 11 Total volume and total turnover for TLVs traded at retail/ country

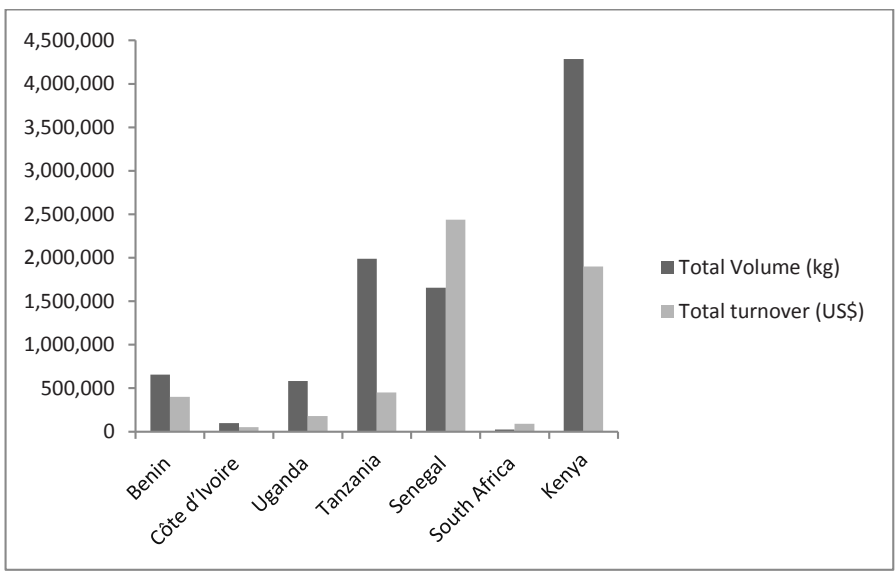

Source: Authors based on IndigenoVeg survey data Weinberger \& Pichop (2009).

For TLVs, the trader and retailer share contribution at consumer selling point is the lowest in Benin at $24 \%$ and in South Africa at $30 \%$. It is the highest in Uganda (7 $1 \%$ ), Côte d'Ivoire (6 $2 \%$ ), and Tanzania (around 55\%) (Weinberger \& Pichop, 2009). These differences can be partly explained by the share that is allocated to the farmers, e.g. the share received by farmers is relatively higher in Benin and South Africa, as shown in Figure 11.

\section{Supply chains of traditional leafy vegetables}

The authors assessed the role of TLVs in small-scale farmers' diets and incomes, the survey was conducted in five provinces of Zimbabwe (Mashonaland East, West and Central, Harare, Manicaland). The respondents were chosen using the snow ball technique, starting with the seed production and supply segment upstream to traders, gathering data from five key informants within each segment. The authors developed question guides to help consistent data collection and interview technique at each segment. The information collected included crop management, marketing of the produce and information, among others. The content of this chapter is partly based on the results obtained from the survey.

The TLV supply chain is very short, due to high perishability. During the harvesting process, farmers normally place TLVs under the shade and at times sprinkle water on the vegetables. In Zimbabwe, farmers or intermediates pack TLVs in jute bags, and then transport the product 
to the markets. Some bags are packed in big lorries or placed on top of the commuter buses and tied by a rope to avoid falling. Transportation is done during the evening when temperatures are low. Unfortunately, there is no appropriate storage during transportation and at the market places, which can result in reduced shelf life of vegetables. To avoid losses through spoilages, the producers and traders are forced to bring or buy the TLVs in small enough quantities such that they can be sold within a day or two.

The producers minimize the transactional costs and losses due to high perishability by taking the TLVs directly to the markets. In such cases, it may be worthwhile for the producers to have market information before taking the TLVs to the markets. Alternatively, the producers sell their TLVs to intermediates moving around their farm gates and sourcing the vegetables. In that way, the producers would reduce transport costs and other costs that can be incurred, i.e. high market fees. For example, in Harare, the farmer is charged 10 USD per day, which means that if the farmer has unsold TLVs, the farmer either has to pay another 10 USD for the following day, is forced to dump the remaining product or give it away for free. Figure 12 depicts an example of the movement of TLV seed and vegetables from the areas of production to markets. The TLV seed are primarily produced, sold and exchanged locally, with many people deriving their incomes and livelihoods through various activities carried along the supply chain, thus having the potential for facilitating the attainment of the current imperative to foster food and seed sovereignty and strengthen several of their components. Since their production is local, farmers have control over seeds and the resulting vegetables can then be quickly supplied to consumers, supporting domestic marketing structures in developing countries. This chain supports the flow of purchasing power from more affluent urban regions to rural areas, as depicted in Figure 12. 
Figure 12 TLVs seed production regions and markets in Zimbabwe

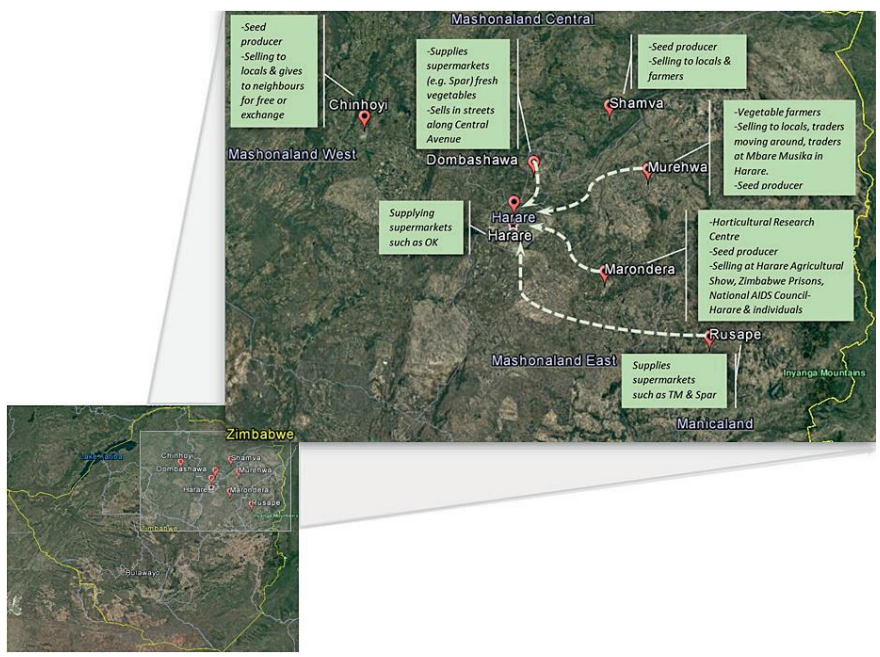

Source: Authors.

The highly perishable nature of TLVs make them unsuitable for long-distance transport. This statement is true across Africa. These vegetables are sold to neighbouring urban areas and locals and in small quantities in processed form are exported to neighbouring countries. For example for Zimbabwe, Botswana and South Africa are importers. Most of the production areas are near urban areas, while there exist others that are located more than $100 \mathrm{~km}$ away from the urban areas. In Zimbabwe, locations where the authors collected data are around $100 \mathrm{~km}$ away from the major markets, translating to an average of three to four hours to reach the market using public transport. In Kenya, the TLV production areas are near Nairobi, whereas others are more than $100 \mathrm{~km}$ away and other retailers had to walk $15 \mathrm{~km}$ daily for fresh TLVs (Nekesa \& Meso, 1997). In Zambia, Lusaka supermarkets source their TLVs from local farmers in the peri-urban areas. In Uganda, the average distance travelled and time used to travel in order to sell the TLVs is not large. It rarely takes more than an hour and the travelling distance is around $14 \mathrm{~km}$ (Weinberger \& Pichop, 2009). TLVs are very perishable and there is a need to produce them near or at the point of use.

In the community, the TLVs are sold at various market outlets, including at farm gates, on the side of the road and door-to-door. In this scenario, the traders are producers as well, as they sell their TLVs directly to consumers and to some intermediaries. Some of the intermediaries 
visit various farm gates, purchase the TLVs, transport them to the markets and eventually sell them to the consumers. It is mainly men who do the purchasing. In case the TLVs are not bought, in the case of Zimbabwe, the producers would process them and sell them later, some would be consumed in their households.

Women play significant roles in the TLV supply chain as producers, processors, and marketers (Weinberger \& Pichop, 2009). In Uganda, men mainly act as intermediaries, as they move around to buy TLVs from producers (mostly women) and transport them to markets. In the markets, women buy them from the intermediaries and then sell these vegetables to the public. Women are, therefore, economically empowered as they are able to pay fees for their children and provide food their households.

Vegetable varieties, seed production and farming characteristics

Many TLV types are produced within homesteads, sold in local markets, exchanged as seeds or sold in the local markets, fostering localized developments. Despite their high potential in offering better opportunities for gender oriented developments, transforming the rural livelihoods through localized markets and addressing food insecurity problems, there are many challenges persisting along the supply chain. The availability of seed and undeveloped seed systems, depriving farmers' access to high quality seed of preferred varieties. More so, with the supplies of vegetables now getting into big cities (increased demand for vegetables), this translates to increased demand for seed as well.

In Eastern Africa, mostly, TLV seed is produced by farmers. Seed stockists buy this seed and then sell it to many emerging TLV farmers. These seed producers are registered with renowned companies and are able to produce certified seed, giving the seed a commercial market value. They are capable of producing high quality seed with the supervision of big seed companies. More so, the TLV seed markets are organized well, enabling seed producers to realize profits and incentivize. The farmers are assured of the ready markets and they know well before planting the types of TLVs preferred by their targeted markets. Unlike in Eastern Africa, Southern Africa, Western Africa and Central Africa, the production of TLV seed has not been formalized. There, no commercial seed company has shown interest in venturing into the seed production and marketing sector. One example where this has happened is 
Cameroon. Various international organizations funded the training and production of TLV seed, aiming at improving seed availability to farmers through the involvement of local seed companies in Eastern Africa and a few in Southern Africa. The top five selected for increased production, consumption and marketing include cowpea, amaranth, nightshade, spider flower, and African eggplant.

In Zimbabwe, TLV seed is produced by a government institute under the Department of Research and Specialist Services (Horticultural Research Centre) and by farmers dotted around the country. The most common TLV seed produced include spider flower, cowpea, amaranth, black jack, okra, tsunga, and pumpkin, with the most traded being spider flower and pumpkin seed. Similarly, findings by Chagomoka et al. (2014) revealed that TLV seed produced in Malawi included Ethiopian mustard, okra, pumpkin, and African eggplant. In Mozambique, the same authors found that most TLV farmers use returned seed, with some of the seed imported from Tanzania. The seed is usually packed in a plastic bag or plastic coated paper. In Zimbabwe, three main sections of TLV seed production were found, notably, the low section (subsistence farmer without knowledge of seed production), middle section (commercialized farmer with relative basic knowledge of seed production) or high section (commercialized seed producing company i.e. Horticultural Research Centre). The seed from the high section was more expensive than other seed sections, costing 50 USD per kilogram. The seed is marketed to different channels: to neighbours, at agricultural shows, and to various organizations promoting the use of TLVs for nutrition and health benefits.

The farmers generally measure out the seed in table spoonfuls or pet coke cola lids. A table spoonful is estimated to weigh approximately 15 grams and a pet coke cola lead is approximately 2 grams, marketed at 2 USD and 0.50 USD respectively or 50 USD per kilogram in Zimbabwe markets and 1.80 USD per 25g of amaranth seed in Kenya markets (Mumbi et al., 2006). When these seed weights and prices were calculated and estimated per hectare of spider flower and amaranth for one season, the gross income presented in Table 19 are derived. In comparison, spider flower seed's gross margin is higher than that of amaranth seed, valued at 6500 USD/hectare. The gross margin for farmers in the production of TLVs vegetables varies due to the TLV type. The least gross margin is obtained from cowpeas with 2426 USD. Nevertheless, in Zimbabwe, the TLV seed is rarely packaged, labelled and traded 
directly to the public without hiring the chain of people or companies to market the seed. Seed produced is sold locally to neighbours, friends and relatives or nearby urban areas. The transactions are mostly based on the cash market.

\begin{tabular}{clllll}
\multirow{2}{*}{$\begin{array}{c}\text { Table } \\
\text { Category }\end{array}$} & TLV grown & Country & Production cost & Gross income & Gross margins \\
Seed (per ha and season) & Spider flower & Zimbabwe & 1000 & 7500 & 6500 \\
Seed (per ha and season) & Amaranth & Kenya & 185 & 3145 & 2960 \\
Vegetables (0.1 per ha/year) & Amaranth & Kenya & 1142 & 5714 & 4571 \\
Vegetables (0.1 per ha/year) & Cowpea & Kenya & 1306 & 3733 & 2426 \\
Vegetables (0.1 per ha/year) & Nightshade & Kenya & 1633 & 4666 & 3033
\end{tabular}

Source: Authors and Mumbi et al. (2006).

\section{Production of traditional leafy vegetables}

Similar to countries in Eastern and Western Africa, the production of TLVs can be commercialized in Zimbabwe, in particular areas close to major cities. For example, a Zimbabwean farmer who produces and sells the TLVs throughout the year as opposed to seasonally gets better prices when the market supplies are low, while also taking advantage of the fast moving of the TLVs when incoming supplies dwindle. The spider flower fetches the highest price and sells fastest. TLVs winter production is mainly carried along major rivers in some parts of Zimbabwe (Mpala et al., 2013), concentrated in irrigation schemes during the dry seasons and in rural areas during the summer season. Most of the TLVs produced are consumed domestically, either by households or through local markets in nearby urban areas. Similarly, the production and marketing of TLVs has been increasing and becoming increasingly commercialized in Kenya, particularly within Nairobi and the peri-urban areas (Irungu et al., 2011). Women participation in the TLVs supply chain is constantly high in Kenya, Benin and South Africa (Table 20). Female participation in TLV production tends to be lowest to other levels of the supply chain. Table 20 shows that it is especially low in Côte d'Ivoire and Senegal.

However, production of TLVs is hindered by unavailability of certified or high quality seed coupled with the underdeveloped seed systems. The underdeveloped seed systems limit the seed movement, subsequently limits the farmers' variety of choices. 
Table 20 Share of women along the TLVs supply chain across countries (\%)

\begin{tabular}{llllllll} 
Category & \multicolumn{7}{c}{ Country } \\
& Benin & Côte d'Ivoire & Senegal & Kenya & Tanzania & Uganda & South Africa \\
Farmers & 40 & 16 & 14 & 59 & 34 & 37 & 65 \\
Intermediaries & 78 & 100 & 26 & 95 & 75 & 69 & unknown \\
Retailers & 100 & 100 & 58 & 86 & 58 & 68 & 86
\end{tabular}

Source: Weinberger \& Pichop (2009).

\section{Processing of traditional leafy vegetables}

Processing and packaging is a way of minimizing spoilage of TLVs due to their high perishability. During the periods when the TLVs are abundant in the markets and in gardens, TLVs are partly processed for future use in the dry season or for distant marketing (Mpala et al., 2013). In Zimbabwe, 97\% of the TLV farmers process parts of the vegetables harvested (Mpala et al., 20103 ), in Uganda 1.2 \%, in Côte d'Ivoire $9.3 \%$, in Kenya 2.1 \%, in Senegal 0.8 \%, and in South Africa 10.3\% (Weinberger \& Pichop, 2009). Similarly, Chagomoka et al. (2014) notes that TLVs are processed prior to selling as a way of minimizing quantity and quality losses. Only $12 \%$ of retailers in Malawi and $6 \%$ of retailers in Mozambique process the TLVs prior to selling. About $50 \%$ of the households in South Africa preserve vegetables for both selling and own consumption.

There are various methods of processing, including; sun-drying without any pre-treatment, blanching and sun-drying and blanching then freezing (Bioversity International, n.d.). Sundrying without any pre-treatment method is the most preferred among the commonly preserving methods (Mpala et al., 2013). In Zimbabwe, cowpea tender leaves are harvested, boiled, and dried in direct sunlight. They are twisted by rolling them between the pumps. The processing varies across the nations, regions and cultures. For example, in Malawi cowpea leaves are processed by sun-drying and/or blanching (Chagomoka et al., 2014), but not twisting. The pumpkin leaves are sold fresh across Africa. Except in some countries in Southern African, including Zimbabwe, they are traded in their dried form. They are dried without cooking and normally their flowers are added.

In Zimbabwe, drying is generally done in a semi-closed cabinet that protects the processed vegetables from fine dust particles that may settle on it in order to avoid making them gritty. Quality attributes such as twisted and dried for cowpea, absence of pathogens, i.e. dried 
without visible molds, and neatly packaged with the labels showing shelf life, net weight and processors' details are mandatory. The concerns arise especially if the quality attributes are not met. This can result in negative perceptions among some urban dwellers and educated elite in response to their fear for their health. The worries related to the health risks were highlighted in the World Vegetable Center (2006) report on determination knowledge and use of TLVs by the elite urban dwellers. The average to middle-income earners consumed little in fear of risking their health.

\section{Marketing channels of traditional leafy vegetables}

Trading TLVs in Kenya dates back to the 1960s (Irungu et al., 2011). The trade of TLVs is highly specialized, as is the consumption. Nairobi has ten large markets, which sell TLVs in larger amounts. These markets are located in residential places and serve the surrounding consumers. Supermarkets and green grocery stores serve the affluent minority who have limited time to visit some of the city council markets, such as Harare and Nairobi City Council markets. In addition, there are several evening traders along the streets who target the people coming from work.

Similarities in trading of TLVs can be found across African regions. In Zimbabwe, the authors noted that three quarters of traders interviewed purchased the TLVs from farmers moving around the market looking to sell to traders they know. While supermarkets in both Kenya and Zimbabwe buy directly from individuals, groups or processors. In comparison, Zimbabwean supermarkets mainly sell dried TLVs with the exception of pumpkin, spider plant, and amaranth leaves, while Kenyan supermarkets sell fresh TLVs.

The traders purchase a bundle of TLVs or two cups of dried TLVs for 0.50 USD. A standard cup consists of around $60 \mathrm{~g}$. The traders split the bundle into two and each is sold to consumers at 0.50 USD. In Zimbabwe, a sachet weighing approximately $80 \mathrm{~g}$ sells for 2 USD in the green grocery, while a range of 2 USD to 2.35 USD, depending on the TLVs type, for $60 \mathrm{~g}$ is sold in supermarkets. Similarly, in Kenya the wholesaler buys a bundle of TLVs for KES2 (0.04 USD), splits the bundle into two and sells each for KES2 to the retailer. In turn, the retailer further splits the bundle before selling it to the consumer (Nekesa \& Meso, 1997). In Zambia, a bundle of TLVs weighing $500 \mathrm{~g}$ is sold between 500 and 800 Zambian Kwacha (0.25 USD -0.30 USD). 
The profit made on TLV sales is over 100\% in Zimbabwe (Mpala et al., 2013), 33\% in Tanzania, and over $75 \%$ in Kenya (Nekesa \& Meso, 1997).

The major TLV dominate marketing channels across Africa, including city council markets, supermarkets, green-groceries, open markets and roadside, as reported by Irungu et al. (2011) and Schippers (2000). Table 21 shows some of the most popular TLVs consumed and found most tradeable in African regions. The types of TLVs popularly consumed and found most tradeable vary substantially by region, except for amaranth. Amaranth is sold fresh in all cities (Maundu et al., 2009). Contrary, spider flower is sold in the fresh or dried form in the Southern Africa region. These variations can be explained by different attributes, including cultural backgrounds, geographical location, and diversity. In comparison, in Western Africa baobab leaves are some of the most popular TLVs consumed, whereas black jack is the most commonly consumed TLVs in the Southern African region. Northern Africa, due its climate conditions, a few types of TLVs exist in the region.

Table 21 Some of the most popular TLVs consumed and traded in Africa

\begin{tabular}{lll}
\multicolumn{1}{c}{$\begin{array}{c}\text { Region } \\
\text { Southern }\end{array}$} & Amaranth, Okra, Spider flower, Pumpkin leaves, & \multicolumn{1}{c}{ Tradeable TLVs } \\
Africa & Cowpea leaves, Black jack, Sweet potato leaves & $\begin{array}{l}\text { Spider flower, amaranth, black jack, cowpeas and pumpkin } \\
\text { leaves, okra, African eggplant, amaranth, jute mallow, Ethiopian } \\
\end{array}$ \\
Eastern & Amaranth, Cowpea vegetables, Pumpkin vegetables, & African nightshade, amaranth, Spider flower cowpea leaves, \\
Africa & Corchorus, Nightshade, Spider flower, Sweet potato & crotalaria, okra, Ethiopian kales, Ethiopian mustard, sweet \\
& leaves, Moringa & potato leaves, cassava leaves \\
Western & Amaranth, African eggplant, Corchorus, Okra, Sweet & Sweet potato leaves, African nightshade, cassava leaves, \\
Africa & potato leaves, Cowpea leaves, Baobab leaves, Spider & pumpkin leaves, jute, Amaranth \\
& flower, Moringa, & \\
Central & Amaranth, Pumpkin leaves, Sweet potato leaves, & Moringa, jute, hibiscus, okra, amaranth, cowpea leaves \\
Africa & Okra, Jute, Spider flower, Cassava leaves &
\end{tabular}

Source: Authors; Nekesa \& Meso (1997); Maundu et al. (2009); Irungu et al. (2011); Chagomoka et al. (2014).

Challenges in realizing the economic potential of traditional leafy vegetables

TLVs have for decades not been recognized as potential contributor to food and nutrition security by policy makers (Schippers, 2000). Only recently, TLVs have become important food crops and have attracted international spotlight because of their potential for human nutrition by providing essential micronutrients, photochemicals, and antioxidants for achieving a balanced diet. The realization of their potential is subject to a number of 
challenges persisting along the TLV supply chain. Major challenges include the unavailability of quality-certified seed to enhance productivity, undeveloped seed systems depriving farmers' choices of varieties, limited preservation methods given their high perishability, resulting in poor quality, processed products, and negative perceptions among some of the young generation, educated elite, and urban dwellers.

Results from a study conducted by Weinberger \& Pichop (2009) in three regions of Africa (Eastern, Southern, and Western), show that the median and inter-quartile range for urban areas dedicated to vegetable production is larger in Nairobi and Kisumu, for peri-urban areas of Cotonou, Durban, Lokossa and Shoshangwe, reflecting increased demand of TLVs in the urban areas in Kenya (Figure 13 and Figure 14).

Figure 13 Cultivated areas in urban and peri urban for six cities

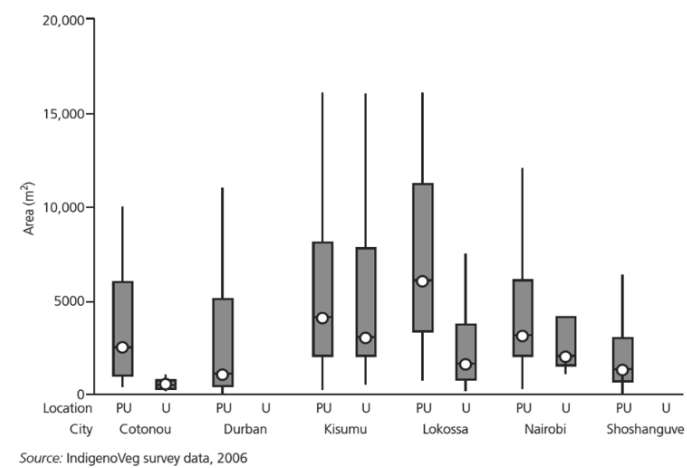

Notes: Median values and inter-quartile ranges are displayed

Source: Authors based on IndigenoVeg survey data Pasquini et al. (2009). 
Figure 14 Vegetable production in urban and peri-urban areas of six major African cities

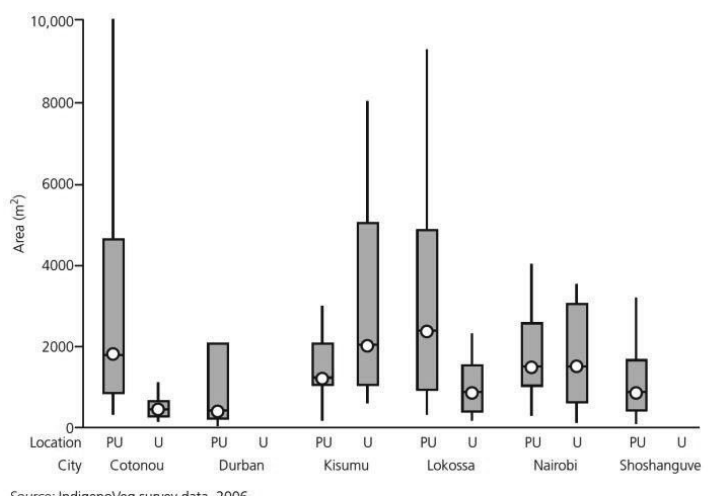

Source: IndigenoVeg survey data, 200

Notes: Median values and inter-quartile ranges are displayed

Source: Authors based on IndigenoVeg survey data Pasquini et al. (2009).

If the quality of TLV seed would be improved and become more homogenous, farmers might additionally benefit by having less expenditures for the seed and eventually get higher yields. However, this potential of increased earnings depends on the structure of the value chain, number of actors and their market power, and the value added at its various value chain nodes. The more diverse and immediate the marketing possibilities of the farmers are, the higher their benefits can be. The potential socio-economic and societal benefits from investing in the improvement of TLV seed quality by eventually incorporating TLV seed into the formal seed market crucially depends on the potential contributions which these indigenous food products can have towards the income of the stakeholders in the value chain on the one hand and the benefits for food security and the healthy nutrition of the consumers on the other. Most commercial seed companies consider the TLV seed production as uneconomic. Across Africa, TLV seed is marketed informally, with less than $10 \%$ of seed planted being purchased from the formal markets. Few seed companies have committed themselves to the TLV seed production, for example in Kenya and Tanzania farmers have benefited from the involvement of big seed companies with many of them changing from using own farm saved seed to certified seed. Switching from own saved to certified seed increased the farmers market gross value by $213 \%$ between the period of 2001 and 2006 (Irungu et al., 2011). 
During the period of vegetable shortages, particularly during the dry seasons throughout the year, dried and processed TLVs become very important in household food security. Their abundance normally coincides with the peak of rain season, making it difficult to preserve them well and for larger quantities to be marketed throughout the relish gap. TLVs, like some of the modernized horticultural crops, are highly perishable. They are produced by resourcepoor rural sectors, who cannot afford the modernized preservation methods like refrigerating. The production of TLVs is relatively low especially during the dry seasons, meaning that the TLV market niche during this period can only be filled by the farmers using an irrigation mechanism. However, the quality of the processed TLVs is poor in some cases, can result in negative perceptions by the young generation, elite, and urban dwellers.

If the quality of the processed TLVs improves through preserving and turning them into products like cakes or flour, such products could increase the TLVs consumption by the young, elite, and urban dwellers. Also, the creation of awareness for the preparation or cooking of TLVs through cooking recipes can effectively and positively increase their consumption, particularly among the young, elite, and urban dwellers. Although the TLVs have the potential to facilitate the current imperative to foster food sovereignty and strengthen several of its components, this potential is not realized because they are classified as minor crops. 


\subsection{Solutions and Recommendations}

The availability of certified quality seeds coupled with the developed seed systems will enhance the production of TLVs. For example, Asfaw et al. (2012) reported better incomes were realized by smallholder farmers who adopted improved or certified seed varieties. The authors recommend the international organization such as the World Vegetable Center, already working in Eastern Africa to extend working with other countries in Southern and Western Africa to ensure quality seed production and develop TLV seed systems.

Different recipes and preparation methods that are passed through generations have also been promoted in Tanzania and should be scaled out in other countries in the continent to create awareness among the elite, young, and urban dwellers. For example, the World Vegetable Center is promoting consumption of TLVs and processing them into other products such as cakes and flour (Ochieng et al., 2016). The authors recommend increase in production and processing of traditional leafy vegetables to enhance their consumption by the elite groups and children.

The potential of traditional leafy vegetables to improve food and nutrition security TLVs contribute significantly to food security in the dry regions of Zimbabwe, as they are consumed by more than $60 \%$ of the households in these regions (Mpala et al., 2013); about $21 \%$ and $30 \%$ of the produced indigenous vegetables are consumed at home in Malawi and Mozambique (Chagomoka et al., 2014). TLVs account for about $80 \%$ of the total food use during winter in South Africa. Another key important issue regarding food security: the cash obtained from selling TLVs contributes significantly to food security at the household level, as it can be used to buy other food related items. In Zimbabwe, the rural poor people rely on TLVs for a period of nine months (five months of the rainy season and four of the dry months). They are prime sources of minerals and are naturally rich in nutrients, i.e. iron, protein, vitamins, and other micronutrients needed by the human body for healthy growth. They have the potential to help address micronutrient deficiency problems in many African countries.

Some TLVs have relatively high levels of nutrients, e.g. cleome and cowpea leaves contain more than $4 \mathrm{~g}$ ( $4.5 \mathrm{~g}$ and $4.4 \mathrm{~g}$, respectively) of protein and $275 \mathrm{mg}$ and $186 \mathrm{mg}$ of calcium, 
respectively, while sweet potato and cleome have $3.9 \mathrm{mg}$ and $2.2 \mathrm{mg}$ of iron, respectively, and cowpea leaves and cleome have $75 \mathrm{mg}$ and $113 \mathrm{mg}$ of Vitamin C, respectively per $100 \mathrm{~g}$ edible portion (Yang \& Keding, 2009). If incorporated in the daily diets of people in Africa, this new diet can be a key step in reducing malnutrition and food security problems faced by the continent. Most of the nutrients found in TLVs are very important, especially for the poor population who consumes mostly staples and tubers with little or no meat. Khoury et al. (2014) reported a global change in the dietary patterns, people are consuming calories, protein and fat, relying increasingly on wheat, maize and soybeans, along with meat and dairy products, showing increasingly shift towards monotonous and homogenous diets. TLVs can serve both purposes, as vegetables and as a supplement to starchy foods, as some TLVs, such as sweet potato and cassava roots, provide much needed energy as well, break the increasingly relying on monotonous diets.

TLVs are produced locally; farmers have control over seed and they can be quickly supplied to consumers, which ultimately supports domestic marketing structures in developing countries. Their marketing supports the flow of purchasing power from more affluent urban regions to rural areas. The development of TLV markets within African cities and other major urban centers could be considered a milestone in the re-introduction of underutilized local food crops for food security, food sovereignty, on-farm biodiversity conservation as well as halting biodiversity losses alluded to in SDG 15. Precious genetic variability of these domesticated plants, traditional foods and farming customs in developing countries can be preserved. From an agronomic perspective, TLVs might yield general beneficial effects for the cropping pattern and for fighting crop diseases.

\section{Potential socio-economic benefits for national food supply chains}

TLVs do not have high needs in terms of farming practices, production inputs, and weather conditions as they produce seed under the tropical condition unlike the exotic vegetables. This opens up the potential for farmers to easily expand TLVs production and obtain higher incomes through increased marketing of TLV seed and of TLVs. TLVs therefore possess a substantial potential for sustainably improving the livelihoods of the poor rural population and provision of food for the poor urban dwellers. 
The contribution of TLVs to household incomes have been reported to be higher than $20 \%$ in Malawi and Mozambique (Chagomoka et al., 2014). Besides income benefits, the production and trading of TLVs has been associated with women. In Zimbabwe and Kenya, the majority of the TLV traders and producers were women (60\% and $65 \%$ of the sample, respectively). This proportion confirms the trend shown in other reports. Mpala et al. (2013) reported that most TLV traders are women. Women are predominant in the production and marketing of TLVs, accounting for $57 \%$ and $95 \%$, respectively. Meaning that the women are empowered and therefore financially independent, consequently reducing their vulnerability. The incomes earned is very important, as it can be used for buying food for their families, provide housing and secure health care for the families among others (Schippers, 2000).

\section{Potential socio-economic benefits for rural areas}

Only in the late 1990s health institutions increased awareness designed to reduce the diseases of the affluent (i.e. diabetes, high blood pressure, and ulcers, among others) (Irungu et al., 2011). These ailments were predominantly associated with people dwelling in urban areas because of their unhealthy food consumption pattern. Whereas for their counterparts in rural areas, many diseases caused by food insecurity (such as malnutrition) have been associated with an unbalanced diet. But note that other patients who suffer from HIV/AIDS could benefit from TLVs, as they are said to mitigate this type of ailment (Irungu et al., 2011).

For years, the rural people valued the TLVs for food security as well as medicinal, social, cultural, and income generating item (Mpala et al., 2013). TLVs are important sources of essential macro- and micro-nutrients, hence their potential to reduce diseases of the affluent and diseases resulting from food insecurity. They are also important dietary components which are used to prepare sauces and relishes that accompany carbohydrate staples. Beyond their fundamental subsistence use, TLVs have the potential to address some of the Sustainable Development Goals (SDGs) that pertain to poverty, health, food and nutrition security in Africa.

Moreover, TLVs represent an inexpensive food of high and beneficial nutrient content (Schippers, 2000). With TLVs, a one-sided nutrition, especially for the poorer parts of the population, can be avoided by boosting this kind of crop production. In urban markets, TLVs 
command relatively better prices than the exotic vegetables and can be effectively produced by rural resource-limited farmers, hence the potential to foster rural development.

The potential of traditional leafy vegetables to contribute to the Sustainable Development Goals

Recent food price volatility has resulted in poverty and food insecurity in many developing countries, in particular Africa. The leaders from various countries created the MDGs to address some of the challenges, including poverty reduction and food insecurity problems Several countries managed to reduce the number of the population deemed poor by half. Following the successful implementation of the MDGs, the leaders of various countries created a new set of goals "Sustainable Development Goals", aimed to end poverty and hunger by 2030 .

TLVs have high nutritional values that can overcome malnutrition problems faced by Africa that still persist due to the lack of diversified diets. The consumption of TLVs has potential health benefits because they are rich in naturally inherent nutrients. TLVs can therefore contribute to the SDG 3 aimed at maintaining good health. These vegetables are resilient to adverse conditions prevailing in Africa. They can therefore provide food for many people across the continent, a trait making them excellent contributors to SDGs 1 and 2.

TLVs help smallholder farmers to diversify their limited income sources and provide an important source of employment in rural areas. TLVs command relatively high prices on the markets, giving them the potential to relieve rural poverty, in particular, for the vulnerable group, females. In most parts of Africa, TLVs are regarded as women crops, therefore, supporting their production and use can go a long way in the economic empowering of women, which in turn reduces the vulnerability of women and addresses gender inequality, as enshrined in SDG 5. Traditionally, African women are responsible for household food availability, preparation, and preservation. Women's involvement in the TLVs marketing is significantly high. Indigenoveg survey in Weinberger \& Pichop (2009) reported that women play a central role in the production, peddling and marketing of TLVs. They therefore offer a convenient pillar for lifting the women higher. Women dominate $100 \%$ in the retail markets and middle actors in Cote d'Ivoire, $68 \%$ in Uganda, $90 \%$ in Kenya, 65 \% in Tanzania, and $80 \%$ 
in South Africa (Indigenoveg in Weinberger and Pichop (2009)). These vegetables, therefore, form a crucial part of the female existence. TLVs have the potential to transform Africa's economy due to the high turnover and its effect on the income of women, as they play significant roles in the production and marketing of TLVs across Africa.

Currently some countries are supplementing the vegetable demand shortfalls through importing vegetables from neighbouring countries. For example, it is worth mentioning that Botswana and Zimbabwe have an import/export relationship. The local broadcasting company in Zimbabwe reported that Zimbabwe has spent more than 15 million USD in importing vegetables and citrus commodities in the first two months of 2016. Similarly, vegetables in Botswana are mostly imported from the neighbouring South Africa. Thus, limited foreign exchange is used to purchase products which could be supplied locally. The promotion of production, marketing and consumption of TLVs enhances local markets, curbing the externalization of much needed foreign currency. It would also reduce enrichment of the richer nations enshrined in SDG number 10.

Nevertheless, TLVs can contribute to the environment through the reduction of biodiversity loss, enshrined in SDG 15. Some of the TLVs types, like the cowpea, can easily adapt to poor soils, making them useful staple crops for marginalized areas. They can be incorporated in the production of crops by farmers living in areas marked by water scarcity and prone to hot temperatures caused by climatic change. In addition, some TLVs are deep rooted, hence they help to stabilize the soils. They also grow faster and provide canopy cover to the soil, reducing soil erosions and loss of soil moisture.

TLVs, therefore, have the potential to contribute to the Sustainable Development Goals 1, 2, $3,5,10,12,13$, and 15 (UNDP, 2016). Biodiversity loss reduction, good health maintenance, and the reduction of poverty and hunger can be strongly achieved through activities and policies promoting the production and use of TLVs. They are traded locally, supporting purchasing power from more affluent urban regions to rural areas and involve women command in the supply chain. Many people derive their income through various activities along the supply chain. They are sources of micronutrients required by the human body, food sources during crop failures. African national food and health policies should spearhead the 
production as well as promote their consumption to help address food insecurity and micronutrient deficiency problems in Africa. The national programmes are key in the implementation of such policies. A good example is the Malawi agricultural input programme, from which the country succeeded in feeding its people in a short period of time. The Green Revolution in India also transformed the agriculture; the country produced surplus and ended up exporting food. 


\subsection{Future research directions}

The outcome of our chapter noted that relatively few countries in Africa have soundly developed TLV seed systems and supply chains, which incentivize the actors along the TLV chains, resulting in the limited investment towards research. If the quality of TLV seed and distribution would be improved, then actors might benefit, realizing that more profits can also attract investors and researchers. Such successes can only be realized if Africa regions work collectively to improve the seed systems and supply chains. In particular, countries which already have seed policies in place should collaborate with the ones lagging behind, in order to improve the quality and accessibility of good quality seed by TLVs farmers. More research on determining the quality of TLV seed would contribute to availability of high quality seed on the markets. Equally important, researches aimed at understanding how the TLV supply chains are organized, improved seed distribution channels considering TLVs are increasingly marketed in cities across Africa. Such researches could provide the basis of improved TLVs supplies across Africa.

There is still a lack of knowledge about the methods of preserving and drying TLVs. The presence of contaminants, like molds or gritty-due fine sands, make TLVs at times unpalatable, due to abnormal smells. Much more attention needs to be given to improving the drying, preserving and processing of the TLVs into products. Some TLV products such as cakes or flour may attract the elite and urban dwellers to consume them. Besides, more effort needs to be channeled towards how to prepare or cook these vegetables. Most of the elite and urban dwellers lack the knowledge on how to prepare TLVs. Preparation or cooking of TLVs awareness creation through cooking recipes may effectively and positively increase their consumption, especially by the elite and urban dwellers. Research on post-harvest practices is needed. 


\subsection{Conclusions}

This chapter demonstrates the potential importance of TLVs in transforming the consumers' health and increasing farmers' incomes, particularly women as well as contributing to food security and the economies of African countries. TLVs have increasingly become crops of economic importance and their production cannot only feed an increasing and undernourished population, but also make people living in the marginalized areas more productive, given that TLVs grow well even in such stressful environments. Besides offering a greater opportunity to utilize the marginal areas, these vegetables help in diversification of the food basket and breaks the monotonous diets, which is commonly missing in the daily diets of many households in Africa.

There is minimal attention channeled towards these vegetables, yet they could quickly contribute reduction of the environmental biodiversity losses as well as contributing to a better food and nutrition security. In addition, relative to other field crops like maize, a conventional bred exotic vegetable, TLVs have received little attention in terms of research and funding from governments and international organizations. Just a few countries have put in place policies on TLV seed production, marketing and distribution.

Nevertheless, TLVs are now getting into the cities, showing an increase in demand for both vegetables and seed. The TLV seed systems in Africa are undeveloped, depriving the farmers of a variety of vegetables, as seed cannot be moved from one region to another. Commercial seed companies also shun the multiplication of TLV seed because they are considered to be less economically viable.

The seasonality and high perishability of these vegetables reduce their potential to be an important tool for addressing food and nutrition insecurity in Africa. In some countries, the urban dwellers and elite consumers still have negative perceptions of TLVs, considering them as food for poor people. Researchers need to prepare tasty and appetizing TLV dishes that can entice the young generation to consume them as well as promote consumer awareness campaigns concerning the nutritional benefits of eating TLVs. 
The supply chains of TLVs is not organized in many countries with exception of Kenya and Tanzania where farmers are benefiting from organized markets. In these countries, TLVs production and marketing has been transformed to be commercial and small-scale business entities and changing the livelihoods of many households. For example, in Kenya seed farmers under contract jointly produce seed of various TLV type with a total gross income of half a million USD with farmers earning on average almost 5,000 USD.

The women involvement in all activities along the TLV supply chain reduces women vulnerability and increases the women empowerment potential. TLVs have the potential to contribute to a number of SDGs, as they are traded locally, their consumption supports purchasing power from more affluent urban regions to rural areas, their market involves women command in the supply chain, their production, marketing and selling involve many people deriving their income through various activities along the supply chain, they are resources of micronutrients required by human body, they can considered as an option for food during crop failures and their increase in production and consumption will reduce biodiversity.

Smallholder farmers particularly, women who engage in production and marketing TLV, would be more financially empowered. However, the upscaling of these national successes to the entire Africa will be challenging because of the different national perceptions among policy makers.

\section{Acknowledgements}

This research was possible through the support of the Dutch Government through the Netherlands Fellowship Programme. The authors would like to acknowledge Professor Vasily Erokhin and two anonymous reviewers for their valuable comments. Thank you to all stakeholders involved in the traditional leafy vegetables for sharing with us your data. 


\section{References}

Ambrose-Oji, B. (2009). Urban Food Systems and African Indigenous Vegetables: Defining the Spaces and Places for African Indigenous Vegetables in Urban and Peri-Urban Agriculture. In C. M. Shackleton, M. W. Pasquini, \& A. W. Drescher (Eds.), African Indigenous Vegetables in Urban Agriculture (pp. 1-33). London: Earthscan.

Asfaw, S., Shiferaw, B., Simtowe, F. \& Lipper, L. (2012). Impact of modern agricultural technologies on smallholder welfare: Evidence from Tanzania and Ethiopia. Food Policy. 37: 283-295.

Bioversity International. (n.d.). Nutritious Underutilized Species. Retrieved February 15, 2017, from http://www.bioversityinternational.org//Nutritious_underutilized_species__Amaranth_1682.pdf.

Cernansky, R. (2015). Super Vegetables Long Overlooked in Parts of Africa, Indigenous Greens Are Now Capturing Attention for their Nutritional and Environmental Benefits. Nature. 522: 146-148.

Chagomoka, T., Afari-Sefa, V. \& Pitoro, R. (2014). Value Chain Analysis of Traditional Vegetables from Malawi and Mozambique. International Food and Agribusiness Management Review. 17 (4) 59-86.

Food and Agriculture Organisation of the United Nations. (2015). The State of Food Insecurity in the World 2015. Meeting the 2015 International Hunger Targets: Taking Stock of Uneven Progress. Rome: Food and Agriculture Organisation of the United Nations.

Ihle, R., von Cramon-Taubadel, S. \& Zorya, S. (2009). Markov-switching Estimation of Spatial Maize Price Transmission Processes between Tanzania and Kenya. American Journal of Agricultural Economics. 91 (5): 1432-1439.

Irungu, C., Mburu, J., Maundu, P, Grum, M. \& Hoeschle-Zeledon, I. (2011). The Effect of Market Development On-farm Conservation of Diversity of African Leafy Vegetables around Nairobi. International Journal of Humanities and Social Science. 8 (1): 198207.

Khoury, C. K., Bjorkman, A. D., Dempewolf, H., Ramirez-Villegas, J., Guarino, L., Jarvis, A., Rieseberg, L. H. \& Struik, P.C. (2014). Increasing Homogeneity in Global Food Supplies and the Implications for Food Security. Sustainability Science Agricultural Sciences. 111 (11): 4001-4006. 
Luckmann, J., Ihle, R., Kleinwechter, U. \& Grethe, H. (2014). World Market Integration of Vietnamese Rice Markets during the 2008 Food Price Crisis. Food Security. The Science, Sociology and Economics of Food Production and Access to Food. 6 (6): 1-17. Maundu, P., Achigan-Dako, E. \& Morimoto, Y. (2009). Biodiversity of African Vegetables. In C. M. Shackleton, M.W. Pasquini, \& A.W. Drescher (Eds.), African Indigenous Vegetables in Urban Agriculture (pp. 65-104). London: Earthscan.

Mpala, C., Dlamini, M., \& Sibanda, P. (2013). The Accessibility, Utilisation and Role of Indigenous Traditional Vegetables in Household Food Security in Rural Hwange District. International Open \& Distance Learning Journal International Research Conference Special Edition. 3 (1): 40-48.

Mumbi, K., Karanja, N., Njenga, M., Kamore, M., Achieng, C. \& Ngeli, P. (2006). Investigative Market Research: Viable Market Opportunities and Threats for Urban and Peri-Urban Farmers, Farm Concern International. Nairobi: Urban Harvest and International Potato Centre.

Nekesa, P. \& Meso, B. (1997). Traditional African Vegetables in Kenya: Production, Marketing and Utilization. In Proceedings of the IPGRI International Workshop on Genetic Resources of Traditional Vegetables in Africa: Conservation and Use. Rome: International Plant Genetic Resources Institute; Gatersleben: Institute of Plant Genetics and Crop Plant Research.

Ochieng, J., Afari-Sefa, V., Karanja, D., Kessy, R., Rajendran, S. \& Samali, S. (2016). How Promoting Consumption of Traditional African Vegetables Affects Household Nutrition Security in Tanzania. Renewable Agriculture and Food Systems. 1-11. doi: $10.1017 / \mathrm{S} 1742170516000508$.

Ojiewo, C., Tenkouano, A., Oluoch, M. \& Yang, R. (2010). The Role of AVRDC - the World Vegetable Centre in Vegetable Value Chains. African Journal of Horticultural Science. 3: 1-23.

Otsuka, K., Larson, D. F. \& Hazell, P. B. R. (2013). An Overview. In K. Otsuka \& D. F. Larson (Eds.), An African Green Revolution. Finding Ways to Boost Productivity of Small Farms (pp. 1-14). Amsterdam: Springer.

Pasquini, M. W., Assogba-Komlan, F., Voster, I., Shackleton, C. M., Abukutsa-Onyango, M. O. (2009). The production of African Indigenous Vegetables in urban and peri-urban agriculture: a comparative analysis of case studies from Benin, Kenya and South 
Africa. In C. M. Shackleton, M. W. Pasquini, \& A. W. Drescher (Eds.), African Indigenous Vegetables in Urban Agriculture (pp. 177-223). London: Earthscan.

Sasson, A. (2012). Food Security for Africa: An Urgent Global Challenge. Agriculture \& Food Security. 2 (1): 1-16.

Schippers, R. R. (2000). African Indigenous Vegetables. An Overview of the Cultivated Species. Chatham: Natural Resources Institute/ACP-EU Technical Centre of Agricultural and Rural Cooperation Book.

Shackleton, C. M., Pasquini, M. W. \& Drescher, A. W. (Eds.). (2009). African Indigenous Vegetables in Urban Agriculture. In C.M. Shackleton, M.W. Pasquini, \& A.W. Drescher (Eds.), African Indigenous Vegetables in Urban Agriculture (pp. 271-284). London: Earthscan.

United Nations Development Programme. (2016). Support to the Implementation of the 2030 Agenda for Sustainable Development. Retrieved July 27, 2016, from http://www.undp.org/content/undp/en/home/librarypage/sustainabledevelopment-goals/undp-support-to-the-implementation-of-the-2030-agenda/.

Veninga, W. \& Ihle, R. (in press). Import Vulnerability in the Middle East: Effects of the Arab Spring on Egyptian Wheat Trade. Food Security: The Science, Sociology and Economics of Food Production and Access to Food.

Weinberger, K. \& Msuya, J. (2004). Indigenous Vegetables in Tanzania- Significance and Prospects. Shanhua: AVRDC - The World Vegetable Center.

Weinberger, K. \& Pichop, G. N. (2009). Marketing of African Indigenous Vegetables along Urban and Peri-Urban Supply Chains in Sub-Saharan Africa. In C. M. Shackleton, M.W. Pasquini, \& A.W. Drescher (Eds.), African Indigenous Vegetables in Urban Agriculture (pp. 225-244). London: Earthscan.

World Vegetable Center. (2006). Empowering Small Scale and Women Farmers through Sustainable Production, Seed Supply and Marketing of African Indigenous Vegetables in Eastern Africa. Bangkok: AVRDC - The World Vegetable Center and Family Concern. Yang, R. \& Keding, G. B. (2009). Nutritional Contributions of Important African Indigenous Vegetables. In: C. M. Shackleton, M.W. Pasquini, \& A.W. Drescher (Eds.), African Indigenous Vegetables in Urban Agriculture (pp. 105-143). London: Earthscan. 
Annex

Additional reading

Afari-Sefa, V., Rajendran, S., Kessy, R. F., Karanja, D. K., Musebe, R., Samali, S. \& Makaranga, M. (2016). Impact of Nutritional Perceptions of Traditional African Vegetables on Farm Household Production Decisions: A Case Study of Smallholders in Tanzania. Experimental Agriculture. 52 (2): 300-313.

Afari-Sefa, V., Tenkouano, A., Ojiewo, C. O., Keatinge, J. D. H. \& Hughes, J. d’A. (2 0122 ). Vegetable Breeding in Africa: Constraints, Complexity and Contributions toward Achieving Food and Nutritional Security. Food Security: The Science, Sociology and Economics of Food Production and Access to Food. 4 (1): 115-127.

Durst, P. \& Bayasgalanbat, N. (Eds.). (2014). Promotion of Underutilized Indigenous Food Resources for Food Security and Nutrition in Asia and the Pacific. Bangkok: Food and Agriculture Organisation of the United Nations, Regional Office for Asia and the Pacific.

Key terms and definitions

Gross Margin Analysis: An analysis conducted between the revenue and the cost of producing TLVs, in our case per hectare per season.

Marketing Channels: A set of activities that necessitate the movement of the produced TLVs to the end user, i.e. the consumer.

Processors: Dehydrating the fresh vegetables from a perishable form into one that encourages a long shelf-life and thereby allows later usage.

Supply Chain: A sequential process involved in the TLVs production to the distribution of the produce. The supply chain for TLVs is relatively short because of the high perishability of the vegetables.

Sustainable Development Goals: Goals that aim at transforming our world through ending poverty and hunger, thereby achieving food security and improving nutrition and promoting sustainable agriculture.

Traders: Persons who engage in the buying and selling TLVs. 
Traditional Leafy Vegetables: Plants which originate on the African continent and have a century-long history of cultivation and domestication subject to regional agro-climatic conditions.

Traditional Leafy Vegetables Producers: Farmers that grow the TLVs. 


\section{General Discussion and Conclusions}

This chapter discusses the whole thesis and it consist of four sub-chapters which are elaborated below. The first sub-chapter gives a brief summary of all the core chapters of the thesis. The second sub-chapter integrates the findings from each chapter, discusses how they relate to each other, their implications and articulates how, in light of the findings from each chapter, the supply chain could be organised differently. The third sub-chapter discusses modes of data collection used as well as their influence on the data, limitations and recommendations for future research. The last sub-chapter gives brief insights into the potential options for future research.

\subsection{Research Objectives and Summary of Major Findings}

This PhD thesis analyses the supply chain of TLVs in Zimbabwe, and involves evaluating the quality of seeds, exploring the determinants of household consumption of and expenditure for TLVs in Zimbabwe and assessing the potential of TLVs for food security in Africa. The thesis is composed of four separate papers, presented in Chapters 2 through 5.

Chapter 2 aims at identifying the seed-borne pathogens of TLV seeds from five sources and establishing the relationship between seed infection and pathogen development in plants in the field. Laboratory and greenhouse experiments are used. Combining laboratory and greenhouse experiments enables further detection of the observed seed-borne pathogens and their pathogenicity to TLV species. This chapter shows the presence of seed-borne pathogens in both the seed and plants, ultimately confirming that the seed quality is poor. The quality strongly depends on the seed source. Training the farmers in seed production can significantly increase the production of quality seeds. Also, farmers may benefit by using recommended seed rates, get desired plant populations per unit area, as well as produce quality vegetables for the markets. The detection and identification of the seed-borne pathogens of TLV seeds in Zimbabwe enables the provision of quality seeds to farmers and reduces the spread of infections to new places. The literature lists observed seed-borne pathogens such as Alternaria spp., Xanthomonas campestris (Sharma et al., 1980; Bradbury, 1986; Grubben \& Denton, 2004) as important seed-borne pathogens of many plant species, including TLVs. 
Chapter 3 evaluates the germination of TLV seeds and determines the morphological characteristics of the seedlings from five sources. The five seed sources entail: Chinhoyi (Farmer 1), Murehwa (two sources: Farmer 2 and Farmer 3), Shamva (Farmer 4) and research organization (Farmer 5). They are located in three different agro-ecological regions of Zimbabwe (Mashonaland Central, Mashonaland West and Mashonaland East). Results show that Cleome gynandra seeds have the lowest percentage germination throughout the test intervals. Highest percentage germination was obtained for Bidens pilosa seeds. Similar results were reported by Ochuodho \& Modi (2007), who observed difficulties in seed germination of Cleome gynandra seeds. Similar to the seed testing methodology used in this study, the Cleome gynandra seeds were tested using International Seed Testing Association standards but had very low germination throughout the test intervals. These authors tested Cleome gynandra seeds at $20^{\circ} \mathrm{C}$ and germination was inhibited by light, resulting in $0 \%$ germination. However, it was found that pre-soaking Cleome gynandra seeds in water results in significantly higher percentage germination than observed in the potassium nitrate $\left(\mathrm{KNO}_{3}\right)$ treatment, which can be beneficial to farmers as they cannot afford the latter treatment. Bidens pilosa seeds have been found to show highest percentage germination confirming the findings of existing literature, e.g. Dube and Mujaju (2013). These authors reported the minimum germination standards to be $88 \%$. Across all the species, the percentage germination differed among the seed sources. The present results can be partially explained by the findings of Ochuodho \& Modi (2005) and de Barros et al. (2017), who confirmed that seed germination relates to the different habitats from which the seeds were collected. Amaranthus hybridus seeds have comparably moderate percentage germination with large variations among the seed sources, but higher percentage germination is obtained after longer storage periods. The low percentage germination obtained of Cleome gynandra seeds show very low productivity and farmers may face high production costs, as they have to purchase more seeds than required per unit area or face challenges in weeding as weeds will out-compete the sparsely populated crops.

Chapter 4 explores the determinants of household consumption of and expenditure for TLVs in Zimbabwe. The analysis is based on a survey collecting primary data in four districts and involves the use of linear regression models. The four districts are Harare, Makonde, Mbire 
and Binga. Several socio-economic characteristics of households, seasonal availability of and preferences for certain TLV types determine TLVs consumption and expenditure in Zimbabwe. The existing literature (e.g. Baker \& Wardle, 2003; Gido et al., 2017) shows that women consume more vegetables than men because women are more likely informed about the health benefits attached to vegetables. Spider flower (Cleome gynandra) is the TLV species most preferred in all regions and in both seasons, confirming Mukwereza's (2002) report that spider flower is one of the most preferred TLV species in Zimbabwe. The present findings show that the seasonality of supply starkly influences consumption and expenditure, beyond what other authors have investigated. People consume more fresh TLVs than dried ones, though dried TLVs play a significant role in the two poorest districts (Mbire and Binga), as depicted by larger quantities consumed during the dry seasons in these areas.

Ruel et al. (2005) reports that an enormous gap exists between the mean consumption of vegetables and WHO/FAO recommendations, clearly showing the need to address the consumption and production of vegetables in Africa. The lowest consumption of vegetables per capita is reported in the poorest communities (Ngugi et al., 2007), as they tend to consume more staples, tubers and little or no meat (Schippers, 2000). Households in Zimbabwe consume inadequate amounts of vegetables, with a daily intake of $30 \mathrm{~g}$ per capita, relatively far below the recommended levels for good health (FAO, 2015). The findings in Chapter 4 support the results of FAO (2015), showing very low consumption of vegetables per capita in Zimbabwe. This has exacerbated national health problems such as malnutrition and hunger.

Chapter 5 assesses the potential of TLVs in improving food security in Africa by combining insights from the literature and insights from the data analysis of Chapter 4. With higher margins and lower production costs, TLV production may be attractive to farmers. It shows that TLVs have the potential to play a major role in improving food security and facilitating food sovereignty across Africa. The production of TLVs across Africa is hindered by the unavailability of quality seeds while undeveloped seed systems limit farmers' choices. Moreover, the supply chain for TLVs in Zimbabwe is not organized, particularly seed production and markets, because TLV seeds are regarded as weeds in seed by-laws (Seeds Act, 1971). As a result, no quality standards for TLV seeds have been described. Moreover, 
the TLV seed markets have not been documented in Zimbabwe (Chisenga, [2016] personal communication). Unlike in Kenya and Tanzania, which have organized markets, the results show that the Zimbabwean TLV farmers are short-changed by the non-organized markets, as they remain restricted by the seasonality of their supply, among other constraints.

Chapter 5 also shows that TLV seeds distribution is localised, thereby denying the farmers new varieties of seed, cleaner and higher-quality seeds. However, localised distributions are advantageous to smallholder farmers as they support local developments, increase the accessibility of cheap seeds and ensure the timely availability of seeds. Although the quality of the seeds traded among the farmers is rarely known or documented, the gross margin analysis presented in Chapter 5 shows that TLV seeds and vegetables can be a lucrative business. The gross margin calculations have been based on farmers' perception of the costs required for production, which can be a realistic way of convincing them to use high quality seeds, or improved varieties they can achieve better income returns from their produce. Some TLV species, e.g. spider flower (Cleome gynandra), have relatively high market prices, providing smallholder farmers relatively high earnings.

The supply chain of TLV shows that all the seeds are shared, or exchanged amongst farmers before quality checks, risking disease build-up, low productivity and poor quality vegetables on the markets, suggesting that this supply chain needs to be organised differently. If improvements in the TLV seed system (e.g. realized by producing increasingly drought resistant varieties of the most popular TLVs) succeed in increasing the time span during which fresh TLVs are being marketed, then demand for fresh TLVs can be much better servedso that food and nutrition insecurity experienced in Zimbabwe may be effectively reduced. As consumption patterns in urban areas, where stakeholders are innovating TLV food chains, strongly differ from the ones in rural areas, the broadening of TLVs' marketing portfolio seems most promising which would also enable of benefitting from the higher urban purchasing power. This, in the end, might lead to the growth of the importance of the TLV supply chain in the domestic economy.

Very little attention has been paid to improving levels of production by innovative techniques at the input stages, like determining the quality of seeds before they are sold and pest control. 
By providing tested seeds with known quality to farmers, there will be significant increase in yield, reductions in storage losses of the vegetables, which, in turn, will benefit farmers and marketers, as well as consumers by means of increased returns and decrease in prices respectively. The findings from Chapter 4 shows that the most preferred mode of consuming TLVs is when they are fresh, indicating the necessity of including research, i.e. breeding programmes, in the supply chain. Breeding programmes working with actors on different segments of the supply chain may enhance the quality of TLVs and increase consumption. This could benefit the health of many and make the supply chain more robust and competitive, which may support the future growth of TLV markets in Zimbabwe.

From the gross margin analysis (Chapter 5), it can be concluded that selling seeds with known quality, improving the packaging of seeds (which were sold by the tablespoon, in pet coke lids, without packaging), packaging the dried vegetables (which were sold measured in cups, without proper packaging) results in higher prices on the market and therefore better returns to the farmers than selling those from unknown seed quality or unpackaged products. Considering agriculture is the backbone of the economies of many poor countries like Zimbabwe, growth in agriculture via the improved productivity of TLVs through research will go a long way in improving the national economies and contribute to poverty reduction. For example, a $10 \%$ increase in crop productivity leads to a reduction of $6-10 \%$ in people living on less than 1 USD per day (Irz et al., 2001).

This study shows it is beneficial to bring seed science and economics together in an interdisciplinary way to addressing the problems of TLVs. It is expected that farmers will find the use of saved seeds more attractive because they are considered cheaper than certified seeds, despite low yields (due to low plant population) and higher production costs (as at times they are compelled to use more seeds than required) compared to certified seeds (Chapters 2 and 3). At times farmers also do not have access to certified seeds. In spite of the benefits associated with cheaper saved seeds, the use of saved seeds may result in less income than that use of certified seeds. The gross margin calculations (Chapter 5 ) have been based on production costs reported by farmers, which are consistent with the farmers' economic model. In essence, if these farmers would have gone the extra mile by testing their seeds and improving packaging, they would be more likely to increase their incomes. For 
example, switching from their own saved seeds to certified seeds has been shown to increase farmers' market gross value by $213 \%$ during the period of 2001-2006 (Irungu et al., 2011).

Production of TLVs is increasingly being viewed as an important part of addressing food and nutrition insecurity and of improving the livelihoods of many poor people along the TLV supply chain in Zimbabwe. Chapter 4 shows that consumption of TLVs is no longer associated with the poor, and increased consumption by the elite and educated, young generations provides a significant opportunity for TLV producers to generate better revenue through commercial production. The demand for TLVs has increased, with consumers preferring fresh TLVs over dried. Based on the analytical framework, it is possible that before the seeds are distributed, exchanged or shared amongst the farmers, they could be tested; there is need to improve productivity through breeding or proper selection methods during harvesting, scouting and removing diseased plants during crop growth, thereby reducing disease buildup. Apart from improving production, this framework helped in this research to come up with a general assessment of TLV production and the problems associated with their production, their potential use in addressing food and nutrition insecurity and how to improve the livelihoods of people along the supply chain. The greater involvement of poor people in the TLV supply chain has an impact on incomes: better incomes can be realised through value addition and identification of alternative markets for products, i.e. exporting them. For example, in Chapter 5, the packaged dried TLVs in supermarkets were selling for more than 2 USD per $60 \mathrm{~g}$, compared to 0.50 USD for the same amount in local informal markets. There are several benefits to including the analytical framework. First, it is clear that the production constraints faced by farmers can be regarded a two-sided coin: on one side the use of poor quality seed, and the other side limited information on consumer preferences. Both reduce their chances of improved livelihoods through TLVs. 


\subsection{Conclusions and implications in traditional leafy vegetable supply chains}

Although the findings of this thesis (Chapters 2 and 3) show that TLV farmers use poor-quality seeds, the results of the gross margin analysis show the potential of TLV seed and vegetable markets. Even though the quality of TLV seeds on markets is in general not known and farmers risk problems in germination and seed degeneration which are all detrimental to crop production, there is a niche market for TLVs in Zimbabwe. In comparison, the results (Chapter 5) also show that prices for TLVs are higher than other vegetables, e.g. $60 \mathrm{~g}$ of dried spider flower goes for more than 2 USD in local supermarkets compared to a head of cabbage (around $1 \mathrm{~kg}$ ) selling for less than 1 USD. Synthesizing the findings from all chapters helps to understand the areas that need immediate attention towards increase consumption of TLVs in Zimbabwe, which can potentially be applicable to other regions of Sub-Saharan Africa as well.

The results for Chapters 2 and 3 show the seeds used by TLV farmers are of poor quality, due to the presence of seed-borne pathogens and low percentage germination. Across SubSaharan Africa, not only in Zimbabwe, only one-tenth of seed is of good quality, which is only available to farmers through formal seed markets; the remainder is made up by the farmers themselves from saved seeds or those obtained through exchange (Ngwerume \& Mvere, 2003; Adebooye et al., 2005). These latter seeds are of poor quality in most cases, characterized by low germination rates (Cernansky, 2015) and problems with purity (Karanja et al., 2012), among other quality attributes. This may also affect crop yields and the quality of their vegetables on the market (Biemond et al., 2012; Mancini \& Romanazzi, 2013; Ayana et al., 2014), reduce shelf life and product acceptance by consumers, and subsequently, reduce profits for farmers and the availability of the vegetables on the markets and, ultimately, reduce consumption as well. Seed-borne pathogens can prevent germination and cause serious threats to seedling establishment (Valkonen \& Koponen, 1990; Walcott, 2003) and plant vigour (Anjorin \& Mohammed, 2014), thereby reducing the yields and the quality of the product on the markets. Chapter 2 reports that farmers planting the seeds before testing them for seed-borne pathogens might be faced with seed degeneration across seed generations. 
The seasonal availability and supply can be a resultant of non-availability of seeds that give rapid germination and desired plant populations in the fields. Chapter 2 shows Cleome gynandra seeds carry many seed-borne pathogens that reduce plant health thus depressing the yields, shortening shelf life and reducing quality of the vegetables to the point that they become less acceptable to consumers. Also, the results obtained in Chapter 3 show that Amaranthus hybridus and Cleome gynandra seeds give better germination after prolonged storage, which become a challenge to smallholder farmers and can influence seasonality of the supply of these species on the market. Also, great challenges caused by low germination of Cleome gynandra seeds are encountered, posing again a major challenge to its producers. Taking into account the fact that spider flower has been reported to be one of the most popularly consumed TLVs in Zimbabwe (Chapter 4) and across Africa (Chapter 5), a considerable problem in germination can further decrease its consumption as well. Considering that the government of Zimbabwe has provided a major policy boost through the 'National Nutrition Strategy' (Tshuma, 2015) that seeks to address the national problems of food and nutrition, the production and consumption of TLVs can significantly contribute to attaining its goals. Seed quality is a key determinant of germination, plant vigour, even crop stand, and subsequently, yield and food security.

The use of poor-quality seeds by TLV farmers depresses yield and the quality of the product. For instance, some bacterial infections may cause blemishes (e.g. bacterial spots on harvested leaves) on the product which may not be acceptable to consumers. The shelf life of products may be reduced as well. Moreover, seed production is mostly at a small-scale level, with no seed certification attached, hence its economic value remains unknown. Seed certification facilitates seed trading nationally, regionally and internationally and also contributes to food and nutrition security as well as seed sovereignty. Farmers see very low profits as they fail to fetch premium prices on both vegetables and seed markets (Cromwell et al., 1992), because their seeds have not gone through any certification process and the resulting produce may be of poor quality. Furthermore, seed markets remain localised as the seeds do not have quality declaration documentation, i.e. seed testing or phytosanitary certificates, a normal requirement for international and regional seed trade. Keatinge et al. (2009) show that many smallholder farmers are actually willing to pay substantial amounts for good-quality seed. On the other hand, obtaining more expensive seed will be a challenge for poor, vulnerable 
farmers without adequate income, but their production costs could be compensated by high yields. Moreover, given the fact that TLVs are open pollinated species, they can subsequently reproduce the higher quality seed themselves. The study finds that all smallholder farmers are selling their seeds in local or Harare markets (Chapter 5 ). In both cases, the quality of all seeds remains unknown, risking poor crop stands in the fields, disease build-up or transfer of diseases to new places.

This thesis reports very low germination on Cleome gynandra seeds (Chapter 3). The results show, besides the environmental effects, the high occurrence of seed-borne pathogens on Cleome gynandra seed may have influenced the germination, partially confirming the reports of Broadbent et al. (1977); Agarwal \& Sinclair (1996); and Rude et al. (1999). They found that many seed-borne pathogens including Bacillus spp. and Alternaria spp. greatly reduce germination and seedling growth. Other studies have shown that some plant diseases affect plants in the field during crop growth and may also affect the harvested produce during storage as well as in the supply chain (Bhat et al., 2010; Ngadze, 2014), which may further influence the availability and quality of vegetables on the market. Notably, Gogo et al. (2017) reported losses of over $50 \%$ due to pests and diseases on TLVs. If these losses can be minimised, this may result in additional benefits to people's health and improve food security in Zimbabwe.

The Seeds Regulations and Seeds Acts of Zimbabwe apparently recognize TLV seed as weeds; as a result, less financial support is given to research to improve their productivity. The incorporation of TLVs in seed policies (e.g. coming up with seed certification standards for TLV seed) gives assurance to the farmers that seeds on the market are of adequate quality and improves the productivity of TLVs. Such developments ignite further research on seed quality and on the development of standard seed rates that enable TLV farmers to produce efficiently and effectively. They will then be able to meet desired plant populations per unit area and produce blemish-free products that can have extended shelf life. Economically, it is important to know the seed rate per unit area, as it reduces the unnecessary use of more seeds than required. Furthermore, knowing the quality of seed before sowing is crucial for the farmers as they would be able to treat the seeds before sowing. For example, a farmer may dress the seeds with a recommended fungicide before planting the crop, thereby reducing subsequent 
infections on plants and pressure on the environment. Already Zimbabwe is one of the countries in Africa with a well organised seed system; unfortunately, TLV seeds are left out of this seed system.

Chapter 4 shows that besides several socio-economic characteristics of households, seasonal availability and supply influence the consumption of and expenditure for TLVs in Zimbabwe. Households consume more fresh TLVs than dried ones. The problem with dried TLVs is that the nutritional quality decreases, drying methods differ and at times the quality becomes unacceptable to consumers (Mosha et al., 1997; Jansen van Rensburg et al., 2004). Chapter 5 reports the post-harvest challenges faced by smallholder farmers of TLVs, as the height of the production period coincides with the rainy season making it difficult to dry them. TLVs are highly perishable and seasonal, and during glut season a lot of vegetables are wasted due to lack of processing knowledge among smallholder farmers to make them available during the off-season. Relatively high humidity and overcast weather worsen the situation during the production peak because most dried TLVs tend to be mouldy and become less palatable and less suitable for human consumption (Mushonga, [2016] personal communication). Some dried TLVs become gritty after drying in open air; tiny dust particles settle on vegetables during the drying process, causing them to become less palatable (Mushonga, [2016] personal communication). Furthermore, food processing according to Mosha et al. (1997) reduces the nutrient content of TLVs. However, the results in Chapter 4 show the potential of dried TLVs for food and nutrition security in the two poorest dry districts of Zimbabwe (Mbire and Binga). Higher rates of consumption of dried TLVs have been observed in these districts. The higher values obtained in the two poorest dry districts show how important these vegetables are in these poor and dry communities. The findings support the findings of Vorster et al. (2007), who report that dried TLVs are major sources of food in the rural areas, especially during the winter season. Finding ways to improve seed germination and create rapid germination soon after harvest is necessary in order to increase the supply of fresh TLVs on the market; subsequently this may increase consumption. Also, preserving the nutritional quality after drying TLVs may increase their consumption.

The commercialisation of TLVs remains a challenge as it suffers from under-developed markets for both vegetables and seeds (Chapter 5). The smallholder farmers sell or exchange 
their seeds within their communities. Another routine practice is taking seeds to Harare and selling them in the public in the streets or at the Mbare-Musika farmers' market. Chapter 5 shows that the gross margins for various TLV seeds and vegetables are comparatively high, particularly considering that most of the seeds have not undergone any certification processes.

The underdeveloped markets for both vegetables and seeds pose the most substantial challenge (Chapter 5). The marketing of both vegetables and seeds varies across Sub-Saharan Africa. For example, the Seeds Rules and Regulations (1971) of Zimbabwe does not have guidelines for seed certification for these plant species, as they are only regarded as weeds (Seeds Act (1971)). On the contrary, in Zambia and Kenya there are seed companies already producing and marketing seeds of some TLV species, e.g. Cleome spp. (K' Opondo et al., 20005 ). But generally, in most Sub-Saharan African countries, the TLV seed markets are undeveloped, thereby limiting the movement of seeds within nations, regions and internationally, consequently limiting smallholder farmers' choices, access to new varieties and qualities of seed that they produce themselves. As a result, smallholder farmers get very low yields per unit area. The unavailability of phytosanitary measures for these crops impedes the movement of seeds. Testing the seeds before marketing can result in competitive prices for their seeds and enables the movement of seeds to other places.

Linking up seed production and TLVs, Chapter 4, results show that the most preferred TLV types across all the districts in the study is spider flower (Cleome gynandra), with consumers preferring fresh to dried. Although Cleome gynandra has a comparative advantage over other species, the farmers may face the challenge of very low productivity due to low crop stands in their fields. Notably, the very poor percentage germination observed in Chapter 3, together with the high occurrence of seed-borne pathogens reported in Chapter 2, implies a significant impediment to the commercialisation of spider flower. Cleome gynandra genotypes existing in Zimbabwe exhibit low germination and are not suitable for the commercial promotion of traditional leafy vegetable production, implying that farmers have to use more seed than required to compensate for low germination. Such developments are very uneconomic to the farmers, considering they are relatively poorly resourced smallholder farmers. Also considering the gradual germination of Cleome gynandra seed with age retards the consistent 
production, hence its marketing, creating seasonal supply. Farmers need seeds that germinate vigorously for them to achieve their desired plant population in the fields. With the low germination observed for Cleome gynandra seeds, farmers are faced with the challenge of covering the production gaps when seeds are dormant. Amaranthus hybridus seeds have significantly lower occurrence of seed-borne pathogens and show comparably moderate percentages of germination, which is good for the commercial promotion of their production. However, the morphological characteristics of the most frequently occurring observations vary, implying that the seeds may consist of admixtures, posing a risk of a tedious selective harvest by smallholder farmers. Such developments may lead to smallholder farmers incurring high labour costs for harvesting and sorting, or else they fail to meet consumer preferences and demands. Training seed producers in cultural maintenance of the crops, i.e. removing all off-types and diseased plants during crop growth, may reduce diseases and admixtures.

The high gross margins observed in Chapter 5 implies potentially competitive business for smallholder farmers producing TLVs and seeds. Considering that TLVs can be produced with minimum resources, if given the same opportunity as the Brassica species, TLVs can be equally important in addressing economic challenges, food and nutrition security in Zimbabwe. The monthly household expenditure on TLVs ranges from 6 USD to 14 USD per household. Also local programmes, including the National AIDS Council, Ministry of Health (with focus on pregnant and lactating mothers) and Zimbabwe Prisons Services are promoting the consumption of TLVs for health issues (Muusha, [2016] personal communication). Promotional campaigns addressing the consumption of TLVs across all the nation may result in greatly increased TLV consumption.

There are various actors along the TLV supply chain in Zimbabwe, including seed producers and marketers, and vegetable producers, marketers and processors. The main reason to include all the mentioned segments is that this thesis focuses on the supply chain of TLVs, particularly including research on seed quality. This research is chosen keeping in mind that production must be increased in order to meet demand. Various research projects have been done to increase consumption of TLVs, but if the seed quality is not evaluated and documented, there are likely to be extinctions due to over-harvesting. In Zimbabwe, TLVs are 
normally grown, then very tender leaves are harvested or young plants are uprooted. Such tendencies, if not carefully guarded, might result in the extinction of certain species. 


\subsection{Limitations and recommendations for future research}

This section identifies the limitations encountered during the research. Also, recommendations for future research are put forward.

The sample sizes for Chapters 2 and 3 are very small, given that only five farmers from three provinces of Zimbabwe are used, which may not be representative. Conducting research which has representative results for Zimbabwe as a whole, taking as many samples as possible from each province can make a big difference. It is also worthwhile to note that these three provinces are located in different agro-ecological regions (with varying temperatures and rainfall patterns), which might result in different sources of variation for the obtained results. Furthermore, the seed crops were grown, harvested and managed differently, which again might be a source of variation. However, the consistent trends observed among test intervals allows drawing the conclusion that the quality of seeds is partly influenced by its source. Future research replicating sites with one management system might give robust and reputable results. Such tests can be conducted at the research and development level. Validation of International Seed Testing Association (ISTA) guidelines on breaking dormancy in Cleome gynandra might enhance its germination capacity. Some authors argued that seeds sensitive to light are sensitive to $\mathrm{KNO}_{3}$ as well, but ISTA recommends the use of both light and $\mathrm{KNO}_{3}$ to break the dormancy in Cleome gynandra. The economic losses caused by pathogens on TLVs is hardly researched and documented. Reduced production and reduced shelf life (post-harvest losses) can be quantified. Post-harvest losses in three areas in Kenya were estimated by analysing responses to a survey from 45 TLV farmers from each location (Gogo et al., 2017). These authors observed that TLV farmers experienced losses ranging from $10 \%$ to over $50 \%$ on average, depending on location and supply stage. The losses can be further quantified and categorized accordingly, i.e. per hectare, yield losses, discarded vegetables, cost of pesticides, increased labour and decreased market value of the product.

A limitation of Chapter 4 is that the data used for the analysis is obtained during two seasons (on-season and off-season), using different households for each season, making it impossible to make significant comparison between seasons per household. There is a possibility that 
each household has its own preference depending on each season, which cannot be explained well by this research.

One main limitation for Chapter 5 is again the sample size, collected data from 22 key informants using the snowball sampling technique. The method is ideal for the Zimbabwean situation, where seed production and distribution is strictly controlled by the government. Unlike other crop seed like maize, wheat, TLV seed production is not documented, leaving the authors with no option but to use the snowball method to reach the unknown TLV seed growers and marketers. With other seed crops, the list of growers per year can be accessed at Zimbabwean Seed Services, which is the certifying authority and relevant arm of the government, custodian of all seed policies and regulation. In this case, the authors relied on the information on seed growers received from vegetable marketers or processors. Three provinces of Zimbabwe may not be representative of the ten total provinces constituting Zimbabwe. Instead, the data is collected on four segments of the supply chain, using at least five people per segment.

\section{Methodology}

Although less than 400 seeds (as per ISTA seed testing standards) are used for the tests (Chapters 2 and 3), repetitive tests and testing seeds using different media improved the efficiency and effectiveness of the methodology. The snowball sampling technique is used to solicit some of the data in Chapter 5. The chapter also combines insights from the literature and insights from the data analysis of Chapter 4. A characteristic of the TLV supply chain is that some actors are administratively invisible, e.g. seed producers and marketers are not documented (Chisenga, [2016] personal communication) and are difficult to find. The use of snowball sampling as a tool to gather data is found to be ideal for this survey. The authors' personal communication with one of the Registrar Officers at Seed Services noted that TLV seeds are regarded as weeds, hence their production and marketing are not documented. The data for the fourth chapter was collected through surveys administered by Agritex officers in respective areas, where a trust relationship had already been established with Agritex, thereby reducing the likelihood of people giving different answers, or at times lying. Initial pre-tests conducted on all study areas helped us to identify how the questions were 
likely to be interpreted. The pre-test surveys were conducted together with Agritex officers in respective areas. Also, the Agritex officers in respective areas were trained, reducing the chances of receiving different answers for a given question. Apart from all the above-listed techniques aimed at reducing inconsistences among enumerators, the data was collected using a structured questionnaire with most of the questions being closed, while some responses used codes; a Likert scale from 1 to 5, with 1 for strongly decrease/very unimportant/completely disagree/never and 5 for strongly increase/very important/completely agree/very frequently. Other studies have reported little dispute over the use of scales (e.g. Adams \& Cox, 2008). Snowball sampling contradicts other conventional sampling methods, because of the selection bias which in turn limits its usage in carrying out scientific research (Atkinson \& Flint, 2001; Baltar \& Brunet, 2012). This thesis is the first that studies a number of segments in the TLV supply chain and uses snowball sampling technique as an instrument for collecting data (Chapter 5's results are derived from this), which, for that matter, represents a great challenge in this research, due to bias. Therefore, data is collected on four different segments of the supply chain.

\subsection{Potential options for future research}

The reason for conducting repetitive research on different agro-ecological regions is that environmental conditions, which in turn may cause variation, may be explained. For example, trends of results per area can enable to safely draw certain conclusions. One of the ways of dealing with a limited sample size is conducting as many tests as possible; by gathering ample data, trends can offer better insights. TLVs could be included in the Zimbabwean Seed laws as insisted by Dube and Mujaju (2013). This could be achieved after conducting repetitive research, then deriving standards for each species by the assessing the trends of the results. Already in Zimbabwe, there are a number of programmes targeting certain groups of people, e.g. The National AIDS Programme, for the promotion of TLV consumption. If such promotional programmes aim at all people in the country, the consumption of TLVs may increase and nutritional challenges may be addressed. 


\section{References}

Adams, A., \& Cox, I. (2008). Questionnaires, in-depth interviews and focus groups. In P. Cairns \& A. L. Cox (Eds.), Research methods for human computer interaction (pp. 17-34). Cambridge: Cambridge University Press. 9780521870122 CUUK352-Cairns.

Adebooye, O. C., Ajayi, S. A., Baidu-Forson, J. J., \& Opabonde, J. T. (2005). Review: Seed constraint to cultivation and productivity of African indigenous leaf vegetables. African Journal of Biotechnology. 4 (13): 1480-1484.

Agarwal, V. K., \& Sinclair, J. B. (1996). Principles of Seed Pathology. $2^{\text {nd }}$ ed. Boca Raton, FL: CRC Press.

Anjorin, S. T., \& Mohammed, M. (2014). Effect of seed-borne fungi on germination and seedling vigour of watermelon (Citrullus lanatus thumb). African Journal of Plant Science. 8 (5): 232-236.

Atkinson, R., \& Flint, J. (2001). Accessing hidden and hard-to-reach populations: Snowball research strategies. Social Research Update (Department of Sociology, University of Surrey). 33: 1-4.

Ayana, A., Afari-Sefa, V., Emana, B., Dinssa, F. F., Balemi, T., \& Temesgen, M. (2014). Analysis of vegetable seed systems and implications for vegetable development in the humid tropics of Ethiopia. International Journal of Agriculture and Forestry. 4 (4): 325-337.

Baker, A. H., \& Wardle, J. (2003). Sex differences in fruit and vegetable intake in older adults. Appetite. 40: 269-275.

Baltar, F., \& Brunet, I. (2012). Social research 2.0: Virtual snowball sampling method using Facebook. Internet Research. 22 (1): 57-74.

Bhat, K. A., Masood, S. D., Bhat, N. A., Bhat, M. A., Razvi, S. M., Mir, M. R., Akhtar, S., Wani, N., \& Habib, M. (2010). Current status of post-harvest soft rot in vegetables: A review. Asian Journal of Plant Sciences. 9: 200-208.

Biemond, P. C., Stomph, T. J., Kamara, A., Abdoulaye, T., Herane, S., \& Struik, P. C. (2012). Are investments in an informal seed system for cowpeas a worthwhile endeavour? International Journal of Plant Production. 6 (3): 367-386.

Bradbury, J. F. (1986). Guide to plant pathogenic bacteria. Wallingford, UK: CAB International Mycological Institute. 
Broadbent, P., Baker, K. F., Franks, N., \& Holland, J. (1977). Effect of Bacillus spp. on increased growth of seedlings in steamed and in non-treated soil. Phytopathology. 67: 10271034.

Cernansky, R. (2015). Super vegetables long overlooked in parts of Africa, indigenous greens are now capturing attention for their nutritional and environmental benefits. Nature. 522: $146-148$.

Cromwell, E., Esbern, F. H., \& Turner, M. (1992). The seed sector in developing countries: A framework for performance analysis. Working Paper 65. London: Overseas Development Institute (ODI).

de Barros, R. T., Martins, C. C., da Silva, G. Z., \& Martins, D. (2017). Origin and temperature on the germination of beggartick seeds. Revista Brasileira de Engenharia Agrícola e Ambiental. 21 (7): 448-453.

Dube, P., \& Mujaju, C. (2013). Determination of standards for purity and germination for African indigenous vegetable (AIV), blackjack (Bidens pilosa). Advanced Journal of Agricultural Research. 1 (003): 32-38.

Food and Agriculture Organization of the United Nations (FAO) (2015). The state of food insecurity in the world 2015: Meeting the 2015 international hunger targets; Taking stock of uneven progress. Rome: Food and Agriculture Organization of the United Nations.

Gido, E. O., Ayuya, O. I., Owuor, G., \& Bokelmann, W. (2017). Consumer acceptance of leafy African indigenous vegetables: Comparison between rural and urban dwellers. International Journal of Vegetable Science. 23 (4): 346-361.

Gogo, E. O., Opiyo. A. M., Ulrichs, C. H., \& Huyskens-Keil, S. (2017). Nutritional and Economic postharvest loss analysis of African indigenous leafy vegetables along the supply chain in Kenya. Postharvest Biology \& Technology. 130: 39-47.

Grubben, G. J. H., \& Denton, O. A. (Eds.) (2004). Plant resources of tropical Africa 2: Vegetables. Wageningen: PROTA Foundation; Leiden: Backhuys Publishers.

Irungu, C., Mburu, J., Maundu, P., Grum, M., \& Hoeschle-Zeledon, I. (2011). The effect of market development on-farm conservation of diversity of African leafy vegetables around Nairobi. International Journal of Humanities and Social Science. 8 (1): 198-207.

Irz, X., Lin, L., Thirtle, C., \& Wiggins, S. (2001). Agricultural productivity growth and poverty alleviation. Development Policy Review. 19: 449-466. 
Jansen van Rensburg, W. S., Venter, S. L., Netshiluvhi, T. R., Heever, E., van den Vorster, H. J., \& de Ronde, J. A. (2004) Role of indigenous leafy vegetables in combating hunger and malnutrition. South African Journal of Botany. 70 (1): 52-29.

Karanja, D., Okoko, N., Kiptarus, E., Okongo, P., Samali, S., Katunzi, A., Mtwaenzi, H., Mwakitwang, F., Afari-Sefa, V., Musebe, R., Kimani, M., \& Kimenye, L. (2010). Promoting farmer-led seed enterprises of African indigenous vegetables to boost household incomes and nutrition in Kenya and Tanzania. Paper presented at the Conference on Agricultural Biodiversity in Africa. Proceedings, pp. 1-13. http://www.asareca.org/publication/promoting-farmer-led-seed-enterprises-africanindigenousvegetables-boost-household.

Keatinge, J. D. H., Hughes, J. d’A., Tenkouano, A., Hamilton, K., Easdown, W. J., \& Mongi, H. O. (2009). Vegetables and small private-sector interests. In A. G. Brown (Ed.), World Food Security: Can private sector $R \& D$ feed the poor? (pp. 60-68). Proceedings, Crawford Fund for International Agricultural Research, Canberra, 27-28 October.

K' Opondo, F. B. O., Muasya, R. M. \& Kiplagat, O. K. ( $\left(\begin{array}{llll}2 & 0 & 0 & 5\end{array}\right)$. A review on the seed production and handling of indigenous vegetables (spider plant, jute mallow and African nightshade complex). In M. O. Abukutsa-Onyango, A. N. Muriithi, V. E. Anjichi, K. Ngamau, S. G. Agong, A. Fricke, B. Hau \& H. Stutzel (Eds.), Proceedings of the third Horticulture Workshop on Sustainable Horticultural production in the Tropics (pp. 4248). Maseno: Maseno University.

Mancini, V., \& Romanazzi, G. (2013). Seed treatments to control seedborne fungal pathogens of vegetable crops. Pest Management Science. 70: 860-868.

Mosha, T. C., Pace, R. D., Adeyeye, S., Laswai, H. S., \& Mtebe, K. (1997). Effect of traditional processing practices on the content of total carotenoid, beta-carotene, alphacarotene and vitamin A activity of selected Tanzanian vegetables. Plant Food Human Nutrition Journal. 50 (3): 189-201.

Mukwereza, L. ( $\left.\begin{array}{llll}2 & 0 & 0 & 2\end{array}\right)$. Report on the study on processed indigenous vegetables (IV's): Mutare city and its peri-urban areas. A study undertaken as part of activities for the CPHPfunded IV's project. Agricultural Management Services, Harare, Zimbabwe. https://assets.publishing.service.gov.uk/media/R7487i.pdf.

Ngadze, E. (2014). In vitro and greenhouse evaluation of botanical extracts for antifungal activity against Phytophthora infestans. Journal of Biopesticides. 7 (2): 198-203. 
Ngugi, I. K., Gitau, R., \& Nyoro, J. K. (2006). Access to high value markets by smallholder farmers of African Indigenous Vegetables in Kenya. Regoverning Markets Innovative Practice series. London: Sustainable Markets Group.

Ngwerume, F. C., \& Mvere, B. (2003). Report on the findings of a socio-economic survey on the marketing, consumption and production of traditional vegetables in the urban and peri-urban areas of Harare, Zimbabwe. DR\& SS-HRC/NRI project a0892.

Ochieng, J., Afari-Sefa, V., Karanja, D. R., Srinivasulu, S., \& Samali Kessy, R. (2016). Promoting consumption of traditional African vegetables and its effect on food and nutrition security in Tanzania. Invited paper presented at the 5th International Conference of the African Association of Agricultural Economists, Addis Ababa, Ethiopia, 23-26 September.

Ochuodho, J. O., \& Modi, A. T. (2005). Temperature and light requirements for the germination of Cleome gynandra seeds. South African Journal of Plant and Soil. 22 (1): 49-54.

Ochuodho, J. O., \& Modi, A. T. (2007). Light-induced transient dormancy in Cleome gynandra L. seeds. African Journal of Agricultural Research. 2 (11): 587-591.

Ojiewo, C., Tenkouano, A., Oluoch, M., \& Yang, R. (2010). The role of AVRDC - The World Vegetable Centre in vegetable value chains. African Journal of Horticultural Science. 3: $1-23$.

Rude, S. V., Duczek, L. J., \& Seidle, E. (1999). The effect of Alternaria brassicae, Alternaria raphani and Alternaria alternata on seed germination of Brassica rapa canola. Seed Science and Technology. 27: 795-798.

Ruel, M. T., Minot, N., \& Smith, L. (2005). Patterns and determinants of fruit and vegetable consumption in Sub-Saharan African: A multicountry comparison. Washington, DC: World Health Organization International Food Policy Research Institute.

Schippers, R. R. (2000). African indigenous vegetables: An overview of the cultivated species. Natural Resources Institute/ACP-EU Technical Centre for Agricultural and Rural Cooperation. Chatham: Natural Resources Institute, University of Greenwich.

Seeds Act, [Chapter 19:13] (1971). Print flow, Government printers, Harare, Zimbabwe. http://www.parlzim.gov.zw/attachments/article/105/SEEDS_ACT_19_13.pdf.

Seeds Rules and Regulations (1971). Print flow, Government printers, Harare, Zimbabwe. 
Seeds [Certification Scheme] Notice (2000). Print flow, Government printers, Harare, Zimbabwe.

Sharma, A. D., Tnakur, V., \& Munjal, R. L. (1980). Seed myeoflora of Amaranthus eaudatus, its pathology and control. Indian Phytopathology. 33: 242-244.

Tshuma, N. VP Mnangagwa launches National Nutrition Strategy. Chronicle, 30 April 2015. http://www.chronicle.co.zw/vp-mnangagwa-launches-national-nutrition-strategy.

Valkonen, J. P. T., \& Koponen, H. (1990). Seed-borne fungi of Chinese cabbage (Brassica pekinensis), their pathogenicity and control. Plant Pathology. 39: 510-516.

Vorster, I. H. J., van Rensburg, W. J., Van Zijl, J., \& Venter, S.L. (2007). The importance of traditional leafy vegetables in South Africa. African Journal of Food, Agriculture, Nutrition and Development. 7 (4):1-13.

Walcott, R. R. (2003). Detection of seedborne pathogens. HortTechnology. 13 (1): 40-47.

Weinberger, K., \& Pichop, G. N. (2009). Marketing of African indigenous vegetables along urban and peri-urban supply chains in Sub-Saharan Africa. In C. M. Shackleton, M. W. Pasquini \& A. W. Drescher (Eds.), African indigenous vegetables in urban agriculture (pp. 225-244). London: Earthscan. 


\section{Summary}

TLVs are very important as sources of cheap nutrition for many people in Zimbabwe. Their cultivation and use are crucial for food and nutrition security across the country, as they provide health and lean nutrition for both poor and rich people. This thesis analyses the supply chain of TLVs in Zimbabwe using an interdisciplinary approach. The supply chain of TLVs, particularly seed production and markets, is not as well organised as other seed types which follow the formal seed system. In the formal seed system, the production and marketing of seeds are controlled by the government (Seed Services) under two Acts of the Parliament (Seeds and Plant Breeders Rights Acts).

The production potential of TLVs can be increased by providing smallholder farmers with adequate high-quality seed, especially those free from seed-borne pathogens. Seed-borne pathogens are detrimental and an impediment to agriculture, particularly to those plant species that are neglected by regulations, i.e. TLVs. They have received little attention as far as seed health issues are concerned, risking tremendous seed degeneration over time. Unlike grains, seeds are shared within communities, regions and at times find their way into international markets, posing the risk of spreading pathogens to new places. Seed-borne pathogens are also responsible for weakening the seedlings, thereby reducing plant vigour, hence crop stand. Good crop stand is key to the commercialization of TLVs. Seed-borne pathogens also affect germination and the quality of products on the market. Taken altogether, checking the quality of the seeds before the seeds are sown is important for determining the economic and planting value and achieving the desired plant population per unit area. Although the findings do not directly show the economic and planting value of TLV seeds, the results (Chapter 5) indicate that farmers can potentially benefit from high-quality seed. This can be also be used to reward the farmers that have high-quality seed accordingly.

Seed-borne pathogen detection, seed germination evaluation, and two surveys on seed markets and household consumption of and expenditure for TLVs were carried out to answer four specific research objectives. Each specific research objective is a chapter. Chapter 2 assesses the quality of TLV seed. All the seeds shared or traded amongst farmers are infected by various pathogens, but the infestations vary in magnitude. High pathogen infestations were observed on Cleome gynandra seeds and specific seed sources (Farmers 1, 2, 3 and 4). 
Xanthomonas campestris, Fusarium oxysporum ${ }_{L}$ Alternaria alternata and Bacillus spp. isolates are pathogenic to Amaranthus hybridus, Cleome gynandra and Bidens pilosa plants, as confirmed by pathogenicity tests. All bacteria isolates give a positive response on all three TLV plant species and with varied magnitudes, ranking as either highly, moderately or weakly virulent pathogens, based on host responses. None of the plant species were found to possess high resistance to the present bacteria pathogens observed in the study. Furthermore, the occurrence of seed-borne pathogens on both the seeds and plants of Farmers 1, 2, 3 and 4 clearly showed how poor the quality of the seed was.

Chapter 3 deals with the second objective, 'Evaluation of the germination of traditional leafy vegetable seeds and determination of the morphological characters of the seedling from five sources'. This chapter evaluates the germination of traditional leafy vegetable seeds and determined the morphological characteristics of seedlings from five sources. Although the evaluation of germination in a variety of TLV seeds have been studied before, this study uses international seed testing standards, with tests conducted at regular intervals on five seed sources. The results showed variable germination capacities, with Cleome gynandra seeds depicting very low germination rates throughout the tests. Results showed that Cleome gynandra seeds germinated better after pre-soaking for 24 and 48 hours and in field conditions. Cleome gynandra and Amaranthus hybridus seeds showed better germination after prolonged storage, a phenomenon which may be disastrous for farmers who want to continuously produce TLVs. Farmers need seeds with rapid germination and vigorous plants in order to satisfy market demands. The heterogeneous population observed from Amaranthus hybridus seed indicated the rich genetic pool that can be used for breeding purposes and improvement of the species through selection at the farmers' level.

Chapter 4 examines the determinants of household demand for TLVs using primary data from four districts of differing food security statuses in Zimbabwe. Based on a multivariate linear regression model, it was found that households in poor districts consume and spend on average less on fresh TLVs, although they consume them more often in dried form. Seasonal availability and supply affect TLV prices and influence the frequency and amount of consumption. Consumers prefer fresh TLVs over dried. The results showed that besides seasonal availability and supply, there are other factors that influenced TLV consumption. 
These include socio-economic characteristics of households such as years of schooling, general health and geographical location. Moreover, this research compared the preferences amongst the four districts. The results showed that four TLV species play a significant role in Zimbabwean diets, with varying regional preferences. Spider flower (Cleome gynandra) emerged as the one of the most preferred TLV types overall.

Chapter 5 assesses the potential of TLV in improving food security in Africa by combining insights from the literature and insights from the data analysis of Chapter 4 . Based on the gross analysis, survey results and literature, it was found that TLVs have the potential to improve food security of the farmers, as they can be produced and sold cheaply, the money received can be used to buy other food stuffs and meet family needs, whilst some vegetables can be consumed within households.

The study developed an analytical framework. This framework helped in this research to come up with a general assessment of TLV production and the problems associated with their production, their potential use in addressing food and nutrition insecurity, and how to improve the livelihoods of people along the supply chain. 


\section{Acknowledgements}

I am indebted to the Dutch Government, through NUFFIC, for its financial support of my study programme leading to an award of PhD in Agricultural Economics and Rural Policy, specializing in the supply chain at Wageningen University. Furthermore, I am thankful to Professor Wim Heijman for granting me the opportunity to carry out my $\mathrm{PhD}$ thesis under his supervision in the Agricultural Economics and Rural Policy Group. I would like to thank Professor Paul Struik. I benefitted immensely from you in conducting this research which combined social and technical aspects. Special thanks to Dr Rico Ihle, I appreciate the endless time and effort you put in my work and your long hearted patience in taking me through this challenging journey. Accomplishing the research and this thesis as its final product was no one woman's hand, but a joint effort from you all, once again special thanks to you all!

I would like to thank, Karen and Marian, you made my stay and work comfortable. I also want to pay my gratitudes to my fellow PhD students in the Agricultural Economics and Rural Policy Chair and my fellow Zimbabweans for sharing PhD experiences together. I express my gratitude to you all for all forms of assistance while developing tools for data collection, conducting analyses and writing this PhD thesis. Next, I would like to thank Professor Justus Wesseler for the interesting social interactions during our usual group coffee break and your interest in knowing the PhD students' progresses. To me you were like my father, who always wanted to have very close interactions with me even I felt I was an adult. You filled that gap. I would like to acknowledge Professor Vasily Erokhin and various anonymous reviewers for their valuable comments. Thanks to the stakeholders involved in the traditional leafy vegetables sector for sharing with me your data and expertise. The permission of Agritex Principal Director Mr. Gondo to work with his staff is highly appreciated. I would like to thank the extension officers from Agritex for their substantial support in data gathering, University of Zimbabwe Technicians, Mrs Matikiti, Sanyamuwera Martin and Magadhi Denver, Seed Services staff for conducting the laboratory experiments. I would like to thank the Plant Protection - Pathology staff and Mrs Muhera for conducting pre-tests for my seed health paper. Thanks to all farmers involved in producing the seeds which I have used to conduct my research. Your timely participation during my research made my work a success. 
Last but not least, I extend my appreciation to my three lovely, wonderful sons, Zvikomborero, Tanaka and Tendai, my relatives and friends, in particular, Mrs Madamba, Alfred and Mrs Zhou. Your unwavering support and encouragement has taken me this far. To my sons, I say thank you. You always cheered me up, you were my sources of strength when the going got tough and at times contemplating if I would manage. 


\section{Biography}

Praxedis Dube was born on 1 January 1967 in Mberengwa district, Zimbabwe. After completing her secondary school, she started working as an Assistant Seed Analyst at Seed Services Institute in the Department of Research and Specialist Services under the Ministry of Agriculture, Mechanization and Irrigation Development. From August 2003 to July 2006, she enrolled with Women's University in Africa of Zimbabwe and received an honors in B. Sc. Agriculture, specializing in Horticulture. Her thesis was on evaluating the effects of harvesting injury on germination and seedling development on soya bean (Glycine max). In January 2009, Praxedis was awarded a Scholarship from the Netherlands Fellowship Programme (NUFFIC) to study for her Masters Degree with Wageningen University in the Netherlands. Here, she joined the Plant Sciences Department and specialized in Green-house Horticulture. Her Masters thesis was on the effect of storage temperature on quality attributes of two tomato varieties.

In March 2014, Praxedis was again awarded a Scholarship with NUFFIC NFP PhD Programme to study for her PhD degree on a sandwich program with Wageningen University at the Agricultural Economics and Rural Policy Group in the Netherlands. During the course of her $\mathrm{PhD}$, Praxedis followed various courses and has promoted the consumption of traditional leafy vegetables by educating people around Africa through article publications in Zimbabwean newspapers and a research presentation in African Association Agricultural Economist $\left(5^{\text {th }}\right)$, Addis Ababa, Ethiopia. This PhD thesis is an accomplishment of her study.

praxedisdube@gmail.com/praxedisdube@yahoo.com 


\section{Education Certificate}

\section{Praxedis Dube}

Wageningen School of Social Sciences (WASS)

\section{Completed Training and Supervision Plan}

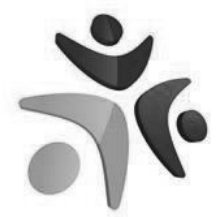

Wageningen School of Social Sciences
Name of the learning activity

\section{A) Project related competences}

WASS Introduction Course

$\mathrm{PhD}$ research proposal writing

Reviewing a Scientific Paper

Scientific Publishing

High impact writing in Science

'Food security In Zimbabwe: Demand for African indigenous vegetables

Economics and Sociology of Consumers and Household (ECH-303006)

B) General research related competences

'Food security In Zimbabwe: Demand for African indigenous vegetables'

Organizing session: The Economic Potential of African Indigenous Vegetables (AIVs) for African Food Markets, Addis Ababa

The economic potential of African indigenous vegetables (AIVS) for African food markets'

'Zim's traditional leafy veggie treasure'

'Lost crops: Food for thought for Zimbabwe'

'Traditional leafy vegetables into your garden'

C) Career related competences/personal development

Information on literacy Including EndNote Introduction (ILP)

Qualitative Data Analysis

Food Value Chain Research: Understanding Inter-Organizational Relationships

Qualitative Data Analysis with Atlas.it- a hands on practical

Research Methodology: From Topic to Proposal

Competence Assessment

Exploring Teaching Outside Academia

WASS PhD Education Programme

Last Stretch of the PhD Programme

Propositions workshop

Total
Department/Institute

Year ECTS

$\begin{array}{lcc}\text { Wageningen School of Social Sciences (WASS) } & 2014 & 1 \\ \text { Wageningen University (WUR) } & 2014 & 6 \\ \text { Wageningen Graduate Schools (WGS) } & 2014 & 0.1 \\ \text { WGS } & 2014 & 0.3 \\ \text { WGS } & 2017 & 1.3 \\ \text { WASS PhD-day } & 2017 & 1 \\ & & \\ \text { WUR } & 2014 & 6\end{array}$

African Association of Agricultural Economists: Transforming Smallholder Agriculture in Africa: The $2016 \quad 1$ Role of Policy and Governance, Addis Ababa, Ethiopia

African Association of Agricultural Economists: Transforming Smallholder Agriculture in Africa: The Role of Policy and Governance, Addis Ababa, Ethiopia

African Association of Agricultural Economists: Transforming Smallholder Agriculture in Africa: The $2016 \quad 1$ Role of Policy and Governance, Addis Ababa, Ethiopia

Newspaper article, http://www.sundaymail.co.zw/zims-traditional-leafy-veggie-treasure

Newspaper article, http://bulawayo24.com/index-id-news-sc-agriculture-byo-114547.html $\quad 2017 \quad 1$

$2017 \quad 1$

Newspaper article, http://bulawayo24.com/lifestyle/eats/120053

WGS

WASS

WASS

$2014 \quad 0.6$

$2017 \quad 2.5$

$2017 \quad 1.5$

WASS

$2014 \quad 1$

$2014 \quad 4$

$2014 \quad 0.3$

$2017 \quad 4$

$2017 \quad 0$

$2017 \quad 0$

$2017 \quad 0$

35.6

*One credit according to ECTS is on average equivalent to 28 hours of study load 


\section{Annex Questionnaire for Chapter 4}

\section{Annex 1}

\section{RESPONDENT NUMBER}

\section{QUESTIONNAIRES FOR PURCHASING AND EATING HABITS OF TLVs IN HOUSEHOLDS}

I am a PhD student with Wageningen Social Sciences (WASS), Chair Group Agricultural Economics and Rural Policy (AEP), the Netherlands. I am conducting a research on the Supply Chain of traditional leafy vegetables (TLVs) in several districts. In this questionnaire, I am particularly interested in the purchasing and eating habits of TLVs in your households. An essential component of my scientific research is finding out if the TLVs can improve food security in your region. For this purpose, I have developed this questionnaire and would kindly ask you to cooperate in answering questions. I would be grateful for your support and for your participation in the survey.

The information I would like to gather from you is going to be used for academic purposes only. I am not interested in your identity. It will remain confidential and may be published in scientific journals or presented at professional meetings, but only group patterns would be described and your identity would not be revealed. The question usually takes about 40 to 60 minutes.

\section{TO BE FILLED BEFORE THE INTERVIEW}

Name of the enumerator

Location/Area

Location/District

Date of interview

Start time of interview

End time of interview

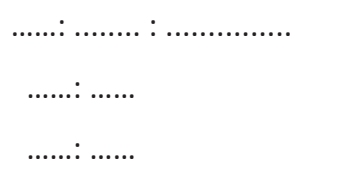




\section{SECTION 1 Socio-demographic data}

\subsection{Respondent information}

1. Are you living in a female headed/ male headed household

2. What is your relationship to the head of household

1.1 Relationship of respondent to the head of the household, Adopted from the Income, consumption and Expenditure survey questionnaire, 2001 (Central Statistical Office), Choose one

\begin{tabular}{|rl|l|}
\hline 1. & Head & \\
\hline 2. & Spouse & \\
\hline 3. & Son/daughter & \\
\hline 4. & Brother/sister & \\
\hline 5. & Nephew/niece/cousin & \\
\hline 6. & Grand child & \\
\hline 7. & Parent & \\
\hline 8. & Other relative (Specify) & \\
\hline 9. & Not related & \\
\hline
\end{tabular}

3. What is your marital status Married--Single--Widow/widower--Separated--Divorced

4. How old are you years

5. What is the gender of the respondent Male/Female

6. Have you ever attended school Yes/No 
7. What is your highest level and grade of education completed (1.2, Education completed categories, Choose one)

1.2 Education completed categories, Adopted from the Census 2012, ZimStat

\begin{tabular}{|l|l|}
\hline Level $9=9$ (Not known) & \\
\hline Level $8=8$ (None completed) & \\
\hline Level $0=1-3$ (Early education) & \\
\hline Level $1=1-7$ (Primary) & \\
\hline Level $2=1-6$ (Secondary) & \\
\hline Level 3 $=$ (Tertiary) & \\
\hline 1 Certificate/Diploma after primary & \\
\hline 2 Certificate/Diploma after secondary & \\
\hline 3 Graduate/Postgraduate & \\
\hline
\end{tabular}


8. What is your current occupation (1.3, Choose one)

1.3, Occupational classification (grouped), Adopted from the Census 2012, ZimStat

\begin{tabular}{|c|c|}
\hline & Tick one \\
\hline \multicolumn{2}{|c|}{ Government and Senior officials } \\
\hline \multicolumn{2}{|c|}{ Directors/ Managers and Company Secretaries } \\
\hline \multicolumn{2}{|l|}{ Natural Sciences } \\
\hline \multicolumn{2}{|l|}{ Business and Finance } \\
\hline \multicolumn{2}{|l|}{ Engineers and Technicians } \\
\hline \multicolumn{2}{|l|}{ Education } \\
\hline \multicolumn{2}{|l|}{ Health } \\
\hline \multicolumn{2}{|l|}{ Law and Security } \\
\hline \multicolumn{2}{|l|}{ Information } \\
\hline \multicolumn{2}{|l|}{ Social Sciences } \\
\hline \multicolumn{2}{|l|}{ Artists } \\
\hline \multicolumn{2}{|l|}{ Religion } \\
\hline \multicolumn{2}{|l|}{ Machine operators } \\
\hline \multicolumn{2}{|l|}{ Administration } \\
\hline \multicolumn{2}{|l|}{ Clerks and Secretaries } \\
\hline \multicolumn{2}{|l|}{ Transport } \\
\hline \multicolumn{2}{|l|}{ Services } \\
\hline \multicolumn{2}{|l|}{ Agriculture } \\
\hline \multicolumn{2}{|l|}{ Mining and Construction } \\
\hline \multicolumn{2}{|l|}{ Mechanics } \\
\hline \multicolumn{2}{|l|}{ Manufacturing } \\
\hline Others (SPECIFY) & \\
\hline
\end{tabular}

9. What is the name of your province of birth

10. What is the name of the place where you grew up

11. In which province does your household currently live

12. For how long have been staying in this place years

13. In which province do you mostly earn your living

14. What is your religion(1.4, Choose one) 
1.4, Religion classification

\begin{tabular}{|l|l|l|l|l|l|l|l|l|}
\hline Traditional & Roman catholic & Protestant & Pentecostal & Apostolic Sect & Other Christian & Muslim & None & Other (specify) \\
\hline & & & & & & & & \\
\hline
\end{tabular}




\subsection{Household information}

15. Is the head of the household you live in ----Female/ Male?

16. What is the occupation of the head of your household (1.5, Choose one)

1.5, Occupational classification (grouped) Adopted from the Census 2012, ZimStat

\begin{tabular}{|l|l|}
\hline & \multicolumn{1}{|c|}{ Tick one } \\
\hline Government and Senior officials & \\
\hline Directors/ Managers and Company Secretaries & \\
\hline Natural Sciences & \\
\hline Business and Finance & \\
\hline Engineers and Technicians & \\
\hline Education & \\
\hline Health & \\
\hline Law and Security & \\
\hline Information & \\
\hline Social Sciences & \\
\hline Artists & \\
\hline Religion & \\
\hline Machine operators & \\
\hline Administration & \\
\hline Clerks and Secretaries & \\
\hline Transport & \\
\hline Services & \\
\hline Agriculture & \\
\hline Mining and Construction & \\
\hline Mechanics & \\
\hline Manufacturing & \\
\hline Others (SPECIFY) & \\
\hline
\end{tabular}

17. What is the marital status of the head of the household Married--Single-Widow/widower--Separated-Divorced

18. How many people live in your household in each of the following age category (1.6, write the number)

1.6, Composition of people leaving in a household by age group and sex, Adopted from the Census 2012, ZimStat 


\begin{tabular}{|l|l|l|}
\hline Age group & Males & Females \\
\hline $0-14$ years & & \\
\hline $15-49$ years & & \\
\hline $50-64$ years & & \\
\hline 65 years ${ }^{+}$ & & \\
\hline Total & & \\
\hline
\end{tabular}

19. What is the average total monthly household income(1.7, Tick right answer)

1.7 Household monthly income range in US\$, Adopted from ZIMSTAT, Poverty Income Consumption and Expenditure Survey 2011/12 Report

\begin{tabular}{|l|l|}
\hline Income range in US\$ & Average total monthly household income (Tick) \\
\hline Less than 100 & \\
\hline $100-199$ & \\
\hline $200-350$ & \\
\hline $351-450$ & \\
\hline $451-495$ & \\
\hline $495-600$ & \\
\hline $601-800$ & \\
\hline $801-1000$ & \\
\hline Over 1000 & \\
\hline
\end{tabular}


20. Which of the following assets does your household own (1.8, Tick if Yes, column 2)

21. How many of following assets does your household own $(1.8$, Write the quantity, column 3)

\subsection{Assets ownership}

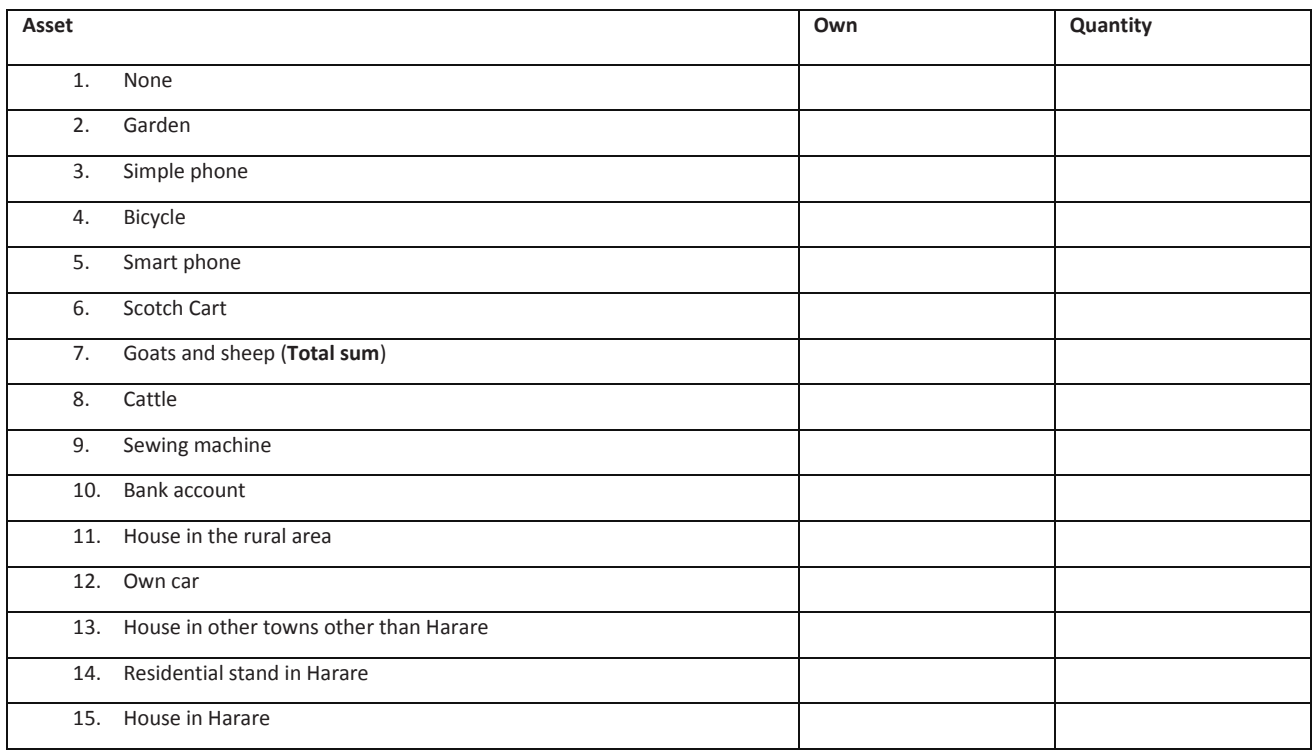

22. Who usually does the shopping of food in your household

23. Who usually decides on the household diet

24. Who usually decides on the type of vegetables to eat 
25. If your household could afford to spend more income on food, how would this influence your purchases for TLVs?

\begin{tabular}{|l|l|l|l|l|}
\hline Strong decrease & Decrease & unchanged & increase & Strong increase \\
\hline & & & & \\
\hline
\end{tabular}

26. IF DECREASE OR STRONG DECREASE (otherwise skip): On what would your household spend this extra income? 


\section{SECTION 2: Ethnic background}

(Write correct corresponding number on each of the ethnic question, specify other when relevant)

\begin{tabular}{|l|l|l|l|l|l|l|}
\hline Zezuru 1 & Manyika 2 & Karanga 3 & Ndebele 4 & Korekore 5 & Tonga 6 & Other......... \\
\hline
\end{tabular}

27. Your ethnic group

28. Your spouse's ethnic group

29. What is the ethnic group of the majority of population living in the region where you usually live

30. The ethnic of the father of the person who decides in your household about the diet

31. The ethnic of the mother of the person who decides in your household about the diet

32. Does your spouse prefer different AIVs than you Yes/No if Yes go to the next question, if No skip

33. Why is this so? (Choose one)

\begin{tabular}{|l|l|}
\hline & Tick \\
\hline Does not know them & \\
\hline Does not like the taste (Provide a name) & \\
\hline Lack of knowledge on how to prepare the ones not familiar with & \\
\hline Prefers the ones common in the area & \\
\hline Other (SPECIFY) & \\
\hline
\end{tabular}




\section{Section 3: TLV purchasing habits}

34. With which of the following TLVs are you familiar? (Third column of 3.1, tick)

35. Which of these TLVs does your household (hh) NOT like (Fourth column of 3.1, tick)

36. Which of these TLVs does your hh usually eat (Sixth column of 3.1, tick

37. Which of these TLVs does your hh usually buy (Fifth column of 3.1, tick)

38. Which of these TLVs does your hh usually grow (Seventh column of 3.1, tick)

39. Which of these TLVs does your hh prefer to eat (Eighth column of 3.1, tick) 


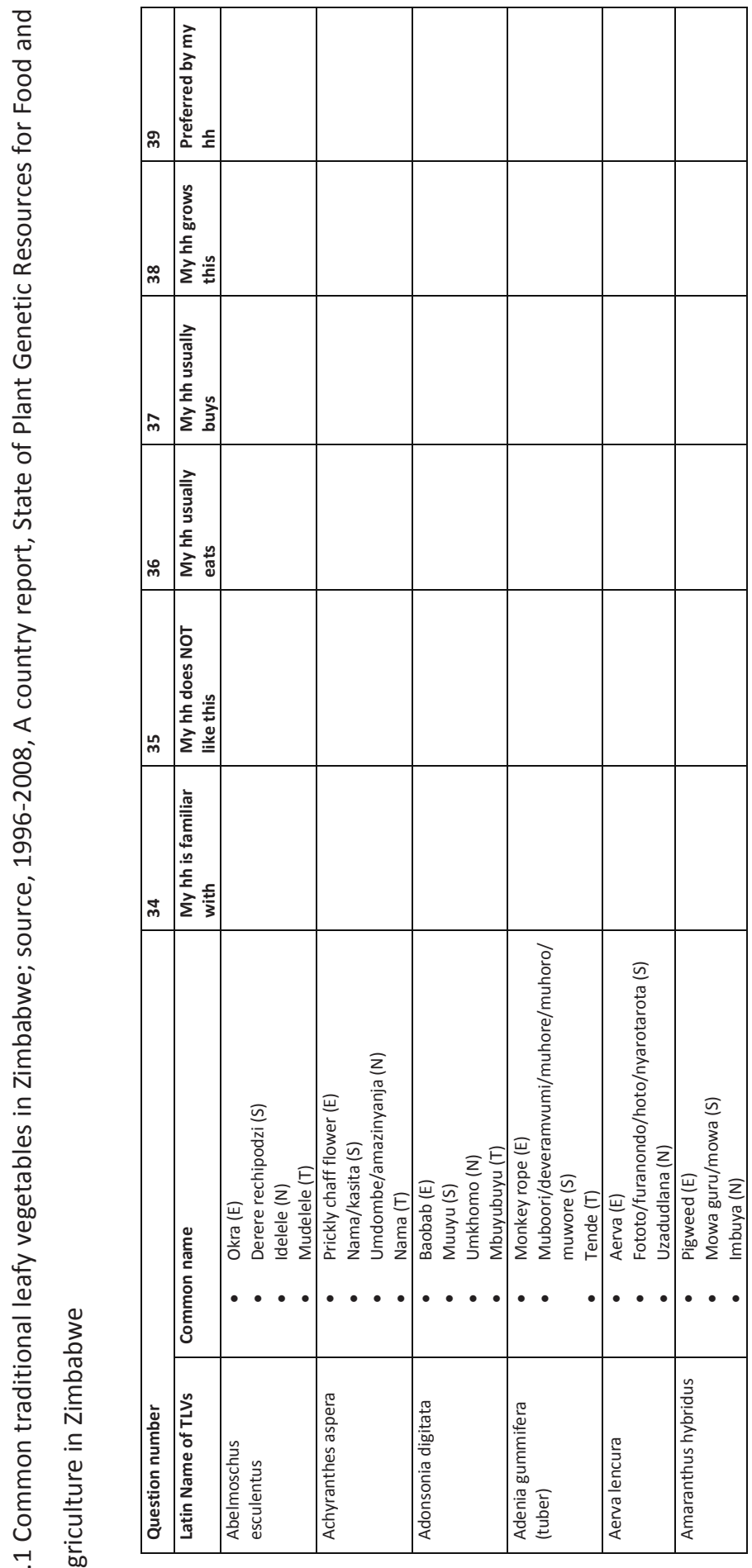




\begin{tabular}{|c|c|c|c|c|c|c|c|}
\hline \% & 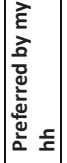 & & & & & & \\
\hline$\stackrel{\infty}{\infty}$ & 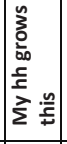 & & & & & & \\
\hline 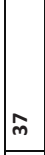 & 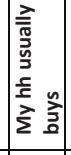 & & & & & & \\
\hline$\stackrel{\infty}{\infty}$ & 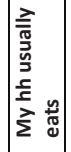 & & & & & & \\
\hline$\stackrel{m}{m}$ & 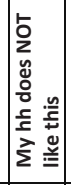 & & & & & & \\
\hline I & 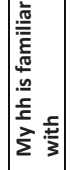 & & & & & & \\
\hline$\underset{8}{8}$ & 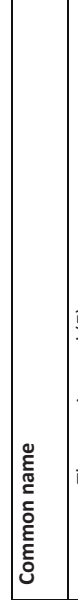 & 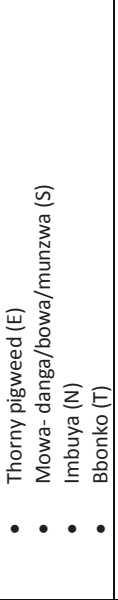 & 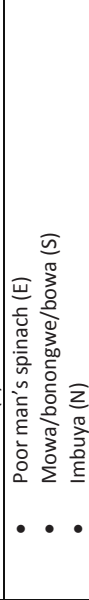 & 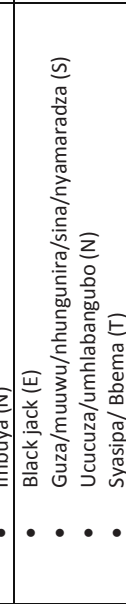 & 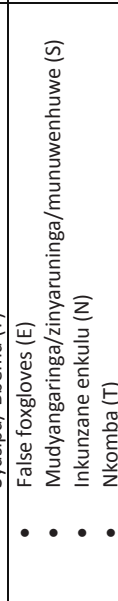 & 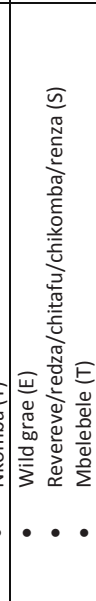 & 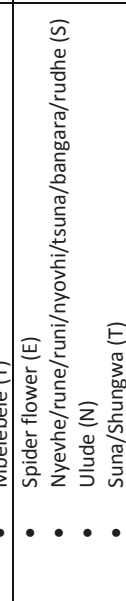 \\
\hline 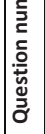 & 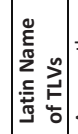 & 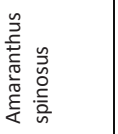 & 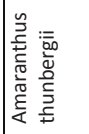 & 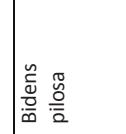 & 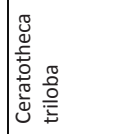 & 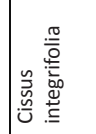 & 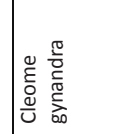 \\
\hline
\end{tabular}




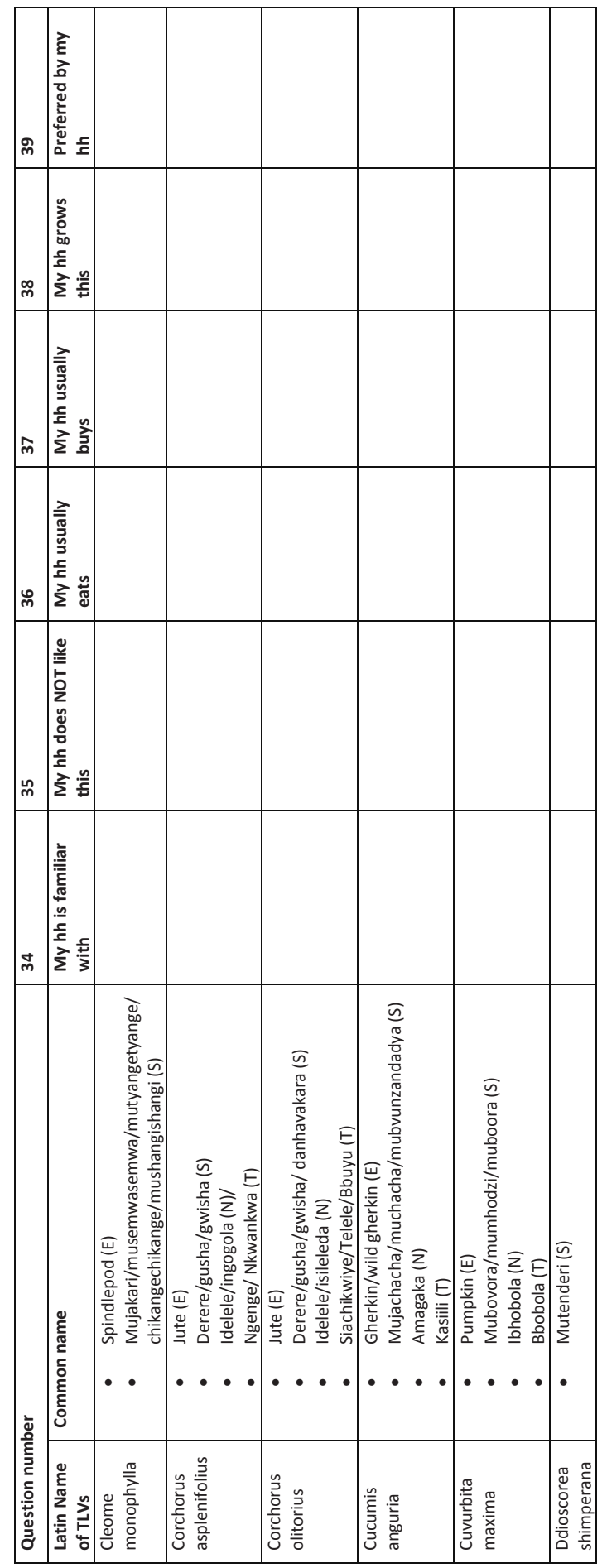




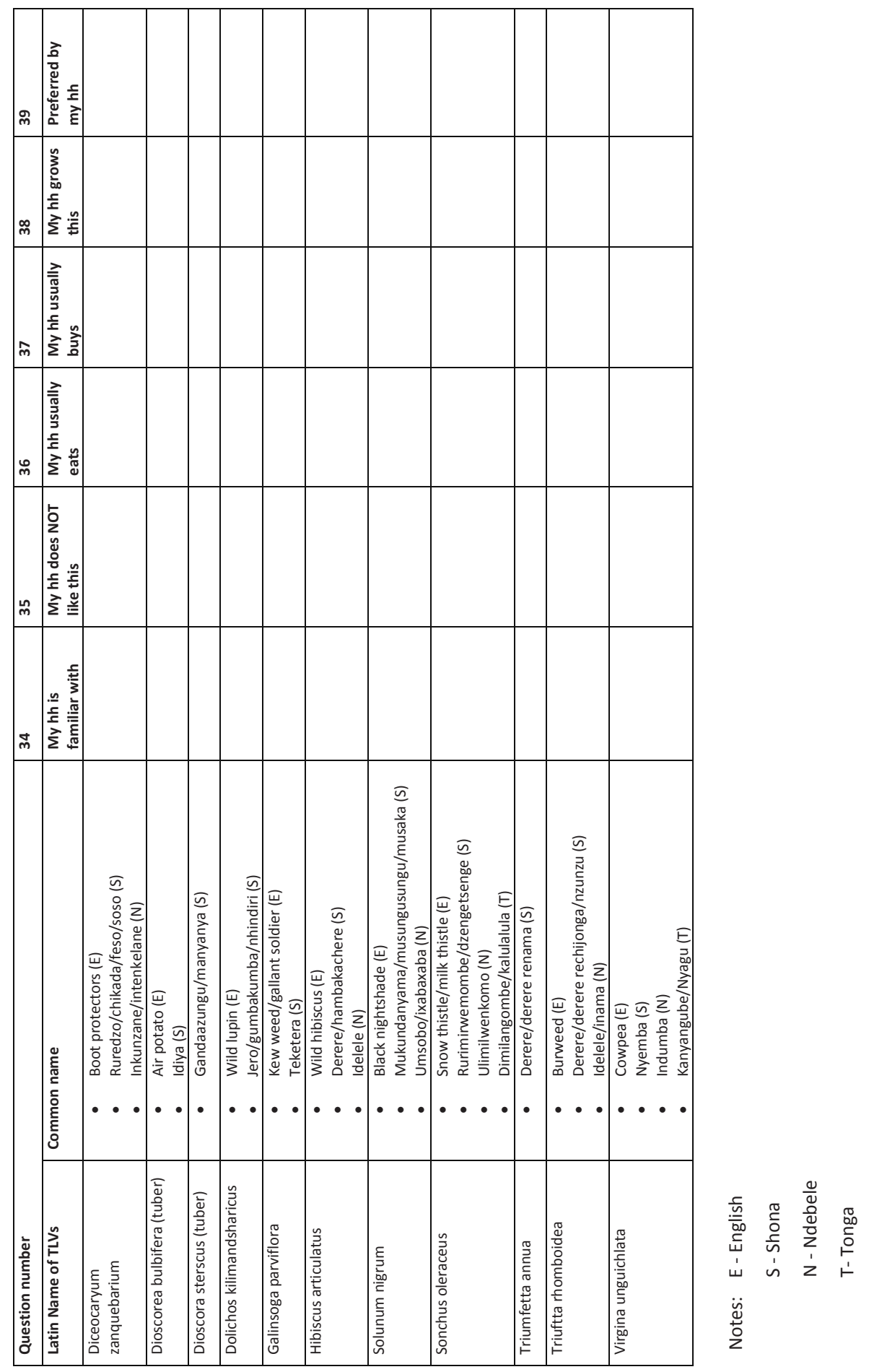


40. In which market place does your household usually obtain TLVs (Second column of

\section{2 ,you may choose more than one)}

41. Which of the markets does your household prefer (Third column of 3.2, choose the most preferred one)

\subsection{Market channels to obtain TLVs}

\begin{tabular}{|l|l|l|}
\hline Market place & Usually obtain & Preferred place \\
\hline Mbare-Musika Farmers Market & & \\
\hline Mbare-Musika retail- stalls & & \\
\hline Central Business District - stalls & & \\
\hline Streets & & \\
\hline Supermarkets & & \\
\hline Road side market & & \\
\hline Farmers markets & & \\
\hline Door to Door & & \\
\hline My hh does not buy but grows in own garden/field & & \\
\hline Buy from neighbour's garden & & \\
\hline My hh does not buy but gets it from neighbour's garden for free & & \\
\hline My hh does not buy but gets it from neighbour's garden in exchange for labour or other food & & \\
\hline My hh does not buy but collects from the forest- grows naturally in forest & & \\
\hline Other markets (SPECIFY) & & \\
\hline
\end{tabular}

42. What is the reasons for choosing the particular markets (3.3)

3.3 Reasons for choosing the particular markets

\begin{tabular}{|l|l|l|l|l|l|}
\hline & Very unimportant & Somewhat unimportant & Indifferent & Somewhat important & Very important \\
\hline Close & & & & & \\
\hline Cheap & & & & & \\
\hline High quality & & & & & \\
\hline Broad choice & & & & & \\
\hline Reliable & & & & & \\
\hline Credit arrangements & & & & & \\
\hline Bulky/many & & & & & \\
\hline Others (SPECIFY) & & & & & \\
\hline
\end{tabular}


43. What is the main reason of your household purchasing TLVs, (3.4)

\subsection{Main reason for purchasing TLVs}

My household usually purchases TLVs because;

\begin{tabular}{|l|l|l|l|l|l|}
\hline & $\begin{array}{l}\text { Completely } \\
\text { disagree }\end{array}$ & $\begin{array}{l}\text { Somewhat } \\
\text { disagree }\end{array}$ & Indifferent & $\begin{array}{l}\text { Somewhat } \\
\text { agree }\end{array}$ & $\begin{array}{l}\text { Completely } \\
\text { agree }\end{array}$ \\
\hline they are cheap & & & & & \\
\hline they are locally produced & & & & & \\
\hline I like their taste & & & & & \\
\hline They improve the health of my family & & & & & \\
\hline I prefer them to exotic vegetables & & & & & \\
\hline they are easy to prepare & & & & & \\
\hline my family considers them traditionally & & & & & \\
\hline they are sold everywhere & & & & & \\
\hline they are readily available & & & & & \\
\hline they have medical properties & & & & & \\
\hline they have beneficial properties & & & & & \\
\hline they are nutritious & & & & & \\
\hline they are high quality food & & & & & \\
\hline $\begin{array}{l}\text { they reminds us of the old days when we } \\
\text { were in rural areas }\end{array}$ & & & & & \\
\hline only vegetables that can grow in my area & & & & & \\
\hline Only vegetables available on the market & & & & & \\
\hline Other (SPECIFY) & & & & \\
\hline
\end{tabular}


44. Under which conditions would your household purchase more TLVs, 3.6, (Tick)

\subsection{Conditions for purchasing more TLVs}

\begin{tabular}{|l|l|l|l|l|l|}
\hline Conditions of purchasing more TLVs & $\begin{array}{l}\text { Completely } \\
\text { disagree }\end{array}$ & $\begin{array}{l}\text { Somewhat } \\
\text { disagree }\end{array}$ & Indifferent & $\begin{array}{l}\text { Somewhat } \\
\text { agree }\end{array}$ & $\begin{array}{l}\text { Completely } \\
\text { agree }\end{array}$ \\
\hline $\begin{array}{l}\text { If they were freshly harvested from neighbour's } \\
\text { garden }\end{array}$ & & & & & \\
\hline If they were fresher & & & & & \\
\hline If they were more tender, without flowers & & & & & \\
\hline If they were produced under organic agriculture & & & & & \\
\hline If my hh would be aware of their nutritional value & & & & & \\
\hline $\begin{array}{l}\text { If my hh would be advised to consume them for } \\
\text { health reasons }\end{array}$ & & & & & \\
\hline $\begin{array}{l}\text { If my hh cannot afford meat } \\
\text { If they were the only available vegetables on the } \\
\text { market }\end{array}$ & & & & & \\
\hline $\begin{array}{l}\text { If they were of better quality (e.g. a mixed } \\
\text { bundles has green and purple stemmed bunched } \\
\text { together) }\end{array}$ & & & & & \\
\hline $\begin{array}{l}\text { If they would be the vegetables which could only } \\
\text { be grown in my area }\end{array}$ & & & & & \\
\hline $\begin{array}{l}\text { If my hh would like them more } \\
\text { If they were more nicely prepared for sale }\end{array}$ & & & & & \\
\hline If they were more nicely packaged & & & & & \\
\hline
\end{tabular}


45. Please rate the following characteristics to show their importance to your household for buying food, 3.7, (Tick)

3.7 The most important food characteristic is its

\begin{tabular}{|l|l|l|l|l|l|}
\hline Factors & $\begin{array}{l}\text { Completely } \\
\text { disagree }\end{array}$ & $\begin{array}{l}\text { Somewhat } \\
\text { disagree }\end{array}$ & Indifferent & $\begin{array}{l}\text { Somewhat } \\
\text { agree }\end{array}$ & $\begin{array}{l}\text { Completely } \\
\text { agree }\end{array}$ \\
\hline Price & & & & & \\
\hline Freshness/look & & & & & \\
\hline Taste & & & & & \\
\hline Nutrition & & & & & \\
\hline Health & & & & & \\
\hline Safety & & & & & \\
\hline $\begin{array}{l}\text { Knowledge on how to prepare } \\
\text { them }\end{array}$ & & & & & \\
\hline Time of preparing them & & & & & \\
\hline
\end{tabular}

46. On average how much does your household spend on TLVs per week during off season US\$

47. On average how much does your household spend on TLVs per week during on season US\$

48. If your hh would have enough income and if the quality of the TLVs offered in your preferred market place would completely match your expectations, how much would your household like to spend on TLVs per week US\$

49. In your opinion, did the availability of TLVs in the markets change since the melting down of the economy of Zimbabwe (2008) (3.9, Choose one)

3.9 Availability of TLVs

\begin{tabular}{|l|l|l|l|l|l|}
\hline Season & strongly decrease & decrease & No difference & Increase & strongly increase \\
\hline Off Season & & & & & \\
\hline On Season & & & & & \\
\hline
\end{tabular}


50. During off-season when fresh TLVs are unavailable, what substitutes does your household prefer most (Table 3.10)

3.10 Household substitution

\begin{tabular}{|l|l|l|l|l|l|}
\hline & Never & Sometimes & Regularly & Frequently & Very frequently \\
\hline Dried TLVs & & & & & \\
\hline Boiled and dried TLVs & & & & & \\
\hline Rape & & & & & \\
\hline Covo & & & & & \\
\hline Tsunga & & & & & \\
\hline Cabbage & & & & & \\
\hline Other (SPECIFY) & & & & & \\
\hline
\end{tabular}

51. How far from your living place is the market place located that you prefer visiting for purchasing TLVs? $\mathrm{km}$

52. How much time approximately takes it to travel from your living place to the preferred market place? minutes (one way)

53. How far from your living place is the closest TLV market place located? $\mathrm{km}$

54. How often does your household eat TLVs (4.2, Choose one only in each column)

4.2 Eating rates

\begin{tabular}{|l|l|l|}
\hline & Off season & On season \\
\hline Less than once per month & & \\
\hline At most 2 times per month & & \\
\hline At most once per week (=At most 4 times per month) & & \\
\hline Every second day & & \\
\hline Once every day & & \\
\hline Twice every day & & \\
\hline Three times every day & & \\
\hline
\end{tabular}


55. IF less than once per month (otherwise skip): What are the reasons of your household not eating TLVs (3.5)

\section{5, Main reason for not eating TLVs}

My household does not eat TLVs because;

\begin{tabular}{|c|c|c|c|c|c|}
\hline & $\begin{array}{l}\text { Completely } \\
\text { disagree }\end{array}$ & $\begin{array}{l}\text { Somewhat } \\
\text { disagree }\end{array}$ & Indifferent & $\begin{array}{l}\text { Somewhat } \\
\text { agree }\end{array}$ & $\begin{array}{l}\text { Completely } \\
\text { agree }\end{array}$ \\
\hline $\begin{array}{l}\text { they are not available in the local } \\
\text { markets }\end{array}$ & & & & & \\
\hline doesn't like the taste & & & & & \\
\hline doesn't know them & & & & & \\
\hline $\begin{array}{l}\text { not interested, felt it's for poor } \\
\text { people }\end{array}$ & & & & & \\
\hline $\begin{array}{l}\text { not interested because is of poor } \\
\text { quality }\end{array}$ & & & & & \\
\hline doesn't know where to get them & & & & & \\
\hline doesn't know how to prepare them & & & & & \\
\hline lacks time to prepare them & & & & & \\
\hline prefers exotic vegetables & & & & & \\
\hline Other (SPECIFY) & & & & & \\
\hline
\end{tabular}

56. Are TLVs mainly served as main dish or side dish in your household?

\begin{tabular}{|l|l|l|}
\hline & Off season & On season \\
\hline Mainly served as main dish & & \\
\hline Mainly served as side dish & & \\
\hline Other (SPECIFY) & & \\
\hline
\end{tabular}


57. How important does your household regard eating of TLVs in the daily diet? (4.3, second row, Choose one only)

58. Do you think the eating of TLVs is important for all age groups in your household? (4.3, row 3 to 6 , Choose one only)

4.3 TLVs importance in the daily diet and on stated age groups

\begin{tabular}{|l|l|l|l|l|l|}
\hline & $\begin{array}{l}\text { very } \\
\text { unimportant }\end{array}$ & $\begin{array}{l}\text { Somewhat } \\
\text { unimportant }\end{array}$ & $\begin{array}{l}\text { Neither important nor } \\
\text { unimportant }\end{array}$ & $\begin{array}{l}\text { Somewhat } \\
\text { important }\end{array}$ & $\begin{array}{l}\text { very } \\
\text { important }\end{array}$ \\
\hline Daily diet & & & & & \\
\hline $0-17$ years & & & & & \\
\hline $\begin{array}{l}18-65 \\
\text { years }\end{array}$ & & & & & \\
\hline 65 years & & & & & \\
\hline
\end{tabular}

59. How do you rate the eating of TLVs in your household since the melting down of the economy of Zimbabwe (2008)? (4.4, Choose one only)

4.4 Rating of the eating during the previous 7 years

\begin{tabular}{|l|l|l|l|l|l|}
\hline Season & strongly decrease & decrease & No difference & increase & strongly increase \\
\hline Off season & & & & & \\
\hline On season & & & & & \\
\hline
\end{tabular}

60. Can you estimate the quantity of TLVs eaten by your household per each time of eating? (a standard bundle-100grams, a standard cup-60grams) (4.5, Tick one only)

4.5 Estimates of the quantities of TLVs consumed per each time

\begin{tabular}{|l|l|l|l|l|l|}
\hline Quantities & 1 & 2 & 3 & 4 & 5 \\
\hline A standard bundle & & & & & \\
\hline A standard cup & & & & & \\
\hline
\end{tabular}

\section{Thank you}

